University of Louisville

ThinkIR: The University of Louisville's Institutional Repository

Electronic Theses and Dissertations

$12-2017$

\title{
All-optical switching in ferromagnetic superlattices.
}

Faren Hoveyda Marashi

University of Louisville

Follow this and additional works at: https://ir.library.louisville.edu/etd

Part of the Condensed Matter Physics Commons

\section{Recommended Citation}

Hoveyda Marashi, Faren, "All-optical switching in ferromagnetic superlattices." (2017). Electronic Theses and Dissertations. Paper 2833.

https://doi.org/10.18297/etd/2833

This Doctoral Dissertation is brought to you for free and open access by ThinkIR: The University of Louisville's Institutional Repository. It has been accepted for inclusion in Electronic Theses and Dissertations by an authorized administrator of ThinkIR: The University of Louisville's Institutional Repository. This title appears here courtesy of the author, who has retained all other copyrights. For more information, please contact thinkir@louisville.edu. 


\title{
ALL-OPTICAL SWITCHING IN FERROMAGNETIC SUPERLATTICES
}

\section{By}

Farzaneh Hoveyda Marashi

B.S., Shahid Beheshti University, 2007

M.S., University of Mysore, 2010

\author{
A Dissertation \\ Submitted to the Faculty of the \\ College of Arts and Sciences of the University of Louisville \\ in Partial Fulfillment of the Requirements \\ for the Degree of \\ Doctor of Philosophy \\ in Physics \\ Department of Physics and Astronomy \\ University of Louisville \\ Louisville, Kentucky
}

December 2017 
Copyright 2017 by Farzaneh Hoveyda Marashi

All rights reserved 



\title{
ALL-OPTICAL SWITCHING IN FERROMAGNETIC SUPERLATTICES
}

\author{
By
}

Farzaneh Hoveyda Marashi

B.S., Shahid Beheshti University, 2007

M.S., University of Mysore, 2010

A Dissertation Approved on

November 29, 2017

by the following Dissertation Committee:

Dr. Serban Smadici

Dr. Chakram S. Jayanthi

Dr. Gamini Sumanasekera

Dr. Robert Cohn 


\section{ACKNOWLEDGMENTS}

First and foremost, I would like to express my sincere gratitude to my advisor Dr. Smadici for giving me the opportunity to work on a meaningful project. I appreciate his dedication to make my experience productive. He has been very generous with his time and knowledge to help me with different steps from experiments to preparation for attending a conference. Beyond all, I have always appreciated his willingness to listen to my viewpoints, without presumptions. This certainly helped me to develop invaluable interpersonal skills.

I am indebted to my committee members Dr. Jayanthi, Dr. Gamini Sumanasekera and Dr. Cohn for their support and accessibility through these years. Thank you for the interesting and insightful questions during my defense session.

Dr. Jayanthi, Thank you for providing an empowering inspiration. I have always appreciated having a successful woman physicist as the department chair. Moreover, I am very grateful for your work around funding issues to support Meysam and me. It has also been heartening to see you in the department, on the occasions that I worked overnight.

It is my pleasure to thank Dr. Gamini for supporting me from the very beginning, both financially and academically. I also appreciated his sense of responsibility when I was his TA.

I would also like to thank Dr. Davis, graduate advisor of the physics department, for supporting me over these years, for his advice on many occasions, and for making us feel we can reach him in the wake of any problem. I appreciate that he always ensured that we are aware of deadlines and procedures. In addition, it was heartwarming to see the postcards, his wife sent us, International students, as the political climate was getting tough.

I am pleased to express my appreciation to Mary Gayle and Rea Diehlmann for the usual prompt help and for creating a welcoming atmosphere in the physics office. I 
am also grateful to Joshua Rimmer for making the parts we needed even if it was right before Christmas.

Over these years, I have benefitted from the knowledge and expertise of friendly cleanroom staff. I am grateful to Dr. Julia Aebersold, cleanroom manager, for accommodating our needs. In addition, I appreciate Evgeniya Moiseeva, Xiaojin Wang, Curt McKenna, and Tereza Paronyan as well as Julia, for training me to use different equipment.

I have been lucky to have some of the nicest people as my lab mates and friends. In particular, Sahar Pishgar and Sahar Goharshenasan, thank you for your precious friendship. It was always delightful to see your faces in the department. Sahar P, thanks for being a caring, thoughtful and calm support. Sahar G, thanks for your kindness in helping me out on some of the most chaotic days. Erich Hohenstein, I appreciate your work on the magnetic simulations, even after moving back to your country. Victoria, thanks for generosity with your cute cat, I truly enjoyed her company during a tense time. Rodrigo Wiederkehr, I appreciate your time and patience in teaching me precise optical alignment. Behnaz and Behnoush, thanks for your kindness in taking care of our little pigies each time we left for APS meetings. I am also, grateful to Romaneh for her honest views and throwing memorable parties. Azadeh Davodi, thank you for keeping in touch and for your invaluable friendship. In addition, I am grateful to Han Xue, Scott Smith, Richard Judge, and Niknam Momenzadeh.

I owe my deepest gratitude to Meysam Akhtar. I have been fortunate to enjoy his company from college years up to present. He has also been a humble teacher, skilled in articulating his knowledge on different subjects including those I failed to learn in the class. Keeping up with him, demanded me to grow every day. Beyond all, I have been greatly inspired by his commitment to his core principles. He has certainly made my life experience more meaningful. Thank you for your love, humor, patience and dedication.

I would like to appreciate my uncle for keeping in touch. It is always uplifting to talk with you. I am also sincerely grateful to Mohamad and Firouze for their unconditional love. Thanks for inspiring me with your thoughts, creativity and talents. I have been immensely delighted with flowers and carefully chosen gifts you sent me over these years. Thank you for always being there for me. 
I would not be able to reach my goals without the constant encouragement and support of my parents, Enayat and Fakhri. I cannot appreciate them enough for their love and devotion. Thank you for always believing in me and supporting my decisions, no matter if they did not match your dreams. 


\section{ABSTRACT \\ ALL-OPTICAL SWITCHING IN FERROMAGNETIC SUPERLATTICES}

Farzaneh Hoveyda

November 29, 2017

The unexpected observation of ultrafast demagnetization (UDM) and subsequent time-resolved studies of laser-induced magnetization switching opened a new door to both fundamental physics and technological applications of magnetic materials. Alloptical switching (AOS) can be initiated faster than the precession limit, hinting to its potential in increasing the writing speed and data storage density. However, notwithstanding considerable research interest, the mechanism of AOS in ferromagnetic materials remained unclear.

Ferromagnetic superlattices were deposited on glass substrates with e-beam evaporation and sputtering. Magnetization curves were measured in magneto-optical and vibrating sample magnetometer experiments. A femtosecond Ti:S laser was utilized in a writing setup to induce $\mathrm{AOS}$ in $\mathrm{Co} / \mathrm{Pd}$ ferromagnetic superlattices at different fluences and beam polarizations. Magnetic force and polarizing microscopy were applied to image the magnetic structure and identify optimal AOS parameters.

High-repetition rate pulses of the Ti:S laser resulted in heat accumulation in the samples. For a better understanding of the relation between temperature and domain wall 
motion, we solved the heat diffusion equation numerically. The solution for our sample displayed a tilted thermal wave front, consistent with the tilted magnetic domains observed. This supports our model of AOS, in which thermal forces acting on domain walls leads to their expansion and magnetization switching (chapter 3).

Polarizing microscopy images also revealed a complementary pattern of magnetic domains after laser writing, suggesting that demagnetizing fields are not negligible. Furthermore, comparison of pump-probe UDM measurements with AOS writing measurements pointed to a demagnetized state before AOS emergence. This motivated us to apply micromagnetic simulations to investigate the time evolution of a demagnetized state and in particular, the role of demagnetizing fields in the development of different final states. We show using this method that demagnetizing fields can nucleate and, together with thermally induced forces, develop a switched state (chapter 4).

Using the pump-probe setup, we measured the frequency dependence of laserinduced temperature modulations in $\mathrm{Co} / \mathrm{Au}, \mathrm{Co} / \mathrm{Ag}$ and $\mathrm{Co} / \mathrm{Pd}$ superlattices on glass substrates. Green's function solutions of the heat diffusion equation show that a glass layer with properties distinct from the glass substrate is present near the metallic superlattices (chapter 5). 


\section{TABLE OF CONTENTS}

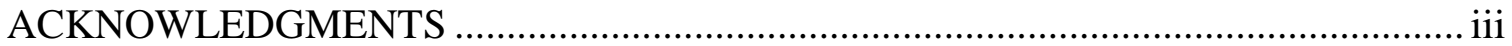

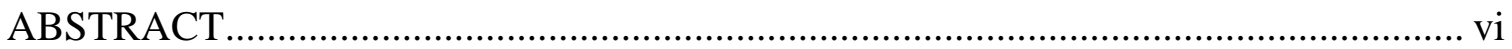

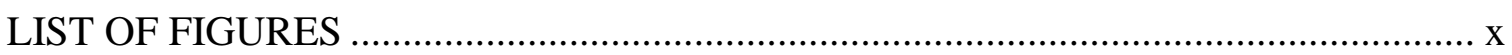

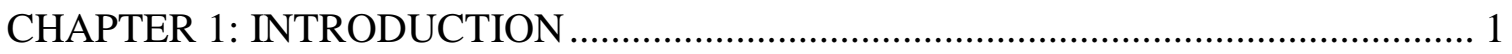

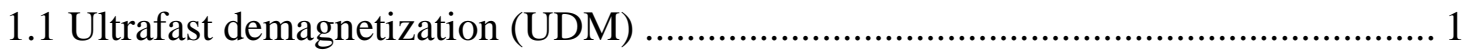

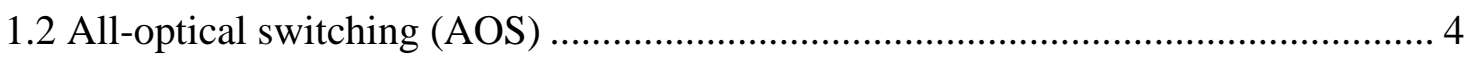

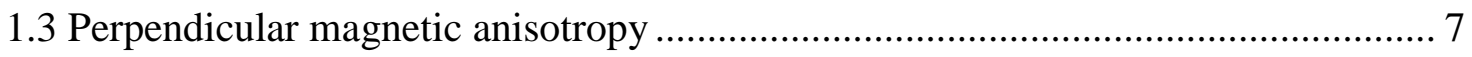

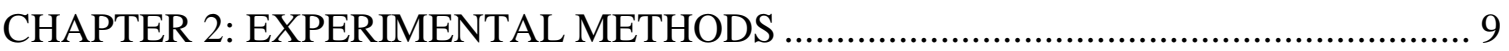

2.1 Sample preparation and thickness measurement.................................................. 9

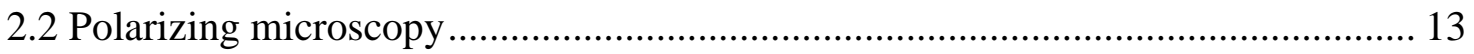

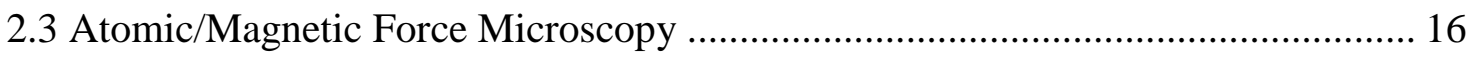

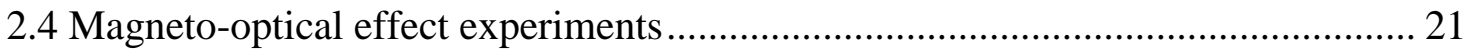

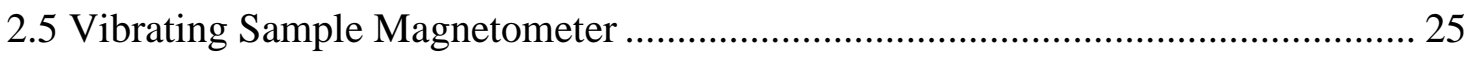

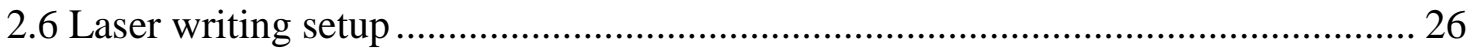

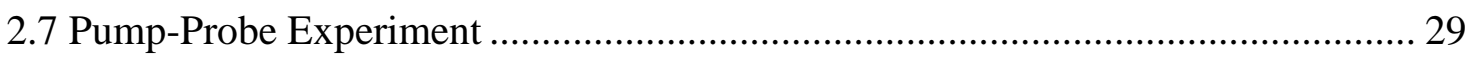

CHAPTER 3: HEAT ACCUMULATION AND ALL-OPTICAL SWITCHING BY DOMAIN WALL MOTION IN CO/PD SUPERLATTICES .......................................... 36

3.1 Introduction

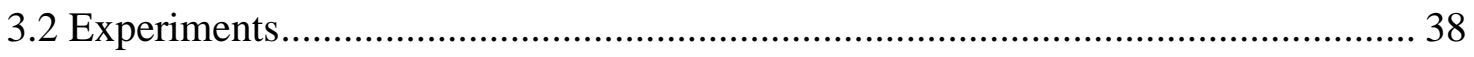

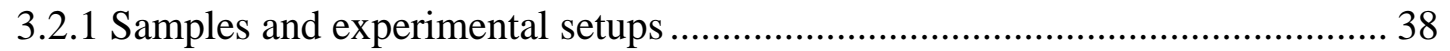

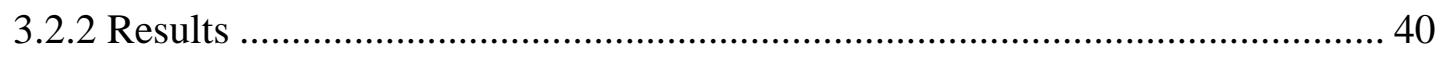

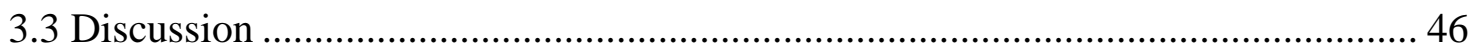

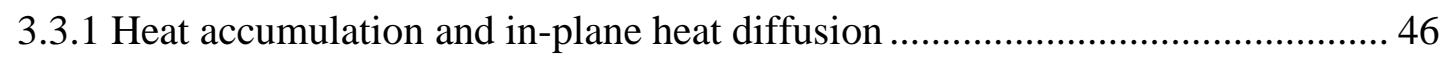

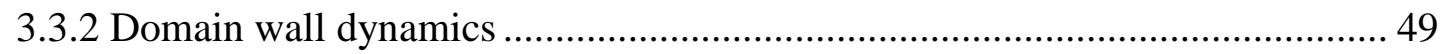

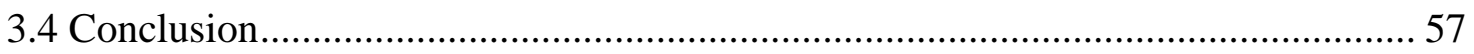

CHAPTER 4: DEMAGNETIZING FIELDS IN ALL-OPTICAL SWITCHING.............59

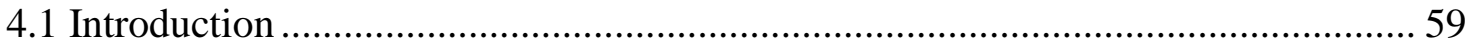

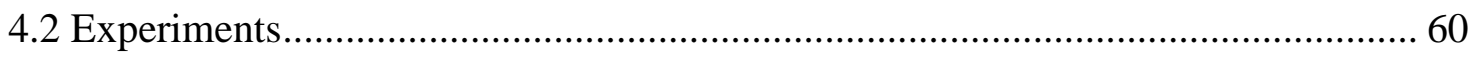

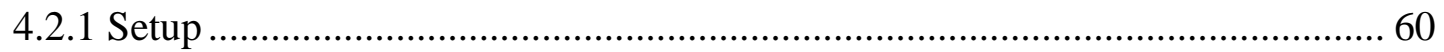

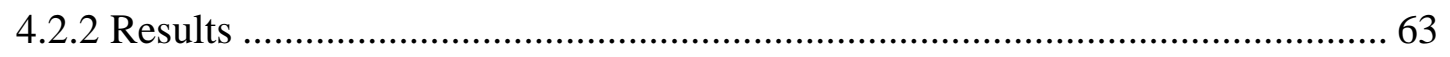

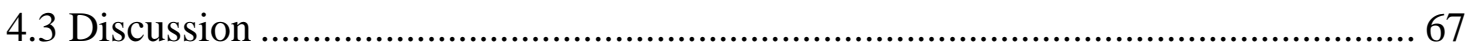

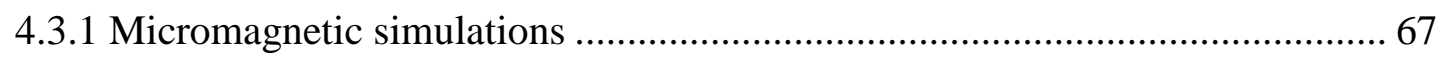

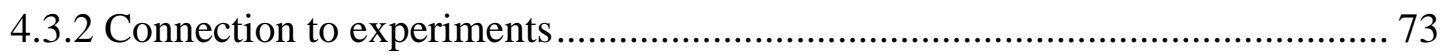

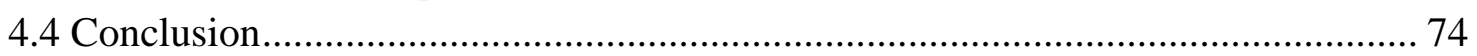




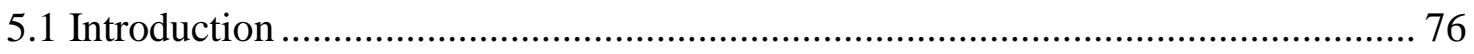

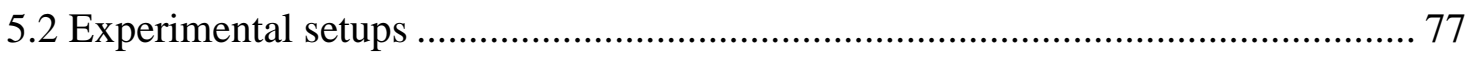

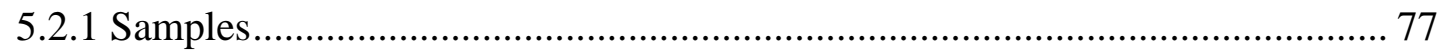

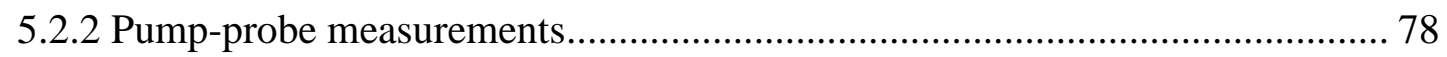

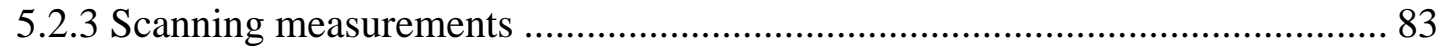

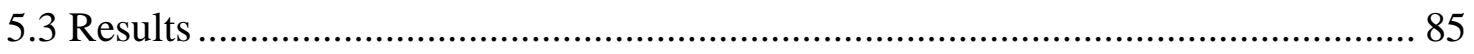

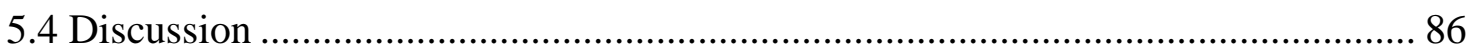

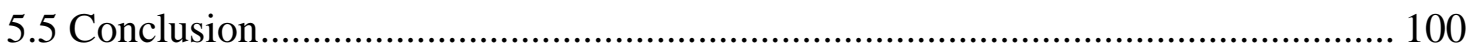

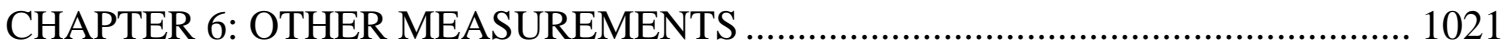

6.1 Temperature dependence of the coercive field in GdFeCo films..................... 1021

6.2 Detecting the magnetization reorientation with doping ................................. 1032

6.3 Finding the optimal thickness for PMA ....................................................... 1054

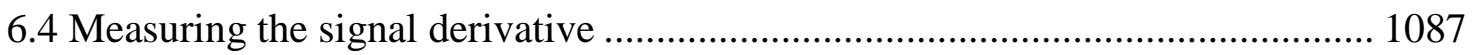

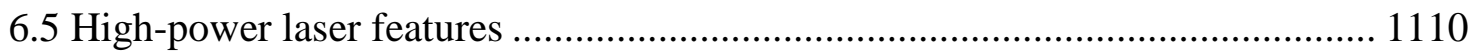

CHAPTER 7: SUMMARY AND OUTLOOK ….................................................... 115

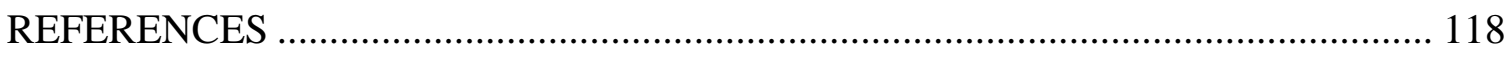

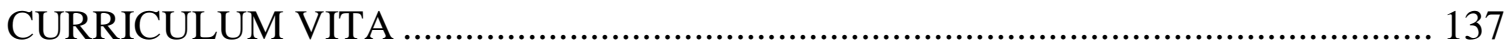




\section{LIST OF FIGURES}

Figure 1.1 | Timescale of magnetization precession. The plot shows polar MOKE results of a $\mathrm{Co} / \mathrm{Au}$ sample [3]. The $\mathrm{y}$-axis denotes polarization rotation and is proportional to the perpendicular component of magnetization perpendicular to the sample surface.

Figure 1.2 | First demonstration of ultrafast demagnetization in a Ni sample. The timedependence of remanent magnetization (the magnetization at zero applied field), measured with a pump-probe experiment, shows a sudden drop in magnetization within 260 fs of laser excitation [1]. The result was normalized to the signal obtained without the pump beam.

Figure 1.3 | The M3TM model describes UDM as the result of multiple spin-flip scatterings that occur during the temperature equilibration of electrons with the lattice [7]. Initial models suggested that UDM occurs due to the increase of temperature (since the magnetization reduces as the temperature increases toward Curie temperature). However, magnetization dynamics associated with transfer of angular momentum should be taken into account and the microscopic $3 \mathrm{~T}$ model indicated that UDM results from dissipation of electron's angular momentum into the lattice.

Figure 1.4 | Magneto-optical images of GFC sample (a) before and (b) after scanning under a pulsed laser beam. $\mathrm{L}, \sigma+$ and $\sigma$ - denote the linear polarization, righthanded and left-handed circularly polarization, respectively. Dark (bright) areas correspond to domains with down (up) magnetization [9].

Figure 1.5 | Time-dependence of magnetization during AOS in a GFC sample, measured with pump-probe microscopy. This result revealed that AOS in GFC develops on a $100 \mathrm{ps}$ timescale from pump pulse excitation [11]. The y-axis in the lower plot shows the polarization rotation, which is proportional to magnetization. The angle change from positive angle to negative confirms magnetization reversal.

Figure 1.6 | Time evolution of magnetization of individual sub-lattices. In the proposed AOS mechanism in ferrimagnet GFC, reversal emerges due to a transient ferromagnetic-like state, in which the magnetic moments of rare earth (RE) and transition-metal (TM) elements are parallel [12]....

Figure 1.7 | Polarizing microscopy images of AOS with a single pulse in a GFC sample with initial magnetization pointing up (bright background, top row) and down (dark

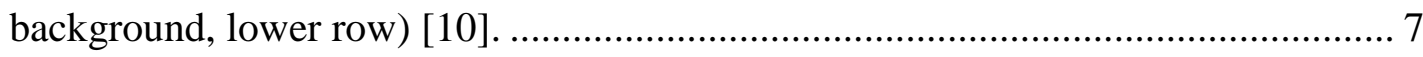

Figure 2.1 | Sketch of a typical magnetron sputtering. ............................................. 9 Figure 2.2 | Sketch of electron-beam vapor deposition system. Pellets of target materials are placed in crucibles at the bottom of the vacuum chamber. 10

Figure 2.3 | Sample holder used during deposition for depositing films with a variable thickness 
Figure 2.4 | The sample is attached to a motorized stage. A HeNe laser beam is shined on the sample and the intensity of transmission and reflection beams is measured as the stage move the sample along its length.......................................................... 12

Figure 2.5 The diagram shows the thickness variation along a GdFeCo (GFC) sample deposited with the method described in figure (2.3) ........................................... 12

Figure 2.6 | Polarizing microscopy in transmission mode. Depending on sample transparency, it can be used in transmission or reflection mode.

Figure 2.7 | Image of magnetic domains produced by subtracting images taken at analyzer angles \pm 1 degrees.

Figure 2.8 | Polarizing microscope images with analyzer angle at -1 (a) and +1 (b) with respect to extinction angle. Image (c) is obtained by subtracting (a) and (b).

Figure 2.9 | Distinguishing magnetic and optical birefringence. Sample was rotated and imaged at (a) $0^{\circ}$, (b) $45^{\circ}$, (c) $90^{\circ}$, (d) $135^{\circ}$. The contrast of magnetic domains is fixed, while the wings (arrows) contrast is reversed with angle: bright in $(a, b)$ and dark in (c, d)

Figure 2.10 | Atomic force microscope (AFM) works by detecting deflection of a cantilever due to forces between sample and the tip.

Figure 2.11 | Images of a $\mathrm{Co} / \mathrm{Pd}$ sample acquired by (a) polarizing microscope (b) MFM show the magnetic domains between stripes written with a Ti:S laser..... 17

Figure 2.12 | MFM imaging procedure to avoid topography features from contributing to the magnetic image: (a) AFM/MFM head scans the surface to obtain the topography (b) of the surface. (c) The topography data is then used as the reference (the head moves up and down according to topography) for rescanning and detecting the magnetic forces at a predefined height ' $h$ ' where other forces are negligible. (d) Magnetic image of a floppy disc (here $\mathrm{h}=50 \mathrm{~nm}$ ), acquired simultaneously with the topography image (b) of the surface. $\mathrm{SP}=0.690 \mathrm{~V}, \mathrm{G}=3.5$.

Figure 2.13 | simultaneously obtained AFM (left) and MFM (right) images of a floppy disc. $\mathrm{SP}=0.584 \mathrm{~V}, \mathrm{G}=3.5$

Figure 2.14 | Simultaneously obtained MFM (top) and AFM (bottom) images of a floppy disc. $\mathrm{SP}=0.776 \mathrm{~V}, \mathrm{G}=3.5$ 20

Figure 2.15 | Hysteresis loops of a thin Co film (15 nm) measured using MOKE (left) and Faraday (right) experiments.

Figure 2.16 | MOKE setup in longitudinal geometry. Longitudinal MOKE is used for measuring in-plane components of the magnetization... 22

Figure 2.17 | MOKE setup in polar geometry. Polar MOKE is sensitive to out-of-plane components of the magnetization.

Figure 2.18 | Sketch of vibrating sample magnetometer (VSM). Vibrating a sample in a magnetic field, leads to a change in the magnetic flux density. This induces an electric current in four pickup coils surrounding the sample holder. ..................... 25

Figure 2.19 | Laser writing setup to study AOS...................................................... 26 
Figure 2.20 The beam diameter was measured by cutting the beam with a blade as the light intensity was recorded. FWHM of the derivative provides the beam diameter.

Figure 2.21 | "Salt-and-pepper" structure due to thermal demagnetization (arrow), associated with random up and down magnetization direction.

Figure 2.22 | AOS stripe shown by arrows. See figure 3.2 (b) for uniform switched stripes

Figure 2.23 | Different shapes of magnetic domains. Orange arrows show where the stripes are written. Red arrows show the magnetic domains............................... 28

Figure 2.24 | Magneto-optical pump-probe experiment. ............................................ 30

Figure 2.25 | An example to describe a pump-probe experiment. Assume a ferromagnetic sample with $\mathrm{M}$ along Heff (a). A laser pulse (pump) is incident on the sample at $\tau=0$ leading to a new effective field Heff', which causes $M$ to start precessing. If the probe beam arrives at the sample before the pump it would detect $\mathrm{M}$ as it is in (a). A probe with delays 50 ps or 120 ps would result in cases (b) or (c). 50 and 120 ps delay times correspond to 14 and $36 \mathrm{~mm}$ respectively of extra distance that the probe pulse has to travel compared to the pump pulse.

Figure 2.26 | Time-resolved MOKE measurement on Ni/Si sample. ............................. 32

Figure 2.27 | Comparing pump-probe measurements in reflection and transmission geometries for a $\mathrm{Co} / \mathrm{Pd}$ sample on glass substrate. 33

Figure 2.28 | While thermal contributions were large, the magnetic signal was much smaller and harder to measure. To address this issue we kept the polarizer and analyzer almost at crossed angles (the middle curve), which minimizes the thermal contribution (symmetric peak or dip) to the signal.

Figure 2.29 | Measurements at the correct analyzer angle results in distinct symmetric and anti-symmetric components, representing the thermal and magnetic parts of the

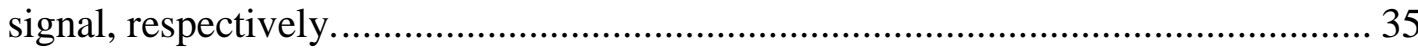

Figure $3.1 \mid$ (a) L- and P-MOKE hysteresis loops at room temperature for samples A and B. (b) Sketch of the writing setup. 39

Figure 3.2 | (a) Background domains of sample A. (b) AOS in sample A at $150 \mathrm{~mW}$ power. (c) Nucleation of secondary domains at stripe center in sample A at $125 \mathrm{~mW}$ power and smaller speed.

Figure 3.3 | Polarizing microscopy images of sample B. (a) AOS domains extend away from under the laser beam profile (top) and show complementary contrast at small stripe spacing (bottom). All stripes were written with $600 \mathrm{~mW}$ power, LP light, and at $10 \mathrm{~mm} \mathrm{~s}^{-1}$ speed. (b) Opposite reversal across a stripe. Writing parameters are (380 $\mathrm{mW}, \mathrm{LCP}, 1 \mathrm{~mm} \mathrm{~s}^{-1}$ ) for 1, (220 mW, LCP, $\left.1 \mathrm{~mm} \mathrm{~s}^{-1}\right)$ for 2, (600 mW, LP, $10 \mathrm{~mm} \mathrm{~s}^{-1}$ ) for 3 and 4 . 
Figure 3.4 | MFM (a) and polarizing microscopy (b) images of a stripe in sample B (100 $\mathrm{mW}, \mathrm{LP}, 10 \mathrm{~mm} \mathrm{~s}^{-1}$ ). The arrows show the domain walls. Inset: sketch of a side view of the magnetic structure.

Figure 3.5 | Domain walls are oriented differently, depending on the scan direction. The arrows show the direction of laser beam motion. Writing parameters for stripe 1, 2, and 3 were (sample B, $600 \mathrm{~mW}$, LCP, $10 \mathrm{~mm} \mathrm{~s}^{-1}$ ), (sample A, $125 \mathrm{~mW}$, RCP, $10 \mathrm{~mm}$ $\mathrm{s}^{-1}$ ), and (sample A, $190 \mathrm{~mW}, \mathrm{RCP}, 10 \mathrm{~mm} \mathrm{~s}^{-1}$ ), respectively.

Figure 3.6 | Magnetic dots made with chopped beams show a delay in the onset of reversal. These stripes were written in sample A. Writing parameters for (a)-(c) were $\left(\mathrm{f}=20 \mathrm{~Hz}, 230 \mathrm{~mW}, \mathrm{LCP}, 5 \mathrm{~mm} \mathrm{~s}^{-1}\right),\left(\mathrm{f}=10 \mathrm{~Hz}, 230 \mathrm{~mW}, \mathrm{LCP}, 2.5 \mathrm{~mm} \mathrm{~s}^{-1}\right)$ and $\left(\mathrm{f}=10 \mathrm{~Hz}, 230 \mathrm{~mW}, \mathrm{RCP}, 10 \mathrm{~mm} \mathrm{~s}^{-1}\right)$, respectively, where $\mathrm{f}$ is the chopping frequency. The arrows show the direction of laser beam motion. The top panel shows the heat accumulation at the center of a Gaussian beam.

Figure $3.7 \mid$ Frame at $\mathrm{t}=37 \mathrm{~ms}$ from results of numerical calculations of the temperature variations of the glass substrate with Dglass $=0.005 \mathrm{~cm} 2 \mathrm{~s}$, for a $\mathrm{F}=5 \times$ $107 \mathrm{Wm} 2$ fluence focused in an area $\mathrm{A}=30 \mu \mathrm{m} \times 30 \mu \mathrm{m}$. The colour scale is in degrees $\mathrm{C}$. The panels show the time-dependence of the temperature and in-plane heat flux at point $\mathrm{A}$ on the surface during the beam scan.

Figure 4.1 | (a) Left panel: $\mathrm{T}_{\mathrm{e}}, \mathrm{T}_{\text {latt }}$ and $\mathrm{M}$ of an ultrafast model for $\mathrm{P}_{\mathrm{abs}}=40 \mathrm{~mW}$ and $\mathrm{h}=4.1 \mathrm{~nm}$ with bulk cobalt parameters $[63,65,76]$, neglecting heat accumulation and electron-induced UDM. Right panel: sketch of the magnetization timedependence in GdFeCo with AOS domains starting after $\sim 10$ ps at the center [91]. This takes a ms-range time interval in cumulative AOS of $\mathrm{Co} / \mathrm{Pd}[88]$ and $\mathrm{Co} / \mathrm{Pt}$ [86]. (b) Sketch of the experimental setup. Inset: autocorrelation of $190 \mathrm{fs}$ pump pulses at the sample location

Figure $4.2 \mid \mathrm{R}(\tau)$ for different magnetic fields and incident pump beam power. ............. 63

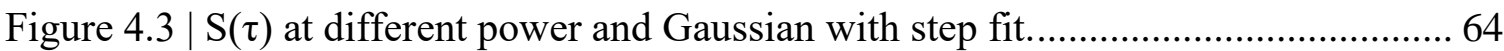

Figure $4.4 \mid \mathrm{A}(\tau)$ at different power and two-exponential fit.

Figure 4.5 | (a) Initial random spin distribution in a $50 \mathrm{~nm}$ radius cylinder. Spins pointing up or down out of plane are labelled blue and red. (b) Examples of a MD final state, $\mathrm{S}$ state as domains walls expand outward beyond the radius of the initial cylinder, IP state with spins in-plane, and NC state with all spins pointing up. (c) Simulation results for $M_{s}=1500 \times 10^{3} \mathrm{~A} / \mathrm{m}, \mathrm{h}=10 \mathrm{~nm}$, and $\mathrm{R}=50 \mathrm{~nm}$. (d) For $M_{\mathrm{s}}=500 \times 10^{3} \mathrm{~A} / \mathrm{m}$, $\mathrm{h}=10 \mathrm{~nm}$ and $\mathrm{R}=50 \mathrm{~nm}$.

Figure 4.6 ( a) Sketch of a demagnetized cylinder of radius $\mathrm{R}$ and height $\mathrm{h}$ made by the laser beam. (b) Side view of a plane with an uniform PMA magnetic structure with $\mathrm{HD}=4 \pi M_{s}$ inside and no field lines outside. (c) Field lines for a reversed cylinder, where additional (dotted) fields decrease $\mathrm{H}_{\mathrm{D}}$ compared to the next case. (d) Field lines for a DM cylinder. (e) Dependence on R/h of DM fields at the center and edge of a demagnetized or reversed cylinder. The field at other points is in-between these 
two limits. Fields of a partially demagnetized cylinder can be obtained by combining these cases. 72

Figure 4.7 Image sequence of the magnetization time-evolution for one $\mathrm{S}$ point in figure 5 (b) at $\mathrm{K}=1800 \times 10^{3} \mathrm{~J} / \mathrm{m}^{3} ; \mathrm{A} / \mathrm{a}^{2}=2400 \times 10^{3} \mathrm{~J} / \mathrm{m}^{3} ; \mathrm{M}_{\mathrm{s}}=1500 \times 10^{3} \mathrm{~A} / \mathrm{m}$. Field of view zooms out at $\mathrm{t}=21$ to a larger area.

Figure 5.1 | (a) Sketch of the pump-probe setup. Inset: the response of the sample symmetric in applied field $\mathrm{S} \tau=12(\mathrm{~S}+\mathrm{B}+\mathrm{S}-\mathrm{B})$ measured at $\mathrm{fch}=2.069 \mathrm{KHz}$. (b) The scanning setup with the $\mathrm{Co} / \mathrm{Ag}$ sample thickness profile in the inset. 79

Figure 5.2 (a) Temperature modulation amplitude $\mathrm{T} \omega$ dependence on $\mathrm{fch}=\omega \mathrm{ch} 2 \pi$, normalized to the value at $30 \mathrm{~Hz}$, for $\mathrm{Co} / \mathrm{Pd}(40 \mathrm{~mW}$ incident power), $\mathrm{Co} / \mathrm{Ag}(46$ $\mathrm{mW})$, and $\mathrm{Co} / \mathrm{Au}(40 \mathrm{~mW})$. Similar results were obtained for other powers between $20 \mathrm{~mW}$ and $60 \mathrm{~mW}$. (b)Phase dependence on chopper frequency fch. (c)Light transmission is higher at stripe locations and correlates with variations in $\mathrm{T} \omega$ across $\mathrm{Co} / \mathrm{Ag}$ stripes.

Figure 5.3 | (a) Vertical stripe made in $\mathrm{Co} / \mathrm{Ag}$ across a stack of horizontal stripes, with distances shown in $\mu \mathrm{m}$. Integrated intensity profiles show the stripe almost completely disappears when inside the stack, while the edges are relatively unaffected. (b) Structural birefringence at stripe edges. (c)End of stripe for $\mathrm{Co} / \mathrm{Au}$ at different incident power shows the steady-state condition is not obtained during scanning.

Figure 5.4 | (a) Conducting interface (case A) with cooling rates limited by the substrate. (b)A resistive interface (case B) limits the cooling rate. (c) Two layer model, with a metallic and a glass film. 88

Figure 5.5 | Amplitude (a) and phase (b) of the temperature modulation for different G. As expected, the amplitude $\mathrm{T}$ increases from $\mathrm{A}$ to $\mathrm{B}$. The parameters are Pabs $=$ $30 \mathrm{~mW}, \mathrm{~d}=30 \mathrm{~nm}$, weff $=200 \mu \mathrm{m}$, Ds $=5 \times 10-7 \mathrm{~m} 2 \mathrm{~s}, \Lambda \mathrm{s}=1 \mathrm{WmK}, \mathrm{Df}=0.1 \times$ $10-4 \mathrm{~m} 2 \mathrm{~s}, \Lambda \mathrm{f}=100 \mathrm{WmK}$ (c) Results with a glass layer dl with $\mathrm{Dl}=0.05 \mathrm{Ds}, \Lambda \mathrm{l}=$ $0.05 \Lambda \mathrm{s}$, weff $=200 \mu \mathrm{m}$. Similar results are obtained for layers with the same Dldl2 ratio. The case of $\mathrm{dl}=6 \mu \mathrm{m}$ is shown for $\mathrm{G}=105 \mathrm{Wm} 2 \mathrm{~K}$ (dashed) and $\mathrm{G} \rightarrow \infty$ (solid red line). 92

Figure 5.6 (a) Film $\mathrm{T}_{\max }$ during a scan with power modulated across the horizontal stripes (dashed features). (b) The steady-state solution at vs $=0$ compared to a profile induced by a beam moving with vs $=1.10 \mathrm{~mm} / \mathrm{s}$ shows the larger radius at the end of the scan. (c) Steady-state temperature radial temperature profiles for interface conductances G shown and Pabs $=30 \mathrm{~mW}, \mathrm{~d}=30 \mathrm{~nm}, \mathrm{w} 0=200 \mu \mathrm{m}$, Ds $=5 \times 10-7 \mathrm{~m} 2 \mathrm{~s}, \Lambda \mathrm{s}=1 \mathrm{WmK}, \mathrm{Df}=0.1 \times 10-4 \mathrm{~m} 2 \mathrm{~s}, \Lambda \mathrm{f}=100 \mathrm{WmK}$ without the glass layer, compared to the 3D case (thick metal film), and with results including the same glass layer as in figure 5(c). The difference between results for $\mathrm{G}=106 \mathrm{Wm} 2 \mathrm{~K}$ and $\mathrm{G} \rightarrow \infty$ is negligible. The right axis shows the Gaussian beam fluence profile. 
Figure 6.1 | Variation of $\mathrm{M}_{\mathrm{RE}}, \mathrm{M}_{\mathrm{TM}}, \mathrm{M}_{\text {total }}$ and coercive field $\mathrm{H}_{\mathrm{c}}$ with temperature...... 102 Figure 6.2 | Decrease of the coercive field Hc with increasing the temperature from $130 \mathrm{Oe}$ at $100 \mathrm{~K}$ to $40 \mathrm{Oe}$ at $225 \mathrm{~K}$

Figure 6.3 | L-MOKE measurements performed on the GFC sample with variable thickness showed two typical hysteresis loops. A normal (a) and an inverted (b) hysteresis loop. The inset in (a) shows the P-MOKE result. 105

Figure 6.4 | MOKE measurements performed on the sputter deposited $[\mathrm{Co} / \mathrm{Pd}]_{4}$ sample with variable thickness in (a) Longitudinal and (b) \& (c) Polar modes. The red wedge represents the thickness variation in the sample. The loop in (a) was obtained at the thickest end of the sample, (b) and (c) were obtained at two different spots on the thinner parts of the sample with out of plane magnetization. 106

Figure 6.5 | Hysteresis loops measured on CFA/Si(111) samples of 18nm (left) and 36nm (right) thicknesses in longitudinal and polar geometries. The result illustrates an inplane easy axis in the thicker sample. The hysteresis loops of the thinner film do not match an in-plane or out-of-plane easy axis and the magnetization may be at an angle. Since CFA is biaxial with three different magnetic axes, the analysis of the hysteresis behavior is not as straightforward as in a uniaxial material. 107

Figure 6.6 | Magnetization acquired at constant applied magnetic field. The sample was rotated 360 degree during this measurement. Different colors represent different field magnitudes. The $50 \mathrm{~nm}$ CFA sample was sputter deposited on a $\mathrm{Si}(111)$ substrate. 108

Figure 6.7 | Illustration of a modified chopper wheel to create sensitivity to signal derivatives, with glass pieces attached to introduce an additional delay in the probe beam path. Then, measuring the signal at the glass frequency shows variations as the glass pieces modulate the delay, analogous to measuring the signal derivative $\Delta \mathrm{I} / \Delta \tau$.

Figure 6.8 | Using the delay chopper is equivalent to taking derivative of the standard signal. This measurement was performed on a $[\mathrm{Co} / \mathrm{Pd}]_{4}$ sample with a $350 \mathrm{~mW}$ pump beam.....

Figure 6.9 | Structures created during writing with high power laser on $6 \mathrm{~nm}$ thick $\mathrm{CoPd} / \mathrm{glass}$. The orientation of ripples/rims depends on writing direction (arrows). Features are visible in both AFM (color) and polarizing microscopy (gray scale) images (of different stripes) (a) The laser power employed for writing the $1^{\text {st }}$ stripe was $700 \mathrm{~mW}$ at $\mathrm{v}=2.5 \mathrm{~mm} / \mathrm{s}$. $2^{\text {nd }}$ stripe is written with $\mathrm{P}=800 \mathrm{~mW}$ at $\mathrm{v}=$ $1 \mathrm{~mm} / \mathrm{s}$. For the vertical stripe (a) $\mathrm{P}=600 \mathrm{~mW}$ and $\mathrm{v}=2.8 \mathrm{~mm} / \mathrm{s}$ and (b) $\mathrm{P}=700$ $\mathrm{mW}$ at $\mathrm{v}=2.8 \mathrm{~mm} / \mathrm{s}$.

Figure 6.10 | Stripes written at different laser powers on 4nm-thick CoPd/glass. Arrows show the writing direction. (a) The writing speed increases slightly along the stripe and less heat is deposited. First stripe shows no ripples at the right side. Third stripe is written at low power and did not create ripples. (b) More pulses and larger 
absorbed heat created larger ripples at the beginning of the stripes (top): $\mathrm{P}=620 \mathrm{~mW}$ at $\mathrm{v}=10 \mathrm{~mm} / \mathrm{s}$.

Figure 6.11 | Periodic features created during writing at high power on CoPd/glass of variable thickness. (a) High power features are more pronounced on the left side of image (a) as the film was thicker on this side leading to higher heat absorption and more damage. (b) The stripe is written at $1000 \mathrm{~mW}$ and is present along most of the sample. Image (c) shows the same stripe at higher resolution. The periodicity of the features depends on laser power and writing speed.............................................. 113

Figure 6.12 | Writing at different laser power on CoPd/glass of variable thickness....... 113 Figure 6.13 Polarizing microscope image in reflection mode of a stripe and dot in $\mathrm{CoPd} / \mathrm{glass}$ sample of variable thickness, showing details of the pattern created in the glass. 


\section{CHAPTER 1: INTRODUCTION}

\subsection{Ultrafast demagnetization (UDM)}

It was not until mid-nineties that ultrafast demagnetization (UDM) was demonstrated using femtosecond laser pulses [1]. This came as a surprise, as it was previously accepted that magnetization could not be modified faster than the precession period (Figure 1.1) [2].

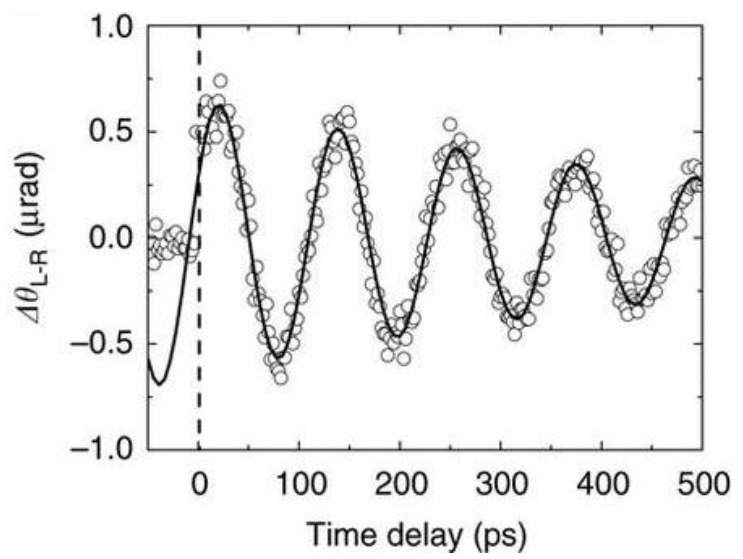

Figure 1.1 | Timescale of magnetization precession [3]. The plot shows polar MOKE results of a $\mathrm{Co} / \mathrm{Au}$ sample. The $\mathrm{y}$-axis denotes polarization rotation and is proportional to the component of magnetization perpendicular to the sample surface.

Measurements showed that UDM takes less than a picosecond, approximately two orders of magnitude faster than precession periods (Figure 1.2). Laser induced UDM was measured using pump-probe techniques, where a femtosecond laser beam is split into 
pump and probe beams, each made of a sequence of pulses. The role of the probe pulse is to quantify changes in the sample properties triggered by the pump pulse. To do this, a probe pulse is made to arrive at the sample at a certain delayed time after the pump pulse, with the timing between the two pulses adjusted using a translation stage on the probe beam path.

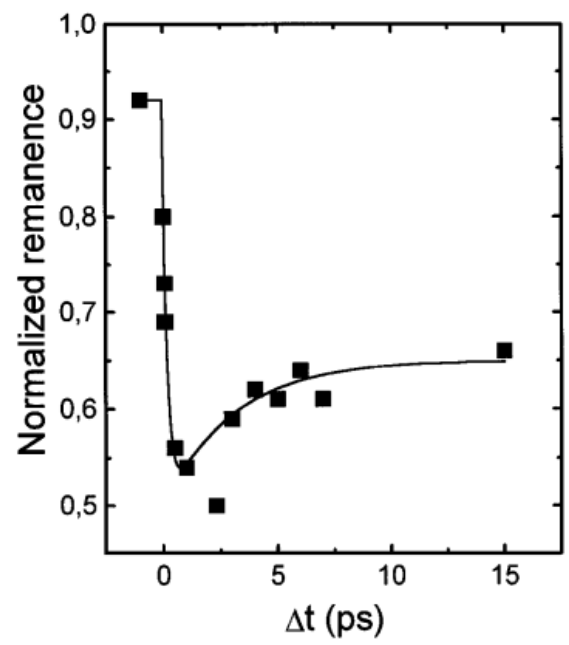

Figure 1.2 | First demonstration of ultrafast demagnetization in a Ni sample [1]. The timedependence of remanent magnetization (the magnetization at zero applied field), measured with a pump-probe experiment, shows a sudden drop in magnetization within 260 fs of laser excitation. The result was normalized to the signal obtained without the pump beam.

The signal obtained with this technique can be related to the sample magnetization because of the magneto-optical effect, which involves a rotation of the polarization angle of an incident beam upon its reflection or transmission through a material. Since even nonmagnetic birefringent materials can change the polarization angle, it is convenient to define a total rotation $\theta_{T}$ as the sum of a magnetization-induced rotation $\theta_{A}$ and a nonmagnetic contribution $\theta_{S}$. The magnetic part $\left(\theta_{A}\right)$ of the signal is 
what we are looking for, as it is proportional to the sample magnetization. An accurate account of the magnetic ordering in a sample requires careful separation of the magnetic and nonmagnetic components of the signal (section 2.6).

Observing UDM triggered a debate on the interpretation of the results [4]. Considering the contribution of optical transients to the signal, it is a legitimate question to ask whether the drop in Figure 1.2 can be interpreted as demagnetization. The UDM timescale was later confirmed with a time-resolved experiment by detecting the $\mathrm{THz}$ electromagnetic wave emitted upon UDM [5].

It is worthwhile to note that lasers with longer pulses did not show similar demagnetizing effects. For example, lasers with 5-20 ps pulses did not induce UDM in a Ni sample, even though the power was high enough to increase the film temperature to twice the Curie temperature [6]. On the other hand, using 40 ns laser pulses resulted in irreversible demagnetization of the sample [6]. A recent study explored the influence of pulse length on the demagnetization and demonstrated that UDM is obtained only with pulses shorter than 1 ps [7].

Understanding the UDM process requires examining the non-equilibrium magnetic state triggered by femtosecond laser pulses. Different models were developed to explain UDM, including a microscopic three-temperature model (M3TM) [8]. This model indicates that UDM is due to flipping of electron's spin upon a collision with phonons (spin-flip scattering). This occurs during heat transfer between three reservoirs: electrons, spins and phonons (lattice vibrations). 


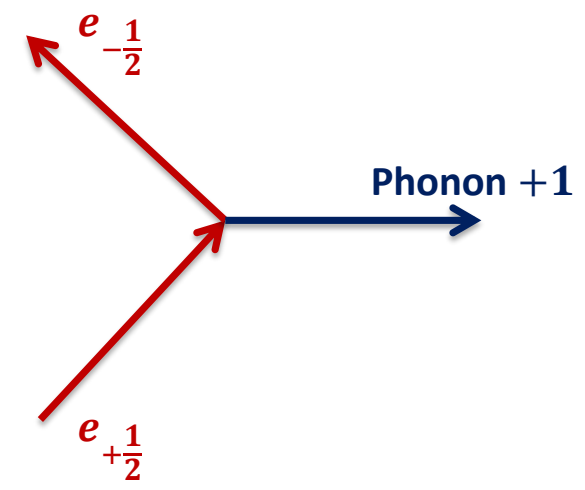

Figure 1.3 | The M3TM model describes UDM as the result of multiple spin-flip scatterings that occur during the temperature equilibration of electrons with the lattice [7]. Initial models suggested that UDM is due to the increase of temperature (since the magnetization reduces as the temperature increases toward Curie temperature). However, magnetization dynamics associated with transfer of angular momentum should be taken into account and the microscopic $3 \mathrm{~T}$ model indicated that UDM results from dissipation of electron's angular momentum into the lattice.

\subsection{All-optical switching (AOS)}

The surprising observation of ultrafast demagnetization stimulated a growing field of research in laser-induced magnetization dynamics. An appealing aspect of UDM was its reversibility, in contrast to the long-pulsed lasers that could demagnetize only at the cost of permanently removing the ferromagnetic properties. Could the non-damaging laser pulses reverse the magnetization as well?

The first observation of laser-induced magnetization reversal without an assisting magnetic field, was reported in 2007 [9] on a GdFeCo (GFC) sample (Figure 1.4) and was called all-optical switching (AOS). It was also achieved later in ferromagnetic materials. Switching the magnetization with ultrafast lasers was an important achievement because it meant overcoming the time limit previously observed in 
switching with ultra-short high intensity fields [2]. The timescale of AOS was recently determined in a GFC sample to be within 100 ps from pulse excitation (Figure 1.5). Thus, AOS appeared as a promising technique for data writing and storage applications.

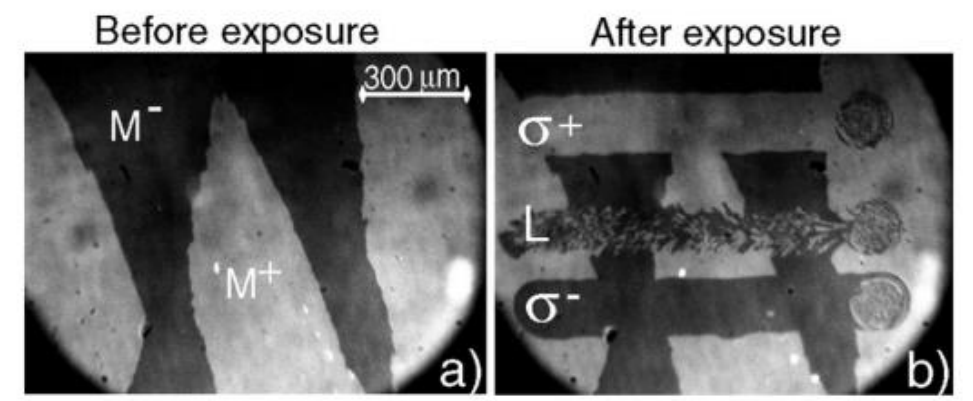

Figure 1.4 | Magneto-optical images of GFC (a) before and (b) after scanning under a pulsed laser beam [9]. L, $\sigma^{+}$and $\sigma^{-}$denote the linear polarization, right-handed and lefthanded circularly polarization, respectively. Dark (bright) areas correspond to domains with down (up) magnetization.

The initial result on GFC showed helicity-dependent reversal, suggesting a link between reversal and the magnetic field induced in the material by the circular polarization of the beam. However, later studies showed that the reversal in GFC could take place even with linearly polarized light [10], pointing to the role of heat in magnetization switching. The preferred approach to address AOS in GFC and other ferrimagnets (atoms with unequal and opposing magnetic moments) is with an atomistic model that attributes the reversal to exchange interactions and to the difference in magnetic behavior of the $\mathrm{Gd}$ and $\mathrm{Fe} / \mathrm{Co}$ (the rare earth and transition metal components) sub-lattices with temperature. This model was further supported by measurements of magnetization time evolution of Gd and Fe sub-lattices separately as the reversal emerges (Figure 1.6). 

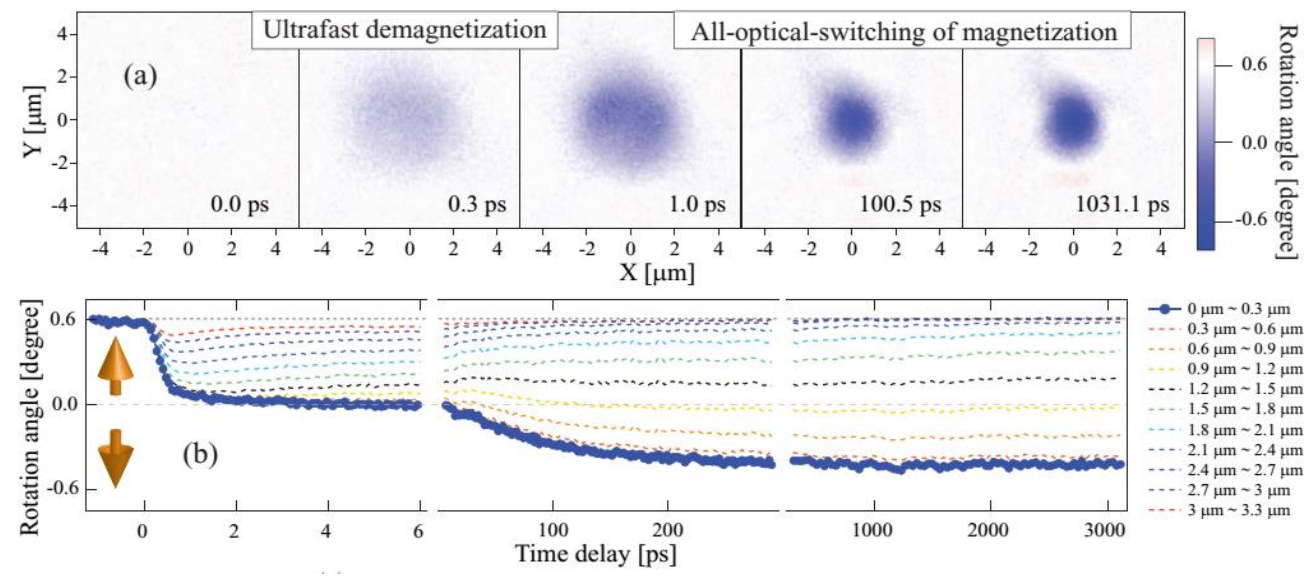

Figure 1.5 | Time-dependence of magnetization during AOS in a GFC sample, measured with pump-probe microscopy [11]. This result revealed that AOS in GFC develops on a 100 ps timescale after pump pulse excitation. The y-axis in the lower plot shows the polarization rotation, which is proportional to magnetization. The angle change from positive angle to negative confirms magnetization reversal.

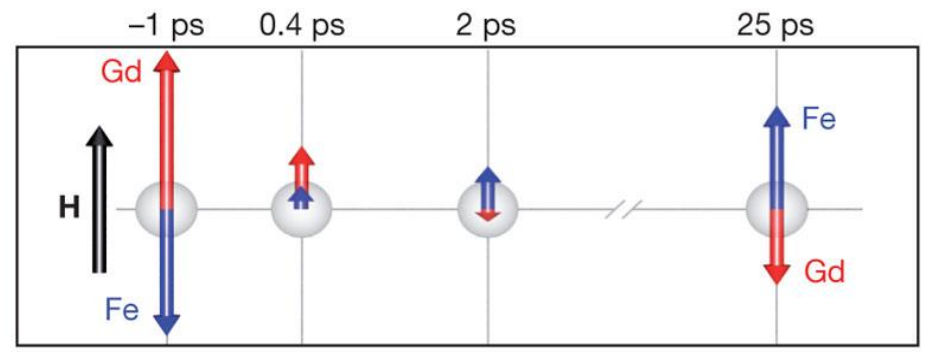

Figure 1.6 | Time evolution of magnetization of individual Gd and Fe sub-lattices [12]. In the proposed AOS mechanism in ferrimagnet GFC, reversal emerges through a transient ferromagnetic-like state, in which the magnetic moments of rare earth (RE) and transition-metal (TM) elements are parallel.

Interestingly, while a single pulse in GFC can lead to AOS (figure 1.7), multiple pulses must be applied in other samples (for instance, $\mathrm{Co} / \mathrm{Pt}$ ) to obtain switching. In the experiments presented in this thesis, we examined the influence of the multiple-pulses on switching and magnetization dynamics, as our laser has a high-repetition rate $(80 \mathrm{MHz})$. 


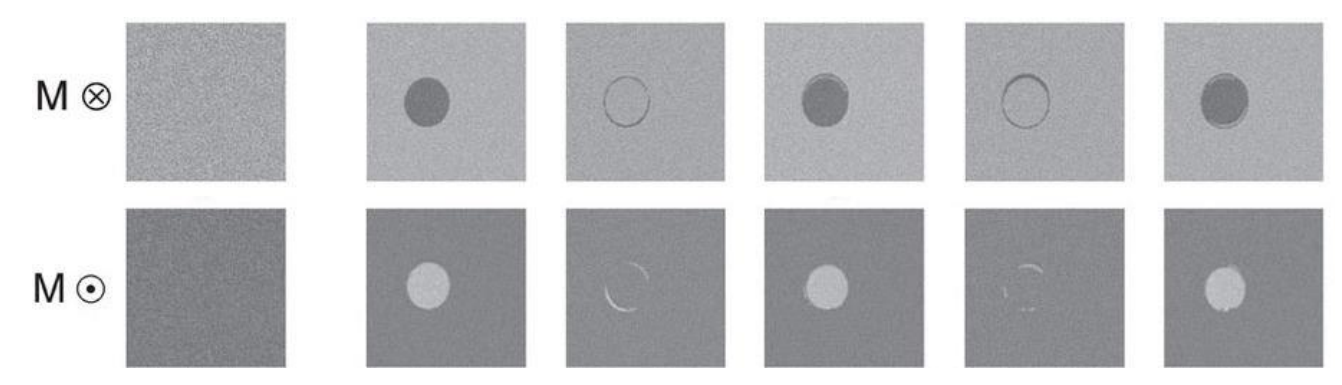

Figure 1.7 | Polarizing microscopy images of AOS with a single pulse in a GFC sample with initial magnetization pointing up (bright background, top row) and down (dark background, lower row) [10].

\subsection{Perpendicular magnetic anisotropy}

In addition to pump-probe experiments that quantify the magnetization dynamics, a static assessment of AOS is possible if changes in magnetization are visible in polarizing microscopy. In the simplest implementation, this requires an out-of-plane magnetization orientation (section 2.2), corresponding to perpendicular magnetic anisotropy (PMA). Magnetic anisotropy refers to preferred magnetization $(\vec{M})$ directions, along one or several easy axes. Alignment of $\vec{M}$ with an easy axis lowers the total magnetic energy of the material.

The easy axis orientation is governed by several magnetic energies of a sample that contribute to the total magnetic energy, including exchange coupling, magnetocrystalline energy, magnetostatic and Zeeman energies. Exchange coupling is responsible for aligning the spins of neighboring atoms in ferromagnets. It is the distinction between ferro- and paramagnetic materials. For instance, exchange energy for two spins is equal to $A(1-\cos \theta)$, where $A$ is the exchange constant and $\theta$ is the angle between spins, and is minimized when the spins are parallel. Magneto-crystalline energy 
refers to the energy required for rotating the magnetization with respect to the lattice axes. For a crystal with uniaxial anisotropy, $E=K_{u} \sin ^{2} \theta$ where $K_{u}$ is the anisotropy constant. Magneto-crystalline anisotropy stems from the coupling of the atom orbitals and crystallographic axes and spin-orbit interaction and is how the lattice influences the magnetization. Shape or magneto-static anisotropy depends on the material shape and domain structure. For instance, the easy axis of a long particle is along its long axis to minimize the demagnetizing fields $H_{d}$ (also called "stray fields") around the material. The demagnetizing field is induced by the magnetic dipole moments in the material. Magnetic domains can also be formed to minimize the demagnetizing field of a material. Finally, the Zeeman energy is related to an external magnetic field $B_{\text {ext }}$ and is proportional to the component of magnetization along $B_{\mathrm{ext}}$.

Even though shape anisotropy favors an in-plane magnetization, observations show that very thin films feature an out-of-plane magnetization or PMA. Moreover, PMA can be induced by heating a sample to slightly below Curie temperature and applying a magnetic field comparable to sample's coercive field in the direction perpendicular to the surface.

PMA materials provide a framework for increasing data storage density by writing perpendicularly. In addition, AOS has the potential to enhance data processing speeds in magnetic recording media. 


\section{CHAPTER 2: EXPERIMENTAL METHODS}

\subsection{Sample preparation and thickness measurement}

Film thickness is a determining factor in obtaining PMA [13]. PMA samples can be made with different methods, including magnetron sputtering and electron-beam evaporation [13]. Both methods function under high vacuum conditions.

In magnetron sputtering, gas ions erode materials from a disk-shape target (Figure 2.1). The detached particles are then sputtered off to the sample substrate, mounted on a rotatable holder. Rotating the substrate during deposition insures uniformity of the film across it. Magnets beneath the target prevent electrons from colliding with the substrate. Depending on the conductivity of the target, different power sources are employed for bombarding the target. A direct current (DC sputtering) of 200-300 volts is applied for conductive target whereas an alternating radio frequency potential ( $R F$ sputtering) is applied in the case of dielectric targets.

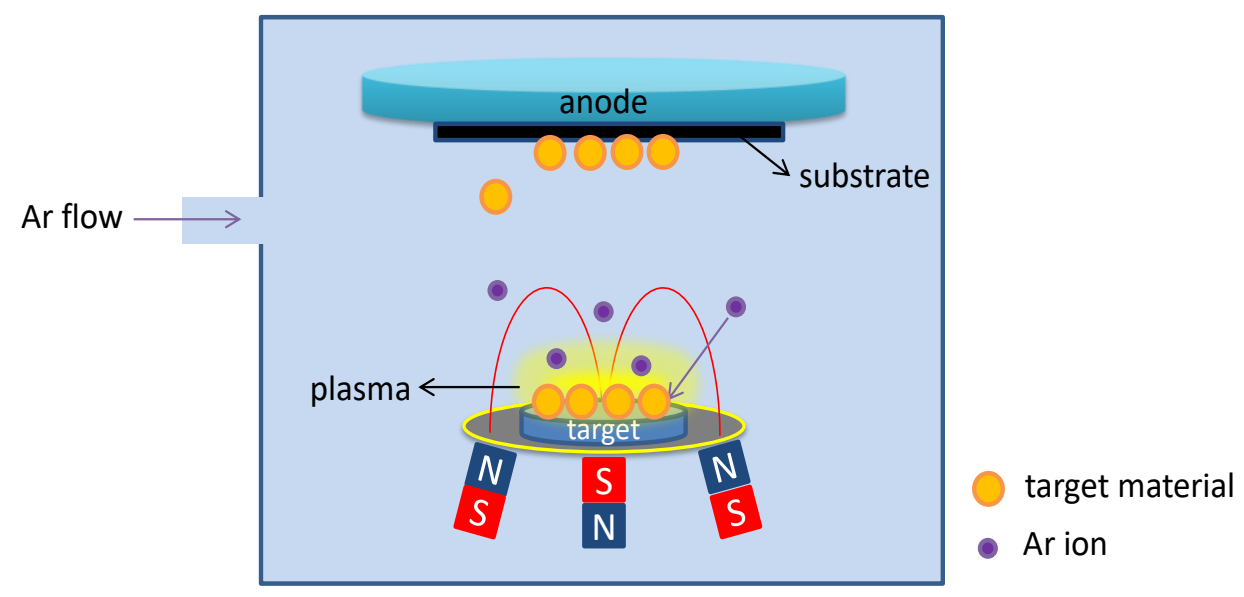

Figure 2.1 | Sketch of a typical magnetron sputtering. 
In E-beam vapor deposition (Figure 2.2), the target pellets are placed in crucibles at the bottom of the vacuum chamber. An electron beam emitted by a filament is guided with electric and magnetic fields to bombard the target materials. Evaporated target material coats the substrate, placed $\sim 50 \mathrm{~cm}$ from the crucibles. To deposit superlattice samples with this method, we had to alternate between crucibles and reheat each before deposition. Choosing the right voltages and currents to steer the electron beam and to expedite the process was crucial.

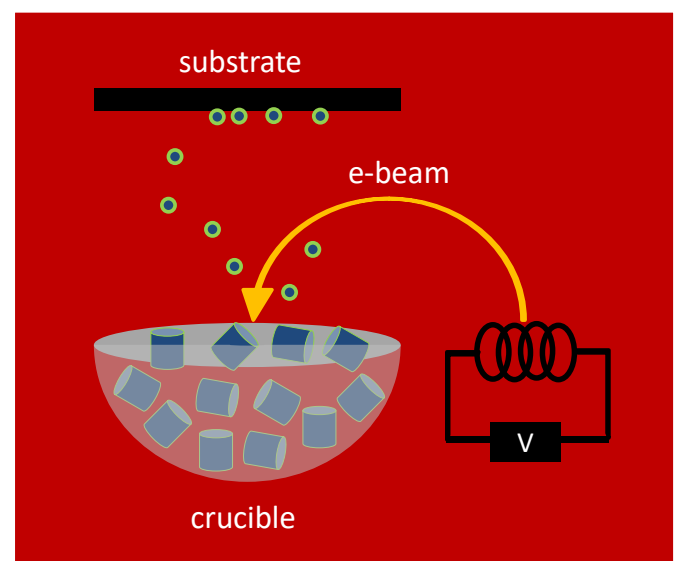

Figure 2.2 | Sketch of electron-beam vapor deposition with pellets of target materials placed in crucibles at the bottom of the vacuum chamber.

We made samples with both variable and uniform thicknesses during each deposition. A holder, consisting of a hood and a wedge, was made for depositing films of variable thickness (Figure 2.3). The hood was placed to partially cover the substrate. Rotating the holder during deposition facilitates thickness variation over a $2 h \tan \theta$ length on the substrate. 


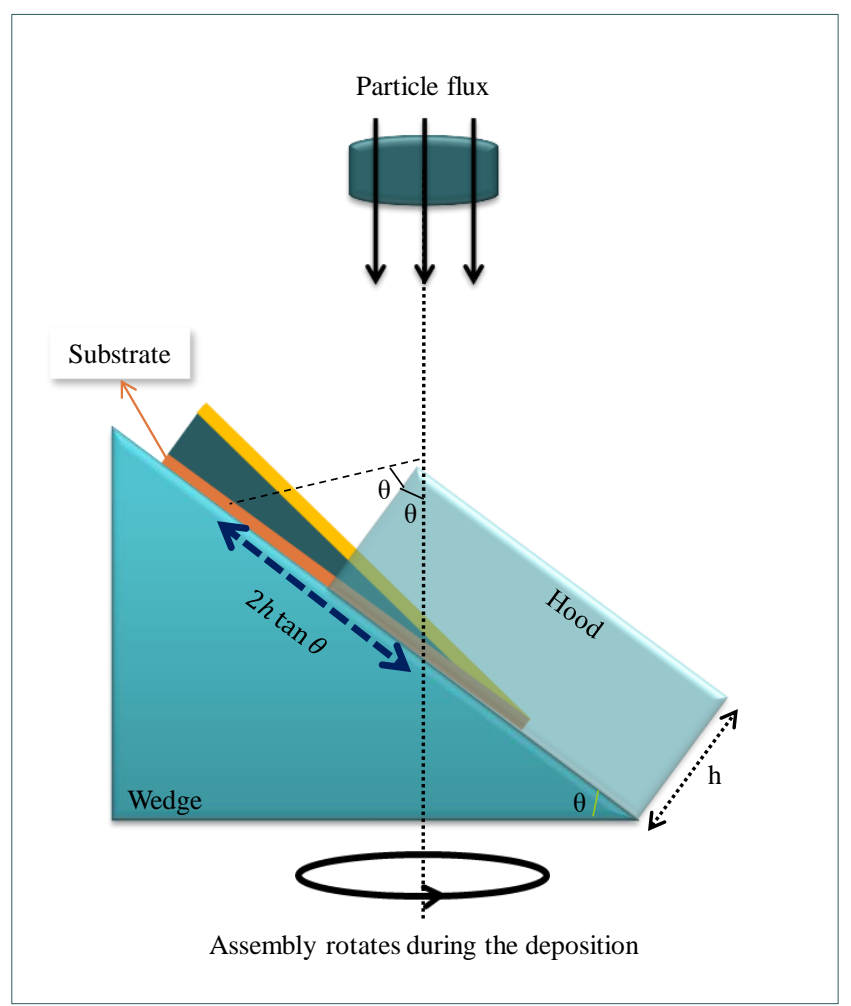

Figure 2.3 | Sample holder used for deposition of films with a variable thickness.

The thickness was measured at the thickest end of the sample with a profilometer, right after the deposition. Absorption measurements were performed using a HeNe laser to confirm the thickness variation. For this purpose, the transmission (T) and reflection (R) off the sample were measured simultaneously as the sample was moved using a motorized stage (Figure. 2.4). Measuring the absorption $\left(A=I_{0}-R-T\right)$ at the same spot, gave the absorption coefficient $\alpha$ calculated using Beer's law $A(t)=I_{0}(1-$ $e^{-\alpha t}$ ), where $I_{0}$ is the laser intensity before reaching the sample and $t$ is the film thickness. Then, the thickness of the thinner parts of the sample was calculated using $\alpha$, $\mathrm{T}$, and R. Figure 2.5 demonstrates the gradual thickness variation in one of the samples deposited using the methods mentioned earlier. The $\mathrm{x}$-axis shows the position on the sample. 


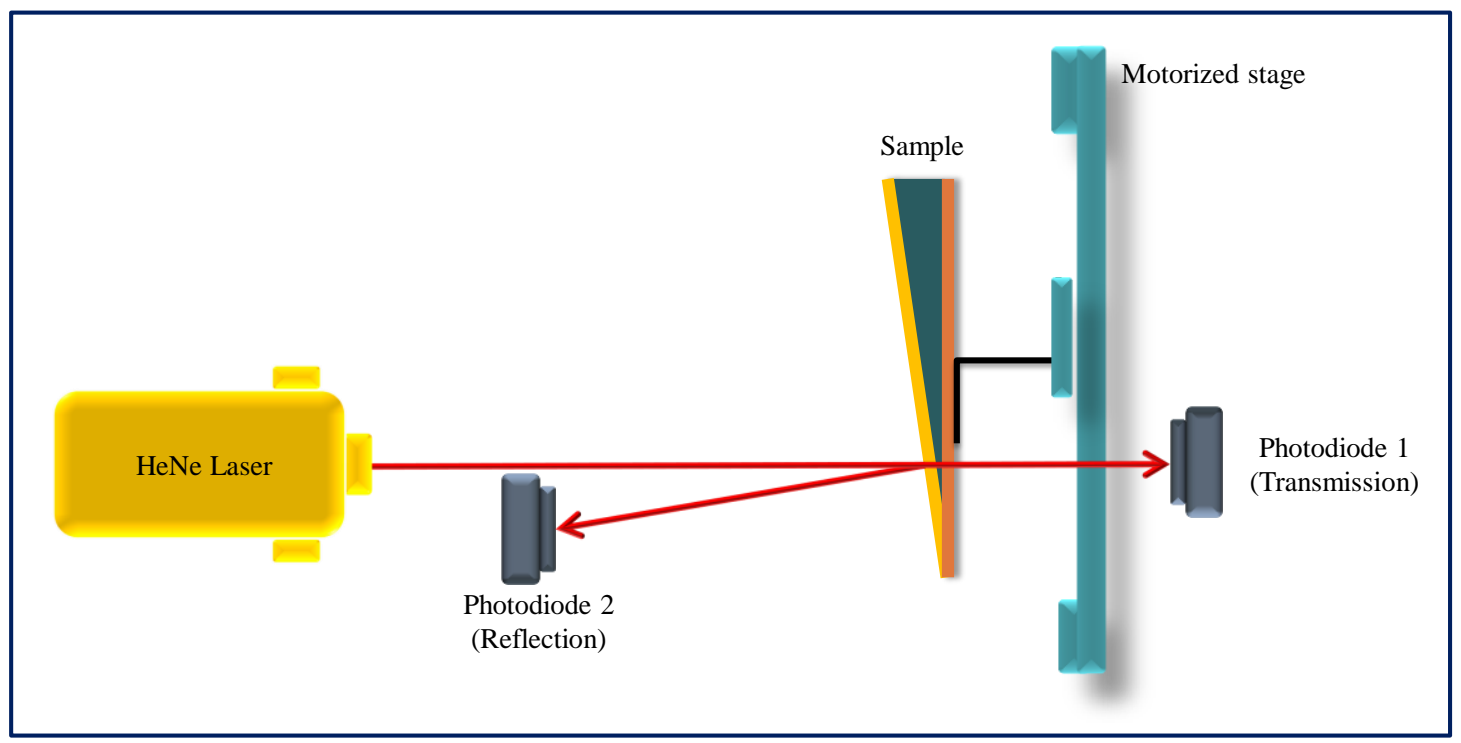

Figure 2.4 | The sample is attached to a motorized stage. A HeNe laser beam is incident on the sample and the intensities of transmission and reflection beams are measured as the stage moves the sample along its length.

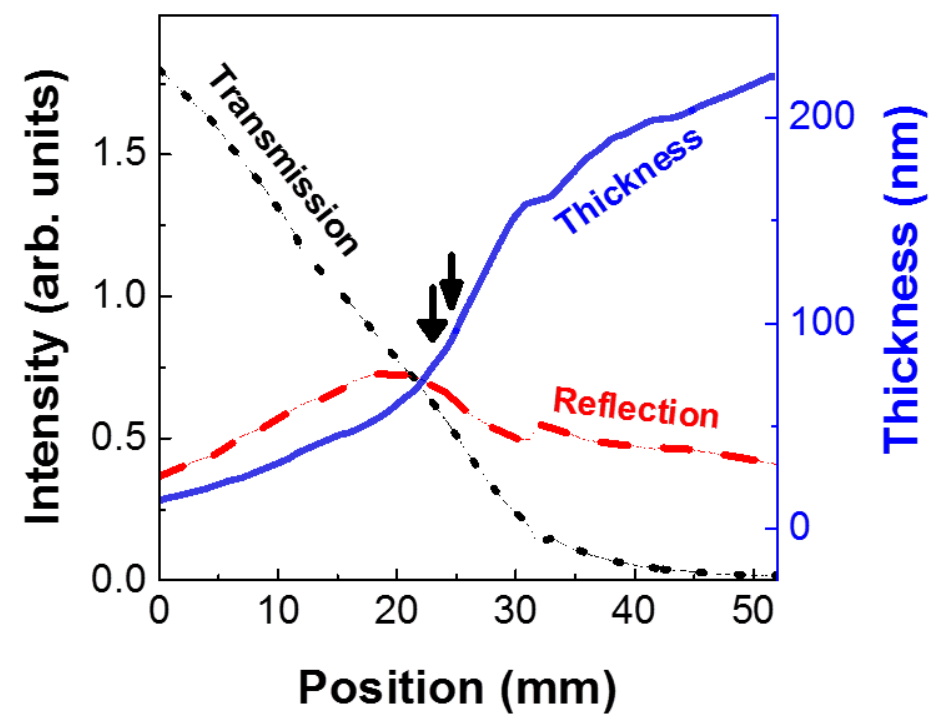

Figure 2.5 | Thickness variation along a GdFeCo (GFC) sample deposited with the method described in figure (2.3). 


\subsection{Polarizing microscopy}

We used polarizing microscopy to image the magnetic structures with out of plane magnetization $\left(\mathrm{M}_{\mathrm{z}}\right)$. It works similar to a regular optical microscope except that it uses a polarizer and analyzer in the light path (Figure 2.6). When they are crossed $(\alpha=90)$ and no sample is in the beam path, light does not reach the detector. Inserting a sample with $\mathrm{M}_{\mathrm{z}}$ between polarizer and analyzer rotates the polarization by a small amount and changes the extinction angle slightly away from 90 degrees.

The polarization rotation is due to the magneto-optical effect that stems from the difference in the speed of left and right circularly polarized (LHC and RHC) light. A linearly polarized light can be considered as the sum of LHC and RHC waves. Their dissimilar speed is associated with differing refractive index for the two, which originates in the dielectric behavior of a material and can be described by a tensor $\epsilon$. For a magnetic sample, the off-diagonal elements of $\epsilon_{i j}$ are proportional to the sample magnetization and their sign changes with magnetization reversal.

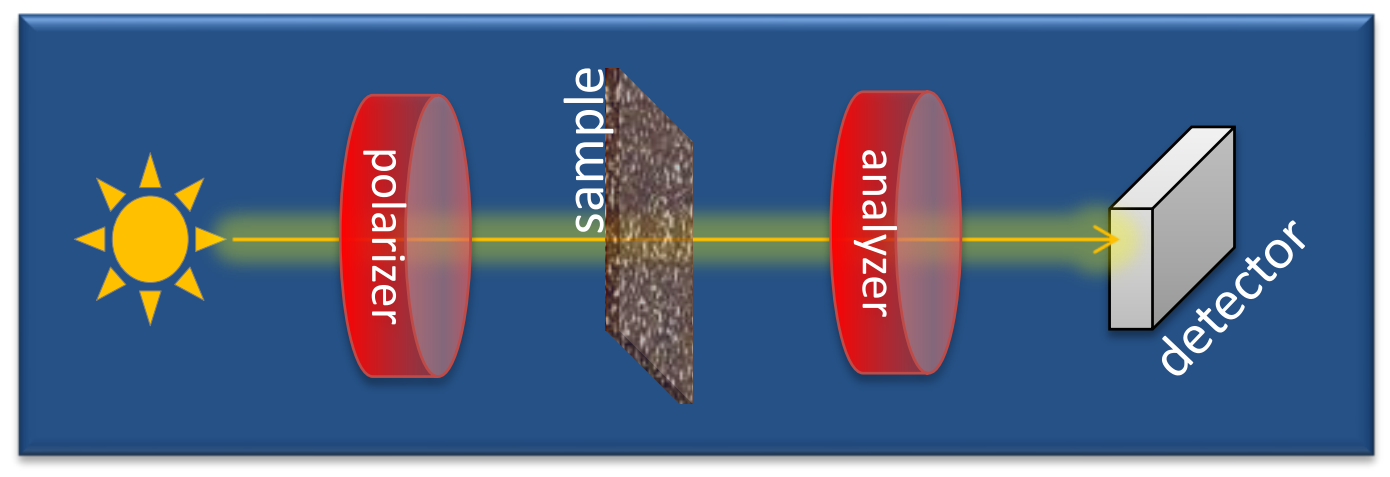

Figure 2.6 | Polarizing microscopy in transmission mode. Depending on sample transparency, it can be used in transmission or reflection mode. 
Demagnetizing the sample with a sinusoidal damped magnetic field (over 20 minutes) led to random up and down magnetic domains observable with a polarizing microscope (Figure 2.7). The domains contrast is reversed when rotating the analyzer angle about extinction angle by \pm 1 degrees. Subtracting the images obtained at analyzer angle -1 and +1 degrees improved the visibility of magnetic domains.

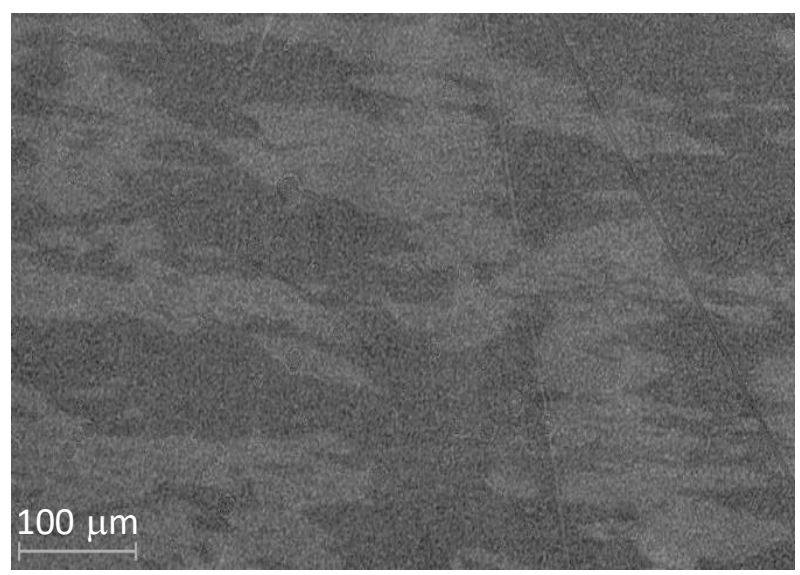

Figure 2.7 | Magnetic domains image produced by subtracting images taken at analyzer angles \pm 1 degrees.

In addition to magnetic domains, we observed a similar contrast change with rotating analyzer in the wings of stripes written on the sample with a Ti:Saphire laser (Figure 2.8). Here, wings refer to the two sides of the stripe where the laser intensity is lower (figure 2.8 and 3.3.a inset). The contrast of both the wings and the laser-induced magnetic domains between the stripes reverses for analyzer angles \pm 1 degree.

The contrast change with analyzer angle can occur due to either optical or magnetic birefringence. One method to distinguish between the two is to rotate the sample under the microscope. The contrast of magnetic features maintains whereas the contrast of areas with optical birefringence varies during the rotation (Figure 2.9). 

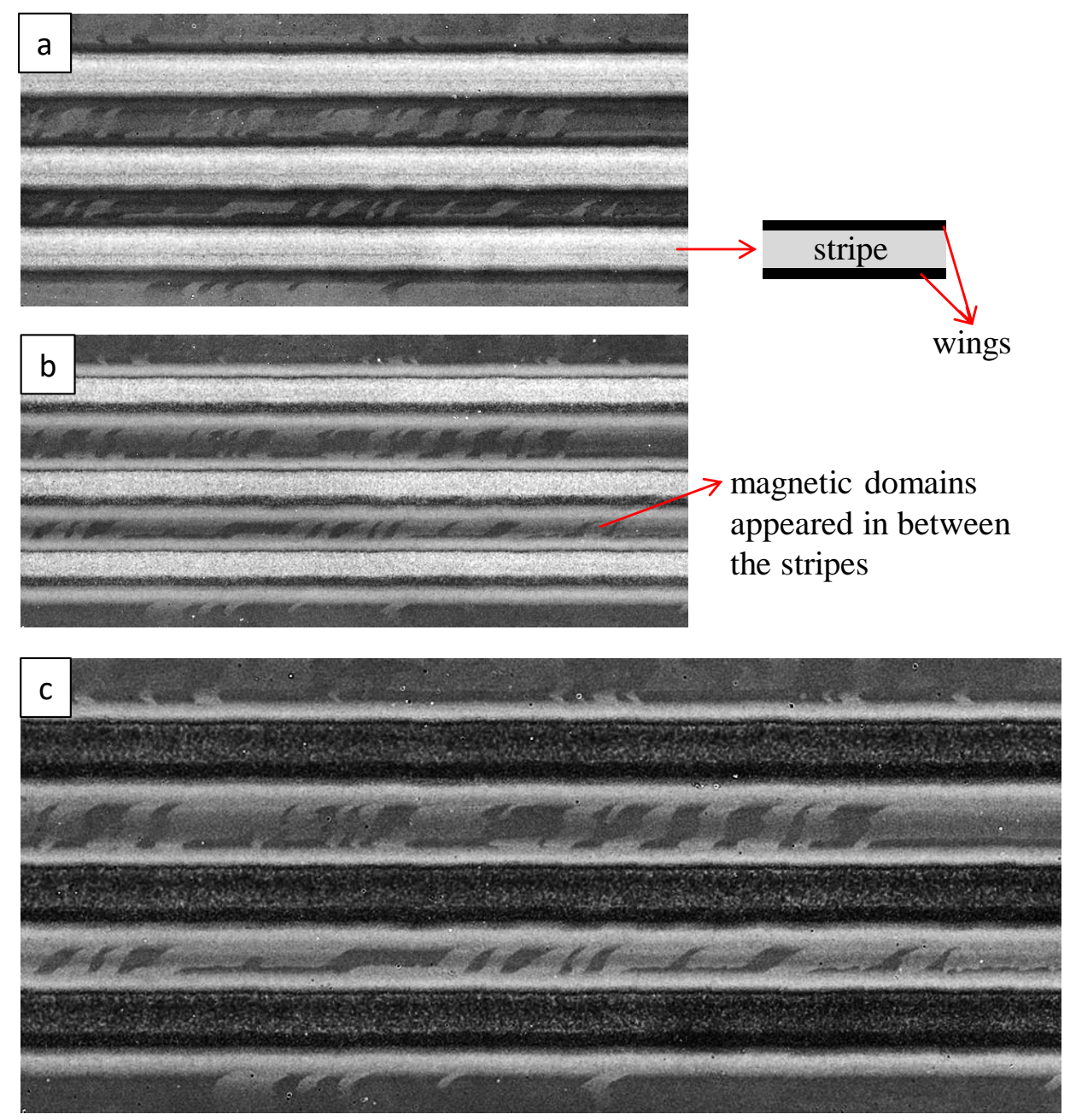

Figure 2.8 | Polarizing microscope images with analyzer angle at -1 (a) and +1 (b) with respect to extinction angle. Image (c) is obtained by subtracting (a) and (b).
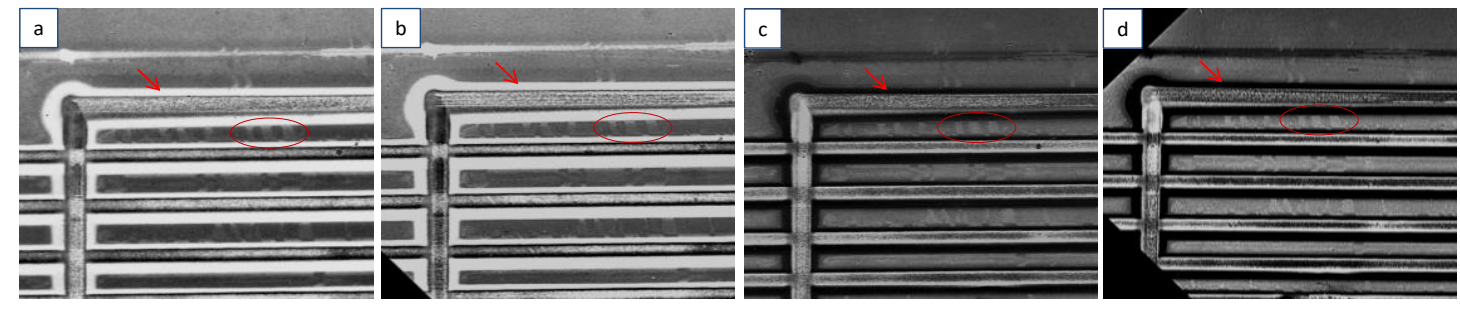

Figure 2.9 | Distinguishing magnetic and optical birefringence. Sample was rotated and imaged at (a) $0^{\circ}$, (b) $45^{\circ}$, (c) $90^{\circ}$, and (d) $135^{\circ}$. The contrast of magnetic domains is fixed, while the wings (arrows) contrast is reversed with angle: bright in (a, b) and dark in (c, d). 


\subsection{Atomic/Magnetic Force Microscopy}

We also applied atomic and magnetic force microscopy (AFM/MFM) for further characterization. AFM is a member of SPM family (scanning probe microscopy). The main advantage of AFM is overcoming the diffraction limit of optical microscopes. It works by detecting the forces between a sharp tip and the surface (Figure 2.10). The tip is typically made of silicon or silicon nitride and is attached to a cantilever. The variations in samples topography lead to changes in the deflection of the cantilever. The final image is formed by sensing the cantilever deflections as the tip is scanned across the surface.

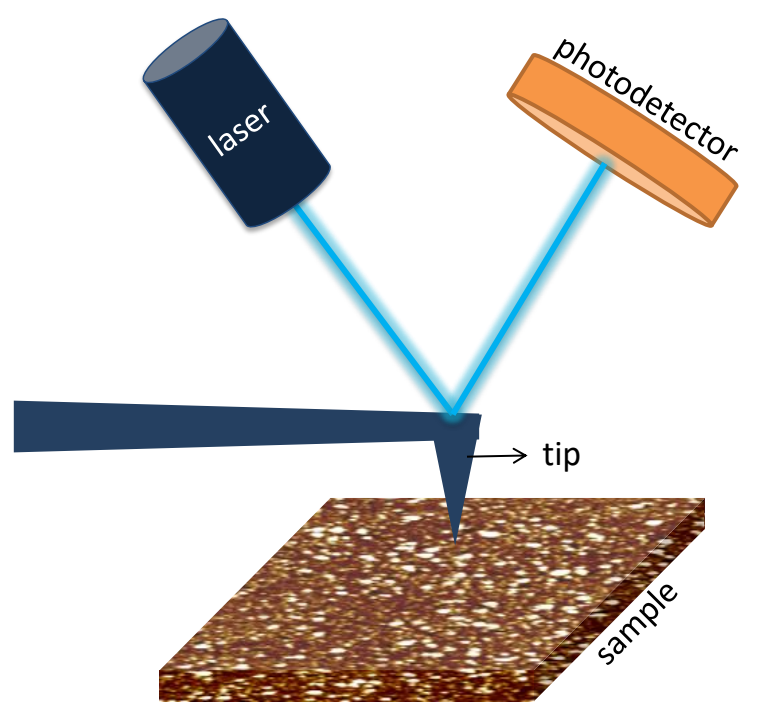

Figure $2.10 \mid$ Atomic force microscope (AFM) works by detecting deflection of a cantilever due to forces between sample and the tip.

For AFM characterization and imaging, we used Asylum MF3D AFM in AC mode. In AC or tapping mode, AFM operates by oscillating the cantilever. Forces from sample influence the cantilever oscillations and the image is formed based on the deviation from driving amplitude, phase or frequency. 
Most of AFMs can be adapted to magnetic force microscopes (MFM) by replacing their tip with a tip covered with magnetic material. This feature enabled us to obtain magnetic images of the samples, complementing the results from polarizing microscope (figure 2.11).

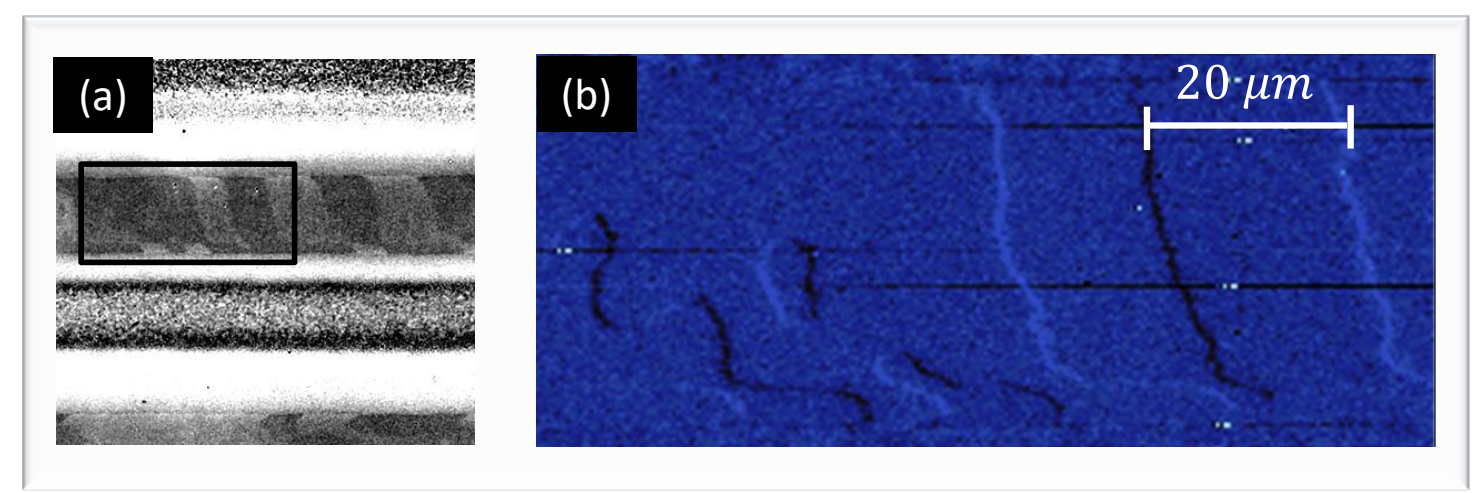

Figure 2.11 | Images of a $\mathrm{Co} / \mathrm{Pd}$ sample acquired by (a) polarizing microscope (b) MFM show the magnetic domains between stripes written with a Ti:S laser.

In magnetic force microscopy, we are interested in magnetic features. To eliminate the topographic structures, the magnetic forces must be distinguished from other forces (for instance, van de Waals forces). A distinctive aspect of magnetic forces is that, even though they are weak, they extend over a relatively long range. Therefore, magnetic imaging should be done at a distance (at least a few nanometers) above the surface, where the magnetic forces are present but the forces responsible for topography are negligible. For this purpose, MFM scans a surface once in the AFM mode to acquire the topography information. Then, taking the topography as the reference, it moves up to a predefined height $\mathrm{h}$ to detect the weak magnetic forces (figure 2.12). 

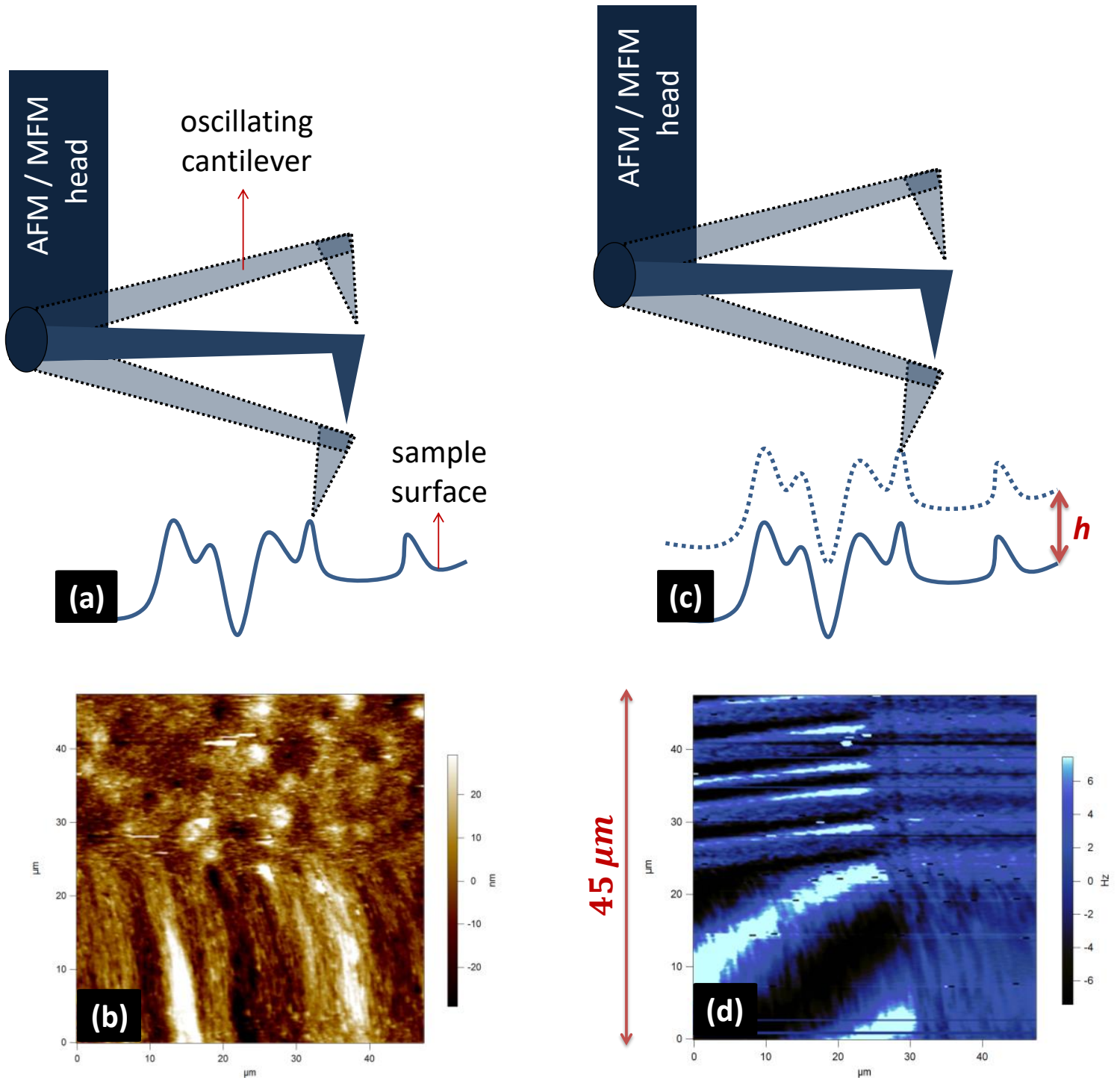

Figure 2.12 | MFM imaging procedure to avoid topography features from contributing to the magnetic image: (a) AFM/MFM head scans the surface to obtain the topography (b) of the surface. (c) The topography data is then used as the reference (the head moves up and down according to topography) for rescanning and detecting the magnetic forces at a predefined height $\mathrm{h}$ where other forces are negligible. (d) Magnetic image of a floppy disc (here $h=50 \mathrm{~nm}, \mathrm{SP}=0.690 \mathrm{~V}, \mathrm{G}=3.5$ ), acquired simultaneously with the topography image (b) of the surface. 
Magnetic force microscopy of a thin film sample with low magnetization can be difficult. With the right parameters, the MFM image would reflect the magnetic structure and the simultaneously acquired AFM would demonstrate the topography of the surface (Figure 2.12). This section reviews the factors that can improve the magnetic imaging with MFM. To find the optimum procedure and parameters for obtaining a good magnetic image, we explored their impact in imaging a floppy disc. We found it crucial to magnetize the tip before imaging. For this purpose, a small magnet attached to a translation stage was kept close to the MFM tip for three minutes. Another important factor for optimizing the magnetic contrast is the distance between the tip and sample $(h \sim 50 \mathrm{~nm})$, which can be set in the software. Two other essential software parameters that affect the results are "set point SP" and "integral gain G". In tapping mode, SP denotes the amplitude of cantilever oscillations and $\mathrm{G}$ reflects the feedback strength.

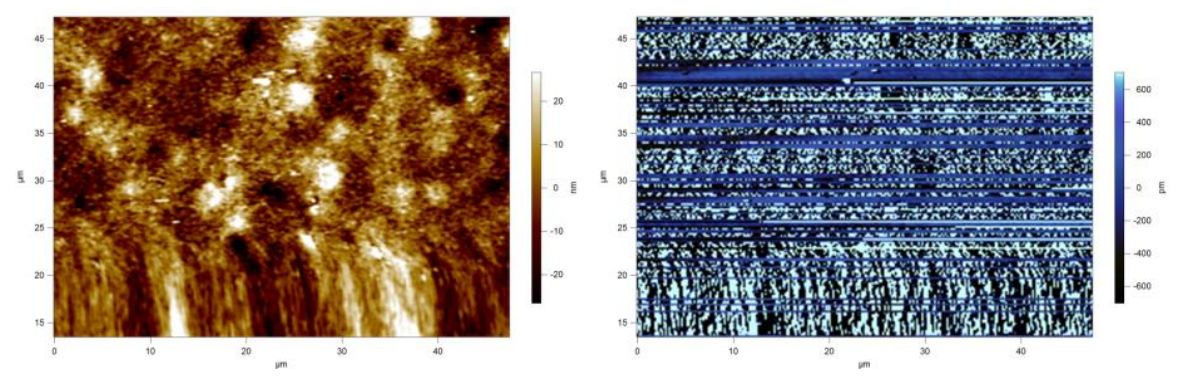

Figure 2.13 | Simultaneously obtained AFM (left) and MFM (right) images of a floppy $\operatorname{disc}(\mathrm{SP}=0.584 \mathrm{~V}, \mathrm{G}=3.5)$.

In general, the integral gain affects the noise level because a high $\mathrm{G}$ increases the oscillations in the image, whereas at a low G, the tip does not sense the surface and the trace and retrace line-profiles do not follow one another. In addition, a high $\mathrm{G}$ leads the magnetic pattern to affect the topography image. 
The set point value should also be optimized. Choosing a low SP causes the topography to influence the magnetic image (Figure 2.13). On the other hand, at high SP values, the magnetic pattern influences the topography image, resulting in a topography image that looks like an alternative of the magnetic image (Figure 2.14). In conclusion, after finding the right $\mathrm{G}$ (when trace and retrace lines overlap), the SP value should be increased if the topography features showed up in the magnetic image and vice versa.

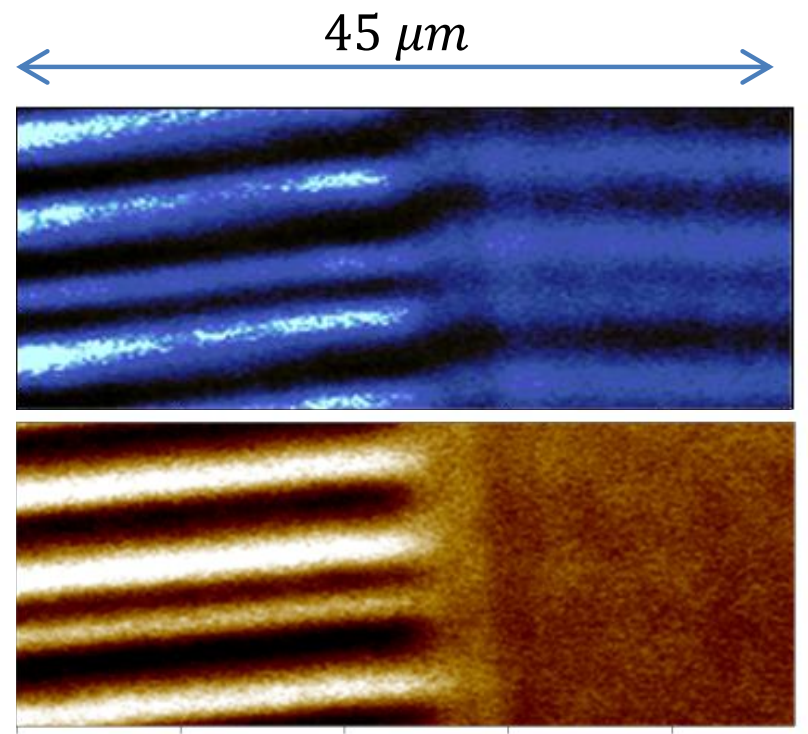

Figure 2.14 | Simultaneously obtained MFM (top) and AFM (bottom) images of a floppy $\operatorname{disc}(\mathrm{SP}=0.776 \mathrm{~V}, \mathrm{G}=3.5)$. 


\subsection{Magneto-optical effect experiments}

To characterize the magnetic properties of the sample, we used magneto-optical Kerr Effect (MOKE) and Faraday Effect experiments. The Magneto-optical Effect refers to the polarization rotation when a polarized beam interacts with a medium. For a magnetic sample, the rotation is proportional to magnetization of the sample. Varying magnetization with an applied magnetic field and measuring the beam polarization after reflecting off or transmitting through a sample provides the magnetization curve (in particular, the hysteresis loop for a ferromagnetic sample) (Figure 2.15).
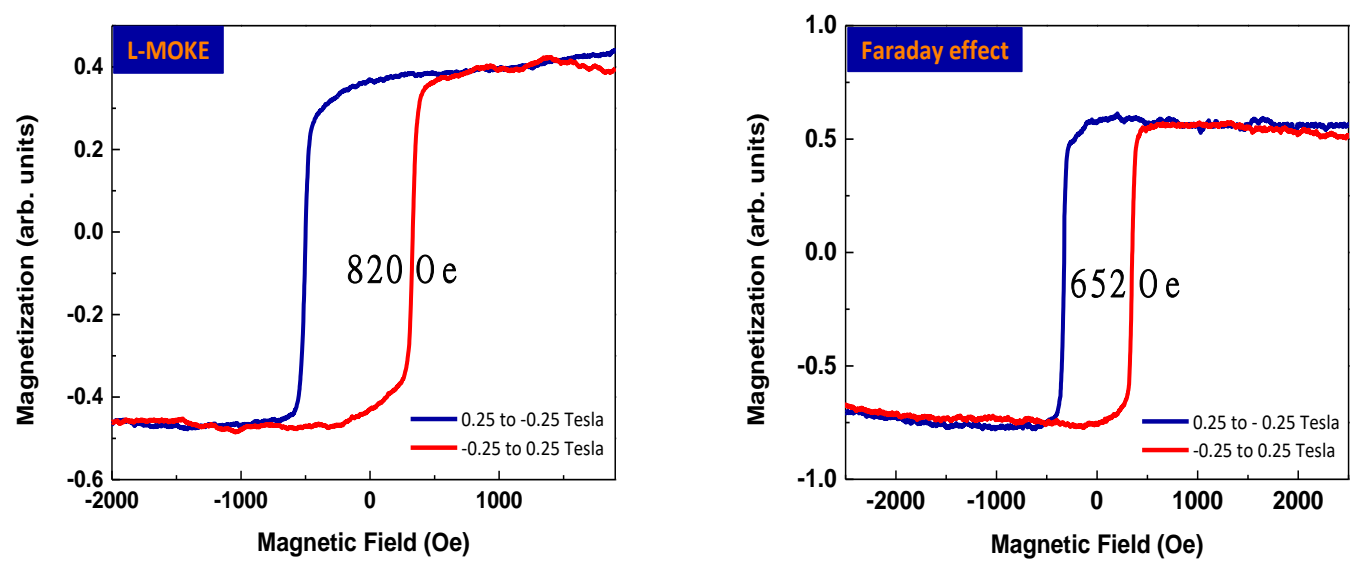

Figure 2.15 Hysteresis loops of a $15 \mathrm{~nm}$ Co film measured using MOKE (left) and Faraday (right) experiments.

The interpretation of MOKE measurements depends on the experimental geometry. Our setup can be interchanged between longitudinal (figure 2.16) and polar modes (figure 2.17). In the longitudinal mode (L-MOKE) the magnetic field is applied along the sample surface. Measurements with L-MOKE setup provide information about in-plane components of the magnetization. In polar mode (P-MOKE) the magnetic field is applied perpendicular to the sample surface. P-MOKE geometry is sensitive to out-ofplane magnetization. 


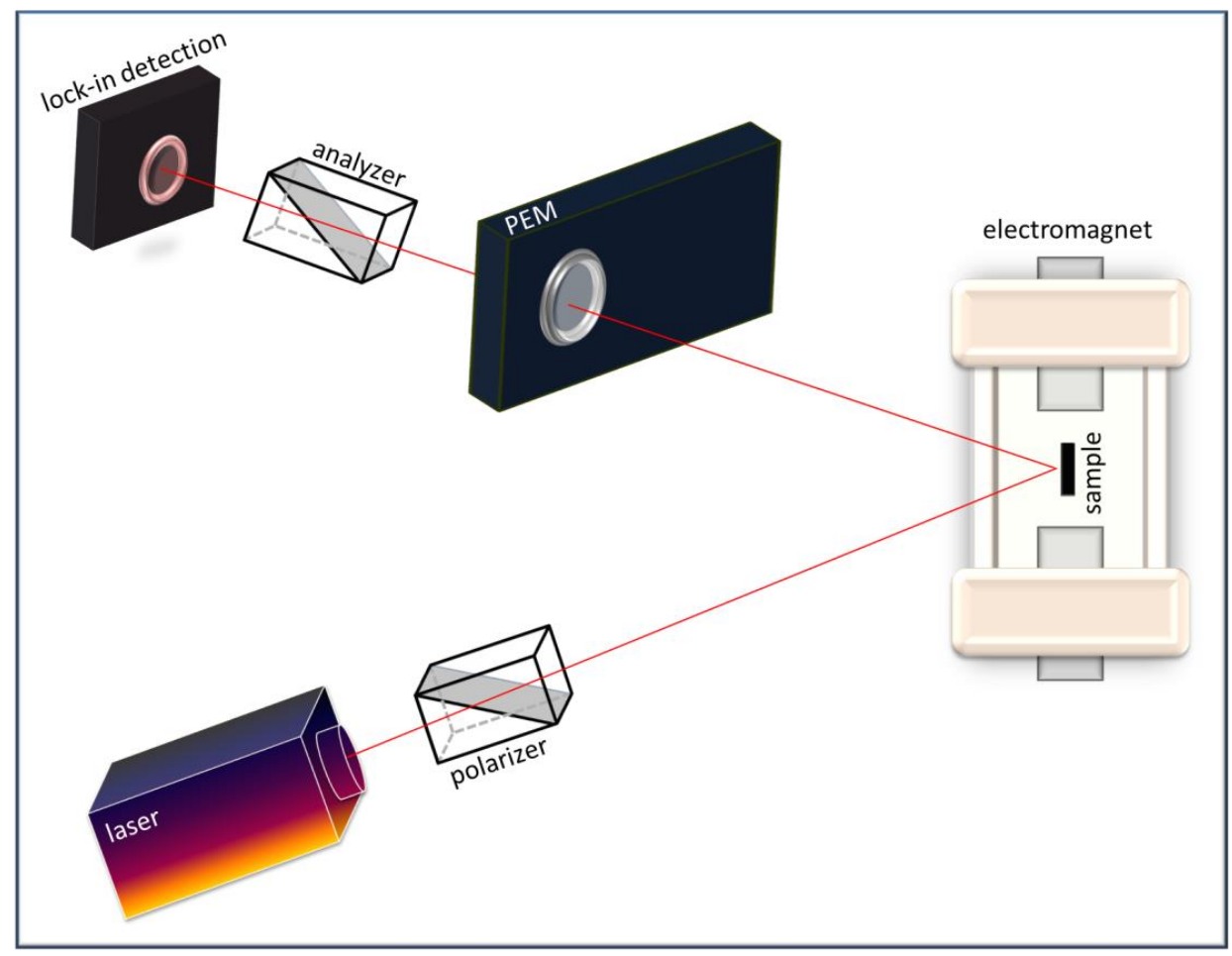

Figure 2.16 MOKE setup in longitudinal geometry. Longitudinal MOKE is used for measuring in-plane components of the magnetization.

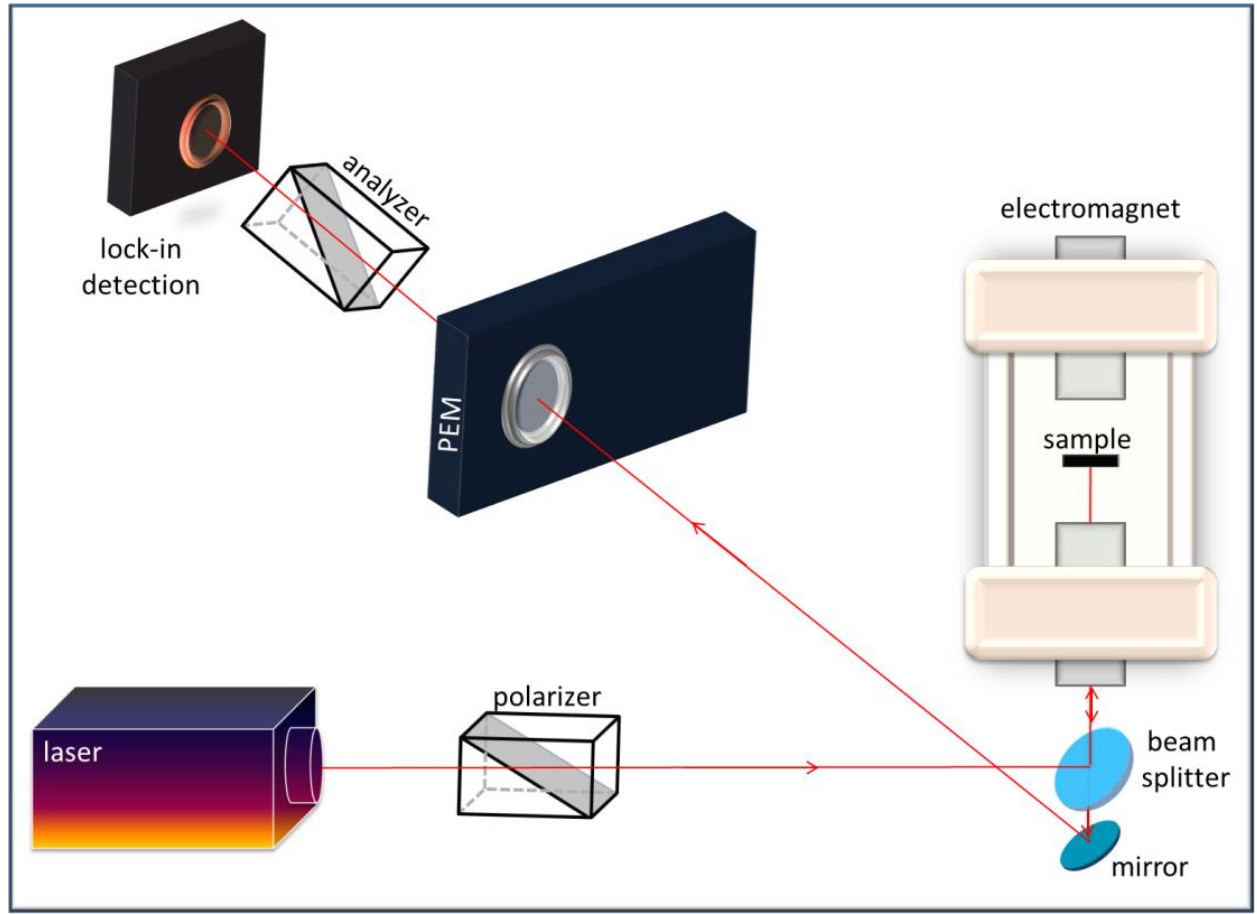

Figure 2.17 | MOKE setup in polar geometry. Polar MOKE is sensitive to out-of-plane components of the magnetization. 
A standard MOKE setup consists of a light source, polarizer, analyzer, magnet and photodiode detection. We use a photo-elastic modulator (PEM) along with lock-in amplifier to overcome the noise. The measured quantity is the light intensity, which is the sum of the square of the electric field components.

Each optical component can be represented by a $2 \times 2$ "Jones" matrix. The final state of the beam that reaches the detector can be represented by a column matrix, whose elements are the electric field components $E_{s}^{\prime}$ and $E_{p}^{\prime}$. It can be obtained by multiplying the matrices of each component the laser beam has passed through as

$$
\left[\begin{array}{c}
E_{p}^{\prime} \\
E_{S}^{\prime}
\end{array}\right]=A \phi S P\left[\begin{array}{l}
E_{p} \\
E_{s}
\end{array}\right],
$$

where $\mathrm{A}, \phi, \mathrm{S}$ and $\mathrm{P}$ are the Jones matrices of the analyzer, PEM, sample and polarizer respectively. Substituting the matrices corresponding to these components we obtain:

$$
\begin{aligned}
{\left[\begin{array}{l}
E_{p}^{\prime} \\
E_{s}^{\prime}
\end{array}\right]=} & {\left[\begin{array}{cc}
(\cos \beta)^{2} & \sin \beta \cos \beta \\
\sin \beta \cos \beta & (\sin \beta)^{2}
\end{array}\right]\left[\begin{array}{cc}
e^{i \varphi(t) / 2} & 0 \\
0 & e^{-i \varphi(t) / 2}
\end{array}\right]\left[\begin{array}{cc}
(\cos \alpha)^{2} & \sin \alpha \cos \alpha \\
\sin \alpha \cos \alpha & (\sin \alpha)^{2}
\end{array}\right] } \\
& {\left[\begin{array}{ll}
r_{s s} e^{i \delta_{s s}} & r_{p s} e^{i \delta_{p s}} \\
r_{s p} e^{i \delta_{s p}} & r_{p p} e^{i \delta_{p p}}
\end{array}\right]\left[\begin{array}{c}
E_{p} \\
E_{s}
\end{array}\right] }
\end{aligned}
$$

where $\alpha$ and $\beta$ are the angles between the incident plane and the major axis of transmission in analyzer and polarizer. $\varphi(t)$ is the phase shift induced by PEM between $\mathrm{E}_{\mathrm{s}}$ and $\mathrm{E}_{\mathrm{p}}$ at a fixed modulation frequency $(50 \mathrm{KHz}$ in our case). The PEM, in conjunction with other elements, allows us to obtain the polarization rotation and ellipticity of the reflected beam. $r_{l m}$ are the Fresnel coefficients of the sample. Multiplying the matrices in equation 1, results in a relation between the detected intensity $I$ and $r_{l m}$. The Fresnel $r_{l m}$ coefficients depend on the dielectric behavior of the material, 
which in case of an anisotropic material, is defined by a tensor:

$$
\epsilon=\left(\begin{array}{ccc}
\epsilon_{x x} & \epsilon_{x y} & \epsilon_{x z} \\
-\epsilon_{x y} & \epsilon_{y y} & \epsilon_{y z} \\
-\epsilon_{x z} & -\epsilon_{z y} & \epsilon_{z z}
\end{array}\right)
$$

As mentioned earlier, the off-diagonal elements $\epsilon_{i j}$ of a dielectric are proportional to magnetization. In fact, $\epsilon_{i j}$ is proportional to the $\mathrm{M}$ component that lies in the direction of $i \times j$, or

$$
\epsilon=\epsilon_{x x}\left(\begin{array}{ccc}
1 & -i Q m_{z} & i Q m_{y} \\
i Q m_{z} & 1 & -i Q m_{x} \\
-i Q m_{y} & i Q m_{x} & 1
\end{array}\right)
$$

where $\mathrm{Q}$ is the magneto-optical constant. This gives a relation between $r_{l m}$ and magnetization components perpendicular $\left(M_{z}\right)$ or along $\left(M_{\|}\right)$the sample [14]:

$$
\begin{gathered}
r_{p s} e^{i \delta_{p s}}=\frac{i n \cos \theta_{i}\left(M_{z} \cos \theta_{r}-M_{\|} \sin \theta_{r}\right) Q}{\left(n \cos \theta+\cos \theta_{r}\right)\left(\cos \theta+n \cos \theta_{r}\right)} \\
r_{p p} e^{i \delta_{p p}}=\frac{n \cos \theta-n \cos \theta_{r}-2 i n \cos \theta_{i} \sin \theta_{r} M_{\perp} Q}{n \cos \theta+n \cos \theta_{r}}
\end{gathered}
$$

We detect different magnetization components depending on the setup geometry. For instance, in L-MOKE with $\alpha=45$ and $\beta=90$, the intensity detected by the photodiode is $I(\omega) \propto\left|r_{p s} r_{s s} \sin \left(\delta_{s s}-\delta_{p s}\right)\right|$. This explains how the in-plane magnetization can be measured with L-MOKE. 


\subsection{Vibrating Sample Magnetometer}

Another method of obtaining the magnetization curves of a sample is vibrating sample magnetometry (VSM). One advantage of using VSM is that it is sensitive to the total magnetic moment of a sample. This is in contrast with MOKE done with visible light $(633 \mathrm{~nm} \mathrm{HeNe}$ laser, in our case), where one can obtain the transition metal magnetization. VSM is in particular useful when the sample surface is not mirror-like, so that the reflection or transmission intensity is too diffuse and weak for Kerr or Faraday measurements.

The sample is placed between the poles of an electromagnet that magnetizes the sample. Vibrating the sample up and down induces changes in the magnetization flux density, leading to induction of an electric field and current in the pickup coils (Faraday law) (Figure 2.18). One can obtain the magnetization curves of the sample by changing the magnetic field and recording the corresponding magnetic moment.

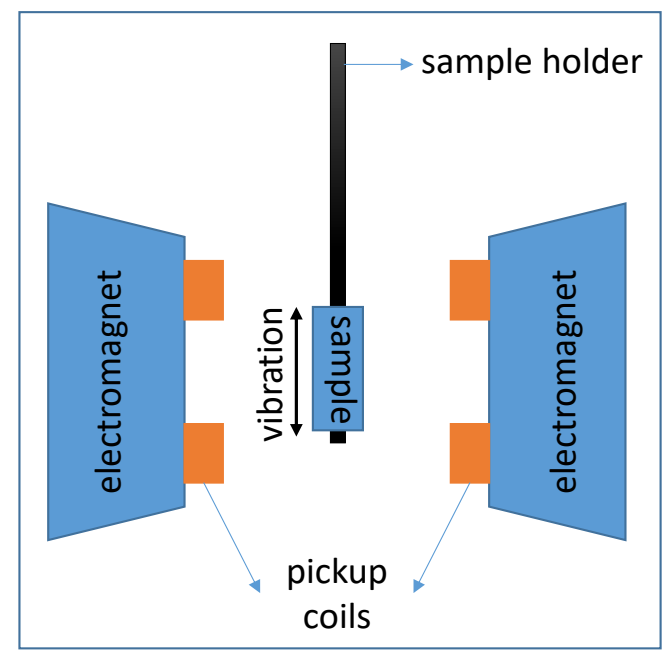

Figure 2.18 | Sketch of vibrating sample magnetometer (VSM). Vibrating a sample in a magnetic field leads to a change in the magnetic flux density. This induces an electric current in four pickup coils surrounding the sample holder. 


\subsection{Laser writing setup}

In AOS experiments, we scanned the samples under an ultrafast TiS laser beam using two motorized stages along two directions orthogonal to the beam (Figure 2.19). The laser fluence was adjusted with a polarizing cube and a half-wave plate (HWP) combination. The beam was focused at the sample with a short focal-distance lens, after passing through a quarter-wave plate (QWP) that sets the beam polarization. The beam diameter was measured by cutting the beam with a blade as the light intensity was recorded. After plotting the intensity versus stage reading, calculating the derivative of the curve resulted in a Gaussian. The beam diameter was obtained from the full width at half maximum (FWHM) of the Gaussian fit (Figure 2.20).

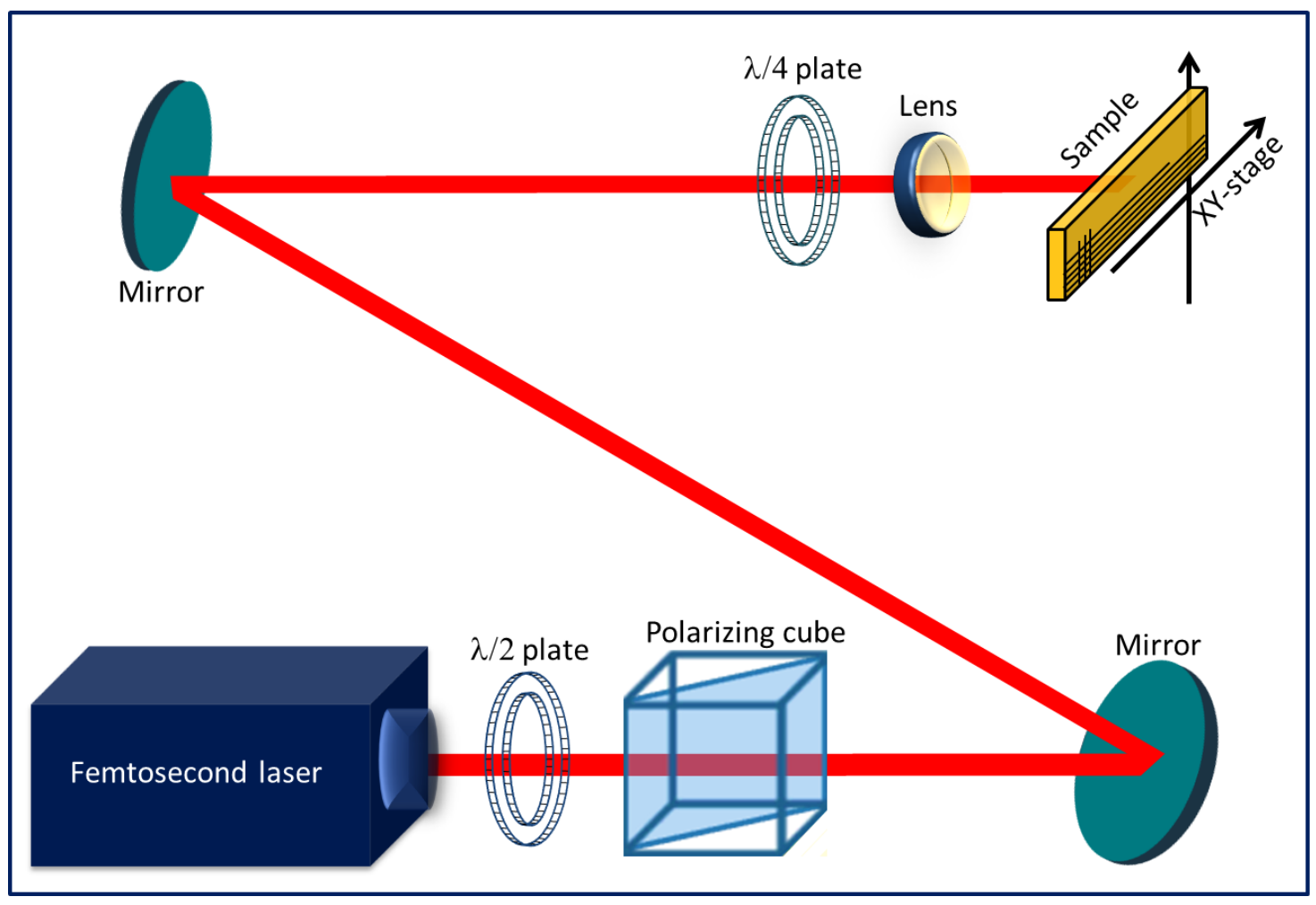

Figure 2.19 | Laser writing setup used to study AOS dependence on fluence and polarization. 

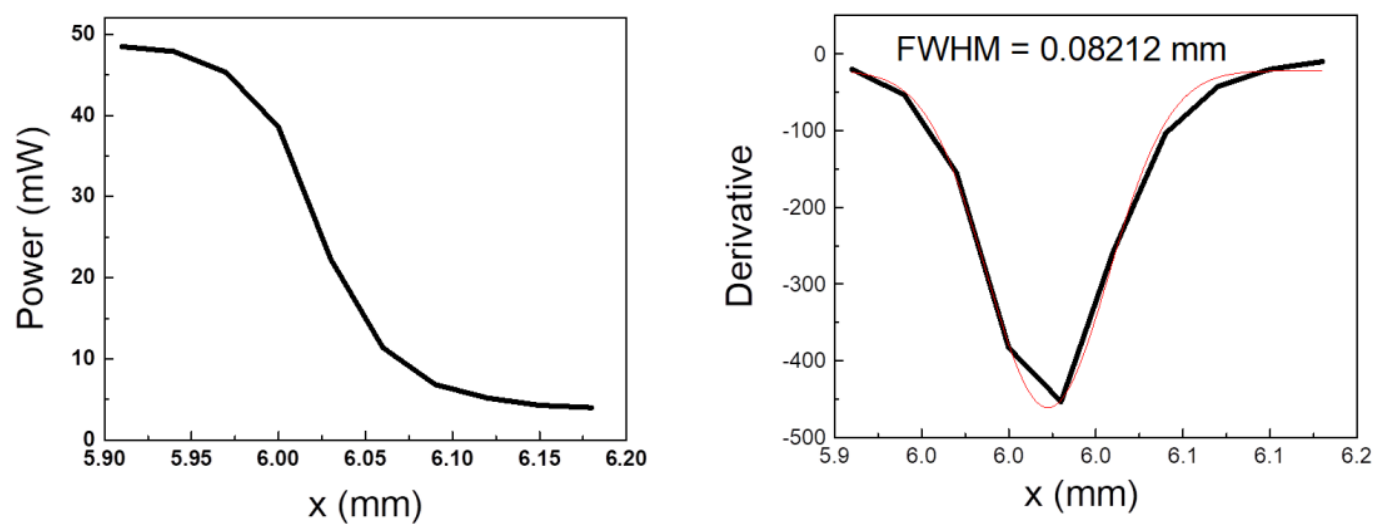

Figure $2.20 \mid$ The beam diameter was measured by cutting the beam with a blade as the light intensity was recorded. FWHM of the derivative provides the beam diameter.

The samples were examined with a polarizing microscope after the writing. Several different final magnetic states were observed, depending on the laser fluence and writing speed $(0.1-10 \mathrm{~mm} / \mathrm{s})$ : high-fluence features (section 6.5), "salt-and-pepper" structure due to thermal demagnetization (Figure 2.21), AOS stripes (Figure 2.22 and 3.2.b) and different magnetic domains (Figure 2.23).

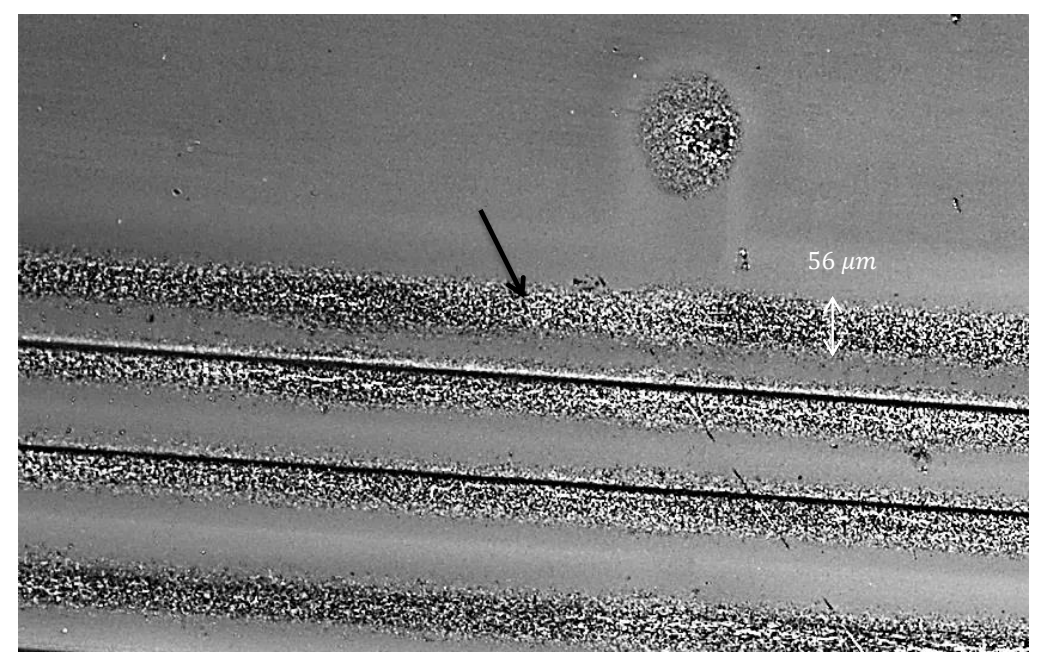

Figure 2.21 | "Salt-and-pepper" structure due to thermal demagnetization (arrow), associated with random up and down magnetization directions. 


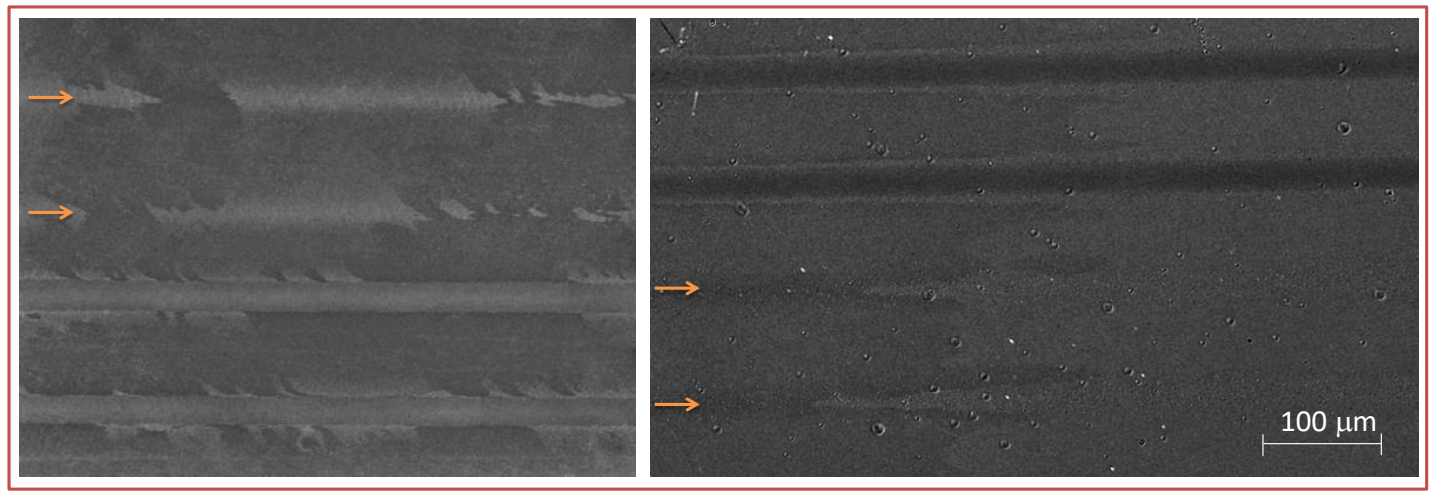

Figure 2.22 AOS stripe shown by arrows. See figure 3.2 (b) for uniform switched stripes.

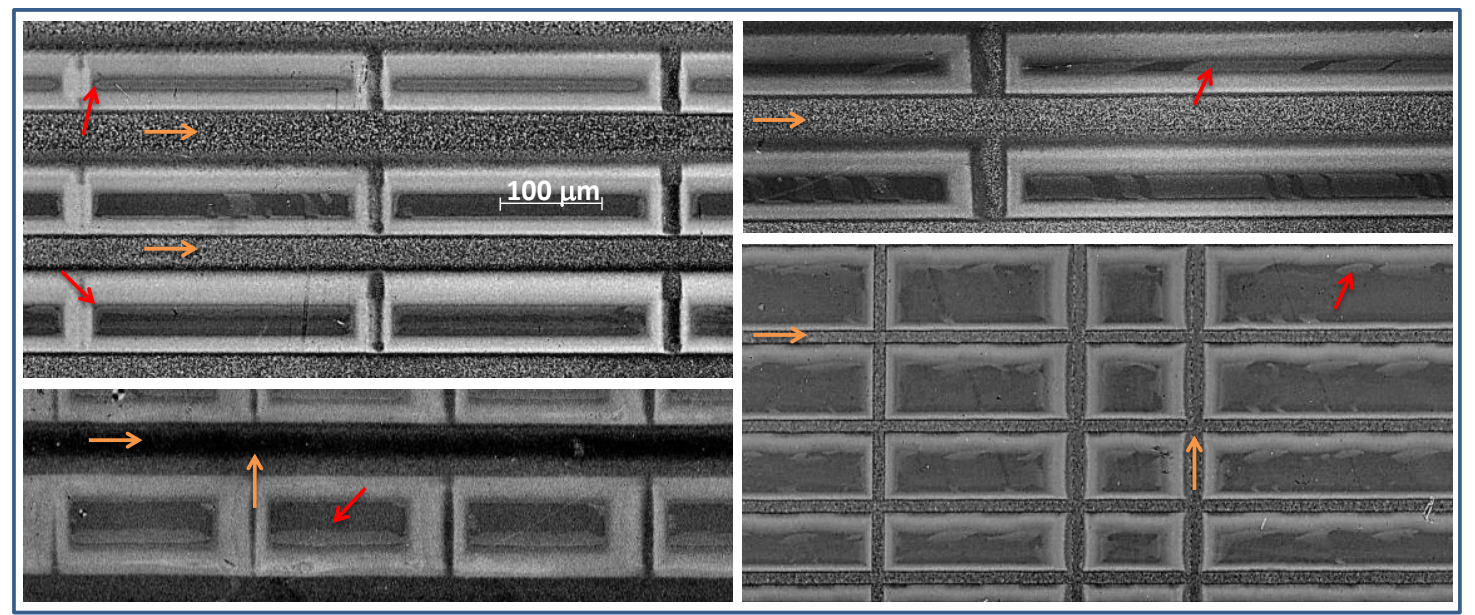

Figure 2.23 | Different shapes of magnetic domains. Orange arrows show where the stripes are written. Red arrows show the magnetic domains. 


\subsection{Pump-Probe Experiment}

Up to this point, we talked about measuring static magnetic properties of the samples. It is also possible to detect the magnetization dynamics of a sample. This can be done in a pump-probe experiment setup, which provides a way to probe a process with sub-picosecond resolution right after its initiation. In this setup, two laser beams (pump and probe) are focused on the sample. The pump beam interacts with the sample and triggers a process, which is monitored by a low-intensity probe beam at different delays with respect to the pump excitation.

We implemented a magneto-optical pump-probe setup in non-collinear configuration with different pump and probe wavelengths, to study the ultrafast processes in our samples (Figure 2.24). A femtosecond Ti:Saphire laser with 120 fs pulses at $800 \mathrm{~nm}$ wavelength was applied. The laser beam was split into two beams with high and low intensities (pump and probe) using a polarizing cube and a half-wave plate. Then, using a BBO (Beta Barium Borate) crystal, the probe wavelength was changed to 400 nm. The intensity of both beams was independently adjustable. The temporal delay between the two beams was introduced using a high-precision motorized stage. This delay stage added a variable distance for the probe beam to travel before reaching the sample. The stage controlled the relative pathway of the two beams and the delay time $\tau$

can be calculated as $\tau=\frac{2 x}{c}$, where $c$ is the speed of light and $x$ is the displacement of the mirrors on the stage. Pump and probe beams were focused on the sample, which was placed between two water-cooled coils. The sample could be shifted in three directions with a combination of three translation stages. A balanced photodiode connected to a lock-in amplifier detected the probe beam after the sample. The lock-in amplifier 
detected the signal at the chopper frequency on the pump beam path, fed from the output of the chopper controller.

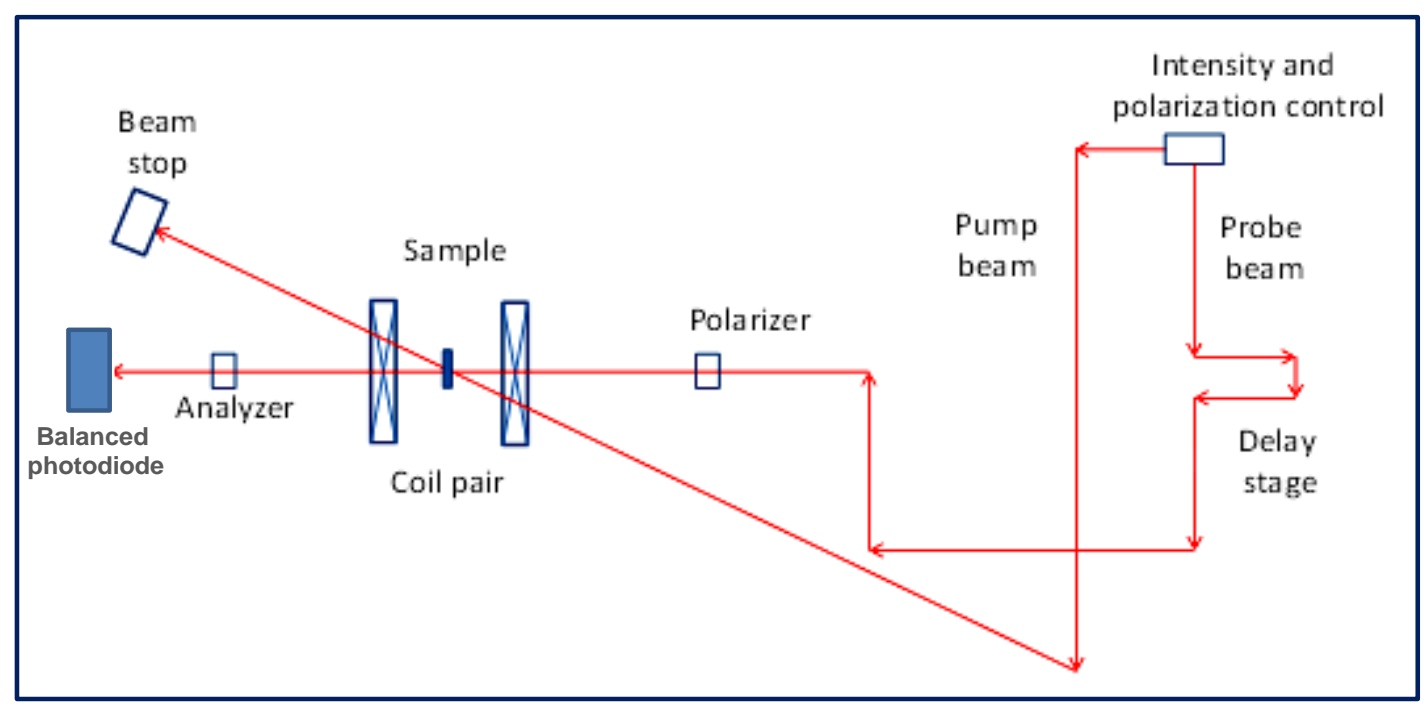

Figure 2.24 | Magneto-optical pump-probe setup.

To illustrate the general idea behind the pump-probe experiment, let us consider the example of magnetization precession triggered in a sample by the pump pulse (note that this is not what we did). Suppose we can detect the magnetization vector $\vec{M}$ of the sample (Figure 2.25). For this purpose, we need a light (probe) beam that reflects off to a detector. A probe pulse that arrives before the pump pulse detects $\vec{M}$ to be along $H_{\text {eff }}$. A new equilibrium condition and a new effective field $H_{\text {eff }}{ }^{\prime}$ would be created when a high intensity pump pulse is absorbed by the sample. The angle between $\vec{M}$ and $H_{\text {eff }}{ }^{\prime}$ leads to a torque on $\vec{M}$, initiating a precession. If we delay the probe pulse to reach the sample 50 ps after the pump pulse, then we would detect a magnetization oriented in a different direction (figure 2.25.b). Arriving $50 \mathrm{ps}$ after the pump pulse, requires the probe pulse to 
travel an additional $14 \mathrm{~mm}$. Provided precise alignment, the temporal resolution is equivalent to pulse duration at the sample (190 $f s$ ), or better, when fitting is used.

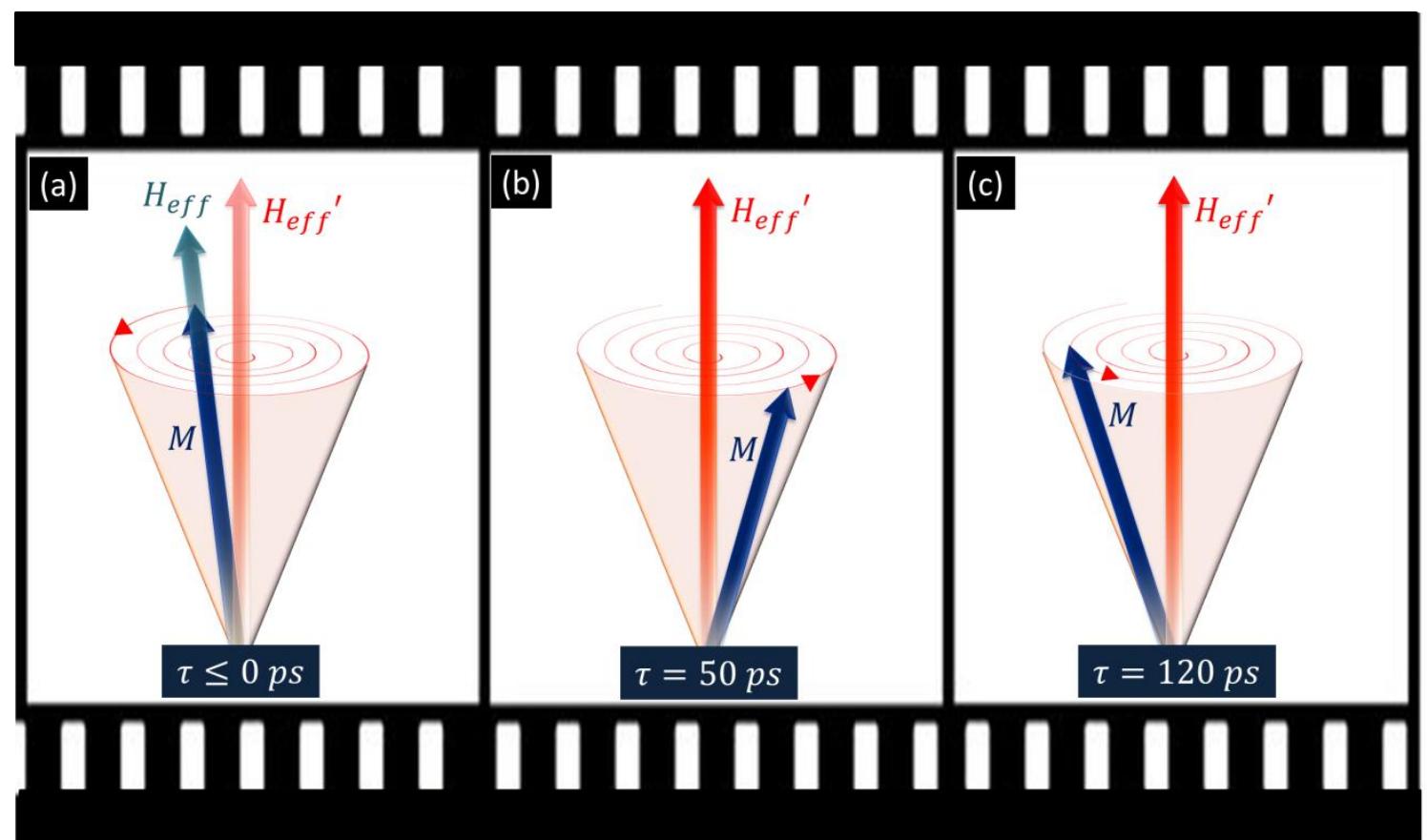

Figure 2.25 | An example to describe a pump-probe experiment. Assume a ferromagnetic sample with $\vec{M}$ along $H_{e f f}$ (a). A laser pulse (pump) is incident on the sample at $\tau=0$ leading to a new effective field $H_{e f f}{ }^{\prime}$, which causes $\vec{M}$ to start precessing. If the probe beam arrives at the sample before the pump it would detect $\vec{M}$ as it is in (a). A probe with delays 50 ps or 120 ps would result in cases (b) or (c). 50 and 120 ps delay times correspond to 14 and $36 \mathrm{~mm}$ respectively of extra distance that the probe pulse has to travel compared to the pump pulse.

We tested our time-resolved MOKE (TR-MOKE) setup on a Ni/Si(111) sample. An example measurement is shown in figure 2.26. The dip in the plot corresponds to the back-reflection echo of the strain wave in the film, which occurs 32 ps after the pump excitation. The experiment was done with no analyzer in the probe beam path. The stage was moved at $0.008 \mathrm{~mm} / \mathrm{s}$. 


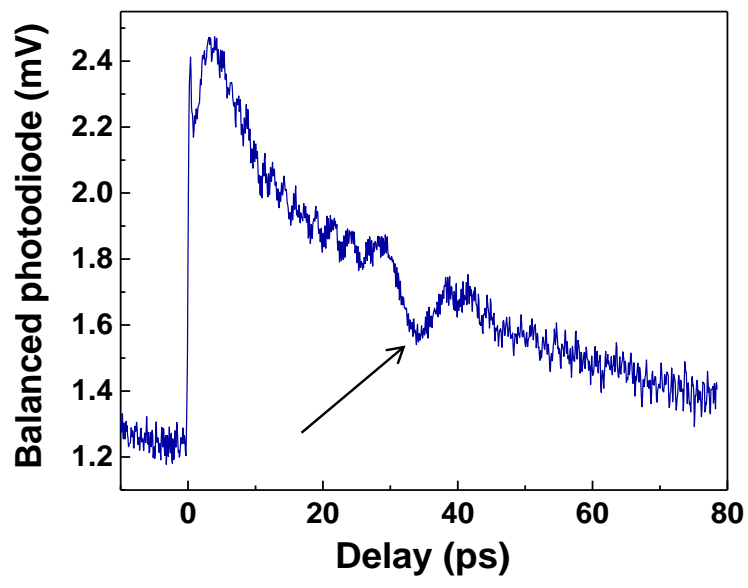

Figure 2.26 | Time-resolved MOKE measurement on Ni/Si sample.

In this work, we measured the magnetization dependence on delay time without triggering a precession. We detected UDM and the subsequent recovery of magnetization in the sample. Our setup geometry could be interchanged between MOKE (reflection) and Faraday (transmission) modes. For the thicker films, with weak transmission or films deposited on opaque substrates, we had to work in reflection mode. Figure 2.27 shows the signals obtained in reflection and transmission mode. The peak in the transmission mode comes from the temperature increase in the glass substrate. It decays within a characteristic time $\approx 700 \mathrm{fs}$ for the $\mathrm{Co} / \mathrm{Pd}$ sample used in this measurement. 


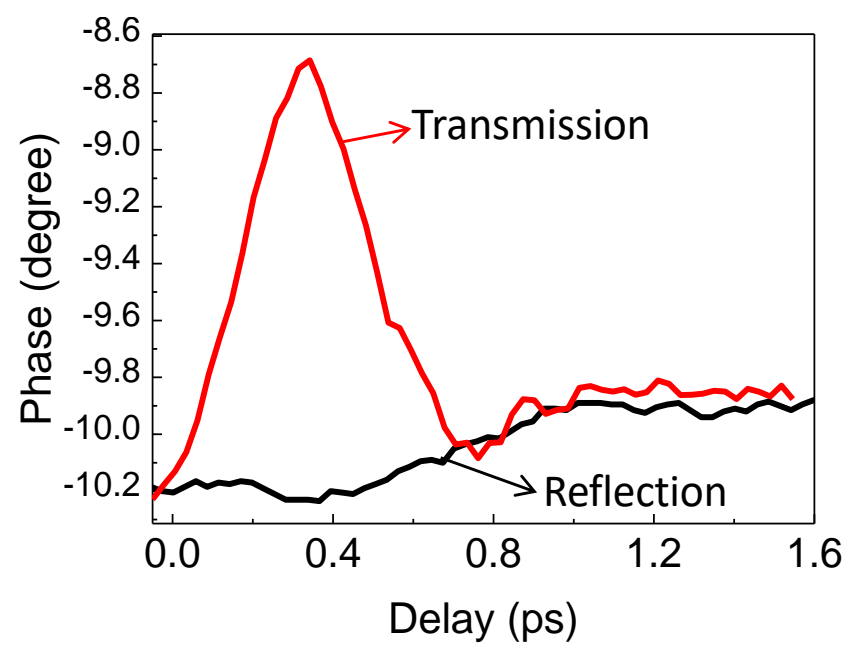

Figure 2.27 | Comparing pump-probe measurements in reflection and transmission geometries for a $\mathrm{Co} / \mathrm{Pd}$ sample on glass substrate.

A major difficulty in interpreting the results is finding a reliable method to separate magnetic and thermal contributions to the measurement. In magneto-optical pump-probe experiments the detected signal is proportional to the total rotation of polarization $\theta_{T}$, which can be written as the sum of a magnetic-induced rotation $\theta_{A}$ and a thermal contribution $\theta_{S}$ :

$$
\tilde{\theta}_{T}=\tilde{\theta}_{A}+\tilde{\theta}_{S}
$$

The tilde sign shows that $\theta$ is a complex value $\tilde{\theta}=\theta+i \varepsilon$, where $\varepsilon$ is the ellipticity and $\theta$ is the rotation of the beam polarization. The subscripts of $\theta$ denote whether the rotation is antisymmetric (A) or symmetric (S) with opposite applied fields.

To address this problem, we used a configuration known as crossed-polarization, where the polarizer and analyzer are almost crossed. Choosing analyzer angles away from crossing resulted in a symmetric peak or dip that corresponds to thermal effects (Figure 2.28). 


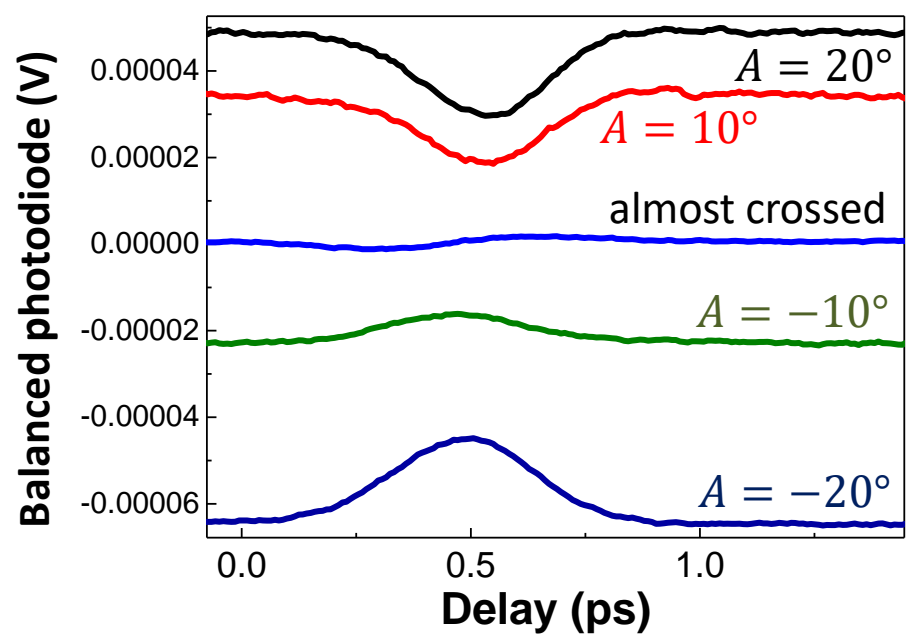

Figure 2.28 While thermal contributions were large, the magnetic signal was much smaller and harder to measure. To address this issue we kept the polarizer and analyzer almost at crossed angles (the middle curve), which minimizes the thermal contribution (symmetric peak or dip) to the signal.

In analyzing the data, we defined two variables; symmetric $S(\tau)$ and antisymmetric $A(\tau)$ in applied field. The symmetric part is obtained by adding the measurements at $\pm B$. The anti-symmetric one is calculated by subtracting the two. $S(\tau)$ and $A(\tau)$ correspond to thermal and magnetic effects, respectively. Figure 2.29 shows symmetric and anti-symmetric components measured at the correct analyzer angle, which resulted in distinct shapes for $S(\tau)$ and $A(\tau)$. The peak in the blue curve (symmetric part) corresponds to a sudden increase in temperature.

Using a high repetition-rate laser, means that we had to deal with heat building up in the sample, as there is not sufficient time for the heat to diffuse away between the pulses. This temperature increase led to a decreased magnetization. In addition, the applied energy per pulse was lower compared to other studies. These two features made it more difficult to detect UDM. 


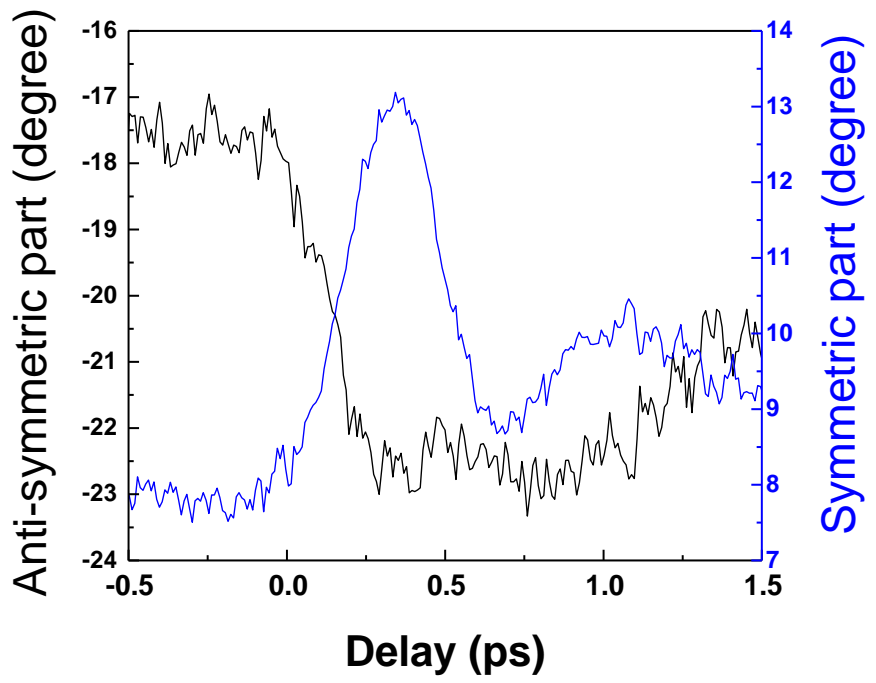

Figure 2.29 | Measurements at the correct analyzer angle results in distinct symmetric and anti-symmetric components, representing the thermal and magnetic parts of the signal, respectively. 


\section{CHAPTER 3: HEAT ACCUMULATION AND ALL-OPTICAL SWITCHING BY DOMAIN WALL MOTION IN CO/PD SUPERLATTICES ${ }^{1}$}

\subsection{Introduction}

In all-optical switching (AOS) of ferrimagnetic rare earth transition metal alloys the magnetization is reversed by 180 degrees [9]. This reversal was examined in detail and both polarization-independent and polarization-dependent switching have been reported $[15,16]$. As also observed with electron beam fields [17], the macrospin of the Landau-Lifshitz-Gilbert (LLG) equation fragments in the intense applied laser field. However, in rare-earth transition-metal alloys it reassembles in the opposite direction through an intermediate ferromagnetic state. More recently, AOS was observed in rareearth transition-metal multilayers [18, 19], ferromagnetic $\mathrm{Co} / \mathrm{Ir} / \mathrm{Ni} / \mathrm{Pt}$ heterostructures [19] and in ferromagnetic Co/Pt superlattices [20-22]. The observation of AOS in FePt granular materials [23], transparent YIG:Co [24], and in nanoscale domains [25] highlighted its potential for practical applications. The reversal has been investigated from different points of view with XMCD-PEEM [26, 27] and anomalous Hall Effect [28]. Skyrmions, which can be induced with focused laser beams [29], were found to form lattices in Gd-Fe multilayers [30].

\footnotetext{
${ }^{1}$ F. Hoveyda, E. Hohenstein, and S. Smadici, "Heat accumulation and all-optical switching by domain wall motion in Co/Pd superlattices," J. Phys.: Condens. Matter 29, 225801 (2017), doi.org/10.1088/1361$\underline{648 \mathrm{X} / \mathrm{aa} 6 \mathrm{c} 93}$
} 
Models of magnetization ultrafast time dependence in ferrimagnets apply the Landau-Lifshitz-Bloch (LLB) equations accounting for the variation in macrospin magnitude [31] or microscopic atomistic calculations with exchange interactions [32-34], relying on specific microscopic exchange interactions. For instance, for a ferrimagnet with two $(A$ and $B)$ sub-lattices, Heisenberg exchange interactions $J_{A A}, J_{A B}$, and $J_{B B}$ are considered. Little of this applies to a ferromagnet, which has only one interaction constant $J$, leaving open the question of the origin of AOS in these materials. Models may include the Inverse Faraday Effect transient magnetic field induced by the laser pulse in the material [35-37], while others do not [38, 39].

Previous experiments on ferrimagnetic materials were done with single-pulse excitation. The relatively low repetition rate $(\mathrm{kHz}$ range) lasers used in these studies allowed sufficient movement of the sample between pulses, so that pulses did not overlap over the same sample area. This is important in ferrimagnets because each pulse initiates a reversal [32]. The AOS in ferromagnetic materials also utilized a low-repetition rate laser. However, AOS was observed when the pulses overlapped on the sample [19-22]. This suggested that it may be possible to initiate magnetization reversal in ferromagnets by directly applying the faster sequence of pulses from a TiS oscillator. In this work, we scanned an ultrafast $80 \mathrm{MHz}$ repetition rate laser field on $\mathrm{Co} / \mathrm{Pd}$ superlattices. Polarization-independent AOS was observed. In our experimental conditions, reversal by domain wall (DW) motion becomes visible after a few ms. Polarizing and magnetic microscopy images show a reversal driven by heat accumulation in the sample and inplane thermal gradients. 


\subsection{Experiments}

\subsubsection{Samples and experimental setups}

The $[\mathrm{Co} / \mathrm{Pd}]$ multilayer samples $\mathrm{A}$ and $\mathrm{B}$ were grown by e-beam evaporation at room temperature. Before the deposition, the Corning white-water glass substrates were immersed in Nanostrip solution for five minutes, placed in acetone and then methanol, and sonicated in each liquid for $10 \mathrm{~min}$ at $60{ }^{\circ} \mathrm{C}$. The deposition was carried out at $2 \times 10^{-6}$ Torr pressure with the substrate at an angle of $45 \circ$ to the incident beam. The substrate rotated around its normal at $5 \mathrm{RPM}$ during deposition. The target multilayer structure was $4 \times[\mathrm{Co} / \mathrm{Pd}]$ with $0.7 \mathrm{~nm}$ thick Co and $1 \mathrm{~nm}$ thick Pd layers. The total thicknesses measured with AFM were $4.1 \mathrm{~nm}$ and $6.2 \mathrm{~nm}$ for samples A and B. Sample A is thinner because it was placed further out from the crucibles during deposition.

MOKE measurements were performed in both longitudinal and polar geometry. The variable-temperature setup consisted of a HeNe laser, rotatable Glan-Thompson polarizer and analyzer, photoelastic modulator (PEM), an electromagnet (GMW) driven by a bipolar power supply (KEPCO BOP 50-8ML), and a photodiode detector connected to a lock-in amplifier. The sample was mounted on an Al holder and placed at the center of the electromagnet. The incident beam was p-polarized and was focused on the sample. The incidence angle in the L-MOKE geometry was $25 \mathrm{deg}$. The reflected beam from sample surface passed through the PEM (Hinds PEM-90) and analyzer. Hysteresis loops in the P- and L-MOKE geometries were obtained by averaging the results from running a sixteen-cycle waveform (figure 1(a)). 

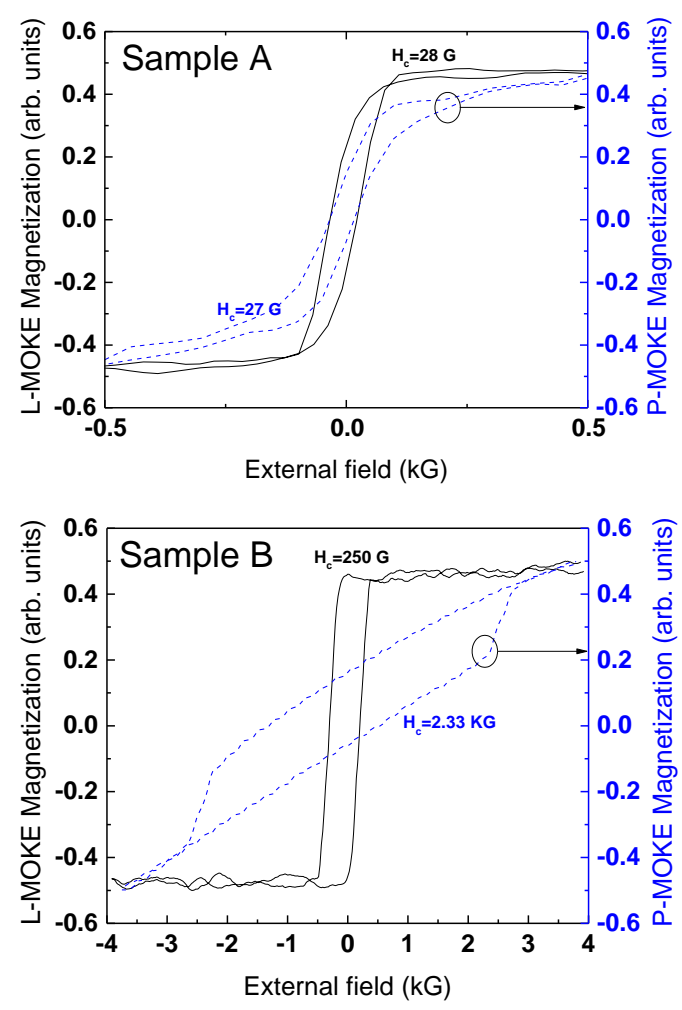

a

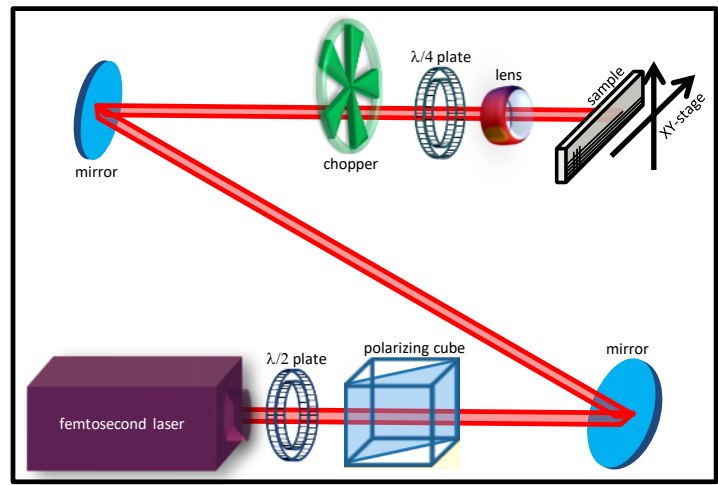

b

Figure 3.1 | (a) L- and P-MOKE hysteresis loops at room temperature for samples A and B. (b) Sketch of the writing setup.

The fluence of an ultrafast TiS laser with 120 fs pulses at $800 \mathrm{~nm}$ wavelength and a repetition rate of $80 \mathrm{MHz}$ was adjusted by a combination of a polarizing cube and a half-wave plate (HWP) attached to a motorized rotation stage (figure 1(b)). A quarterwave plate (QWP) was applied to modify the beam polarization. The laser beam was focused to a typical size of $25 \mu \mathrm{m}$. The sample was AC demagnetized before the writing, 
to increase the number of magnetic domains, and was scanned along the two directions orthogonal to the beam using two motorized stages. The writing speed $\mathrm{v}_{\mathrm{s}}$ was varied between $0.1 \mathrm{~mm} / \mathrm{s}$ and $10 \mathrm{~mm} / \mathrm{s}$. A small beam asymmetry could be removed by placing a blade before the lens to partially cut the beam.

The sample response to TiS laser scans was examined with polarizing and magnetic force microscopy (MFM). Polarizing microscopy imaging in transmission mode was carried out with a Zeiss Imager microscope. The polarizer and rotatable analyzer were crossed, and then offset a small angle in both directions. The images were then subtracted to enhance the birefringent contrast. Images were also made with the polarizer and analyzer removed.

Magnetic domains were imaged using an Asylum Research MFM (MFP-3D-BIO) with a low moment tip from Nanosensors (SSS-MFMR). It was operated in tapping mode at a lift height of $50 \mathrm{~nm}$. The tip was magnetized before imaging with a permanent magnet attached to a moving stage.

\subsubsection{Results}

Polarizing microscopy images made before writing show irregular angular magnetic domains (figure 2(a)). Patterns made during the writing procedure appear as 'stripes' in the images. A birefringent contrast at the stripe center is visible after writing (figure 2(b)). For clarity, an area in figure 2(b) was selected with background domains induced by AC demagnetization that are larger than the field of view (unlike the domains in figure 2(a)). The direction of magnetization after scanning is opposite to the direction of magnetization in these background domains. This contrast is magnetic, not structural, because it matches the background magnetic domain contrast and angular shape. The 
contrast is not changed with azimuthal sample rotation, pointing to reversed magnetic domains with magnetization oriented perpendicular to the surface. Reversals with the magnetization pointing down on a magnetization-up background were also observed (not shown).

Further increases in fluence give a thermal demagnetization speckle pattern at stripe center. Regular thermal demagnetization patterns have been obtained before in ferromagnetic $\mathrm{Co} / \mathrm{Pd}$ superlattices with 12 ns pulses [40]. The low-fluence features are no longer visible in the thermally demagnetized regions, unlike what would be expected of structural changes, further supporting their magnetic origin.

Two types of AOS have so far been observed in ferromagnetic materials: singleand multiple-pulse ('cumulative') switching [41]. At a typical writing speed $\mathrm{v}_{\mathrm{s}}=5 \mathrm{~mm} / \mathrm{s}$ and beam diameter of $25 \mu \mathrm{m}, 4 \times 10^{5}$ consecutive pulses are incident on the sample before the beam moves away. Therefore, the observed AOS in Co/Pd is cumulative. Similar results are obtained with right- (RCP), left- (LCP) and linearlypolarized (LP) light, supporting a thermal model for cumulative switching in $\mathrm{Co} / \mathrm{Pd}$ superlattices. This is in contrast to cumulative switching in $\mathrm{Co} / \mathrm{Pt}$ superlattices, in which the magnetization was reversed with RCP or LCP light and absent with LP light [18]. In addition, different results are obtained with the same pulse power and different scanning speeds, further supporting a thermal model. 

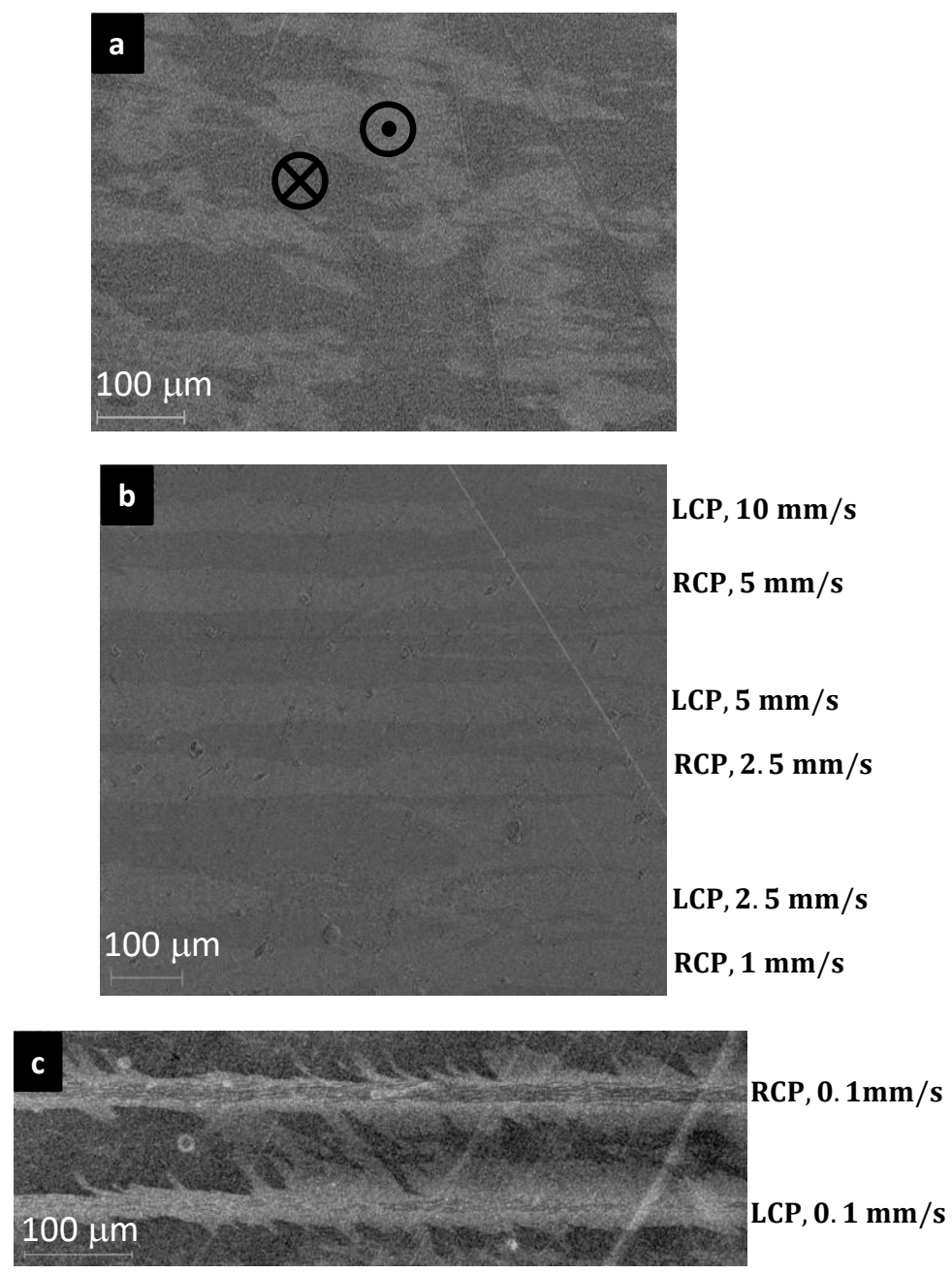

Figure 3.2 | (a) Background domains of sample A. (b) AOS in sample A at $150 \mathrm{~mW}$ power. (c) Nucleation of secondary domains at stripe center in sample A at $125 \mathrm{~mW}$ power and smaller speed.

Observations also point toward the relevance of long-range processes. Images were made after writing with beams of higher fluence. Fluence decreases gradually toward the wings of the beam profile, from a maximum at the center (figure 3(a), inset). The same sequence of final states is expected across one stripe, as in images of a stripe center made at different powers. Similar results were observed. For instance, the narrowest magnetic domains near the stripes in figure 3(a) are reversed regions, corresponding to regions at the center of the stripe in figure 2. However, for widely- 
spaced stripes, images also show longer range domains, extending far away from areas exposed to the beam (figure 3(a), top part). Complementary domain contrast is also observed for closely-spaced stripes (figure 3(a), lower part) and for domains on opposite sides of one stripe (figure 3(b)). This suggests that the long-range demagnetizing fields should be considered.
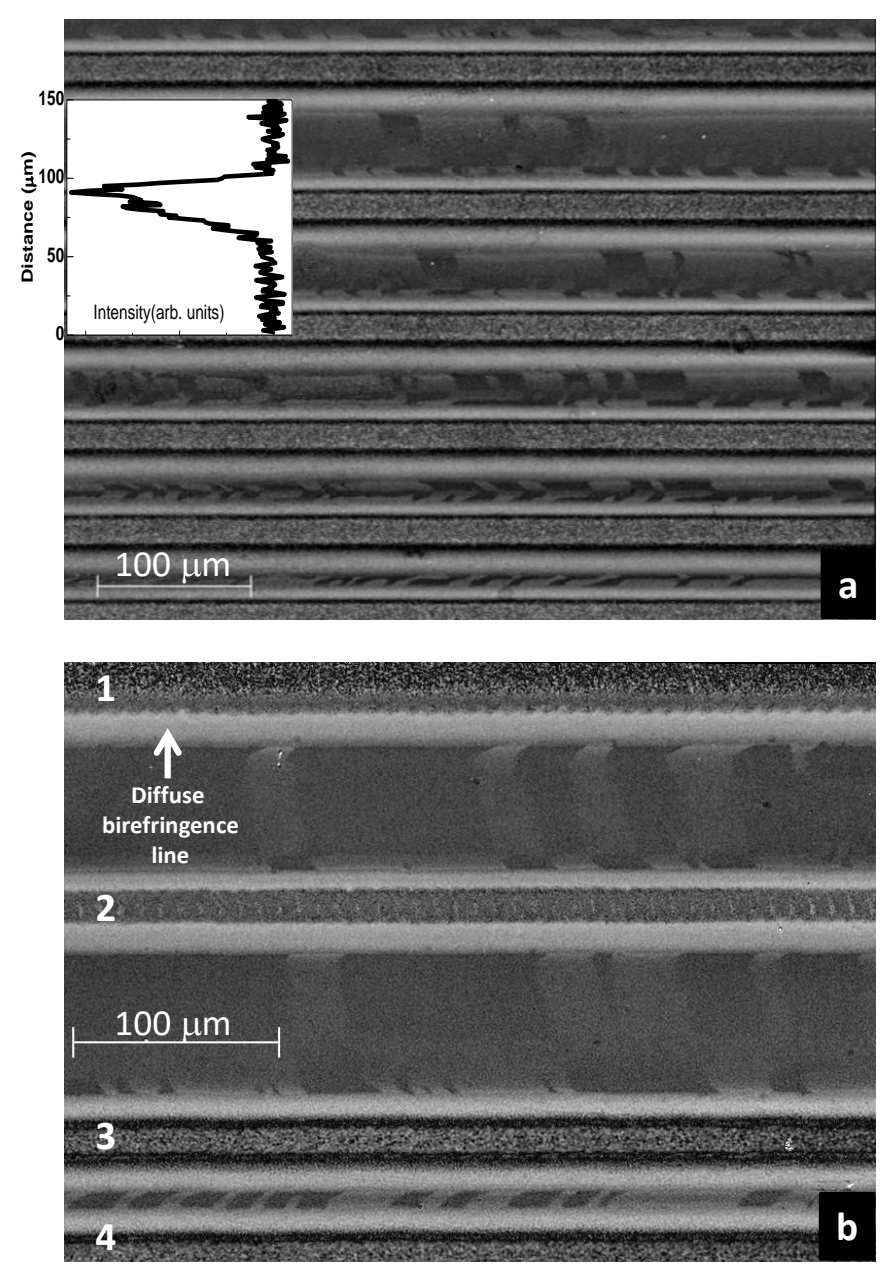

Figure 3.3 | Polarizing microscopy images of sample B. (a) AOS domains extend away from under the laser beam profile (top) and show complementary contrast at small stripe spacing (bottom). All stripes were written with $600 \mathrm{~mW}$ power, LP light, and at $10 \mathrm{~mm} \mathrm{~s}^{-1}$ speed. (b) Opposite reversal across a stripe. Writing parameters are $(380 \mathrm{~mW}, \mathrm{LCP}, 1 \mathrm{~mm}$ $\left.\mathrm{s}^{-1}\right)$ for 1, $\left(220 \mathrm{~mW}, \mathrm{LCP}, 1 \mathrm{~mm} \mathrm{~s}^{-1}\right)$ for 2, $\left(600 \mathrm{~mW}, \mathrm{LP}, 10 \mathrm{~mm} \mathrm{~s}^{-1}\right)$ for 3 and 4. 
MFM measurements gave further insight into the magnetic structure. Small domains are oriented with magnetization perpendicular to the surface at stripe center (figure 4), consistent with previous results [40]. Domain walls were observed toward the edges, of the same shape as the domain walls in polarizing microscopy. This shows that the magnetic structure is not uniform within the sample (figure 4, insets), with surface magnetic closure domains forming to minimize the demagnetizing field (internal magnetostatic) energy. In our case, the top layer in-plane magnetization is probed in MFM, but not in polarizing microscopy at normal incidence, while a buried layer out-ofplane magnetization, not visible in MFM, appears in transmission polarizing microscopy. The orientation of the domain walls (DW) in the immediate vicinity of the stripe makes an 'arrow' pointing in the direction of the laser spot motion on the sample (figure 5). This suggests that DW processes should be considered in a reversal model. DW may change direction further away from the stripe as in figure 3(b), from a residual in-plane magnetic anisotropy induced during deposition.

A diffuse bright birefringent contrast near the thermal demagnetization areas, without the angular edges of magnetic domains, is also visible in polarizing microscopy images (figure 3). Unlike the magnetic domains, this birefringence contrast changes sign with sample rotation with a period consistent with structural birefringence of the glass substrate [42, 43] and small linear defects induced by the laser beam [44, 45]. This feature is absent in MFM images (figure 4(a)), but present in the polarizing microscopy image (figure 4(b)), also consistent with a substrate location. 

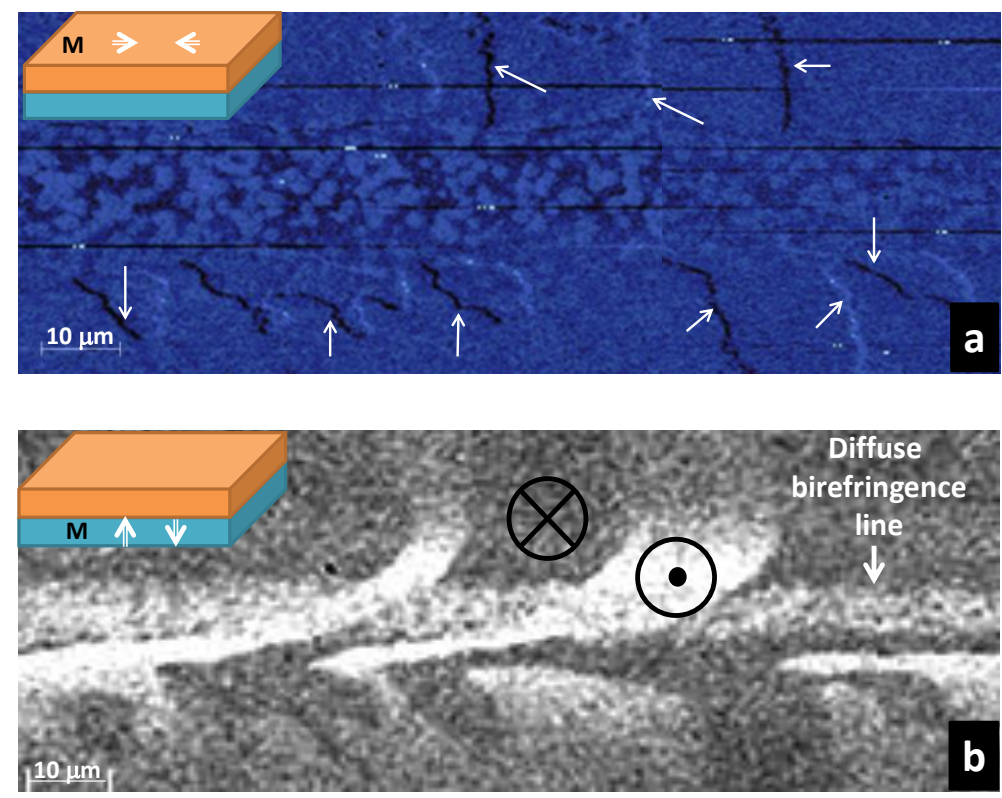

Figure 3.4 | MFM (a) and polarizing microscopy (b) images of a stripe in sample B (100 $\mathrm{mW}, \mathrm{LP}, 10 \mathrm{~mm} \mathrm{~s}^{-1}$ ). The arrows show the domain walls. Inset: sketch of a side view of the magnetic structure.

The importance of DW points to an expanded timescale of the switching process. To investigate this more directly, measurements were made with a chopped beam and moving samples. This resulted in sequences of reversed 'dots', each corresponding to the time intervals during which the chopper blade did not block the beam (figure 6). Domains show a filamentary structure in polarizing microscopy images, as they grow from under the laser beam. The front-back asymmetry, with domains offset in the scanning direction with respect to the diffuse background, shows a delay on the order of $\sim \frac{25 \mu \mathrm{m}}{5 \mathrm{~mm} / \mathrm{s}}=5 \mathrm{~ms}$ in the initiation of magnetization switching. Therefore, a slow process is part of the complete reversal. Fully developing AOS in Co/Pd takes significant time. 


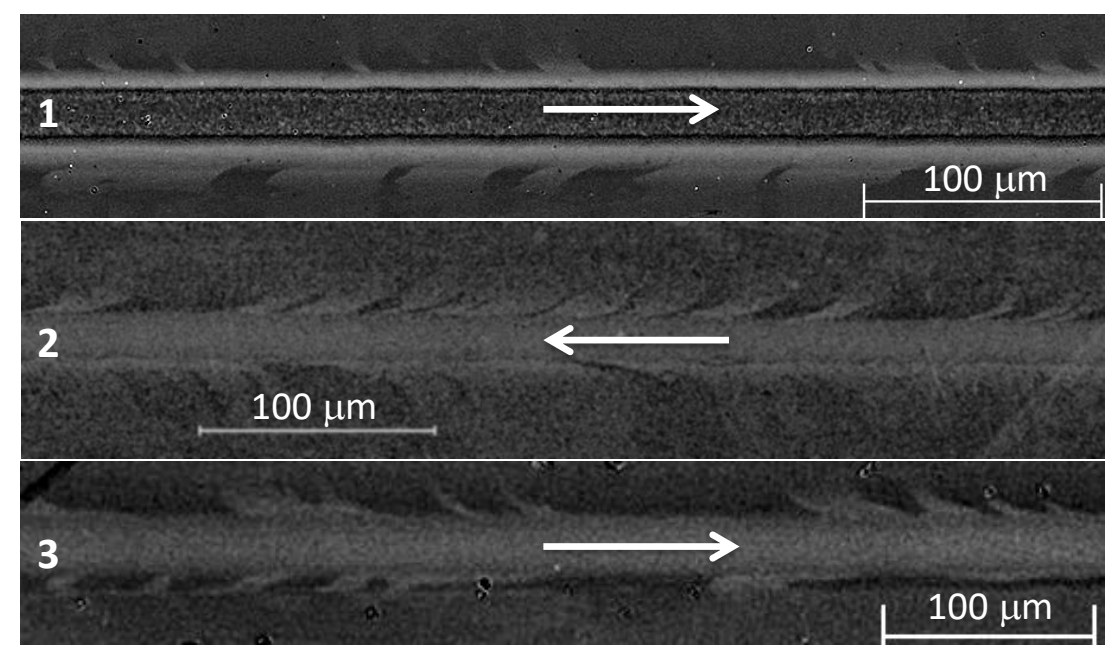

Figure 3.5 | Domain walls are oriented differently, depending on the scan direction. The arrows show the direction of laser beam motion. Writing parameters for stripe 1, 2, and 3 were (sample B, $600 \mathrm{~mW}$, LCP, $10 \mathrm{~mm} \mathrm{~s}^{-1}$ ), (sample A, $125 \mathrm{~mW}, \mathrm{RCP}, 10 \mathrm{~mm} \mathrm{~s}^{-1}$ ), and (sample A, $190 \mathrm{~mW}, \mathrm{RCP}, 10 \mathrm{~mm} \mathrm{~s}^{-1}$ ), respectively.

\subsection{Discussion}

The high-repetition rate laser requires a reconsideration of the assumptions made in previous experiments with low-repetition rate sources. Stronger thermal effects are expected compared to previous AOS experiments.

The long timescale filamentary DW structure in dot images supports a reversal by DW motion in our case, with magnetization rotation at the DW location. Models of ultrafast reversal with uniform magnetization rotation are replaced with a model of reversal by DW motion.

\subsubsection{Heat accumulation and in-plane heat diffusion}

The laser outputs a sequence of pulses. At the shortest timescale (up to a few ps after the pulse), the sharp transient increase in temperature $\mathrm{T}_{\mathrm{tr}}(\mathrm{t})$ of the lattice is illustrated by the red peaks in the sketch in the top panel of figure 6 , where the $12.5 \mathrm{~ns}$ 
spacing between the peaks has been greatly increased for clarity. There are also transient electron $T_{e}$ and $\operatorname{spin} T_{s}$ temperatures in three-temperature (3T) models of magnetic materials [33-35][46-48]. $T_{e}$ and $T_{s}$ have strong variations over a few ps, after which all three temperatures are the same $T=T_{p}=T_{s}=T_{e}$, gradually decreasing toward the initial temperature. In-plane heat diffusion can be neglected at these timescales, with the models becoming effectively one-dimensional.

At the intermediate timescale, in-plane heat diffusion, which is relatively slow and can be safely neglected in the shortest range, should be included. The heat diffusion equation for a material isotropic in the surface plane is

$$
\rho c \frac{\partial T}{\partial t}=K_{\|}\left(\frac{\partial^{2} T}{\partial x^{2}}+\frac{\partial^{2} T}{\partial y^{2}}\right)+K_{z}\left(\frac{\partial^{2} T}{\partial z^{2}}\right)
$$

where $\rho, c, K_{/,}, K_{z}$ are the density, the specific heat, in-plane and along $z$-axis thermal conductivities of the material. Heat diffusion occurs initially mostly in the film $\left(K_{/ /} \gg K_{z}\right)$.

Even with the large diffusivity $D=\frac{K}{\rho c}$ of bulk metals, it would take $t \sim \frac{d^{2}}{D} \sim \frac{(30 \mu \mathrm{m})^{2}}{0.1 \mathrm{~cm}^{2} / \mathrm{s}} \sim 0.1 \mathrm{~ms} \gg t_{\text {rep }}=12.5 \mathrm{~ns}=\frac{1}{v_{\text {rep }}}$ for heat to diffuse over a distance equal to the beam diameter. The high repetition rate leaves too little time for the sample to cool to the initial temperature after each pulse and heat accumulation between pulses cannot be neglected [49]. However, the film does not cool very effectively because it is very thin. Therefore, at the longest timescale heat diffusion into the substrate becomes increasingly more important, making up for a reduced $K_{z}$ with a larger heat transfer area. Although the film is the primary factor in absorbing the light and heating the sample, the time evolution of the temperature during cooling is determined mainly by the substrate. 
These processes combine to give a gradual heat accumulation and rise in sample temperature. The solution $T(t)$ is known in closed form for a uniform beam profile [50]. For a stationary beam with a Gaussian profile, the heat accumulation and cooling can be directly illustrated when the small sideways heat transfer in the film is neglected. The time dependence of the temperature at the surface of a semi-infinite medium is obtained by solving the heat diffusion equation, following excitation by a sequence of $\delta$ - function pulses, periodically interrupted by the chopper. It is advantageous to write the temperature as a sum over different frequency components

$$
\begin{aligned}
& T(t, r)=\operatorname{Re} \sum_{l=0}^{\infty}\left[\frac{\pi}{4} T_{0}\left(t, r, l \omega_{r e p}\right)+\sum_{m=0}^{\infty} \frac{1}{2 i(2 m+1)} \times\left(T _ { 0 } \left(t, r, l \omega_{r e p}+\right.\right.\right. \\
& \left.\left.\left.(2 m+1) \omega_{c h}\right)-T_{0}\left(t, r, l \omega_{r e p}-(2 m+1) \omega_{c h}\right)\right)\right]
\end{aligned}
$$

where the sums over $l$ and $m$ are over the femtosecond comb and the chopper squarewave spectrum, and solve the heat diffusion equation at each frequency separately. Then, $T_{0}(t, r, \omega)=\int_{0}^{\infty} d k k J_{0}(k r) \frac{1}{\sqrt{k^{2}+\frac{i \omega}{D}}} e^{-\frac{k^{2} \omega_{0}^{2}}{8}} e^{i \omega t}$, where $\omega_{0}$ is the beam diameter and $D$ is the heat diffusion constant. The temperature $T(t, r=0)$ at the center of the beam is plotted in figure 6 and shows the gradual heat accumulation $\mathrm{T}_{\mathrm{acc}}(\mathrm{t})$ (green dashed line in the top panel) for $\frac{D T_{c h}}{\omega_{0}^{2}}=0.04$, where $T_{\text {ch }}$ is the chopping period. For clarity $\frac{T_{c h}}{T_{r e p}}=$ 10 has been chosen, or 10 pulses incident on the sample during the chopping period (in practice this number is $\sim 10^{5}$ ). 

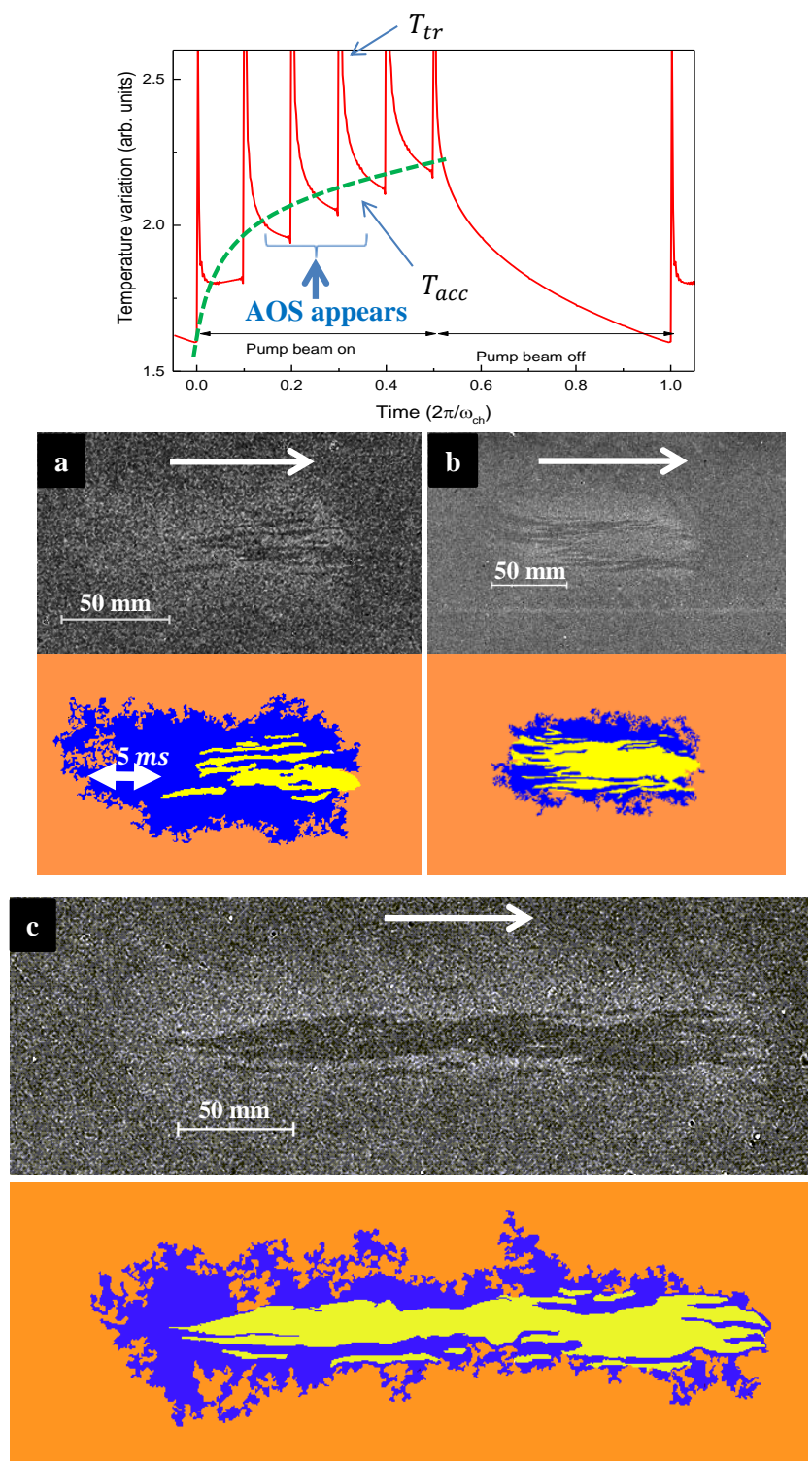

Figure 3.6 | Magnetic dots made with chopped beams show a delay in the onset of reversal. These stripes were written in sample A. Writing parameters for (a)-(c) were $\left(\mathrm{f}=20 \mathrm{~Hz}, 230 \mathrm{~mW}, \mathrm{LCP}, 5 \mathrm{~mm} \mathrm{~s}^{-1}\right),\left(\mathrm{f}=10 \mathrm{~Hz}, 230 \mathrm{~mW}, \mathrm{LCP}, 2.5 \mathrm{~mm} \mathrm{~s}^{-1}\right)$ and $(\mathrm{f}=10 \mathrm{~Hz}$, $230 \mathrm{~mW}, \mathrm{RCP}, 10 \mathrm{~mm} \mathrm{~s}^{-1}$ ), respectively, where $\mathrm{f}$ is the chopping frequency. The arrows show the direction of laser beam motion. The top panel shows the heat accumulation at the center of a Gaussian beam. 
This time-dependence is being modified when a neighboring point on the sample is considered because the attenuation length from the heat diffusion equation $\lambda_{\text {att }}=\sqrt{2 D / \omega}$ depends on $\omega$. The $T_{\text {tr }}$ spikes, with a large $\omega$, are damped out in $<1 \mu \mathrm{m}$. However, the more slowly-changing $T_{\text {acc }}$ extends over a considerable distance ( $\sim 250 \mu \mathrm{m}$ in the film and $\sim 25 \mu \mathrm{m}$ in glass). With time, heat diffusion appears as a strongly-damped 'wave' with large thermal gradients $\partial \mathrm{T} / \partial \mathrm{r}$ along the surface at locations away from the initial disturbance.

A quantitative analysis of heat accumulation and diffusion and a solution for $T_{a c c}$ $(x, y, z, t)$ when the beam is moved on the sample is a more complicated problem because the time it takes a moving beam to traverse a distance equal to its diameter $t \sim \frac{25 \mu \mathrm{m}}{10 \mathrm{~mm} / \mathrm{s}}=$ $2.5 \mathrm{~ms}$ at typical scanning speeds $v_{s}=10 \mathrm{~mm} / \mathrm{s}$ is comparable to the heat diffusion time. A numerical solution of the heat diffusion equation for moving beams is required and is presented in the next section.

\subsubsection{Domain wall dynamics}

The gradual heat accumulation explains the delay in the onset of AOS in figure 6. The chopper turns the pump laser beam on for half of the chopping period. During the 'on' interval, the sequence of pulses gradually increases the temperature $T_{\text {acc }}$, shown by the dashed green curve. If we consider a stationary beam first, the sample partly cools during the 'off' interval, and the process is repeated, with the temperature rising until a dynamic equilibrium is obtained between a periodic heating and cooling of the sample, modulated at the chopper frequency. This temperature modulation has been confirmed in our samples in separate pump-probe experiments (in preparation). When we scan the 
sample under the chopped beam, areas exposed first will have a smaller temperature increase because the beam immediately moves away. In contrast, later areas will receive a larger number of pulses as the beam sweeps across them. This explains the AOS delay in figure 6, when energy has to be deposited first in the structure during the first few ms for domains to become visible, either because AOS is not initiated or because DW do not move well unless $T_{\text {acc }}$ is sufficiently large.

The variation in the nucleation patterns in $\mathrm{Co} / \mathrm{Pd}$ is similar to the stochastic nucleation of reversed domains in all-optical switching of ferromagnetic $\mathrm{Co} / \mathrm{Pt}$ [22]. Nucleation may take a few ms, corresponding to several tens of thousands of pulses in our case, but only 60 pulses when a $1 \mathrm{~Hz}$ repetition rate is applied to Co/Pt [22]. The reversal is described with two characteristic domain growth times in the $\mathrm{Co} / \mathrm{Pt}$ experiment, which has very small heat accumulation [22], in contrast to our case.

DW motion at increased temperatures induced by laser fields has been investigated extensively in magnetic bubble materials. The energy of a cylindrical magnetic domain of radius $r$ and thickness $h$ with magnetization $M_{s}$ perpendicular to the surface in the limit $\mathrm{r} \gg \mathrm{h}$ is

$$
\mathrm{E}=2 \pi r h \sigma_{\omega}+4 \pi r h^{2} M_{S}^{2}-8 \pi r h^{2} M_{S}^{2} \ln \left(\frac{8 r}{h}\right)+2 \pi r^{2} h M_{S} H
$$

where $\sigma_{\omega}$ is the domain wall energy and $H$ is the applied external magnetic field. The first term is the domain wall energy, the second and third are the demagnetizing field energy. The DW equilibrium condition can be expressed as a balance of three forces which, when $\sigma_{\omega}$ and $M_{s}$ are constant, are given by the derivatives of the corresponding energy terms: $F_{\omega}=-2 \pi r h \sigma_{\omega}$ for the domain wall energy, $F_{D}=4 \pi h^{2} M_{s}^{2}(1+$ 
$\left.2 \ln \left(\frac{8 r}{h}\right)\right)$ for the demagnetizing field energy and $F_{H}=-4 \pi r h M_{S} H$ for the external field energy [51-53]. $F_{\omega}$ tends to decrease the domain size, while $F_{D}$ tends to increase it. The direction of $F_{H}$ depends on the direction of the external field. The condition $F_{\omega}+F_{D}+$ $F_{H}=0$ can give a stable solution, if the external field $H$ is not too large, which has been the subject of extensive investigations in magnetic bubble materials, for instance in iron garnet $[54,55]$.

We apply the same approach to explain our observations. In contrast to experiments in magnetic bubble materials, no external field $H$ is applied in our case (the light magnetic field is negligible at our fluence) and $F_{H}=0$. The superlattices are also much thinner, to obtain the required perpendicular magnetic anisotropy. However, the domain wall and demagnetizing field energy remain comparable because $M_{s}$ is larger than in magnetic bubble materials, as has been confirmed for $\mathrm{Co} / \mathrm{Pt}$ [21]. The importance of demagnetizing field energy is further supported by the observed surface magnetic closure domains. The equation $F_{\omega}+F_{D}=0$ has one solution, which corresponds to the unstable solution of magnetic bubble materials (the stable solution is at infinite radius). It is unstable because increasing $r$ slightly, increases the outward force $F_{D}$, further pushing the DW to larger values. Similarly, decreasing $r$ slightly reduces $F_{D}$ and the domain collapses. In practice, this solution is stabilized because, to move a DW in a sample with a finite coercive field $H_{\mathfrak{c}}$, it is necessary to supply energy to compensate for energy dissipation associated with DW motion. This gives in the steady-state ( $v=$ const.)

$$
v=\frac{\eta}{2 M_{s}}\left(\frac{F}{2 \pi r h}\right)-\eta H_{c}
$$


where the case of domain expansion is considered $(v>0), \eta$ is the DW mobility and the net force $F$ has been divided by the DW area [53-56]. The last term changes sign when the domain contracts $(v<0)$.

The force that overcomes the coercive field term in the velocity equation above and pushes the DW from under the laser beam comes from heat accumulation $\left(F_{u}\right.$, corresponding to time variations of a spatially uniform temperature $T$ ) and temperature in-plane gradients $\left(F_{g}\right)$. The force $F u$ for temperature changes from $T$ to $\mathrm{T}^{\prime}$ is

$$
\frac{F_{u}}{2 \pi r h}=\frac{\Delta\left(F_{\omega}+F_{D}\right)}{2 \pi r h}=-\frac{\Delta \sigma_{\omega}}{r}+\frac{4 h M_{S}}{r}\left(1+2 \ln \left(\frac{8 r}{h}\right)\right) \Delta M_{S}
$$

Where $\Delta \sigma_{\omega}=\sigma_{\omega}\left(T^{\prime}\right)-\sigma_{\omega}(T)$ and $\Delta M_{s}=M_{s}\left(T^{\prime}\right)-M_{s}(T)$. For instance, if $\sigma_{\omega}$ decreases faster with $T$ than $M_{s}^{2}$ the wall energy is relatively smaller at higher temperatures, $F_{u}$ points outward and the domain expands at high $T$. The opposite situation can also occur [57]. In addition, temperature-induced spatial gradients in $\sigma_{\omega}$ and $M_{s}$ and therefore in the energy $E$ give a force $F_{g}$ equal to [52]

$$
\frac{F_{g}}{2 \pi r h}=-\nabla \sigma_{\omega}+4 h M_{s}\left(-1+2 \ln \left(\frac{8 r}{h}\right)\right) \nabla M_{S}
$$

where $\nabla \sigma_{\omega}=\frac{\partial \sigma_{\omega}}{\partial T} \nabla T$ and $\nabla M_{s}=\frac{\partial M_{s}}{\partial T} \nabla T$ are in-plane gradients of DW energy and magnetization induced by an in-plane temperature gradient $\nabla \mathrm{T}$. A positive sign of $F_{g}$ corresponds to a force pointing outward in a gradient $\nabla \mathrm{T}<0$ (a reduction of $T$ when moving away from the center of the beam). A positive first term requires $\frac{\partial \sigma_{\omega}}{\partial T}>0$, which, although not the usual situation, has been observed in SmxTb1-xFeO3 [44][57]. The second term in $F_{g}$ is positive for $\nabla \mathrm{T}<0$ and $\frac{\partial M_{S}}{\partial T}<0$ except at very small $r$. 
Numerical solutions of the heat diffusion equation are necessary to obtain the temporal and spatial dependence of temperature for a moving beam. As before, we neglect the small sideways heat transfer in the thin metallic film. One frame of the results is shown in figure 7 (the full movie is in the supplementary information. $F_{u}$ dominates at the center of the beam, where the gradients are small and temperatures the highest and, when the conditions presented above are satisfied, pushes the DW from under the beam (figure 6). In addition, the heat front gives a force $F_{g}$ lined up with the temperature gradient (equation (6)) and at an angle to the scanning direction. As the temperature decreases and $H_{\mathrm{c}}$ increases, this transient heat wave front leaves its imprint in the magnetic structures observed with polarizing microscopy (figure 5). In contrast, calculations for substrates with $10 \times \mathrm{D}_{\text {glass }}$ and $\frac{D_{\text {glass }}}{10}$ diffusion constants give different heat profiles, not consistent with the polarization microscopy images (supplementary information). This confirms that it is the glass substrate, not the metal film, through which the heat transfer mostly occurs and shows the link between heat diffusion and domain wall motion predicted by the model presented.

To find the magnitudes of $F_{u, g}$ from equations 5 and 6 , we need the time dependence of temperature $\mathrm{T}(\mathrm{t})$ and heat fluxes $\vec{J}(t)=-k \vec{\nabla} T$. These are shown for point $\mathrm{A}$ in the lower panels of figure 7. In addition, a quantitative calculation requires the Tdependence of $\sigma_{\omega}$ and $M_{s}$. These quantities cannot be measured in our setup at the high temperatures shown in figure 7. Estimates of $F_{u, g}$, using typical $\sigma_{\omega}(T), M_{s}(T)$ dependencies for magnetic materials in equations 5 and 6 , show values sufficient to overcome the coercive field of several tens of G of sample A. The AOS observed in sample B, which had $H_{c}=2.33 \mathrm{kG}$ in the out-of-plane direction at room temperature, can 
be explained with a decrease of its coercive field at larger temperature. With these forces and a reduced coercive field from heat accumulation, the right-hand side in the velocity equation above becomes positive and the domain size increases. As the sample cools, the forces are reduced, while $H_{c}$ increases back to first stop the DW and, then, to prevent the expanded domains from collapsing. Therefore, the DW motion is not reversible and domains do not contract back on cooling once the laser beam moves away.
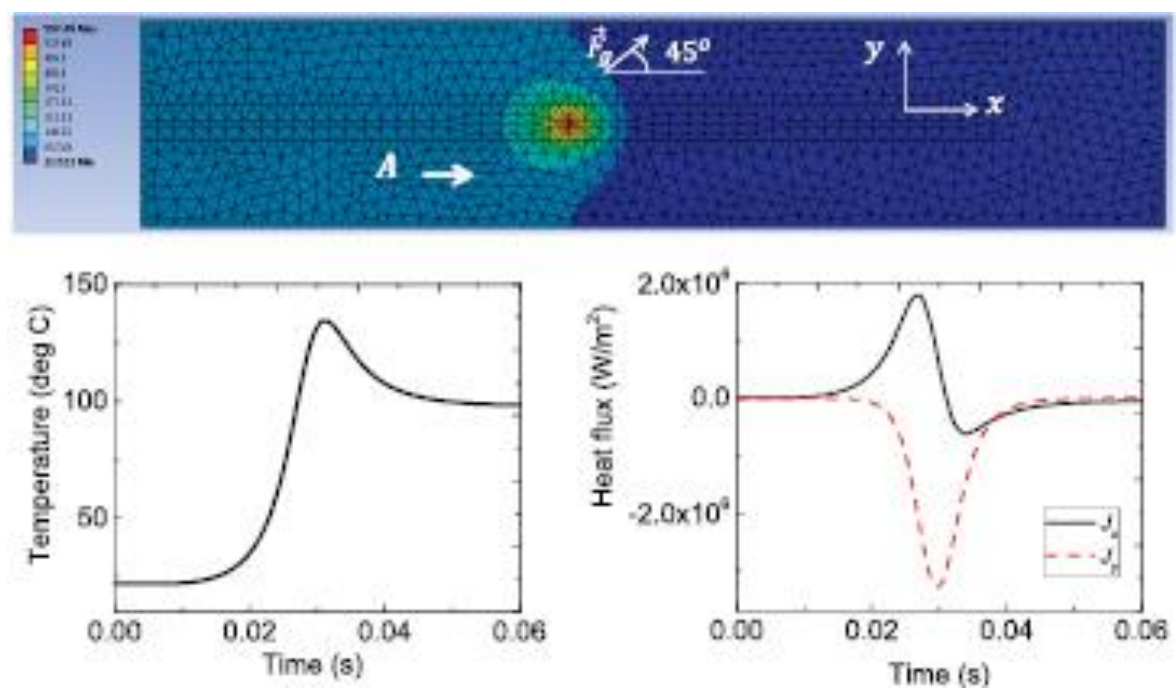

Figure 3.7 | Frame at $\mathrm{t}=37 \mathrm{~ms}$ from results of numerical calculations of the temperature variations of the glass substrate with $D_{\text {glass }}=0.005 \mathrm{~cm}^{2} / \mathrm{s}$, for a $F=5 \times 10^{7} \mathrm{~W} / \mathrm{m}^{2}$ fluence focused in an area $A=30 \mu \mathrm{m} \times 30 \mu \mathrm{m}$. The colour scale is in degrees $C$. The panels show the time-dependence of the temperature and in-plane heat flux at point A on the surface during the beam scan.

This model predicts that AOS is favored in samples with a high mobility $\eta$, a small $M_{s}$ and a small $H_{c}$ at large T. These predictions are consistent with observations. Sample A had a smaller $H_{c}$ at room temperature and, in addition, a smaller thickness $h$. A smaller thickness would give a smaller magnetic anisotropy $\kappa_{1}$ (this dependence was 
observed in $\mathrm{Co} / \mathrm{Pt}[8])$, a larger DW width $\Delta \approx \pi \sqrt{\frac{A}{\kappa_{1}}}$ and larger Bloch DW mobility $\eta \approx$ $\frac{\gamma \Delta}{\pi \alpha}$, where $\gamma, \alpha$ are the parameters in the LLG equation $\frac{d \vec{M}}{d t}=-\gamma \vec{M} \times \overrightarrow{H_{e f f}}+\frac{\alpha}{M_{S}} \vec{M} \times \frac{d \vec{M}}{d t}$ rotating the magnetization at the DW location [45][58]. It would be expected that AOS would be easier to observe in sample A. Indeed, AOS was obtained at lower powers and at the center of the stripe in sample A. Sample B required higher powers and AOS was observed only at the stripe edges, where thermal gradients are larger. Previous measurements are also consistent with this model. For instance, it has been noted that AOS is favored at small $M_{s}$ and $M_{r e m}$ in ferro- and ferrimagnetic materials [21, 59]. This approach should be applicable in other cases [19-21] of large domain wall mobility and more fluid reversal patterns, and is less suitable when multiple domains are observed. Heat accumulation can be estimated for different repetition rates $v_{r e p}$ and pulse energies $E_{\text {pulse }}$ in a simplified model of a sequence of point-like pulses $P(r, t)=A E_{\text {pulse }} \sum_{i} \delta\left(t-t_{i}\right) \delta(r)$. When cooling through the film is neglected, the 3dimensional Green's function $G_{3 D}(r, t)=\frac{1}{(4 \pi D t)^{3 / 2}} e^{-\frac{r^{2}}{4 D t}}$ describes the temperature evolution. The temperature increase at $\mathrm{r}=0$ due to each pulse can be obtained with $G_{3 D}\left(0, t-t_{i}\right)=\frac{1}{\left(4 \pi D\left(t-t_{i}\right)\right)^{3 / 2}}$ from the moment of impact $t_{i}$ to the observation time $t$. The overall increase is given by the sum over contributions from all previous pulses separated by $\Delta t=1 / v_{\text {rep }}$ or $\Delta t \propto \sum_{i} \frac{E_{\text {pulse }}}{\left(t-t_{i}\right)^{3 / 2}}$. Then, immediately before the arrival of a new pulse $\Delta t \propto \xi(3 / 2) E_{\text {pulse }} v_{\text {rep }}{ }^{3 / 2}$, where $\quad \sum_{n, \text { integer }>0} \frac{1}{n^{3 / 2}}=\xi(3 / 2) \approx 2.61$. Assuming the same thermal diffusivity, light absorption, beam power, and focusing comparing the heat accumulation for a beam of $100 \mathrm{~mW}$ power at our $80 \mathrm{MHz}$ rates and 
lower $1 \mathrm{kHz}$ rate gives $\frac{\Delta T_{\text {oscillator }}}{\Delta T_{1 \mathrm{KHz}}} \approx\left(\frac{1.25 \mathrm{~nJ}}{0.1 \mathrm{~mJ}}\right)\left(\frac{80 \mathrm{MHz}}{1 \mathrm{KHz}}\right)^{3 / 2} \approx 280$. A negligible heat accumulation for $0.5 \mathrm{~mJ} / \mathrm{cm}^{2}$ pulses was confirmed in a series of measurements done at 1 $\mathrm{kHz}-0.1 \mathrm{~Hz}$ repetition rates [22]. In contrast, heat accumulation has been observed in pump-probe experiments (not shown) at our repetition rates and pulse energies.

More detailed calculations for a Gaussian beam profile, refining the estimate above obtained for point-like sources, and spanning the current wide range of experimental conditions are in preparation. $\mathrm{Co} / \mathrm{Pd}$ samples with perpendicular magnetic anisotropy have been investigated with $12 \mathrm{~ns}$ pulses [40]. A thermal demagnetization pattern was obtained, but no AOS was reported. This suggests that it is not only the total energy deposited in the material that matters, but also whether it is done sufficiently quickly. The presented images were made long after AOS was complete and many questions remain, in particular on the domain nucleation process. AOS domains gradually emerge from a demagnetized state induced by one pulse in GdFeCo [60] or after several tens of pulses in $\mathrm{Co} / \mathrm{Pt}[22]$. What is this transient state in $\mathrm{Co} / \mathrm{Pd}$ ? We are interested in investigating the relation between the transient temperature and magnetization of this state after a common temperature has been established, and in its dependence on an applied external magnetic field that favors the nucleation and growth of domains [61]. Additional time-resolved experiments will address these questions.

\subsection{Conclusion}

All-optical switching by domain wall motion has been obtained in ferromagnetic $\mathrm{Co} / \mathrm{Pd}$ superlattices with the faster sequence of pulses from a TiS oscillator. Once a domain is nucleated, a relatively slow domain evolution follows. Heat accumulation and 
in-plane diffusion are considered to obtain a force on the domain walls, which pushes the walls from under the laser spot. 


\section{CHAPTER 4: DEMAGNETIZING FIELDS IN ALL-OPTICAL SWITCHING ${ }^{2}$}

\subsection{Introduction}

Ultrafast demagnetization (UDM) [1] is not an ultrafast rotation of magnetization. Instead, the magnetization partially or completely fragments in the strong-pulsed laser field over a demagnetization time $\tau_{M}$. The exchange energy re-assembles the initial magnetization over an equilibration time $\tau_{E}$. Quantum models with spin-flip electron scattering demonstrate links between $\tau_{M, E}$ and spin precession damping rates [62] in transition metal or rare earth materials and alloys [63-66] (figure 1(a), left panel). Dependence of UDM on external magnetic field [67], ambient temperature [3, 8][63, 68], and excitation wavelength $[69,70]$ have been examined. Alternative models $[71,72]$ and transient spin currents [73-77] have been considered. It is established through experiments that it is possible to obtain UDM without direct light interactions. Such experiments include electron diffusion from a heating $\mathrm{Al}$ layer into $\mathrm{Ni}[78,79]$, in $\mathrm{Co} / \mathrm{Pd}$ multilayers [80], and with ballistic electrons through a $\mathrm{Cu}$ layer into $\mathrm{Co} / \mathrm{Pt}$ [81].

On the other hand, all-optical switching (AOS) which is the light induced reversal of magnetization to a single-domain state, has been observed in ferrimagnetic films [82], ferromagnetic films [83-88], and granular media $[84,89,90]$ with perpendicular magnetic anisotropy.

\footnotetext{
${ }^{2}$ F Hoveyda, E Hohenstein, R Judge and S Smadici et al 2017 J. Phys.: Condens. Matter in press https://doi.org/10.1088/1361-648X/aa9e39
} 
It has been shown that a complete UDM occurs at the center of the excitation area before a gradual emergence of AOS (figure 1(a), right panel) [91]. A possible explanation of AOS is a reversal due to the transient magnetic field of the Inverse Faraday Effect, induced by the laser pulse in the material [82, 92-95]. Alternative models include switching due to laser-induced heat observed in ferrimagnets using linearly-polarized light [96-99], the difference in absorption based on magnetic circular dichroism [100102], interaction of spin magnetic moment with electric field of laser, or models specific to granular media [100, 102].

In this work, time-resolved pump-probe measurements were made on $\mathrm{Co} / \mathrm{Pd}$ superlattices that show partial to complete ultrafast demagnetization from heat accumulation. An analytical model and micromagnetic simulations are applied to determine the conditions for demagnetizing fields to nucleate and develop an AOS state from a demagnetized disk.

\subsection{Experiments}

\subsubsection{Setup}

Ferromagnetic $[\mathrm{Co} / \mathrm{Pd}]_{4}$ superlattices were examined, in which cumulative AOS was observed with linearly-polarized light [88]. The samples were $h=4.1 \mathrm{~nm}$ thick with per- pendicular magnetic anisotropy (PMA).

The pump-probe two-frequency setup has a non-collinear geometry, with measurements in transmission at normal incidence (figure 1(b)). The linearly-polarized $800 \mathrm{~nm}$ pump and $400 \mathrm{~nm}$ probe beams were focused to stationary $\mathrm{w}_{0}=125 \mu \mathrm{m}$ and $\mathrm{w}_{1}=80 \mu \mathrm{m}$ spots, respectively, and the delay between the two pulse sequences scanned with a translation stage. 
The sample magnetization was re-initialized between pump pulses with a constant field $|\mathrm{B}|=300 \mathrm{G}$ from two water-cooled coils. The relatively strong damping $\alpha \approx 0.1 \mathrm{in}$ $\mathrm{Co} / \mathrm{Pd}[103,104]$ insures that the magnetization is stable within the $12.5 \mathrm{~ns}$ time interval between pulses. This allows measuring transient processes with the same initial and final states. Measuring the AOS time dependence, with different initial and final states, requires a field pulsed at the TiS laser repetition rate $(80 \mathrm{MHz})$ and cannot be currently done in our setup.

The probe beam was detected with a balanced photodiode connected to a lock-in amplifier with the reference frequency being set at the chopping frequency of the pump beam $(2.069 \mathrm{kHz})$. Intensity and polarization variations arise from temperature and birefringence transients. A configuration with a polarizer and analyzer near crossing was used which minimizes non-magnetic contributions to the signal [4].

\subsubsection{Results}

The dependence of lock-in resultant " $\mathrm{R}$ " on the delay time $\tau$ was measured at two opposite applied fields $B= \pm 300 \mathrm{G}$ for different pump beam powers (figure 2). We used $\mathrm{R}$ to calculate two components; An anti-symmetric part, $A_{R}(\tau)=\frac{1}{2}(R(-B)-R(+B))$, which emboldens the signal features that depend on the B direction and a symmetric part, $S_{R}(\tau)=\frac{1}{2}(R(-B)+R(+B))$ that boosts the features that are independent from B.

The symmetric part $S(\tau)$ shows a prominent peak (corresponding to electron temperature), similar to results for Co films [105], followed by a step (figure 3) (corresponding to the lattice temperature). The dependence of $\mathrm{S}(\tau)$ on delay is similar to the temperature example shown in figure 1(a) for exchange energy between electron and lattice thermal reservoirs in a two-temperature model [106]. 

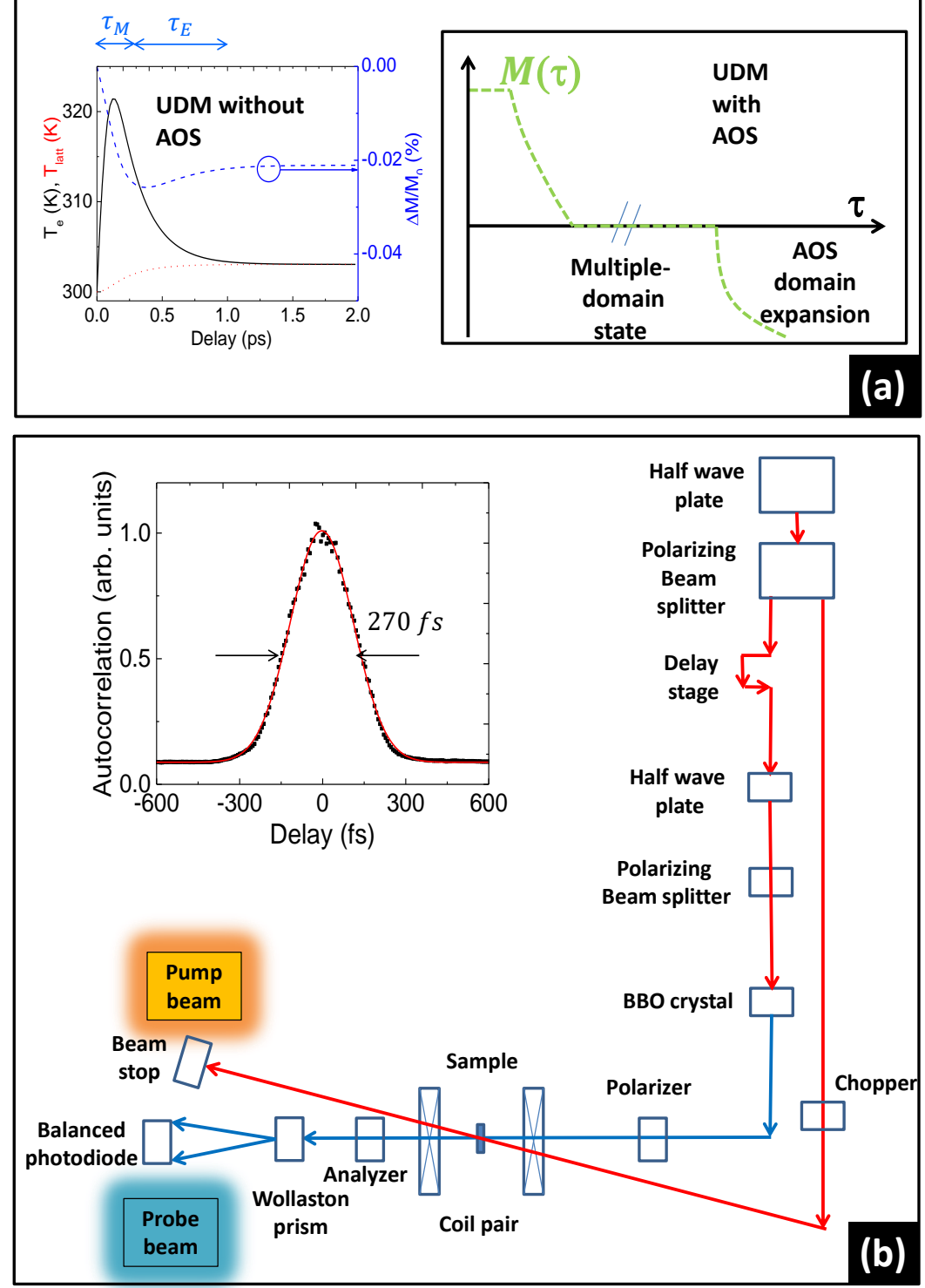

Figure 4.1 | (a) Left panel: $\mathrm{T}_{\mathrm{e}}, \mathrm{T}_{\text {latt }}$ and $\mathrm{M}$ of an ultrafast model for $\mathrm{P}_{\mathrm{abs}}=40 \mathrm{~mW}$ and $\mathrm{h}=4.1 \mathrm{~nm}$ with bulk cobalt parameters $[63,65,76]$, neglecting heat accumulation and electron-induced UDM. Right panel: sketch of the magnetization time-dependence in GdFeCo with AOS domains starting after 10 ps at the center [91]. This takes a msrange time interval in cumulative $\mathrm{AOS}$ of $\mathrm{Co} / \mathrm{Pd}[88]$ and $\mathrm{Co} / \mathrm{Pt}[86]$. (b) Sketch of the experimental setup. Inset: autocorrelation of 190 fs pump pulses at the sample location.

As the experiments are performed in transmission geometry, and also due to the strong metal absorption, a transient plasma from glass substrate [107] contributes to our 
signal even at relatively low fluence. Since glass also contributes and heat accumulation is large, a simpler Gaussian with a step fit has been applied, which describes the experimental results well (figure 3).

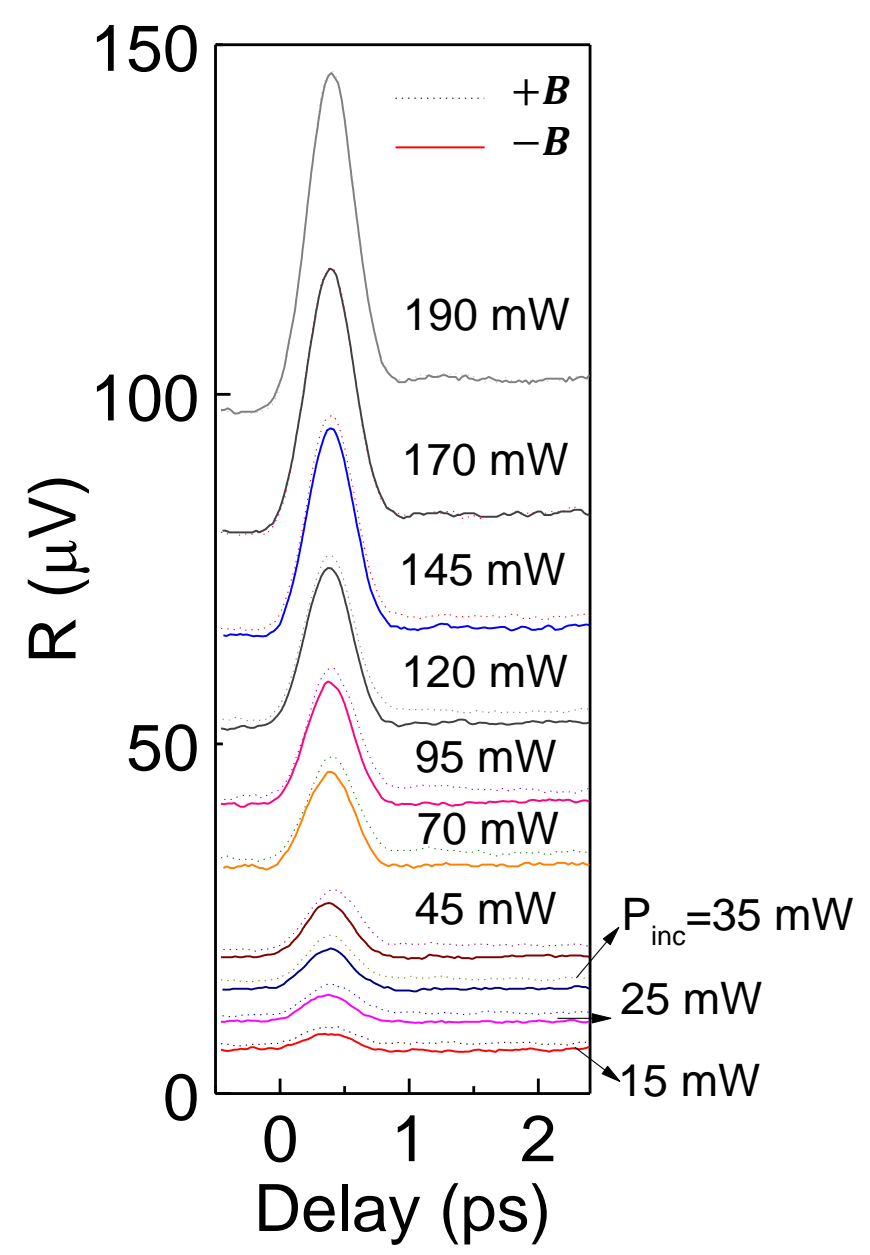

Figure $4.2 \mid \mathrm{R}(\tau)$ for different magnetic fields and incident pump beam power.

The film temperatures can be estimated for $E_{\text {pulse,abs }}=0.5 \mathrm{~nJ}$, corresponding to an absorbed power $P_{a b s}=40 \mathrm{~mW}$. From the energy conservation $T_{e, \max } \approx$ $\sqrt{\frac{2 E_{p u l s e, a b s}}{\gamma h \omega_{0}^{2}}+T_{0}^{2}} \approx 335 \mathrm{~K}$, when neglecting the transfer of energy to the lattice over the duration of the pulse, where $\mathrm{T}_{0}=300 \mathrm{~K}$ is the initial temperature, $\mathrm{C}_{\mathrm{e}}=\gamma \mathrm{T}$ with $\gamma=665$ 
$\mathrm{J} /\left(\mathrm{m}^{3} \mathrm{~K}^{2}\right)$ for bulk Co [65]. Similarly, the lattice temperature step increase after one pulse is $\mathrm{T}_{\text {latt }} \approx$ Epulse $/\left(C_{\text {latt }} h \omega_{0}^{2}\right) \approx 2.5 \mathrm{~K}$. In addition, the heat accumulation temperature $\mathrm{T}_{\text {acc }}$ cannot be neglected for thin samples and high-repetition rate lasers, with multiple pulses incident on the same area within the heat diffusion time $\frac{\omega_{0}^{2}}{4 D}$. The maximum heat accumulation is $\mathrm{T}_{\mathrm{acc}, \max }=310 \mathrm{~K}$ for $\mathrm{P}_{\mathrm{abs}}=40 \mathrm{~mW}, \mathrm{~h}=4.1 \mathrm{~nm}, \mathrm{w}_{0}=125 \mu \mathrm{m}$, an interface conductance $\mathrm{G}>10^{6} \mathrm{~W} / \mathrm{m}^{2} \mathrm{~K}$ and the same thermal parameters as in Ref. [108].

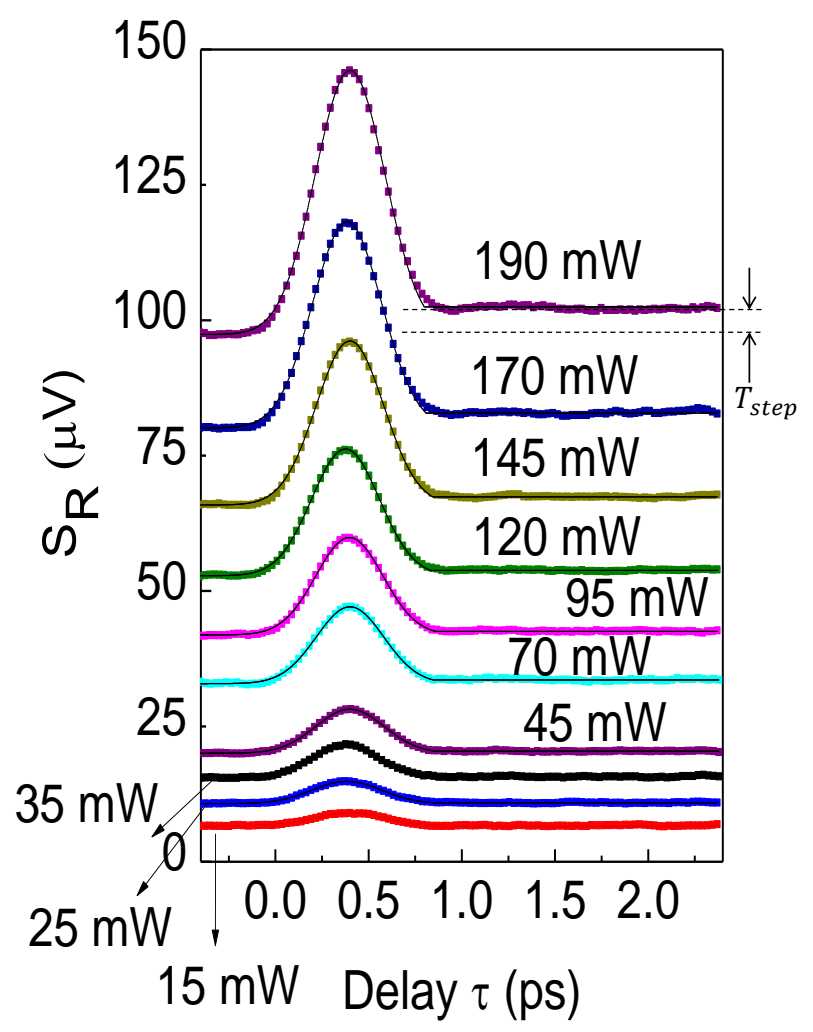

Figure $4.3 \mid \mathrm{S}(\tau)$ at different power and Gaussian with step fit.

The antisymmetric part $\mathrm{A}(\tau)$ (figure 4) increases and then decreases with power. Measurements away from crossing configuration resulted in a featureless $\mathrm{A}(\tau)$. The timedependence corresponds to type I UDM [63], where the demagnetization is followed by recovery of $\mathrm{M}$. The demagnetization time $\tau_{\mathrm{M}}$ was similar to Co and Co/Pt [65], and 
consistent with measurements of UDM in Co/Pd with XMCD [109], XRMS [80], and Xray Fourier transform holography [75]. In contrast to previous measurements, heat accumulation temperature $\mathrm{T}_{\mathrm{acc}}$ is significant and the UDM depth decreases at higher power, as magnetization is gradually removed. Complete demagnetization is obtained at $\mathrm{P}_{\mathrm{inc}}=170 \mathrm{~mW}$ which, from measurements of reflected and transmitted beam powers, corresponds to $\mathrm{P}_{\mathrm{abs}}=40 \mathrm{~mW}$. The heat accumulation temperature for this absorbed power gives a Curie temperature $T_{C}=610 \mathrm{~K}$, consistent with results in similar samples of $\mathrm{T}_{\mathrm{C}}=$ $800 \mathrm{~K}$ for $\mathrm{Co} / \mathrm{Pd}$ and $\mathrm{T}_{\mathrm{C}}=600 \mathrm{~K}$ for $\mathrm{Co} / \mathrm{Pt}[65]$.
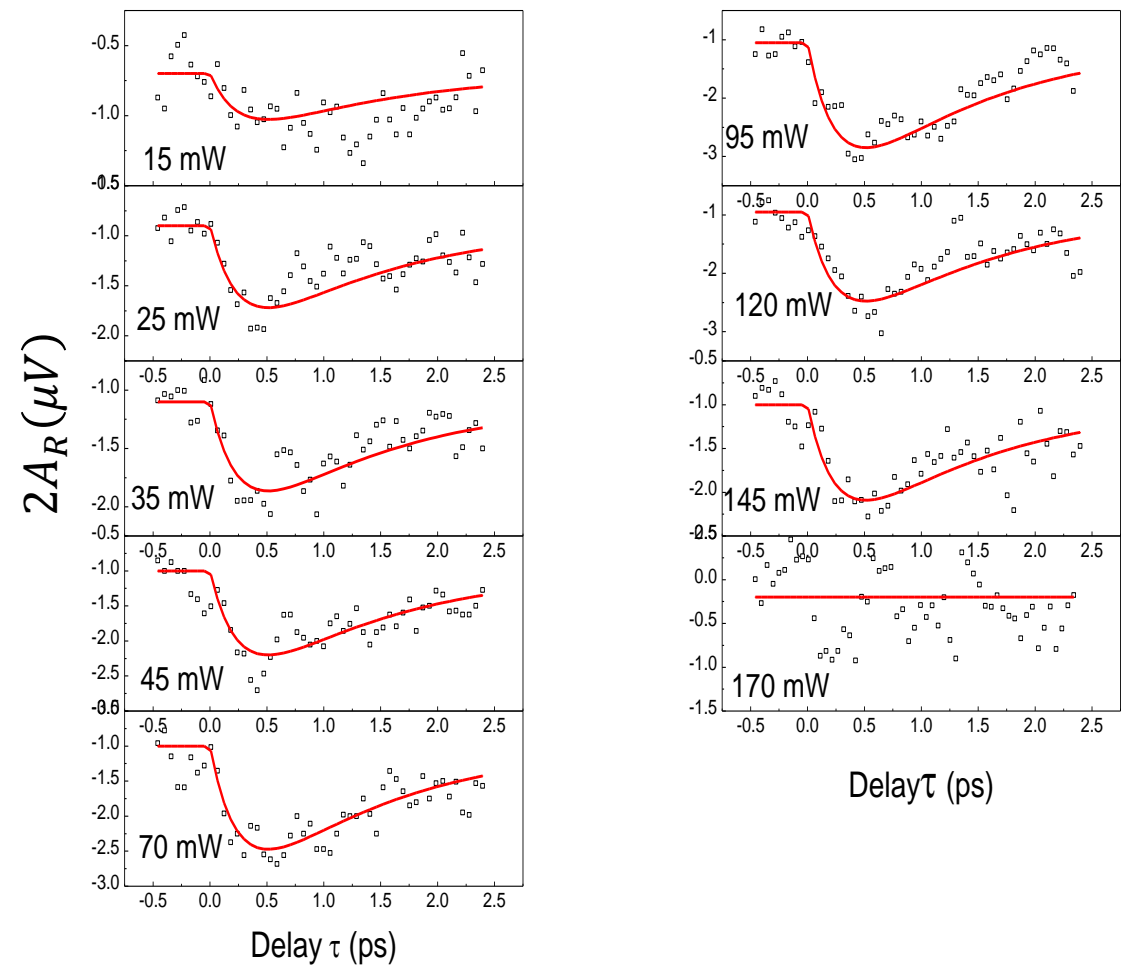

Figure $4.4 \mid \mathrm{A}(\tau)$ at different power and two-exponential fit.

The ultrafast time-evolution of magnetization in magnetic materials depends on the electron $\mathrm{T}_{\mathrm{e}}$ and lattice $\mathrm{T}_{\text {latt }}$ temperatures. Transfer of energy from electrons to lattice includes a spin-flip process in a microscopic scattering model. The rate of spin-flip 
scattering is proportional to the magnetization variation $\mathrm{dM}(\tau) / \mathrm{d} \tau$ [63-65, 92]. Indirect electron-induced UDM from the glass plasma [107] may also contribute, as also seen in $\mathrm{Co} / \mathrm{Pd}$ multilayers capped with $\mathrm{Al}$ [80]. Indirect electron-induced UDM from the glass plasma [107] may also contribute, as also seen in Co/Pd multilayers capped with $\mathrm{Al}$ [80]. For simplicity, a rate equation fit was applied

$$
M(t)=A\left(P, T_{a c c}\right)\left(e^{-\frac{t}{\tau_{E}}}-e^{-\frac{t}{\tau_{M}}}\right) \theta(t)+\text { const }
$$

where $\theta(\mathrm{t})$ is the step-function, and $\tau_{M, E}$ the demagnetization and equilibration times, respectively. The measurements were well fit with $\tau_{M}=0.25 \mathrm{ps}$ and $\tau_{E}=1.3 \mathrm{ps}$. They confirm that demagnetized states are induced in Co/Pd. As in single-pulse AOS of GdFeCo [91] and cumulative AOS of Co/Pt superlattices [86], a demagnetized state is a precursor of cumulative $\mathrm{AOS}$ in $\mathrm{Co} / \mathrm{Pd}$ [88]. The relation between demagnetization and AOS was examined in a model of demagnetizing fields and with micromagnetic simulations.

\subsection{Discussion}

\subsubsection{Micromagnetic simulations}

Demagnetizing (DM) fields express the long-range magnetic moment dipolar interactions, which are relatively weak compared to the short-range exchange interactions. Nevertheless, DM fields and interactions cannot always be neglected, in particular in PMA materials. In practice, they can induce a precession of an intact macrospin [110,111], have been proposed as a driver of AOS [112], and are relevant to AOS in TbFeCo [113, 114], in GdFeCo $[115,116]$, in ferromagnetic Co/Pt $[83,84]$ and $\mathrm{Co} / \mathrm{Pd}[88]$ superlattices. 
A DM field HD will not switch the magnetization spontaneously in a PMA material because the anisotropy field $H_{K}=\frac{2 K}{M_{S}}>4 \pi M_{S}=H_{D}$. However, the initial state after the pump pulse is a demagnetized state, not a spatially-uniform PMA state. Investigating the time evolution of this state requires micromagnetic simulations.

Micromagnetic OOMMF [117] simulations were made, in which the classical macrospin was fragmented into cell spins with the cell size $\mathrm{a}^{3}=(5 \mathrm{~nm})^{3}$. The cell size sets the minimum feature size and can be compared to the Bloch domain wall (DW) width $l_{B}=\sqrt{\frac{A}{K}} \approx 6 \mathrm{~nm}$ for magnetic parameters at the center of the diagram and the width $l_{B}=25 \mathrm{~nm}$ calculated for bulk Co [118]. A demagnetized state is made in a cylinder with a radius comparable to beam size $(\mathrm{R}=3-150 \mu \mathrm{m})$ in AOS experiments. These volumes were impractical to simulate. Simulations of smaller volumes were made and the results compared to an analytical model. An initial random spin state was defined in a cylindrical volume of radius $\mathrm{R}$ and height $\mathrm{h}$ at the center of $\mathrm{a} 1 \times 1 \times \mathrm{h}$ plane (figure $5(\mathrm{a})$ ). Typical values were $\mathrm{R}=50 \mathrm{~nm}, \mathrm{~h}=10 \mathrm{~nm}$, and $\mathrm{l}=1000 \mathrm{~nm}$. Runs with different $\mathrm{a}, \mathrm{R}$ and $\mathrm{h}$ (not shown) gave results consistent with expectations. The DM cylinder is a highenergy state because of spin misalignments.

The time-evolution was examined with an energy minimization solver and the final state identified from its total magnetization normal to the plane $\mathrm{M}_{\mathrm{Z}}$. A timeevolution solver, based on the Landau-Lifshitz- Gilbert equation with a damping constant $\alpha=0.05$, gave similar results.

The time-evolution is determined by macroscopic energy densities $\mathrm{K}, \mathrm{A}, 2 \pi \mathrm{M}_{\mathrm{S}}{ }^{2}$, where $\mathrm{K}$ is an uniaxial anisotropy energy density with an easy axis normal to the surface, 
A is the exchange stiffness $\mathrm{A}=\mathrm{JS}^{2} / \mathrm{d}$, where $\mathrm{J}$ is the nearest neighbor exchange interaction and $d$ the structural unit cell, and $2 \pi \mathrm{M}_{\mathrm{S}}{ }^{2}$ is the DM field energy density. The uniaxial anisotropy $\mathrm{K}$ tends to pull spins out of plane, the exchange interaction $\mathrm{A} / \mathrm{a}^{2}$ tends to keep spins aligned, and the DM field energy $2 \pi \mathrm{M}^{2}$ pulls them in plane. The process was automated and $\mathrm{K}, \mathrm{A}, \mathrm{M}_{\mathrm{S}}$ varied over a wide range. A complex phase diagram may be expected when $K, \frac{A}{a^{2}}$ and $2 \pi \mathrm{M}_{\mathrm{S}}{ }^{2}$ are the same order of magnitude and competing in determining the final magnetic state.

The magnetic structure of the initial state evolves into four different final states of lower energy: (1) a pattern made of small stable clusters, with spins pointing up or down (multiple domain, MD state), (2) an expanding reversed domain (S), (3) a state with spins rotated to an in-plane direction (IP), and (4) a uniform "no-change" state, when the domain closes (NC) (figure 5(a)). The dependence of the final state on $\mathrm{K}$ and $\mathrm{A} / \mathrm{a} 2$ is shown for Ms $=1500 \times 103 \mathrm{~A} / \mathrm{m}$ and $\mathrm{Ms}=500 \times 103 \mathrm{~A} / \mathrm{m}$, with each outcome of a run represented by the symbol at the respective position on the diagram (figure 5(b-c)). A sequence of images (supplementary figure 1) and a movie (supplementary material) show the time-evolution from the initial to the final state. Coalescence of the randomlyoriented spins into a reversed domain $\mathrm{S}$ state and subsequent domain expansion is obtained over a range of $\mathrm{A} / \mathrm{a} 2$ and $\mathrm{K}$. The $\mathrm{S}$ state is not obtained when the DM fields are neglected (not shown). 


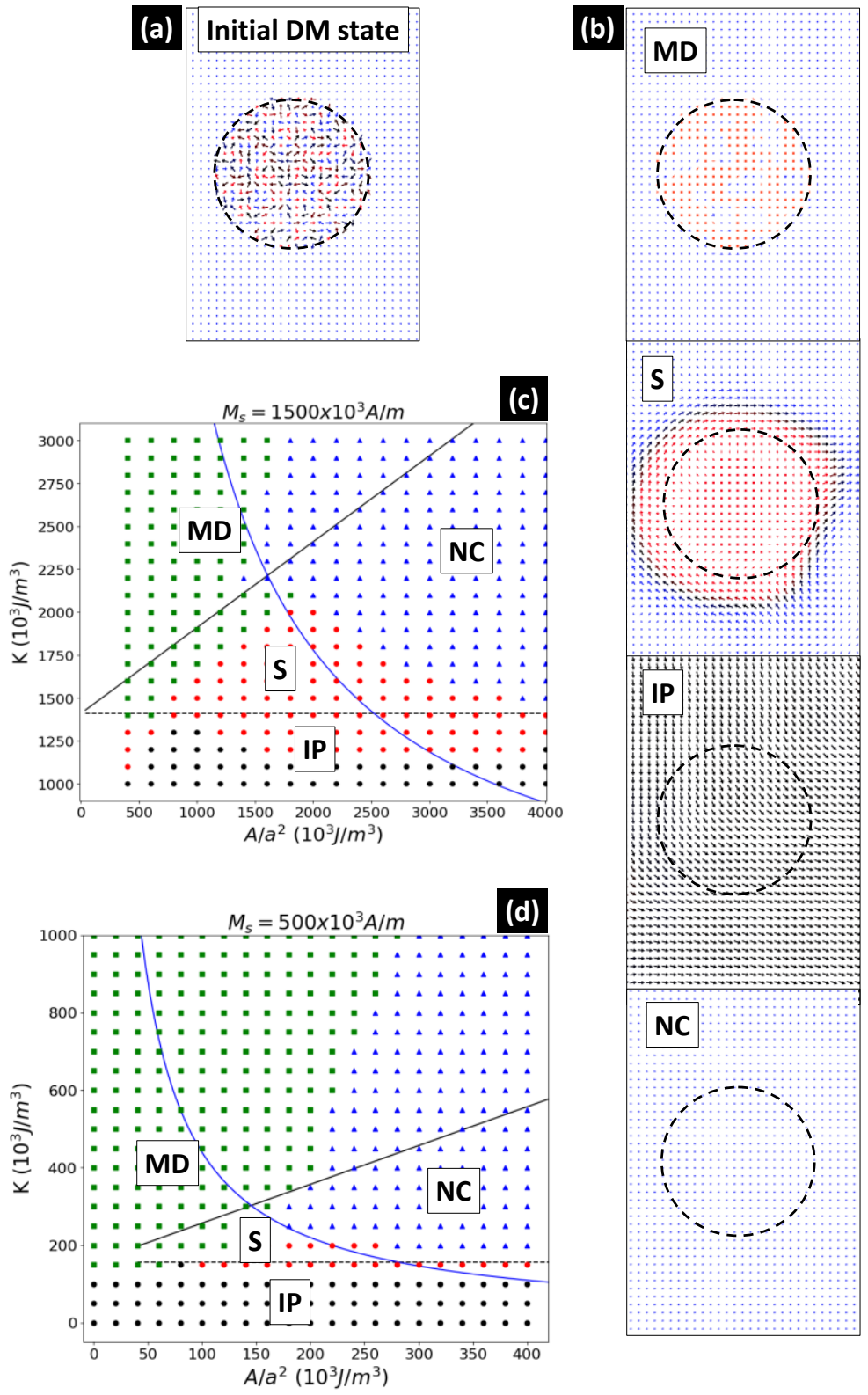

Figure 4.5 | (a) Initial random spin distribution in a $50 \mathrm{~nm}$ radius cylinder. Spins pointing up or down out of plane are labelled blue and red. (b) Examples of a MD final state, S state as domains walls expand outward beyond the radius of the initial cylinder, IP state with spins in-plane, and NC state with all spins pointing up. (c) Simulation results for $\mathrm{M}_{\mathrm{s}}=1500 \times 10^{3} \mathrm{~A} / \mathrm{m}, \mathrm{h}=10 \mathrm{~nm}$, and $\mathrm{R}=50 \mathrm{~nm}$. (d) For $\mathrm{M}_{\mathrm{s}}=500 \times 10^{3} \mathrm{~A} / \mathrm{m}, \mathrm{h}=10 \mathrm{~nm}$ and $\mathrm{R}=50 \mathrm{~nm}$. 
The nucleation and expansion of the $\mathrm{S}$ state in micro- magnetic simulations can be understood in a continuum model. The horizontal line in figure 5(b-c) is the PMA boundary condition $\mathrm{K}=2 \pi \mathrm{M}_{\mathrm{S}}{ }^{2}$, below which the magnetic state has in-plane spins. A multiple domain MD state occurs when $\mathrm{K}$ is large and the two minima for the two spin orientations along the easy axis are separated by a large barrier. Unless A is also large, deepening one minimum over the other, depending on the orientation of neighboring spins, the barrier cannot be overcome. The boundary between the $\mathrm{S}$ and MD states has a positive slope, with progressively larger A required at larger $\mathrm{K}$ to avoid the MD state. The parabola is the condition $P_{\omega}=P_{D}$, where $P_{\omega} \approx \frac{4 \sqrt{A K}}{R}$ is the DW pressure that points inward, due to a reduction of DW energy with a smaller radius. $P_{D}$ is the DM field pressure. Pressures or forces per unit DW area represent the difference in energies on the two sides of a DW [119]. Specifically, if $\Delta \mathrm{E}$ is the difference in the energy density on the two sides of a DW, its displacement over a distance $d$ will change the energy by (Ad) $\Delta E$. This change is equal to the work done by a force $\mathrm{W}=\mathrm{Fd}=(\mathrm{Ap}) \mathrm{d}$ and the pressure on the DW is equal to the difference in the energy density on its two sides $p=\Delta E$. DM fields and pressures have been calculated before for a reversed cylinder in a macroscopic model in analogy to the electric fields of charged layers, with electric charges replaced by magnetic poles and integrating over poles distributed on the two surfaces $H_{D}(z)=$ $\int \frac{M_{S} \cos \alpha d A}{r^{2}}$, where $\alpha$ is the angle from the surface normal (figure 6(a)) [64]. The average over the film thickness $\left\langle H_{D}\right\rangle=\frac{1}{h} \int H_{D}(z) d z$ for a cylinder of reversed magnetization relative to the plane (figure 6(c)) simplifies to $[119,120]$ 
$\left\langle H_{D}\right\rangle=\left\{\begin{array}{c}\frac{M_{S} h}{R}\left(1+2 \ln \frac{8 R}{h}\right), \text { at DW for } R \gg h \\ 4 \pi M_{S}\left(2 \sqrt{1+\frac{R^{2}}{h^{2}}}-\frac{2 R}{h}-1\right), \text { at center }\end{array}\right.$

Then, $p_{D}=2 M_{s}\left\langle H_{D}\right\rangle$ or a force $F_{D}=4 \pi h^{2} M_{s}^{2}(1+2 \ln (8 R / h))$. The DM field points opposite magnetization at DW (figure 6(c)), will tend to reverse it, and push the DW outwards [119]. The DM field remains comparable to external fields known to influence the AOS dynamics in GdFeCo [67] at relatively large $R / h$. For instance, $H_{D} \approx 0.02 \times M_{s} \approx$ $350 \mathrm{G}$ for Co at $R / \mathrm{h}=(10 \mu \mathrm{m}) /(10 \mathrm{~nm})=10^{3}$. The DM field of a demagnetized cylinder (figure 6(d)) can be calculated similarly to give

$\left\langle H_{D}^{\prime}\right\rangle=\left\{\begin{array}{c}2 \pi M_{S}+\frac{h M_{S}}{2 R}\left(1+2 \ln \frac{8 R}{h}\right), \text { at DW for } R \gg h \\ 4 \pi M_{S}\left(2 \sqrt{1+\frac{R^{2}}{h^{2}}}-\frac{R}{h}\right), \text { at center }\end{array}\right.$

where the approximate expression at the DW can be used with an error $<1 \%$ for $R / h>2$. As expected, $\left\langle H_{D}^{\prime}\right\rangle \rightarrow 2 \pi M_{s}$ or half of the uniform layer $4 \pi M_{s}$ at the DW as $R \rightarrow \infty$, and $\left\langle H_{D}^{\prime}\right\rangle \rightarrow 4 \pi M_{s}$ at the center as $R \rightarrow 0$. The case of a demagnetized cylinder is more complex because exchange and anisotropy energies are different on the DW sides. It is also observed that the demagnetized cylinder quickly evolves into a reversed cylinder when the final $S$ state is obtained (supplementary figure 1). The $p_{w}=p_{D}$ condition for $R=50 \mathrm{~nm}, h=10 \mathrm{~nm}$ and $M_{s}=1500 \times 10^{3} \mathrm{~A} / \mathrm{m}$ is plotted in figure 5(b). Above it, the inward DW pressure $p w$ is too large and the domain closes. When $M_{s}$ decreases 3 times, the PMA line decreases 32 times and the parabola decreases 34 times (figure 5(c)). The boundaries of the $N C$ state calculated with the continuum model are consistent with micromagnetic simulations. 
(b)
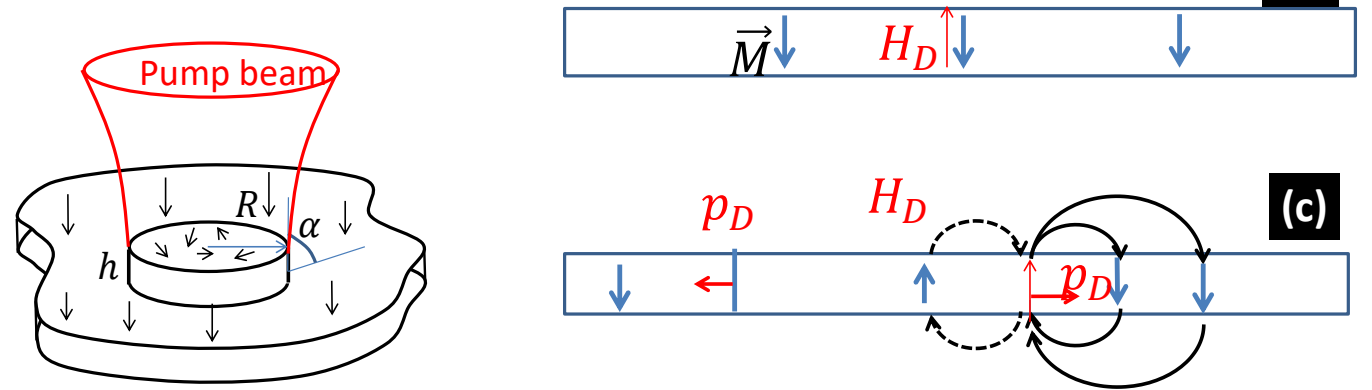

(a)
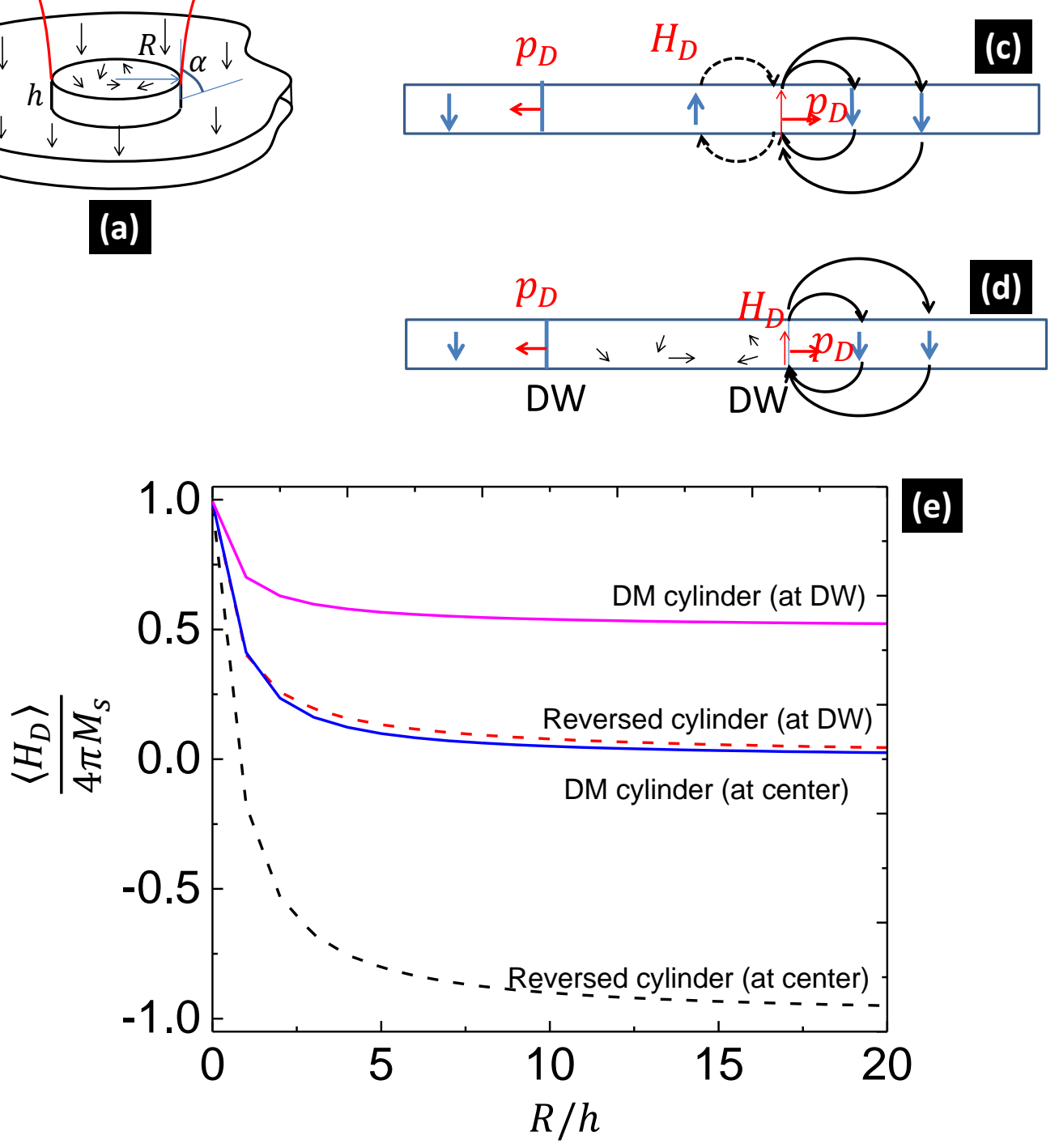

Figure 4.6 | (a) Sketch of a demagnetized cylinder of radius $\mathrm{R}$ and height $\mathrm{h}$ made by the laser beam. (b) Side view of a plane with an uniform PMA magnetic structure with $\left\langle H_{D}\right\rangle=4 \pi M_{s}$ inside and no field lines outside. (c) Field lines for a reversed cylinder, where additional (dotted) fields decrease $\mathrm{H}_{\mathrm{D}}$ compared to the next case. (d) Field lines for a DM cylinder. (e) Dependence on $\mathrm{R} / \mathrm{h}$ of $\mathrm{DM}$ fields at the center and edge of a demagnetized or reversed cylinder. The field at other points is in-between these two limits. Fields of a partially demagnetized cylinder can be obtained by combining these cases. 
The results show that states to the left of $N C$ states and above the PMA line are switched $S$ or multiple domain $M D$ states, depending on $K$. A random spin state in a cylindrical volume is a sufficient precursor of magnetization reversal within a range of $K$ and $A / a^{2}$ energy densities.

\subsubsection{Connection to experiments}

The exchange stiffness $A$ is large in practice, and magnetic materials place to the right of the $S / N C$ boundary in figure $5(\mathrm{~b}-\mathrm{c})$. For instance, bulk cobalt has $\frac{A}{d^{2}} \approx$ $\frac{21 \times 10^{-12} \mathrm{~J} / \mathrm{m}}{\left(2.2 \times 10^{-10} \mathrm{~m}\right)^{2}}=430 \frac{\mathrm{J}}{\mathrm{cm}^{3}}$ at $T \ll T_{c}$, where $d=2.2 \mathrm{~A}$ is the Co lattice constant. The micromagnetic simulations confirm that $50 \mathrm{~nm}$ radius regions with randomly-oriented spins will be closed, or that the PMA ferromagnetic state is stable against these small volume fluctuations, as expected. Volumes with a radius comparable to the beam diameters are inaccessible in simulations. However, the $M D$ and $N C$ states have been observed in AOS experiments with materials above the PMA condition line. For instance, an $M D$ state [84] is obtained when the equilibrium linear domains are smaller than laser spot size [85]. A $N C$ final state has been observed in GdFeCo for beams with $R<5 \mu m$ [121], similar to the case of thicker magnetic bubble materials [122].

This suggests that the $S$ final state of micromagnetic simulations represents the AOS state in these cases. For a narrow range near the $S=N C$ boundary the final state alternated randomly between $S$ and $N C$ in the simulations, similar to the stochastic AOS nucleation in $\mathrm{Co} / \mathrm{Pt}$ [123]. AOS domains induced by DM fields appear in polarizing [113] and XMCD [114] microscopy. A similar sequence of events to that obtained for a $S$ final state (supplementary material) has been observed in time-resolved images of AOS in 
GdFeCo [91]. Cumulative AOS experiments are done with multiple pulses, at nonuniform and time-dependent temperatures, which can increase up to $T_{C}$ (section 2). The micromagnetic energy densities $K, A / a^{2}, 2 \pi M_{s}^{2}$ are quantities averaged over spin fluctuations and depend on temperature. Calculations for bulk Co in equilibrium at one temperature give $A \propto[M(T)]^{1.8}$ and $K \propto[M(T)]^{3}[118]$. The temperature dependence of $K, A, M_{s}^{2}$ can be accounted for with additional forces $F_{u, g}$ in a continuum model, for uniform and spatially-dependent $T_{a c c}$, respectively. If each pulse gives a DM cylinder and reverses the magnetization the net result of multiple pulses incident on the same area would be difficult to predict and depend on factors outside experimental control. Nevertheless, well-defined reversed domains are observed in cumulative AOS. Micromagnetic simulations confirm that DM fields and energies are reduced as the first reversed domain expands beyond the beam footprint and that successive pulses nucleate domains that collapse, leaving a stable reversed domain. AOS was not obtained in $\mathrm{Co} / \mathrm{Pd}$ multilayers when 12 ns pulses were used [124]. This could be due to a large anisotropy energy, placing the material in the $M_{D}$ region, or to the pulse duration. A lower $1.6 \mathrm{ps}$ limit on the pulse duration for AOS in Co/Pt [67][123] and an upper $1.5-15 p s$ limit in GdFeCo [121] have been reported. Future work may apply different pulse durations, with micromagnetic simulations of larger volumes, nonuniform and time-dependent $K, A, M_{s}$, accounting for the variation of accumulation temperature $T_{a c c}$ and a scanning beam.

\section{Conclusion}

Ultrafast demagnetization was observed in $\mathrm{Co} / \mathrm{Pd}$ ferromagnetic superlattices. Heat accumulation at high power removes the magnetization. The role of demagnetizing 
fields in domain nucleation and domain wall motion is quantified with micromagnetic simulations to obtain the conditions for which spins of a small demagnetized cylinder evolve into a reversed domain.
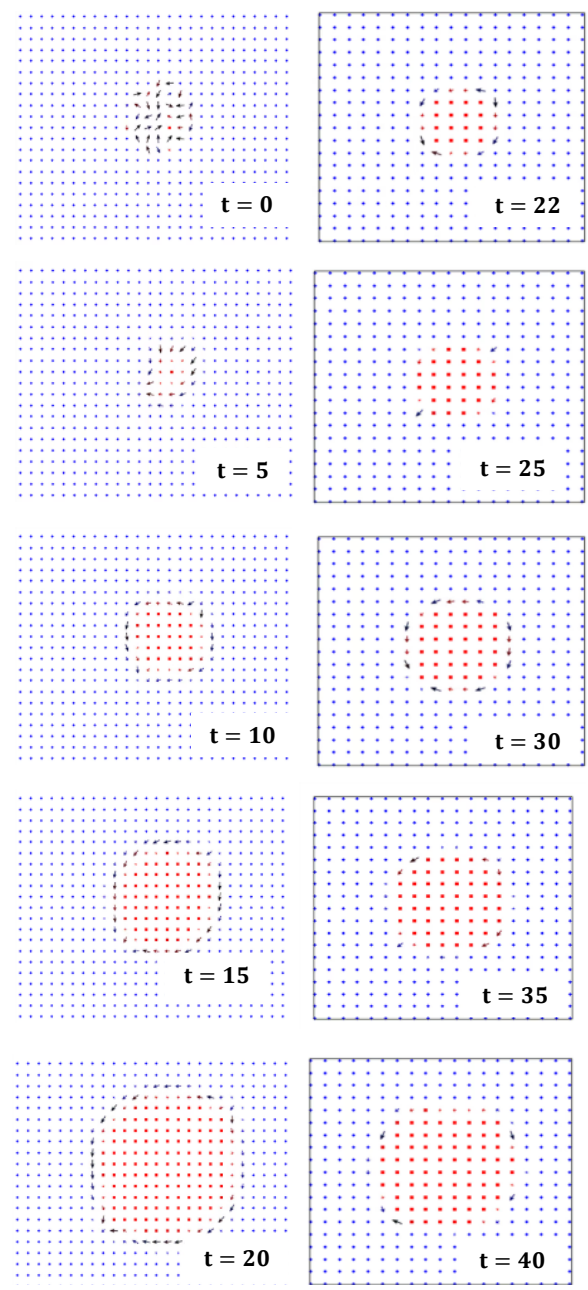

Figure 4.7 | Image sequence of the magnetization time-evolution for one $\mathrm{S}$ point in figure $5(\mathrm{~b})$ at $\mathrm{K}=1800 \times 10^{3} \mathrm{~J} / \mathrm{m}^{3} ; \mathrm{A} / \mathrm{a}^{2}=2400 \times 10^{3} \mathrm{~J} / \mathrm{m}^{3} ; \mathrm{M}_{\mathrm{s}}=1500 \times 10^{3} \mathrm{~A} / \mathrm{m}$. Field of view zooms out at $\mathrm{t}=21$ to a larger area. 


\section{CHAPTER 5: HEAT DIFFUSION IN MAGNETIC SUPERLATTICES ON GLASS SUBSTRATES ${ }^{3}$}

\subsection{INTRODUCTION}

Thermal energy management of opto- and spintronic devices under pulsed laser excitation becomes increasingly important as devices decrease in size. Heat diffusion in multilayer materials can be quantified with modulated thermoreflectance measurements of the temperature spatial profile [125] and frequency dependence [126]. Pump-probe time-domain measurements of the temperature time-evolution, following a TiS laser ultrafast transient disturbance $T_{t r}$, developed into a powerful technique for measuring thermal conductivity and interface conductance [127]. An additional heat accumulation temperature increase $T_{a c c}$, similar to that of modulated thermoreflectance, will also result for these sources, when the thermal energy deposited into a highly absorbing metal layer does not fully dissipate between pulses [128, 129].

A magnetic material saturation magnetization $\mathrm{Ms}$, magnetic anisotropy $\mathrm{K}$, exchange energy $A$, and coercive field $H_{c}$ all depend on $T$. The equilibrium magnetization magnitude and direction is determined by a balance of several energies that depend on these factors and implicitly on temperature. For instance, temperature increases induced by laser beams can modify the equilibrium conditions and start a magnetization precession, switch it between different easy axis minima [130, 131], or

\footnotetext{
3 "Reprinted from [Hoveyda F., Adnani, M., Smadici, S., "Heat diffusion in magnetic superlattices on glass substrates", 2017 J. Appl. Phys. 122 184304], with the permission of AIP Publishing."
} 
modify the magnetization hysteresis loops in applied fields [132]. Magnetic structures induced by laser beams [133-137], all-optical switching (AOS) in thin ferrimagnetic rareearth and ferromagnetic superlattices [138, 139], hybrid structures [140], and granular media [141, 142] have been observed. Local ultrafast [132, 143] as well as thermal models of AOS [144-148] have been proposed. Recently, cumulative AOS in ferrimagnetic rare earth/transition metal, ferromagnetic $\mathrm{Co} / \mathrm{Pt}$ [149], and $\mathrm{Co} / \mathrm{Pd}$ [150] superlattices showed how thermally induced forces can move magnetic domain walls. For a quantitative understanding of these observations, it is necessary to characterize the thermal response of the samples.

In this work, metallic magnetic superlattices were examined with transmission pump-probe measurements of modulated heat accumulation $T_{a c c}$ and polarizing microscopy. Two dimensional heat diffusion and thermal demagnetization patterns illustrate the energy flow in the structures. Green's functions are calculated for different thermal properties and sample geometries in the two experimental configurations of a chopped and a moving beam.

\subsection{EXPERIMENTAL SETUPS}

\subsubsection{Samples}

$[\mathrm{Co} / \mathrm{Ag}]_{3},[\mathrm{Co} / \mathrm{Pd}]_{4}$, and $[\mathrm{Co} / \mathrm{Au}]_{10}$ multilayer samples were deposited with ebeam evaporation on $1 \mathrm{~mm}$ thick soda-lime glass substrates at room temperature. For $\mathrm{Co} / \mathrm{Ag}$, the rates were adjusted to give $1 \mathrm{~nm} \mathrm{Co}$ and $1.4 \mathrm{~nm} \mathrm{Ag}$ individual layer thicknesses, and the sample was capped in-situ with a $\mathrm{SiO} 2$ layer. The $\mathrm{Co} / \mathrm{Pd}$ and $\mathrm{Co} / \mathrm{Au}$ glass substrates were immersed in Nanostrip solution for five minutes, then placed in 
acetone and methanol, and sonicated in each liquid for $10 \mathrm{~min}$ at $60^{\circ} \mathrm{C}$. Hot Nanostrip $\left(90^{\circ} \mathrm{C}\right)$ was used for cleaning the $\mathrm{Co} / \mathrm{Au}$ glass substrates. During the deposition, the substrates were rotated at 5 RPM about an axis making an angle of $45^{\circ}$ with the surface normal. Samples of variable thickness were also made with a custom rotating holder that obscured the source over a position-dependent fraction of the deposition time.

Profilometry measurements on $\mathrm{Co} / \mathrm{Ag}$ showed a maximum total sample thickness of $15.6 \mathrm{~nm}$. The thickness variation along the sample surface was obtained with transmission and reflection measurements with a $633 \mathrm{~nm}$ He-Ne laser. For instance, for $\mathrm{Co} / \mathrm{Ag}$, the thickness decreased linearly along the surface up to $28 \mathrm{~mm}$, beyond which it drops rapidly $[$ Figure $1(\mathrm{~b})$, inset $]$. The $[\mathrm{Co} / \mathrm{Pd}]_{4}$ and $[\mathrm{Co} / \mathrm{Au}]_{10}$ sample thicknesses were 6.2 and $49 \mathrm{~nm}$.

\subsubsection{Pump-probe measurements}

A two-frequency pump-probe setup was applied in a non-collinear transmission geometry. The pump and probe beams were focused on the sample to $\mathrm{w}_{0}=125 \mu \mathrm{m}$ and $\mathrm{w}_{1}=80 \mu \mathrm{m}$ spots, respectively. The linearly polarized pump beam of 120 fs pulses at a repetition rate of $80 \mathrm{MHz}$ and $800 \mathrm{~nm}$ wavelength was chopped, and the polarization variation of a low-fluence, linearly polarized, $400 \mathrm{~nm}$ probe beam was measured with a Wollaston prism, balanced photodiode, and lock-in amplifier. The probe beam was aligned along the surface normal. The incidence angle of the pump beam was $10^{\circ}$ relative to the surface normal. A stage varied the delay between the two pulse sequences [Figure $1(\mathrm{a})]$. 

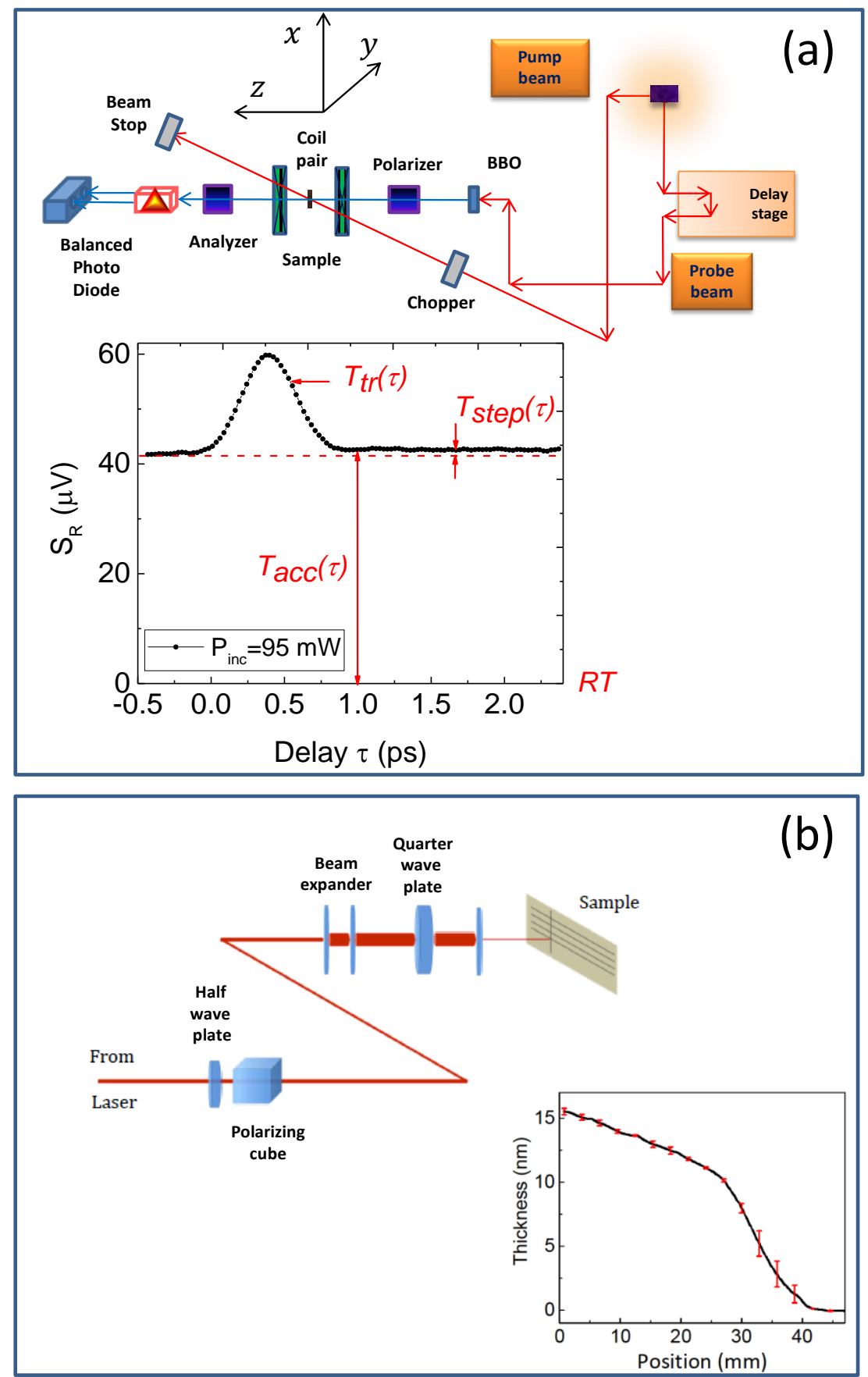

Figure 5.1 (a) Sketch of the pump-probe setup. Inset: the response of the sample symmetric in applied field $S(\tau)=\frac{1}{2}(S(+B)+S(-B))$ measured at $f_{c h}=2.069 \mathrm{KHz}$. (b) The scanning setup with the $\mathrm{Co} / \mathrm{Ag}$ sample thickness profile in the inset. 
Heat accumulation occurs when the interval between consecutive pulses $\frac{1}{f_{\text {rep }}}=$ $12.5 \mathrm{~ns}$ is smaller than the time it takes heat to diffuse out of the beam footprint $\frac{w_{0}^{2}}{4 D}=\frac{(125 \mu \mathrm{m})^{2}}{0.4 \mathrm{~cm}^{2} / \mathrm{s}}=0.4 \mathrm{~ms}$ with typical metal diffusivity. The temperature $T(t)=T_{t r}+$ $T_{a c c}$ is made of two components: a transient $T_{t r}$ peak, related to ultrafast non-thermal processes, and a heat accumulation $T_{a c c}$ from the cumulative effect of multiple pulses, as illustrated by a time-resolved measurement [Figure 1(a), inset]. $T_{\text {step }}$ is the small temperature increase due to one pulse that dissipates before the next pulse arrives and, as expected from the estimate above, is only a small fraction of $T_{a c c}$.

The pump $F_{0}(\omega)$ and probe $F_{1}(\omega)$ beam fluences have the spectrum of the femtosecond comb. In thermoreflectance experiments, a sinusoidal intensity modulation of large frequency ( $>0.1 \mathrm{MHz})$ is usually applied [27][151]. In our experiments, we vary the pump beam fluence with a square-wave chopper modulation. This introduces additional sidebands in the spectrum at $n f_{c h}$, where $\mathrm{n}$ is an integer. However, the lock-in detects at the chopper modulation frequency $f_{c h}$. This is equivalent to replacing the pump pulse sequence with $F_{0}(\omega) \rightarrow F_{0}\left(\omega_{c h}\right)$, keeping only the temporal profile at the frequency $f_{c h}=\frac{\omega_{c h}}{2 \pi}$, and the probe pulse sequence with the average fluence $F_{1}(t) \rightarrow$ const [28][152]. The pump fluence modulation gives a relatively slowly varying $T_{a c c}$ oscillation which, as expected, does not depend on delay within a few tens of ps, either before or after the overlap. 

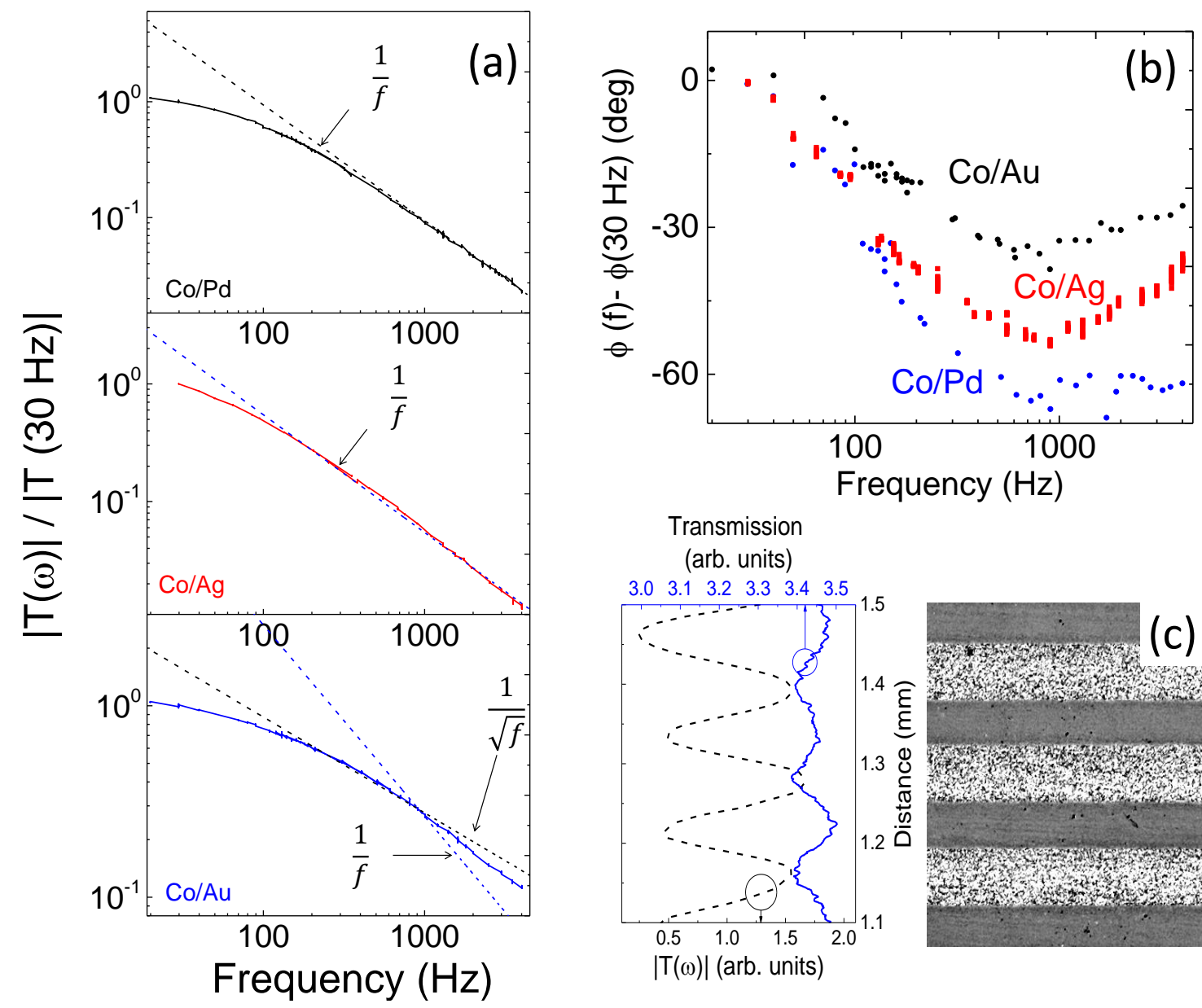

Transmission

(arb. units)
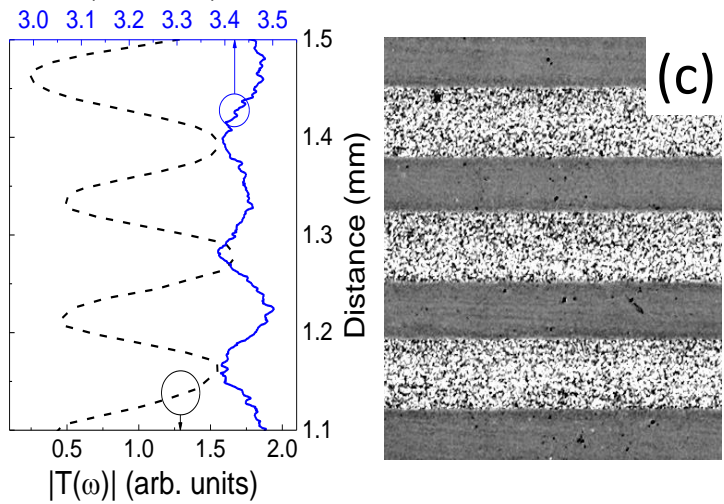

Figure $5.2 \mid$ (a) Temperature modulation amplitude $|T(\omega)|$ dependence on $f_{c h}=\frac{\omega_{c h}}{2 \pi}$, normalized to the value at $30 \mathrm{~Hz}$, for $\mathrm{Co} / \mathrm{Pd}(40 \mathrm{~mW}$ incident power), $\mathrm{Co} / \mathrm{Ag}(46 \mathrm{~mW})$, and $\mathrm{Co} / \mathrm{Au}(40 \mathrm{~mW})$. Similar results were obtained for other powers between $20 \mathrm{~mW}$ and $60 \mathrm{~mW}$. (b)Phase dependence on chopper frequency $f_{c h}$. (c)Light transmission is higher at stripe locations and correlates with variations in $|T(\omega)|$ across $\mathrm{Co} / \mathrm{Ag}$ stripes.

An analysis similar to that done in $\omega, \mathrm{k}$ variables for time-resolved pump-probe measurements can be applied [151]. Specifically, the absorbed fraction of the incident pump pulse fluence $F_{0}(\omega, \mathrm{k})$ is converted to an initial temperature $T_{\text {initial }}(\omega, k)=$ $\frac{F_{0, a b s}(\omega, k)}{C d}$ with the area specific heat $\mathrm{Cd}$, where $\mathrm{C}$ is the volume specific heat. This temperature evolves to a final temperature $T(\omega, \mathrm{k})$ with the Green's function $\mathbb{G}(\omega, \mathrm{k})$ 
representing the heat diffusion. The final temperature $T(\omega, \mathrm{k})$ is sampled by the probe pulse $F_{1}(\omega, \mathrm{k})$ into a spatially averaged temperature $\mathrm{T}(\omega)$.

We apply a Faraday Effect transmission geometry for our semi-transparent samples, complementary to the Kerr Effect reflection geometry on magnetic layers [153] and detect intensity and polarization variations. Then, the lock-in amplifier signal is

$$
\begin{aligned}
L\left(\omega_{c h}\right) & =A \mathbb{T}^{\prime}\left(T\left(\omega_{c h}\right)\right)=B T\left(\omega_{c h}\right) \\
& =B \int d k k F_{1}(k) T\left(\omega_{c h}, k\right) \\
& =B \int d k k F_{1}(k) \frac{F_{0, a b s}(k)}{C d} \mathbb{G}\left(\omega_{c h}, k\right)
\end{aligned}
$$

where $F_{0,1}(k)$ are the Hankel transform of the two beams spatial profiles, the spatial averaging is done by combining the pump and probe beam profiles into a $\mathrm{dk}$ integral[151], and $\mathbb{T}^{\prime}$ is one of the several material properties that depend on temperature. The constant A accounts for different units of $\mathrm{L}$ (in volts) and $\mathbb{T}^{\prime}$. The non-linear terms in $\mathbb{T}^{\prime}(\mathrm{T})$ have been removed since they give a signal at multiples of $f_{c h}$ and $B \equiv A \frac{d \mathbb{T}^{\prime}}{d T}$.

Part of the signal is proportional to the polarization rotation $\theta_{M}=-\frac{\pi d}{\lambda} n Q_{z}$ for a beam propagating along the z-axis due to a magnetization $M_{z} \propto Q_{z}$, where $Q_{z}$ is the offdiagonal magneto-optical coefficient in the susceptibility matrix [154]. This part is antisymmetric in B, can be removed in a combination $S=\frac{1}{2}(S(+B)+S(-B))$, is relatively small [155] and is neglected here. The part of the signal symmetric in B is a thermal modulation of the transmittance $\mathbb{T}$, due to the temperature coefficient of refractive index $\frac{d n}{d T}$. The factor $\frac{d \mathbb{T}}{d T}$ may be called "thermo-transmittance," by analogy with the complementary thermoreflectance $\frac{d \mathrm{R}}{d T}$, which has been examined in detail for a series of materials[32][156] at different wavelengths[33][157]. For metals, $\frac{d n}{d T}=1-5 \times$ 
$10^{-5} K^{-1},[156,157]$ significantly larger than $\frac{d n}{d T}=2 \times 10^{-6} K^{-1}$ for our $(17.5 \% \mathrm{Na} 2 \mathrm{O}$, 7.5\% $\mathrm{CaO}$, and $75 \% \mathrm{SiO} 2$ )soda-lime glass substrate [158]. We then obtain a signal which is proportional to the temperature of the metallic film, where the z-dependence of temperature can be neglected inside our thin thermally conducting samples.

\subsubsection{Scanning measurements}

The beam was expanded and focused on the front of the sample surface with a 30 $\mathrm{mm}$ lens to a typical $\mathrm{w}_{0}=50 \mu \mathrm{m}$ spot [Figure $\left.1(\mathrm{~b})\right]$. The sample was scanned under the beam at a speed $v_{s}=10 \mathrm{~mm} / \mathrm{s}$ at constant fluence. Fluence was adjusted between scans with a half-wave plate and polarizing cube combination. Polarization was adjusted from linear to left and right-circular polarized with a quarter wave plate. No variation was observed with changes in beam polarization.

Polarizing microscopy images were made in transmission Faraday geometry at normal incidence. In contrast to rotation $\theta_{M}$ from magnetization-induced birefringence, the rotation due to structural birefringence depends on the orientation of sample birefringence axes and can be varied with sample rotation.

New areas are continuously exposed in writing experiments with a moving beam.

The diffusion time $\frac{w_{0}^{2}}{4 D}=0.4 \mathrm{~ms}$ out of the beam footprint is comparable to the moving beam dwell time $\tau_{l}=\frac{w_{0}}{v_{s}}=5 \mathrm{~ms}$. The larger radius observed at the end of the stripe [Figure 3(c)] confirms that the steady-state is not obtained during scanning. 

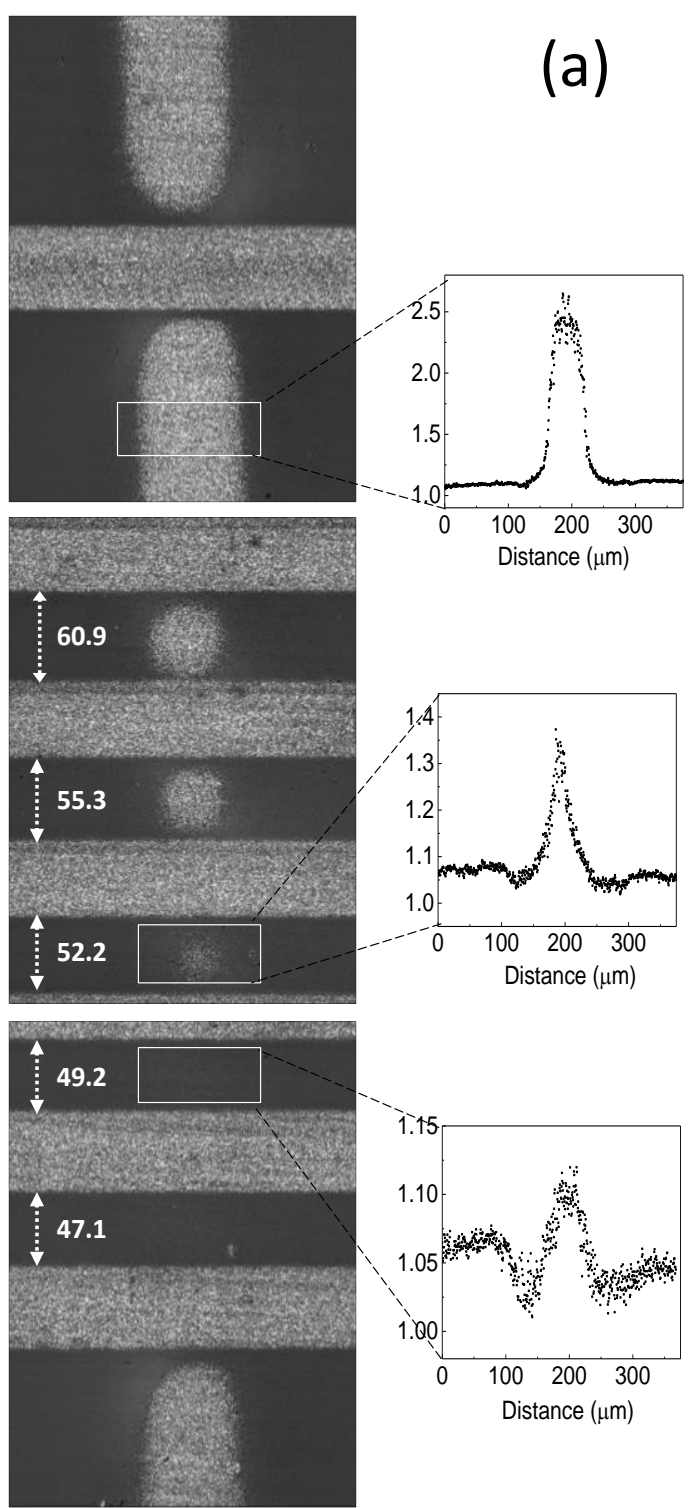

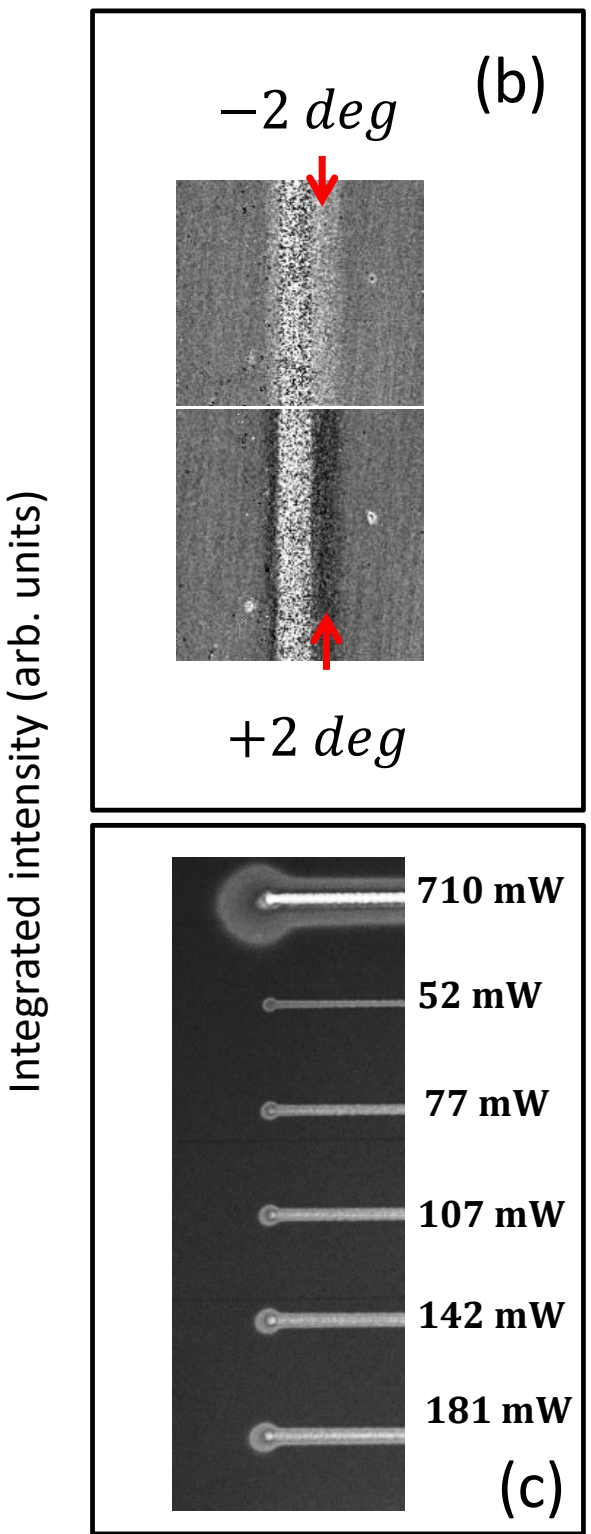

Figure 5.3 | (a) Vertical stripe made in $\mathrm{Co} / \mathrm{Ag}$ across a stack of horizontal stripes, with distances shown in $\mu \mathrm{m}$. Integrated intensity profiles show the stripe almost completely disappears when inside the stack, while the edges are relatively unaffected. (b) Structural birefringence at stripe edges. (c)End of stripe for $\mathrm{Co} / \mathrm{Au}$ at different incident power shows the steady-state condition is not obtained during scanning. 


\subsection{RESULTS}

Measurements of temperature oscillation amplitude $|T(\omega)|$ and phase $\phi(\omega)$ dependence on the chopper frequency $f=\frac{\omega}{2 \pi}$ were made at a $\tau=-2 p s$ delay. Amplitude and phase are plotted relative to measurements at $30 \mathrm{~Hz}$. The amplitude decreases inversely proportional to $\mathrm{f}$ above $200 \mathrm{~Hz}$ for $\mathrm{Co} / \mathrm{Pd}$ and $\mathrm{Co} / \mathrm{Ag}$ and in-between $\frac{1}{f}$ and $1 / \sqrt{f}$ for $\mathrm{Co} / \mathrm{Au}$ [Figure 2(a)]. A levelling of the amplitude is observed for frequencies below $200 \mathrm{~Hz}$. The phase $\phi(\omega)$ also varies with $\mathrm{f}$, first decreasing and then slightly increasing, with a minimum at $\mathrm{f}=1000 \mathrm{~Hz}$.

Beam scans across the surface give "stripes," with small white and black dots at stripe center corresponding to small magnetic domains oriented up and down and domain walls pinned by imperfections [Figure 2(c)]. Pump-probe measurements showed a reduced modulation amplitude $|T(\omega)|$ at stripe locations, and light transmission measurements, made with a small intensity $800 \mathrm{~nm}$ TiS beam, confirmed that these locations have a smaller absorption $F_{0, a b s}$ compared to pristine areas [Figure 2(c)]. A second stripe, made at constant fluence and sample thickness, gradually disappears when intersecting a stack of stripes [Figure 3(a)] and re-appears intact once the stack is crossed.

Stripe edges are birefringent, as shown by intensity variations as the analyzer is rotated across the extinction condition [Figure 3(b) and supplementary material Figure 1]. The birefringence is structural, since it varies from bright to dark over a $90^{\circ} 1$ sample rotation angle. The orientation of the sample birefringence axes depends on the orientation of light polarization when writing the stripes. As expected, birefringence is absent at the intersection of two stripes, made with light beams with orthogonal 
polarizations (not shown). As light $30 \mathrm{~nm}$ bulging was also observed with AFM across stripes made at high power. Similar results were obtained for the other samples.

Isotropic and birefringent modifications can be made in clear glass with amplified TiS lasers following nonlinear multi-photon absorption [159]. Cumulative heating has been considered for isotropic structural changes made with un-amplified lasers [160, 161]. The higher fluence birefringence can arise from stress or $1 \mathrm{~m}$-size elongated voids made in explosive processes of multiphoton and avalanche ionization $[162,163]$. No signal is detected from clear glass for our relatively low fluence and negligible non-linear absorption. Therefore, because linear light absorption in the metal film is required, laserinduced changes in the glass substrate are made in the immediate vicinity of the metal film.

\subsection{DISCUSSION}

\section{A. Heat diffusion in multilayers}

Ultrafast processes occur within the first few ps, until equilibration to a common temperature $T=T_{e}=T_{\text {latt }}$. The subsequent time-evolution of $\mathrm{T}$ in the structure is determined by the heat diffusion equation. It is advantageous to solve this equation following the method applied in time-resolved thermoreflectance in cylindrical symmetry, with new $(\omega, \mathrm{k}, \mathrm{z})$ variables replacing $(\mathrm{t}, \mathrm{r}, \mathrm{z})$. The small ellipticity of the pump beam footprint is neglected. For instance, for the temperature, $\mathrm{k}$ and $\mathrm{r}$ are related as

$$
T(t, r, z)=\int_{0}^{\infty} d k k J_{0}(k r) \int_{-\infty}^{+\infty} e^{i \omega t} T(\omega, k, z)
$$

where $J_{0}(x)$ is the Bessel function [151]. The reverse $\mathrm{r} \rightarrow \mathrm{k}$ transform is $T(k)=$ $\int_{0}^{\infty} d r r J_{0}(k r) T(r)$. Time $\mathrm{t}$ and $\omega=2 \pi f$ are related by the usual Fourier transform. The 
heat diffusion equation with no sources becomes in these variables $\frac{\partial^{2} T(\omega, k, z)}{\partial z^{2}}=$ $q^{2} T(\omega, k, z)$, where $q^{2}=\frac{\Lambda_{\|} k^{2}+i \omega C}{\Lambda_{\perp}}=k^{2}+\frac{i \omega}{D}, \Lambda_{\|}=\Lambda_{\perp}$ are the thermal conductivities parallel and perpendicular to the surface, and $\mathrm{C}$ is the volume specific heat. Its solutions are hyperbolic trigonometric functions that can be arranged in a matrix, describing how the temperature and flux vary with depth $\mathrm{z}$.

Similar transformations are applied to all functions of $\mathrm{t}$ and $\mathrm{r}$, in particular, to the beam profiles. The spatial dependence of a Gaussian beam fluence $F(t, r)=$ $F_{\text {peak }} e^{-2 r^{2} / w_{0}^{2}}=\frac{2 P_{a b s}}{\pi w_{0}^{2}} e^{-2 r^{2} / w_{0}^{2}}$, where $F_{\text {peak }}$ has been replaced with the absorbed Power $P_{a b s}=\int_{0}^{\infty} d r 2 \pi r F_{\text {peak }} e^{-2 r^{2} / w_{0}^{2}}=\frac{\pi w_{0}^{2} F_{p e a k}}{2}$, has a Hankel transform $F(\omega, k)=$ $\frac{P_{a b s}}{2 \pi} e^{-\frac{k^{2} w_{0}^{2}}{8}}$. The in-plane averaging of the pump-induced temperature by the probe is a pump-probe profile convolution in real space or a multiplication in k-space, giving an effective diameter $w_{e f f}=\sqrt{w_{0}^{2}+w_{1}^{2}}=150 \mu m$. Then, the characteristic magnitude of $\mathrm{k}$ in our case is $k_{\text {eff }}=\frac{4}{150} \mu m^{-1} \approx 0.025 \mu m^{-1}$. 

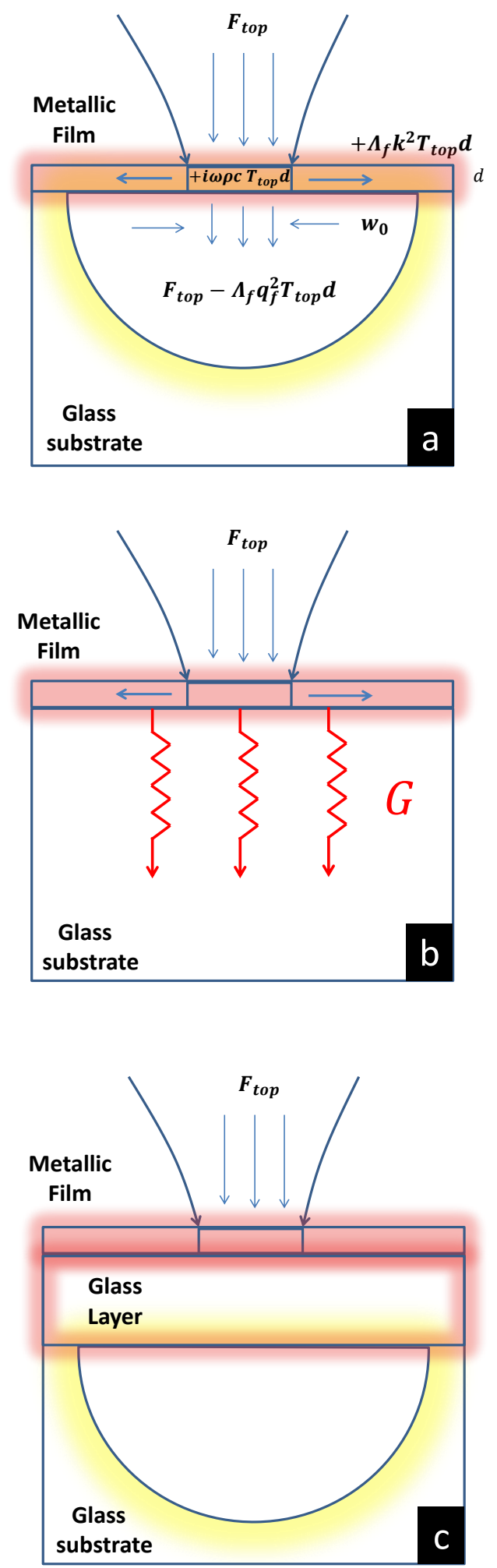

Figure 5.4 | (a) Conducting interface (case A) with cooling rates limited by the substrate. (b)A resistive interface (case B) limits the cooling rate. (c) Two layer model, with a metallic and a glass film. 
The fluxes $F_{t, b}$ and temperatures $T_{t, b}$ on the top (front) and back sides of a multilayer in the limit of a thermally thin film $\left(q_{f} d_{f}=q_{f} d \ll 1\right)$ and thermally thick substrate $\left(q_{s} d_{s} \gg 1\right)$ are related by

$$
\left(\begin{array}{c}
T_{b} \\
F_{b}
\end{array}\right)=\frac{e^{q_{s} d_{s}}}{2}\left(\begin{array}{cc}
1 & -\frac{1}{\Lambda_{s} q_{s}} \\
-\Lambda_{s} q_{s} & 1
\end{array}\right)\left(\begin{array}{cc}
1 & -\frac{1}{G} \\
0 & 1
\end{array}\right) \times\left(\begin{array}{cc}
1 & -\frac{d}{\Lambda_{f}} \\
-\Lambda_{f} q_{f}^{2} d & 1
\end{array}\right)\left(\begin{array}{l}
T_{t o p} \\
F_{\text {top }}
\end{array}\right)
$$

where the substrate, interface conductance G, and the superlattice (replaced with a film) are each represented by a matrix. The film is optically thin and its approximately uniform absorption $(\propto d \ll 1)$ can be replaced by surface absorption $(\propto d \gg 1$, where $\propto$ is the absorption coefficient) as we consider only processes on a longer than $\frac{\mathrm{d}^{2}}{D} \approx \frac{(10 \mathrm{~nm})^{2}}{0.1 \frac{\mathrm{cm}^{2}}{\mathrm{~s}}}=$ 10 ps timescale it takes heat to diffuse through the film. For simplicity, it is assumed first that the thermal properties of the glass near the metal film remain similar to those of the substrate. The condition $F_{b}=0$ gives (a term $\frac{\Lambda_{s} q_{s} d}{\Lambda_{f}} \ll 1$ in the denominator can be neglected)

$$
\begin{aligned}
T_{\text {top }}(\omega, k) & =F_{\text {top }}(\omega, k) \frac{1+\frac{\Lambda_{s} q_{s}}{G}}{\Lambda_{s} q_{s}+\left(1+\frac{\Lambda_{s} q_{s}}{G}\right) \Lambda_{f} q_{f}^{2} d} \\
& =\left\{\begin{array}{cl}
\frac{F_{\text {top }}}{\Lambda_{f} q_{f}^{2} d+\Lambda_{s} q_{s}}, & \text { if } \Lambda_{s} q_{s} \ll G(A) \\
\frac{F_{\text {top }}}{\Lambda_{f} q_{f}^{2} d+G}, & \text { if } \Lambda_{s} q_{s} \gg G(B)
\end{array}\right.
\end{aligned}
$$

where two specific cases have been emphasized (Figure 4). The incident heat flows along different paths, depending on the relation between the interface conductance $G$ and the substrate $\Lambda_{s} q_{s}$ 
In case A with no significant interface backscattering $(G \rightarrow \infty$ and identity interface matrix), cooling rates are limited by the substrate. The term $\Lambda_{f} q_{f}^{2} T_{\text {top }} d=$ $\Lambda_{f}\left(k^{2}+\frac{i \omega C}{\Lambda_{f}}\right) T_{\text {top }} d$ represents the fraction of the incident flux that is carried away sideways in the film $\Lambda_{f} k^{2} T_{\text {top }} d$ or heats the film $i \omega C T_{\text {top }} d$ [Figure 4(a)]. Neglecting this term gives $T_{\text {top }}=\frac{F_{t o p}}{\Lambda_{s} q_{s}}$, the solution for a semi-infinite substrate with surface absorption, with a frequency dependence $T_{\text {top }} \propto \frac{1}{\sqrt{f}}$.

In case $\mathrm{B}$, the interface conductance $\mathrm{G}$ is low, limiting the cooling rate into the substrate. Then, $T_{t o p}=\frac{F_{t o p}}{\Lambda_{f} k^{2} d+\frac{i \omega \Lambda_{f} d}{D}+G}$. As above, the first two terms in the denominator represents sideways film flux and stored heat variations.

Unlike, $\Lambda_{f} k^{2} d$ the new term $\mathrm{G}$ remains finite as $k \rightarrow 0$ toward the peak of the pump profile, always giving a heat flow and removing the temperature divergence of the two dimensional film in the steady-state (Sec. IV C). This term can be absorbed into the $\frac{i \omega \Lambda_{f} d}{D}$ factor, giving $\mathrm{x}$ an imaginary part $i \frac{D G}{\Lambda_{f} d}$. This becomes an exponential $e^{-t / \tau_{G}}$ with the time constant $\tau_{G}=\frac{\Lambda_{f} d}{G D}=\frac{C d}{G}$ following a Fourier transform, describing in the time domain the additional heat transfer channel opened through the interface.

The results can be slightly modified by replacing $F_{\text {top }}$ with the initial temperature $T_{\text {initial }}=F_{\text {top }} / C d$. Then, the expressions in Eq. (4) relate $T_{\text {final }}$ and $T_{\text {initial }}$ and are the Green's functions in $\omega, \mathrm{k}$ variables for different experimental conditions. For instance, $\mathbb{G}_{3 D}(\omega, k)=\frac{1}{\Lambda_{s} q_{s}}$ (obtained in case A in the limit $\left.\mathrm{d} \rightarrow 0\right)$ is the three-dimensional Green's function for the substrate [151]. Similarly, $\mathbb{G}_{2 D}(\omega, k)=\frac{1}{D q_{f}^{2}}=\frac{1}{D k^{2}+i \omega}$ (case B in the limit 
$\mathrm{G} \rightarrow \infty$ ) is the two-dimensional Green's function for the film. This is confirmed by a Hankel in $\mathrm{k}$ and Fourier in $\omega$ transform of $\mathbb{G}_{2 D}(\omega, k)$ that gives

$$
\mathbb{G}_{2 D}(t, r)=\frac{\sqrt{2 \pi}}{4 t D} e^{-\frac{r^{2}}{4 t D}}
$$

the 2D Green's function in $\mathrm{t}, \mathrm{r}$ variables.

The Green's function in case B can be written in a different useful form. The sideways heat flow can be replaced as $\Lambda k^{2} d \rightarrow \Lambda \frac{8 d}{w_{0}^{2}} \rightarrow \frac{C d}{\tau_{D}}$, representing the in-plane diffusion in the metal film with a characteristic time $\tau_{D}=\frac{w_{0}^{2}}{8 D}$ in the time domain. Then, $=\frac{1}{\frac{1}{\tau_{D}}+i \omega+\frac{1}{\tau_{G}}}=\frac{1}{\frac{1}{\tau_{e f f}}+i \omega}$, where $\frac{1}{\tau_{e f f}}=\frac{1}{\tau_{D}}+\frac{1}{\tau_{G}}$ is the total rate at which heat leaves the layer, either through in-plane heat diffusion or through the interface. This expression for $\mathbb{G}$ shows that we can expect the phase of $T_{\text {top }}(\omega)$ to change on transitions from one interface-, film-, or substrate dominated cooling regime to another.

Ballistic corrections are important when the heat carrier mean free path is comparable to or larger than the sample thickness. The hot electron lifetime depends on energy approximately as $t \propto 1 /\left(E-E_{F}\right)^{2}$ in crystalline $\mathrm{Cu}$ [164] and $\mathrm{Ag}$ [165]. As electrons flow down the dispersion curves, scattering inelastically with other electrons, phonons, and impurities, their lifetime t, group velocity $v=\partial E / \partial k$, and mean free path $l=v t$ vary and are difficult to measure or calculate. Near the Fermi energy, the electron mean free path can be estimated from electrical conductivity measurements and is on the order of 10-50 nm in pure, crystalline metals at room temperature [166]. We consider the ballistic corrections, as the thickness of our samples is the same order of magnitude. 

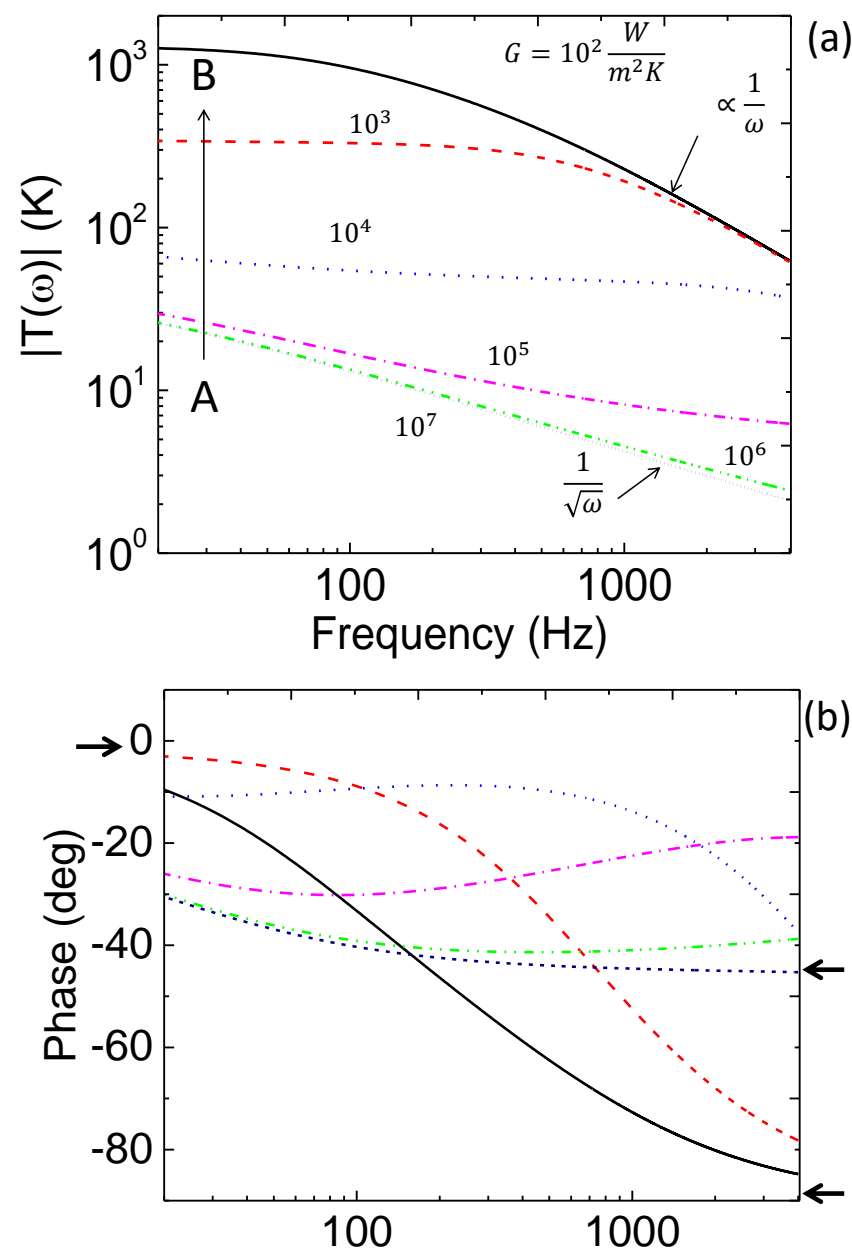

Frequency $(\mathrm{Hz})$

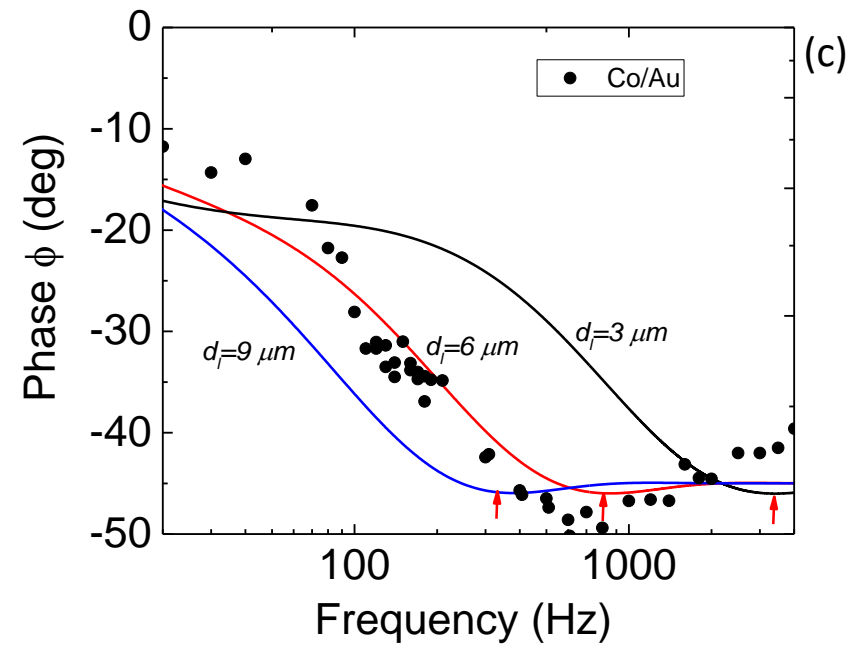

Figure 5.5 | Amplitude (a) and phase (b) of the temperature modulation for different G. As expected, the amplitude $|T|$ increases from A to B. The parameters are $P_{a b s}=$ $30 \mathrm{~mW}, d=30 \mathrm{~nm}, w_{e f f}=200 \mu \mathrm{m}, D_{s}=5 \times 10^{-7} \mathrm{~m}^{2} / \mathrm{s}, \Lambda_{s}=1 \mathrm{~W} / \mathrm{mK}, D_{f}=0.1 \times$ 
$10^{-4} \mathrm{~m}^{2} / \mathrm{s}, \Lambda_{f}=100 \mathrm{~W} / \mathrm{mK}$ (c) Results with a glass layer $d_{l}$ with $D_{l}=0.05 D_{s}, \Lambda_{l}=$ $0.05 \Lambda_{s}, w_{\text {eff }}=200 \mu \mathrm{m}$. Similar results are obtained for layers with the same $D_{l} / d_{l}^{2}$ ratio. The case of $d_{l}=6 \mu \mathrm{m}$ is shown for $G=10^{5} \mathrm{~W} /\left(\mathrm{m}^{2} \mathrm{~K}\right)$ (dashed) and $G \rightarrow \infty$ (solid red line).

Ballistic heat transfer is an active area of research. It predicts a reduced effective thermal conductivity in thin structures due to additional scattering of ballistic heat carriers at the interfaces. This reduction has been observed in, among others, across GaAs/AlAs superlattices [167], along silicon thin films [168-170] and nanowires [171], across $\mathrm{WSe}_{2}$ layers [172], along silicon layers with Ge dot structures [173], and along graphene layers [174]. A model was developed to calculate the effective conductivity along the superlattice structure, including the additional interface scattering [175]. An effective thermal conductivity along the structure could also be obtained with a boundary scattering function $F(\delta=d / l, p)$ that depends on the ratio of the film thickness $\mathrm{d}$ to heat carrier mean free path 1 and the specular reflection coefficient $p$ [176, 177], with ballistic corrections larger for smaller $\mathrm{p}$ or more diffuse scattering. The metallic value $\Lambda_{f}=$ $100 \mathrm{~W} / \mathrm{mK}$ we used for in-plane film conductivity may be considered to include ballistic corrections.

Similarly, ballistic corrections are made to heat transfer across interfaces. Heat transfer across metal/dielectric interfaces and the associated thermal boundary resistance were calculated with phonon-phonon and electron-phonon scattering [178-181]. Including interface scattering of ballistic phonons in the Boltzmann transport equation for a dielectric film between metal boundaries gave a heat flux $F=\Lambda_{e f f} \Delta T / d$ for small $\Delta T$, with an effective thermal conductivity $\Lambda_{\text {eff }}=\Lambda_{\text {bulk }} /(1+4 l / 3 d)<\Lambda_{\text {bulk }}[182]$. 
Ballistic corrections were also considered in detail for semiconductor interfaces with specular and diffuse, elastic and inelastic, scattering [183]. An increase of the effective thermal boundary resistance in the ballistic regime at metal/dielectric interfaces has been observed for nickel/fused silica and nickel/sapphire interfaces [184], in which a resistance ballistic correction $R_{B C}$ was added to the size independent thermal boundary resistance $R_{T B R}$ due to the different thermal properties of the dielectric and metal. The interface conductance in Eq. (3) may be viewed as an effective conductance $G=G_{\text {eff }}=$ $1 /\left(R_{T B R}+R_{B C}\right)$ that includes ballistic corrections. These results are applied to the two experimental configurations - the chopper modulation and the scanning beam.

\section{B. Temporal modulation}

In the pump-probe measurements, the signal is proportional to the film temperature [Eq. (1)], which can be calculated with Eq. (4).

One may expect to observe heat diffusion following case A because interfaces

between dense solids have $G \gg \Lambda_{s} q_{s} \sim 10^{5}(1+i) \frac{W}{m^{2} K}$ (Ref. 3) for typical $k_{e f f}$ and chopper frequencies f, or $T_{\text {top }} \propto \frac{1}{\sqrt{f}}$. Surprisingly, measurements show the $1 / \mathrm{f}$ dependence of two-dimensional heat flow above $200 \mathrm{~Hz}$ (Figure 2) for $\mathrm{Co} / \mathrm{Pd}$ and $\mathrm{Co} / \mathrm{Ag}$ and a dependence between $1 / \mathrm{f}$ and $1 / \sqrt{ } \mathrm{f}$ for $\mathrm{Co} / \mathrm{Au}$.

The k-integral of Eq. (1) has been calculated numerically for different interface conductances G. The evolution of $\mathrm{T}(\omega)$ amplitude and phase from case A to B is shown in Figs. 5(a) and 5(b). The main features of these plots can be understood qualitatively. In the limit of low frequency (steady-state), T( $\omega)$ is real and $\phi \rightarrow 0$. At large $\omega$, the Green's function dependence on $\mathrm{k}$ is negligible and the k-integral reduces to 
$\int_{0}^{\infty} d k k e^{-\frac{k^{2}}{8}\left(w_{0}^{2}+w_{1}^{2}\right)}=\frac{4}{w_{e f f}^{2}}$. In case A, we obtain $T(\omega) \propto \frac{1}{\sqrt{i \omega}}$ or a $1 / \sqrt{ }$ f dependence and a $-45^{\circ}$ phase at large $\omega$. Similarly, in case $B$, we obtain $T(\omega) \propto \frac{1}{i \omega}$ or a $1 /$ f and $-90^{\circ} 1$ phase at large $\omega$ (arrows).

The values of $\mathrm{G}$ required to obtain the observed 1/f dependence (Figure 2) are $\leq 10^{2} \frac{W}{m^{2} K}$, much lower than typical glass-metal conductances $>10^{7} \frac{W}{m^{2} K}$ (Ref. [185]) and on the order of the heat transfer coefficient for near-field radiative heat transfer between glass and Au interfaces at a $10 \mathrm{~nm}$ separation [186]. Such free-standing metal films would heat up to very high temperatures $\left[|T(\omega \rightarrow 0)|>10^{3} \mathrm{~K}\right.$ in Figure 5(a)] and be structurally unstable. The minimum in phase at $1000 \mathrm{~Hz}$ is also not obtained.

The simplest geometry of one metallic film is insufficient and considering a more complicated structure is necessary. A solution is to add a new layer 1 between the metal film and glass substrate [Figure 4(c)]. This layer may be the same porous birefringent layer, made in the glass substrate in the immediate vicinity of the absorbing film, and observed in polarizing microscopy. A glass layer of thickness $d_{l}$ and conductance to the glass substrate $G_{2} \gg \Lambda_{s} q_{s}$ will add one matrix $\left(\begin{array}{cc}\cosh \left(q_{l} d_{l}\right) & -\frac{1}{\Lambda_{l} d_{l}} \sinh \left(q_{l} d_{l}\right) \\ -\Lambda_{l} d_{l} \sinh \left(q_{l} d_{l}\right) & \cosh \left(q_{l} d_{l}\right)\end{array}\right)$ to Eq. (3), where the small thickness approximation $q_{l} d_{l} \ll 1$ has not been made.

The result for $T_{\text {top }}(\omega, \mathrm{k})$ is unwieldy, but numerical calculations can be made for different layer thickness dl. These show a characteristic minimum [arrows in Figure 5(c)], as the phase climbs to the semi-infinite substrate angle $-45^{\circ}$. This occurs near $=\frac{D}{\pi d_{l}^{2}}$, when the depth of thermal modulation is smaller than the layer thickness and the sample 
begins to resemble a semi-infinite substrate. The minimum in phase observed at $1000 \mathrm{~Hz}$ is obtained. The results in Figure 5(c) are weakly dependent on the metal/dielectric interface $G$ [unlike the case in Figure 5(b)] because the heat transfer across the structure is now dominated by the glass layer, approximately represented by an effective conductance $G_{1}=\Lambda_{l} / d_{l} \sim 10^{4} \mathrm{~W} /\left(\mathrm{m}^{2} \mathrm{~K}\right) \ll G$. Measurements for the Co/Au sample from Figure 2(b) have been shifted along the y-axis and added for comparison. The model explains the main experimental features.

\section{Demagnetization patterns}

Only the pump beam is present in scanning measurements and its pulse sequence is replaced with a continuous-wave beam as before. In contrast to the previous case, the cylindrical symmetry is lost and the initial temperature spectrum is not sharply defined for a moving beam. It is advantageous to work with $\mathrm{t}, \mathrm{r}$ variables.

A Gaussian beam moving at a velocity $v_{s}$ along the $\mathrm{x}$-axis is a heat source $F\left(t^{\prime}, x^{\prime}, y^{\prime}\right)=$ $\frac{2 P_{a b s}\left(t^{\prime}\right)}{\pi w_{0}^{2}} e^{-\frac{2\left(x^{\prime}-v_{s} t^{\prime}\right)^{2}}{w_{0}^{2}}-\frac{2\left(y^{\prime}\right)^{2}}{w_{0}^{2}}}$ giving an initial temperature $T\left(t^{\prime}, x^{\prime}, y^{\prime}\right)=\frac{F\left(t^{\prime}, x^{\prime}, y^{\prime}\right)}{C d}$, where the absorbed power depends on time $t^{\prime}$ because of the variable transmission observed on crossing stripes (Figure 2). The temperature at a later time $t>t^{\prime}$ is $T(t, x, y)=$ $\int d t^{\prime} d x^{\prime} d y^{\prime} \mathbb{G}\left(t-t^{\prime}, x-x^{\prime}, y-y^{\prime}\right) T\left(t^{\prime}, x^{\prime}, y^{\prime}\right)$. The solution with the 3D Green's function for a substrate with surface absorption has been obtained before [187]. For the 2D Green's function [Eq. (6)], the integration over $x^{\prime}$ and $y^{\prime}$ can be done by completing the square to give 


$$
T(t, x, y)=\frac{2 \sqrt{2 \pi}}{C d} \int_{-\infty}^{t} d t^{\prime} \frac{P_{a b s}\left(t^{\prime}\right)}{8 D\left(t-t^{\prime}\right)+w_{0}^{2}} e^{-2 \frac{\left(x-v_{S} t^{\prime}\right)^{2}+y^{2}}{8 D\left(t-t^{\prime}\right)+w_{0}^{2}}}
$$

This expression shows how in-plane diffusion [the $8 D\left(t-t^{\prime}\right)$ term] combines with the beam profile tails (the $w_{0}^{2}$ term) to give a $\mathrm{T}$ increase at $(x, y)$ when the laser beam center is at $\left(v_{s} t^{\prime}, 0\right)$.

As expected, this integral is divergent for a stationary beam of constant intensity $\left[x=0, y=0, P_{a b s}\left(t^{\prime}\right)=\right.$ const. $]$. In contrast to a 3D substrate, a thermally $2 \mathrm{D}$ film does not cool well under a steady heat flux. To remove this unphysical divergence, the heat flow conditions must be changed from strictly two-dimensional. The Green's function for a layer with surface absorption and an infinite interface conductance $G \rightarrow \infty$ to the substrate can be reduced to a double integral [188]. A different approach can be taken for an interface with a finite conductance $\mathrm{G}$, by allowing an additional heat transfer channel through the interface, as done in Sec. 5.4 A, or

$$
\mathbb{G}=\mathbb{G}_{2 D}\left(t-t^{\prime}, x-x^{\prime}, y-y^{\prime}\right) e^{-\frac{t-t^{\prime}}{\tau_{G}}}
$$

This removes the temperature divergence. Results of the maximum film temperature $T_{\max }(x, y)$ obtained during the laser scan for a variable $P_{a b s}$, modulated as shown in Figure 2 give a sequence of peaks [Figure 6(a), left panel]. Dividing into two cases [white and black, Figure 6(a), right panel], above and below a borderline temperature gives plots that correspond well with the experimental observations (Figure 3). In particular, the decreasing spot size and re-emergence of the stripe on crossing the stack is obtained. A narrowing of the features, calculated for a moving beam [Figure 6(b)], is consistent with observations [Figure 3(c)]. 


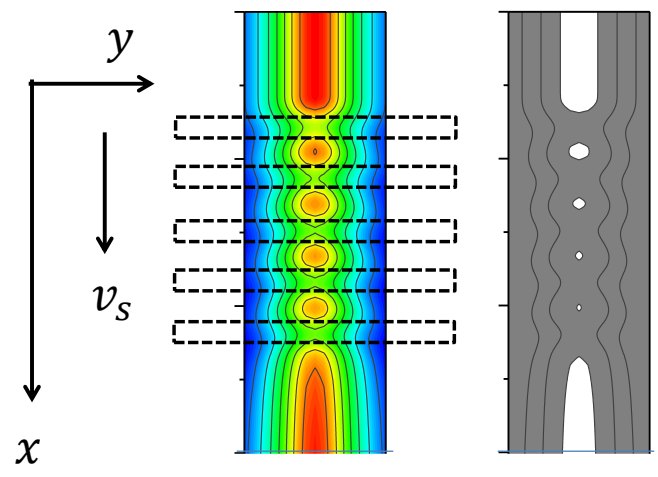

(a)

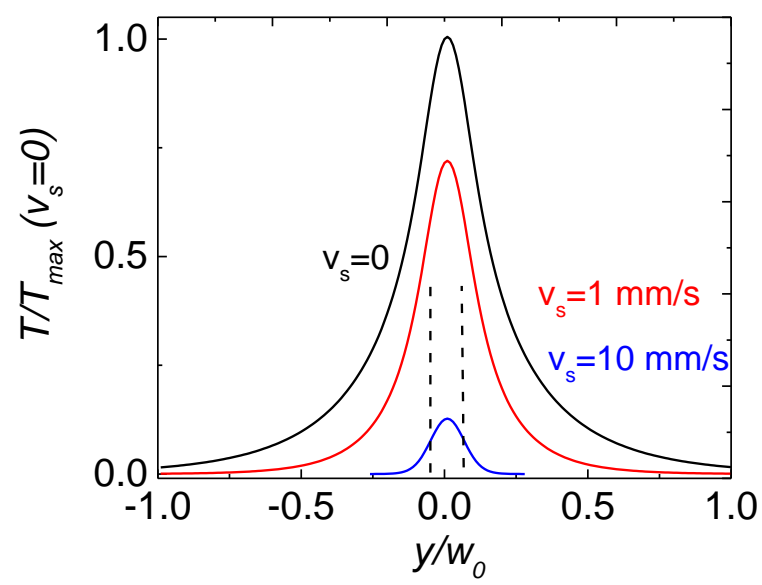

(b)

(c)

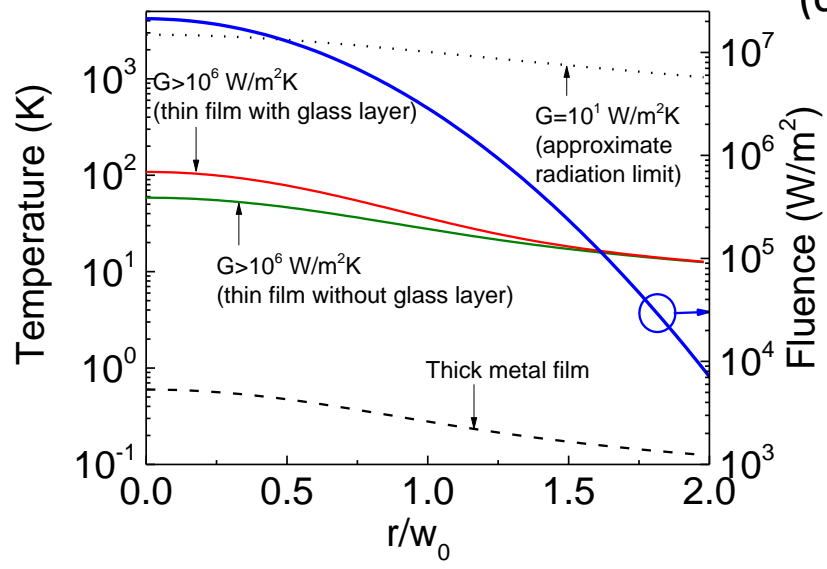

Figure $5.6 \mid$ (a) Film $\mathrm{T}_{\max }$ during a scan with power modulated across the horizontal stripes (dashed features). (b) The steady-state solution at $v_{s}=0$ compared to a profile induced by a beam moving with $v_{s}=1.10 \mathrm{~mm} / \mathrm{s}$ shows the larger radius at the end of the scan. (c) Steady-state temperature radial temperature profiles for interface conductances G shown and $P_{a b s}=30 \mathrm{~mW}, d=30 \mathrm{~nm}, w_{0}=200 \mu \mathrm{m}, D_{\mathrm{s}}=5 \times 10^{-7} \mathrm{~m}^{2} / \mathrm{s}, \Lambda_{s}=$ $1 \mathrm{~W} / \mathrm{mK}, D_{f}=0.1 \times 10^{-4} \mathrm{~m}^{2} / \mathrm{s}, \Lambda_{f}=100 \mathrm{~W} / \mathrm{mK}$ without the glass layer, 
compared to the 3D case (thick metal film), and with results including the same glass layer as in figure 5(c). The difference between results for $G=10^{6} \mathrm{~W} /\left(\mathrm{m}^{2} \mathrm{~K}\right)$ and $G \rightarrow \infty$ is negligible. The right axis shows the Gaussian beam fluence profile.

The cylindrical symmetry is restored in the stationary condition $\left(v_{s}=0\right)$ and this case can be applied to illustrate the temperature increase due to heat accumulation. In the substrate limit (case A with $d \rightarrow 0$ ), a Hankel transform of $T_{\text {top }}(\omega=0, k)=\frac{F_{t o p}(\omega=0, k)}{\Lambda_{s} q_{s}}$ gives the known solution $T_{t o p}(\omega=0, r)=\frac{1}{\sqrt{2 \pi}} \frac{P_{a b s}}{\Lambda_{s} w_{0}} e^{-\frac{r^{2}}{w_{0}^{2}}} I_{0}\left(\frac{r^{2}}{w_{0}^{2}}\right)$, where $\mathrm{I}_{0}$ is the modified Bessel function [65][189]. Then, $T_{\max }=\frac{P_{a b s}}{\sqrt{2 \pi} \Lambda w_{0}} \approx \frac{30 \mathrm{~mW}}{2.5 \times 100 \frac{\mathrm{W}}{\mathrm{mK}} 200 \mu \mathrm{m}} \sim 0.6 \mathrm{~K}$ for typical metallic thermal conductivity K. Heat dissipates quickly between pulses and $T_{a c c}$ can be neglected in thick, thermally conducting samples.

In contrast, our superlattice samples are thermally thin. In general, an interface at a depth smaller than the thermal modulation depth $L_{t h}=\sqrt{\frac{D}{\pi f}}$ will affect heat diffusion, where $L_{t h}=30 \mu m \gg d$ for $f=3 \times 10^{3} \mathrm{~Hz}$ and a good thermal conductor with $D=0.1 \times 10^{-4} \mathrm{~m}^{2} / \mathrm{s}$. Temperature profiles calculated with a Hankel transform of Eq. (4) are significantly higher as the large heat fluxes possible through a semi-infinite substrate are reduced [Figure 6(c)]. The combination of large light absorption in the metallic film and small glass thermal conductivity can raise $T_{a c c}$ above that of a thick metallic film. Heat accumulation and large temperature gradients in our samples explain the observed forces on magnetic domain walls during all-optical switching [150] and how a final demagnetized state can be obtained from both heat accumulation $T_{a c c}$ and transient $T_{t r}$ ultrafast demagnetization [155]. 
A large $T_{a c c}$ in magnetic materials can be beneficial (for instance, in heat-assisted magnetic recording) or undesired if measurements at low temperature are required. Heatassisted magnetic recording heats up a sample to temperatures close to the Curie temperature, where the coercive field is reduced. This makes it possible to use materials with a high magnetic anisotropy and coercive fields at room temperature. The stronger magnetic order allows patterning the materials into smaller structures that would otherwise be superparamagnetic at room temperature and unusable for magnetic recording. The heating can be done with near-field optics, allowing in principle perpendicular magnetic recording densities up to 1-100 $\mathrm{Tb} / \mathrm{in}$ [126] for structured bit media [190], much larger than the $10 \mathrm{~Gb} /$ in [126] densities of longitudinal magnetic recording [191]. This work on magnetic superlattices, one of which $(\mathrm{Co} / \mathrm{Pd})$ has perpendicular magnetic anisotropy, illustrates the heating that can be obtained in this approach for un-patterned media. Future work may examine the smaller heat accumulation predicted in one-dimensional structures at the same average fluence with increased repetition rates, heat accumulation in dots, offset pump-probe beams or conditions with a larger $k_{e f f}$ from tighter focusing.

\subsection{CONCLUSION}

Heat diffusion in metallic superlattices on glass substrates has been examined with pump-probe measurements and polarizing microscopy of laser-induced demagnetization patterns. Green's function solutions of the heat diffusion equation quantify the temperature in the two experimental configurations. A glass layer is required to explain the temporal modulation frequency dependence and demagnetization patterns 
are reproduced with an interface conductance. Thermo-transmittance measurements can be applied in examining heat accumulation and diffusion in thin samples on thermally insulating substrates under an intense light field and in characterization of a multilayer device thermal response. 


\section{CHAPTER 6: OTHER MEASUREMENTS}

\subsection{Temperature dependence of the coercive field in GdFeCo films}

The total magnetic moment of a ferrimagnetic compound is the sum of the magnetic moments of the two components pointing in opposite directions. Figure 6.1 shows the temperature dependence of magnetization of RE and TM elements. The total magnetic moment $M_{\text {total }}=\left|M_{R E}-M_{T M}\right|$ reaches a minimum at the compensation temperature $\mathrm{T}_{\text {comp }}$.

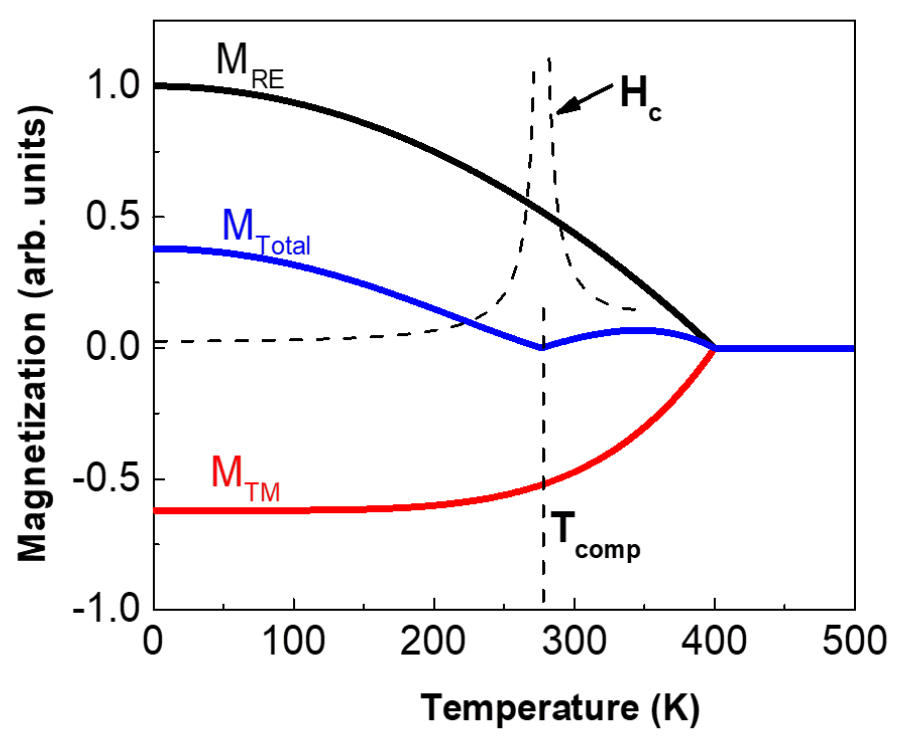

Figure 6.1 | Variation of $\mathrm{M}_{\mathrm{RE}}, \mathrm{M}_{\mathrm{TM}}, \mathrm{M}_{\text {total }}$ and coercive field $\mathrm{H}_{\mathrm{c}}$ with temperature.

Using the variable temperature MOKE setup, we investigated the hysteresis behavior of $\mathrm{Gd}_{x} \mathrm{Fe}_{1-x-y} \mathrm{Co}_{y}$ (GFC) samples in polar and longitudinal geometries. 
Samples of uniform thickness were attached to the cold finger of a cryostat in the vacuum chamber of the MOKE setup. Measurement in L-MOKE and P-MOKE geometries showed the sample magnetization is in-plane, as no loop opening was observed in PMOKE. Changing the temperature in a warming cycle in L-MOKE measurements revealed a decrease in coercive field with increasing temperature (Figure 6.2). This is consistent with the temperature dependence of the coercive field in rare-earth transitionmetal (RE-TM) compounds and denotes we are measuring the right-hand side of the $H_{c}(T)$ curve (Figure 6.1). Therefore, the compensation temperature $T_{\text {comp }}<100 \mathrm{~K}$, which corresponds to a Gd doping of $x<22 \%$ in the sample [192].

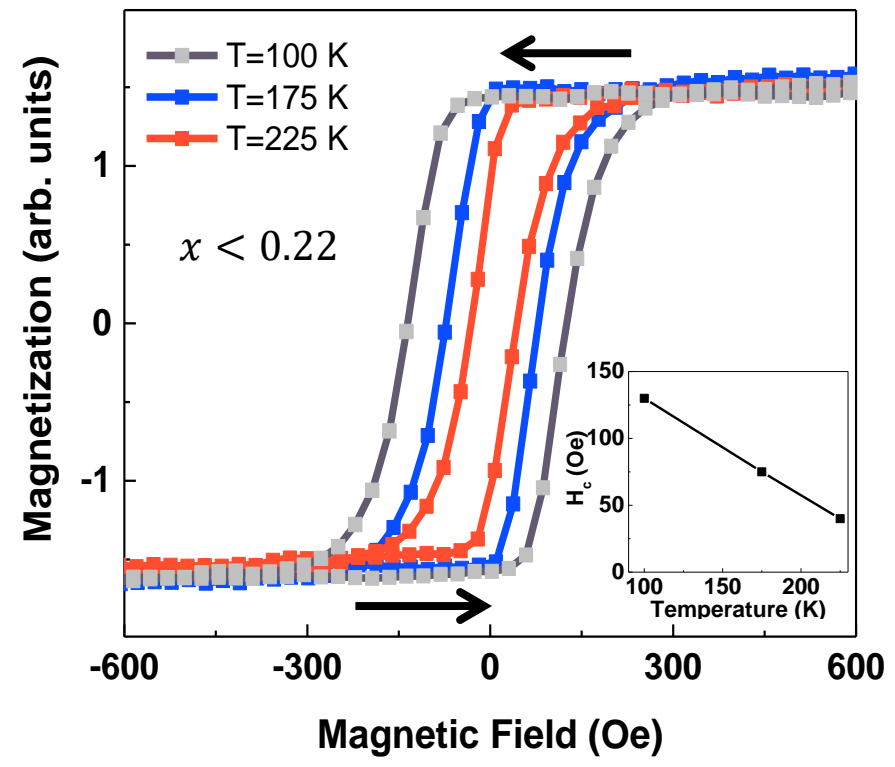

Figure $6.2 \mid$ Decrease of the coercive field $H_{c}$ from $130 \mathrm{Oe}$ at $100 \mathrm{~K}$ to $40 \mathrm{Oe}$ at $225 \mathrm{~K}$.

\subsection{Detecting the magnetization reorientation with doping}

MOKE also provides information on the direction of magnetic moment. For instance, in-plane (out-of-plane) magnetization corresponds to a square-shaped loop in LMOKE (P-MOKE) geometry (see next section). 
Measurements on a GFC sample with thickness variation showed two typical loops depending on the position on sample (Figure 6.3). In a normal hysteresis loop, the magnetization with increasing $H_{\text {ext }}$ is smaller than magnetization with decreasing $H_{\text {ext }}$. While at one area the hysteresis loops looked normal (Figure 6.3.a), in other areas we observed inverted hysteresis loops (Figure 6.3.b), or the magnetization with increasing $H_{\text {ext }}$ is higher than the one with decreasing $H_{\text {ext }}$.

The result can be explained based on a variable doping. The total magnetization $M_{\text {total }}=\left|M_{R E}-M_{T M}\right|$ points in same direction as the external magnetic field. Since a MOKE measurement with visible light is mainly sensitive to the magnetization of the TM component, the detected magnetization corresponds to $M_{T M}$ and reversal of the $\mathrm{M}_{\mathrm{TM}}$ direction results in a reversal of the MOKE hysteresis loop. The magnitude of RE and TM magnetizations change as a function of doping. If the dominating element is TM, then the MOKE magnetization increases with magnetic field and we get a normal hysteresis loop. However, if the RE magnetization dominates, then this would be the one which will point in the direction of the external field. Therefore, the TM magnetization will point in the direction opposite to the external magnetic field and MOKE hysteresis loops will be inverted. 


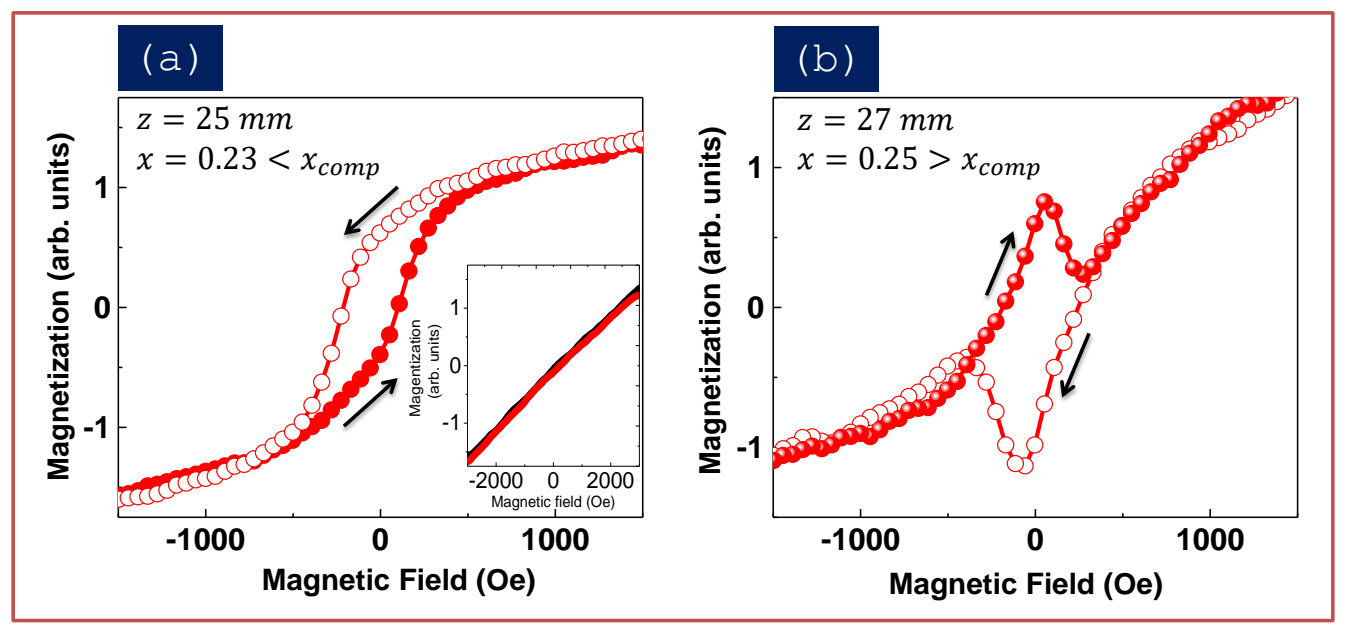

Figure 6.3 | L-MOKE measurements performed on the GFC sample with variable thickness showed two typical hysteresis loops. A normal (a) and an inverted (b) hysteresis loop. The inset in (a) shows the P-MOKE result.

\subsection{Finding the optimal thickness for PMA}

As mentioned earlier, the film thickness plays a crucial role in overcoming the shape anisotropy [193] and obtaining perpendicular magnetic anisotropy (PMA). Thicker films tend to have an in-plane easy axis. Decreasing the thickness may result in the emergence of an out-of-plane magnetization with the easy axis rotated to perpendicular direction with respect to the sample surface. The orientation of easy axis can be determined by MOKE measurements in longitudinal and polar geometries. A squareshape loop with well-defined saturation magnetization implies an easy-axis in the direction of applied field. If such loop is obtained in L-MOKE (P-MOKE) then the easy axis is in-plane (out-of-plane).

Depositing samples of variable thicknesses facilitated finding the optimal thickness for out-of-plane magnetization or PMA. We did MOKE measurements in both polar and longitudinal modes to identify PMA conditions for a sputter-deposited $[\mathrm{Co} / \mathrm{Pd}]_{4}$ 
sample of variable thickness. Figure 6.4 shows the hysteresis curves obtained at different thicknesses. Measurement in L-MOKE on the thick end of the sample showed a square shape loop (Figure 6.4.a) pointing to an in-plane magnetization. In addition, P-MOKE measurement at the same spot resulted in a tilted line with no opening (not shown), which confirms the L-MOKE result. As the sample was scanned toward thinner areas, an opening started to show up in P-MOKE (Figure 6.4.b), which corresponds to out-of-plane magnetization. Further decreases in thickness resulted in smaller loops (Figure 6.4.c shows one example) and eventually, its disappearance at the thin end of the sample (not shown).

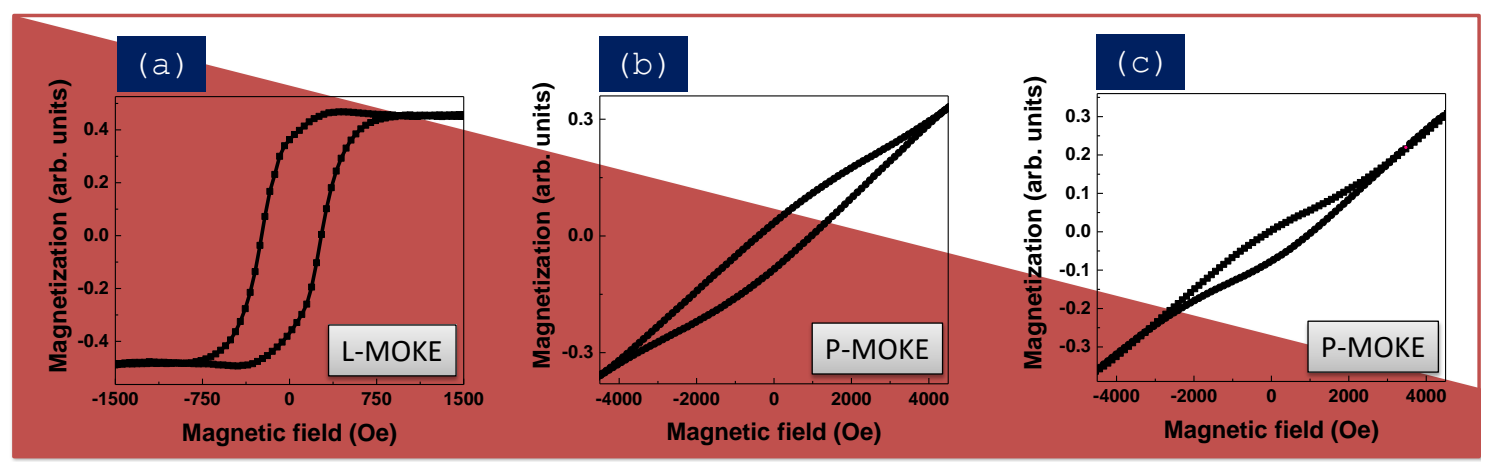

Figure 6.4 | MOKE measurements performed on the sputter deposited $[\mathrm{Co} / \mathrm{Pd}]_{4}$ sample with variable thickness in (a) Longitudinal and (b) \& (c) Polar modes. The red wedge represents the thickness variation in the sample. The loop in (a) was obtained at the thickest end of the sample, (b) and (c) were obtained at two different spots on thinner parts of the sample with out of plane magnetization.

The results obtained in polar geometry were consistent with L-MOKE measurements at the same spots of the sample. P-MOKE curves showed an opening for a small thickness-range. This implied a rotation of easy axis from in-plane to out-of-plane direction as the thickness decreased. Knowing the optimal thickness permitted the 
quantitative determination of the appropriate deposition parameters necessary to obtain a uniform sample with perpendicular magnetic anisotropy.

Thickness dependence of easy axis orientation was observed in other samples as well. For instance, a $\mathrm{Co}_{2} \mathrm{FeAl}(\mathrm{CFA})$ sample of $36 \mathrm{~nm}$ thickness, sputter-deposited on Si(111) substrate, exhibits an in-plane easy-axis, which is not the case for a sample of 18 nm thickness (Figure 6.5). However, the analysis of the hysteresis loops in CFA is more complex because the material is biaxial, with three different magnetic axes.
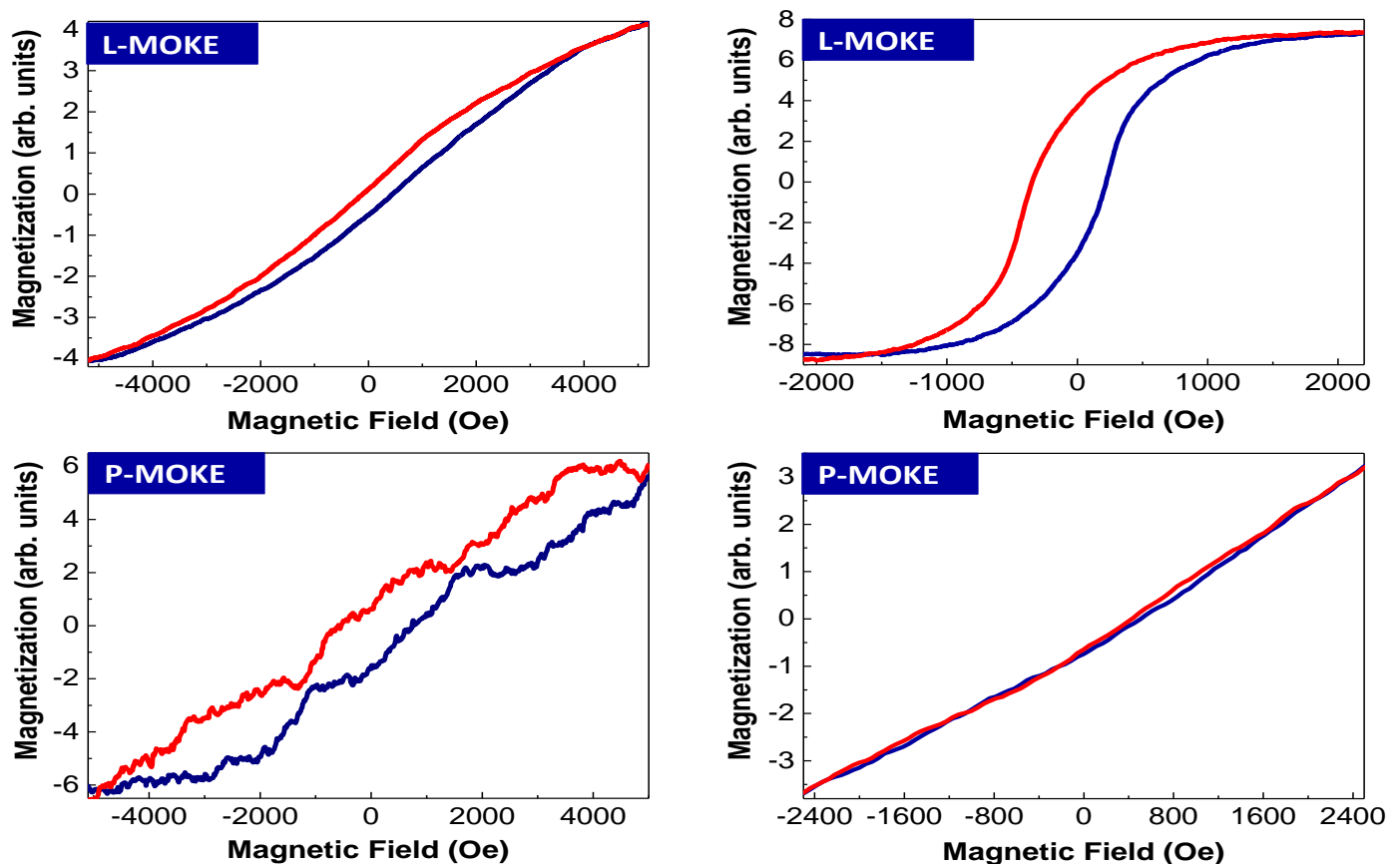

Figure 6.5 | Hysteresis loops measured on CFA/Si(111) samples of 18nm (left) and 36nm (right) thicknesses in longitudinal and polar geometries. The result illustrates an in-plane easy axis in the thicker sample. The hysteresis loops of the thinner film do not match an in-plane or out-of-plane easy axis and the magnetization may be at an angle. Since CFA is biaxial with three different magnetic axes, the analysis of the hysteresis behavior is not as straightforward as in a uniaxial material.

We also used MOKE to observe different in-plane magnetization directions and in-plane magnetic anisotropy. In this method, the magnetization is measured in an L- 
MOKE setup at constant field as the sample is rotated azimuthally. We used this technique to examine the in-plane magnetic anisotropy in $\mathrm{Co}_{2} \mathrm{Fe} A l$ (CFA) films sputter deposited on glass and $\operatorname{Si}(111)$ substrates. Figure 6.6 shows an example of such measurements on a $\mathrm{CFA} / \mathrm{Si}(111)$ sample, which implies a biaxial anisotropy.

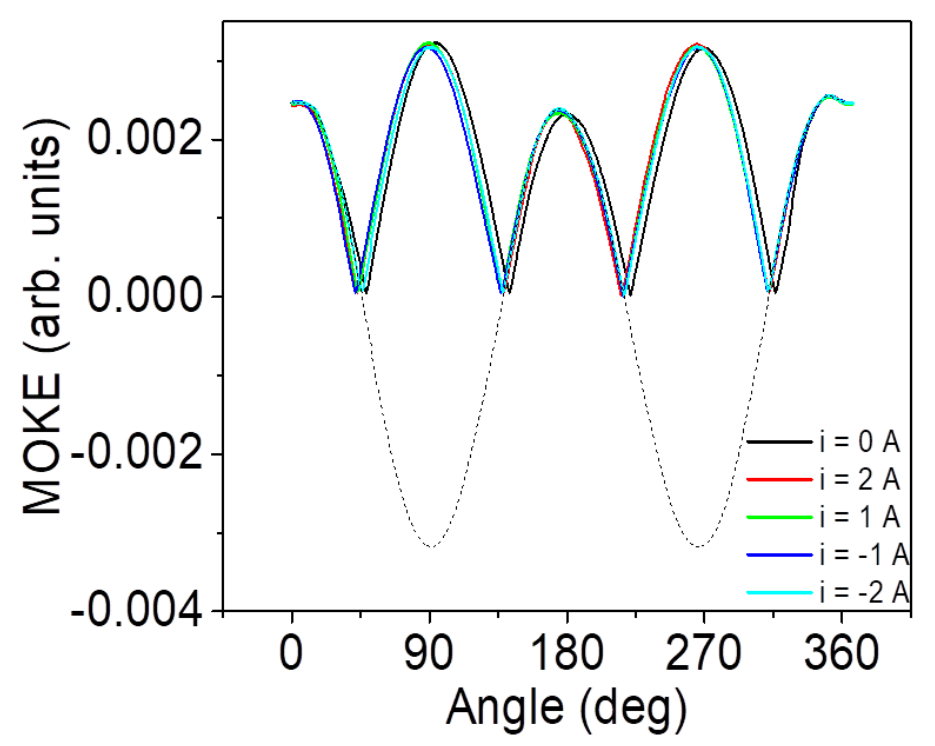

Figure 6.6 | Magnetization acquired at constant applied magnetic field. The sample was rotated 360 degree during this measurement. Different colors represent different field magnitudes. The $50 \mathrm{~nm}$ CFA sample was sputter deposited on a $\mathrm{Si}(111)$ substrate.

\subsection{Measuring the signal derivative}

In general, detecting the signal derivative can enhance small variations in the signal. However, there can be more benefits in using this method. As stated earlier, the magneto-optical pump-probe experiment is applied to measure the variation of temperature and magnetization as a function of delay time. Thermal effects contribute significantly to the data when using a high-repetition-rate laser. Therefore, to study the magnetization dynamics in more detail we need to enhance the magnetic signal. 
Measuring the derivative of the signal removes delay-independent thermal contribution to the data. In addition, minimizing the fluence variations increases the sensitivity to magnetization variations. For instance, this method can be applied for measuring small variations in coercive field and saturation magnetization at different pump-probe delays. Moreover, it allows a more precise measurement of the demagnetization $\tau_{M}$ and equilibration $\tau_{E}$ time.

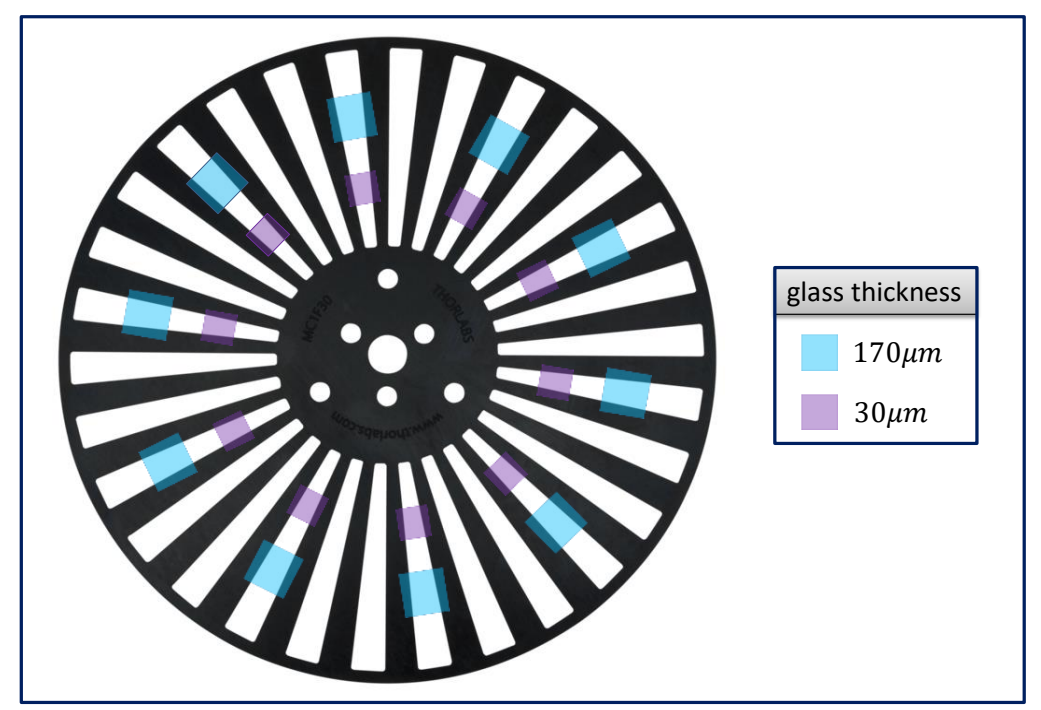

Figure 6.7 Illustration of a chopper wheel modified to create sensitivity to signal derivatives, with glass pieces attached to introduce an additional delay in the probe beam path. Then, measuring the signal at the glass frequency shows variations as the glass pieces modulate the delay, analogous to measuring the signal derivative $\Delta I / \Delta \tau$.

We developed a method to measure the derivative of the signal experimentally with glass squares added to the chopper wheel. Inserting glass in the beam path, introduces an additional delay between pump and probe beams (Figure 6.7). If the glass squares are distributed in a certain way, then the obtained signal will be $\frac{\Delta I}{\Delta \tau}$ instead of $I$, where $I$ is the light intensity and $\tau$ is the delay. In such an arrangement, the beam experiences two interruptions: one by glass and one by the metal chopper. It is similar to 
simultaneous use of two chopper wheels rotating at different frequencies $v$ (glass) and $v^{\prime}$ (metal).

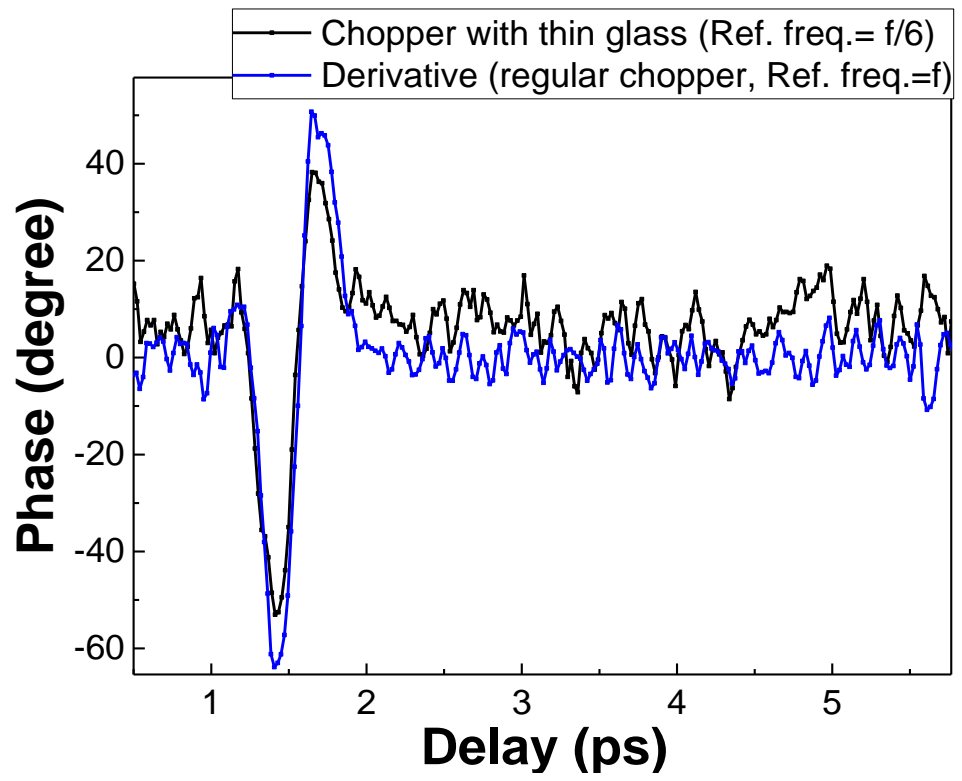

Figure 6.8 | Using the delay chopper is equivalent to taking derivative of the standard signal. This measurement was performed on a $[\mathrm{Co} / \mathrm{Pd}]_{4}$ sample with a $350 \mathrm{~mW}$ pump beam.

We tested our method by comparing the signals obtained with the standard and the glass chopper. The signal obtained with glass chopper overlaps the derivative of the signal measured without glass pieces (Figure 6.8). In this example, 10 glass squares were attached to a chopper wheel with 60 blades. Therefore, if we choose "f" $\mathrm{Hz}$ at the chopper controller, the glasses modulate the beam at a frequency of "f/6". Setting the lock-in reference frequency to "f" or " $\mathrm{f} / 6$ " determines whether the "regular" signal or its derivative is being measured. In addition, the resolution is directly proportional to the glass thickness. For instance, replacing $170 \mu \mathrm{m}$ with $30 \mu \mathrm{m}$ glass sheets improved the measurement resolution. This method may be applied for measuring thermal properties of semiconductor two-dimensional structures [194-199]. 


\subsection{High-power laser features}

Various other features were made when writing on the sample at high laser powers, as we were optimizing the fluence. Later, we applied much smaller powers to avoid structural changes or damage to the sample. Such features have been studied extensively [200].

Figures 6.9-6.13 show examples from our work.

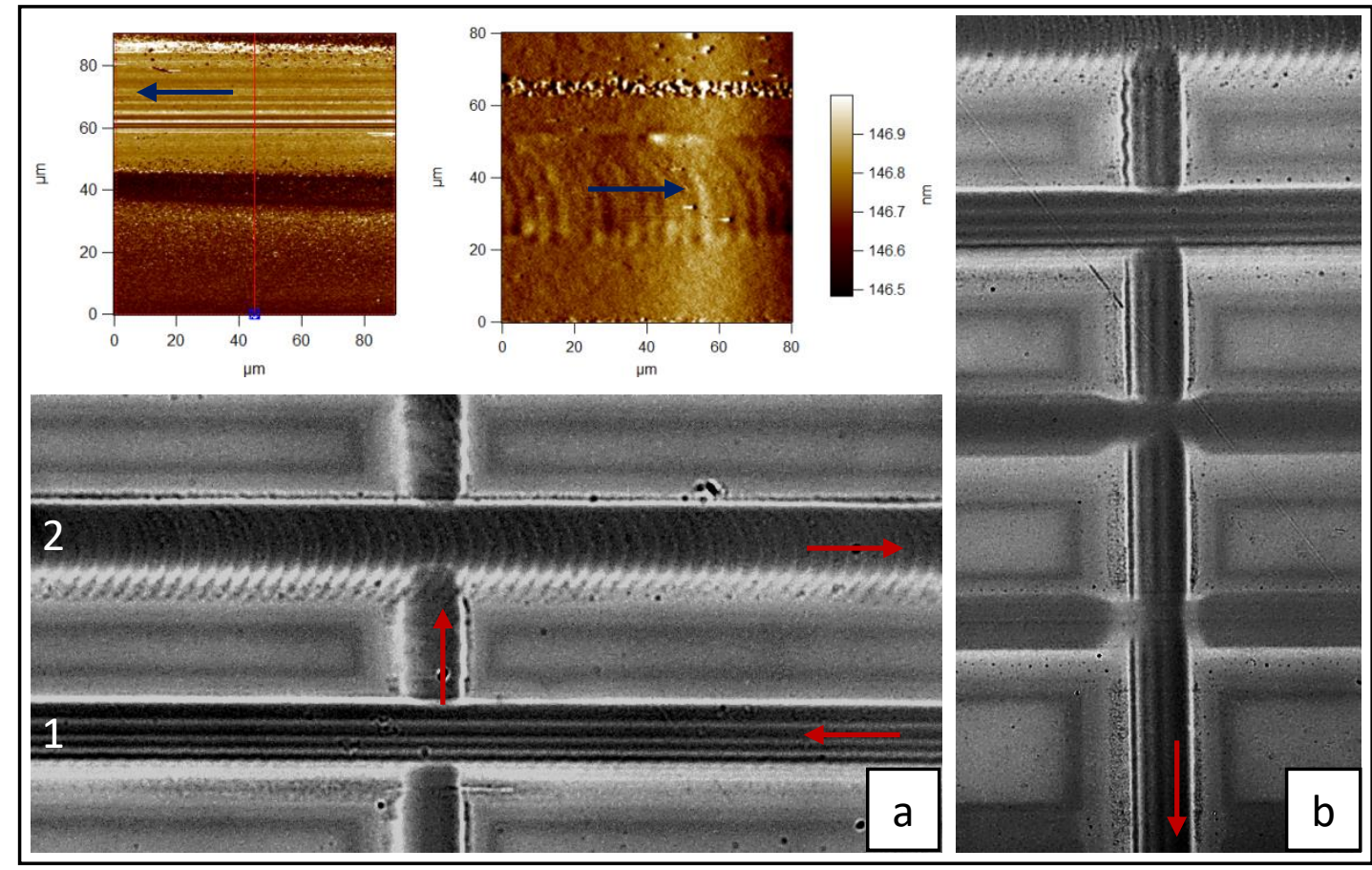

Figure 6.9 | Structures created during writing with high power laser on $6 \mathrm{~nm}$ thick $\mathrm{CoPd} /$ glass. The orientation of ripples/rims depends on writing direction (arrows). Features are visible in both AFM (color) and polarizing microscopy (gray scale) images (of different stripes) (a) The laser power employed for writing the $1^{\text {st }}$ stripe was $700 \mathrm{~mW}$ at $v=2.5 \mathrm{~mm} / \mathrm{s} .2^{\text {nd }}$ stripe is written with $\mathrm{P}=800 \mathrm{~mW}$ at $v=1 \mathrm{~mm} / \mathrm{s}$. For the vertical stripe (a) $\mathrm{P}=600 \mathrm{~mW}$ and $v=2.8 \mathrm{~mm} / \mathrm{s}$ and (b) $\mathrm{P}=700 \mathrm{~mW}$ at $v=2.8 \mathrm{~mm} / \mathrm{s}$. 


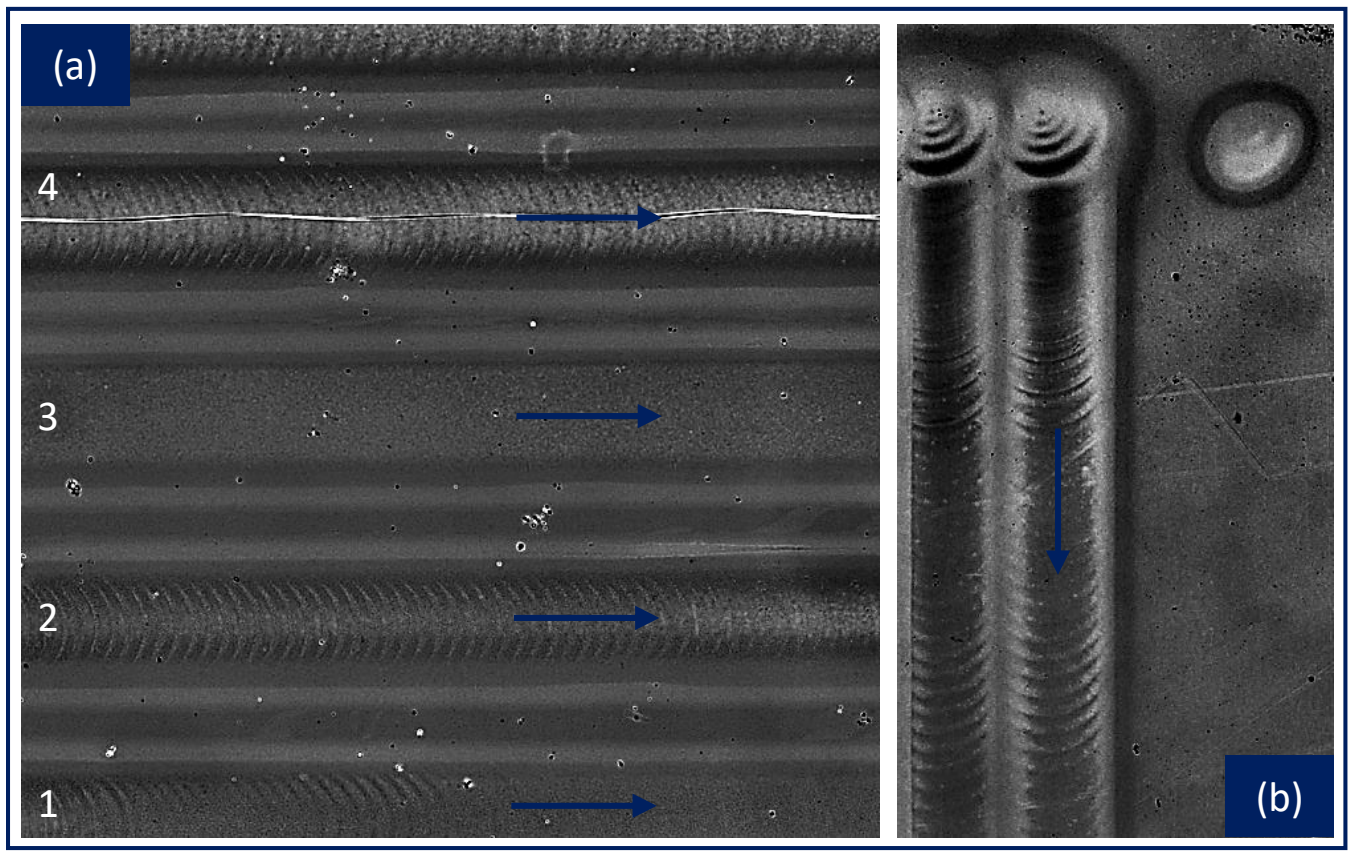

Figure 6.10 | Stripes written at different laser powers on $4 \mathrm{~nm}$-thick CoPd/glass. Arrows show the writing direction. (a) The writing speed increases slightly along the stripe and less heat is deposited. First stripe shows no ripples at the right side. Third stripe is written at low power and did not create ripples. (b) More pulses and larger absorbed heat created larger ripples at the beginning of the stripes (top): $\mathrm{P}=620 \mathrm{~mW}$ at $v=10 \mathrm{~mm} / \mathrm{s}$. 


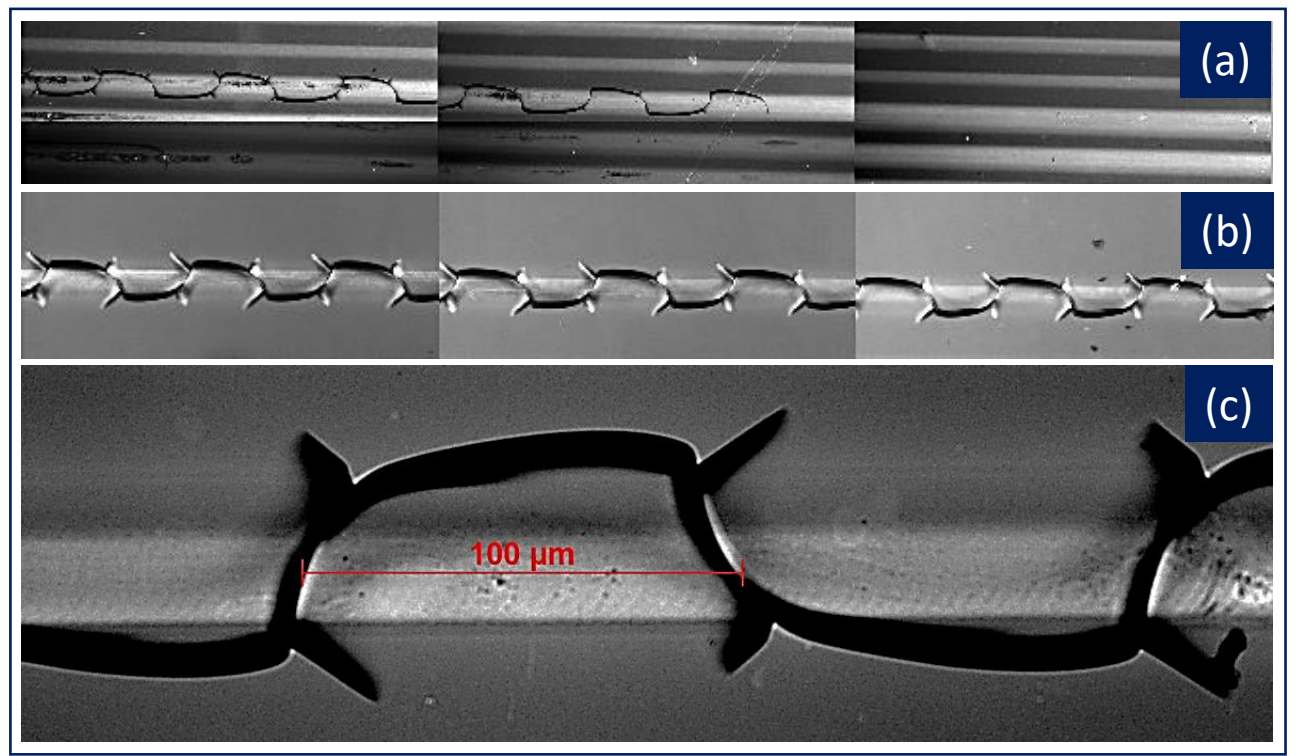

Figure 6.11 | Periodic features created during writing at high power on CoPd/glass of variable thickness. (a) High power features are more pronounced on the left side of image, as the film was thicker on this side, leading to higher heat absorption and more damage. (b) The stripe is written at $1000 \mathrm{~mW}$ and is present along most of the sample. (c) The same stripe at higher resolution. The periodicity of the features depends on laser power and writing speed.

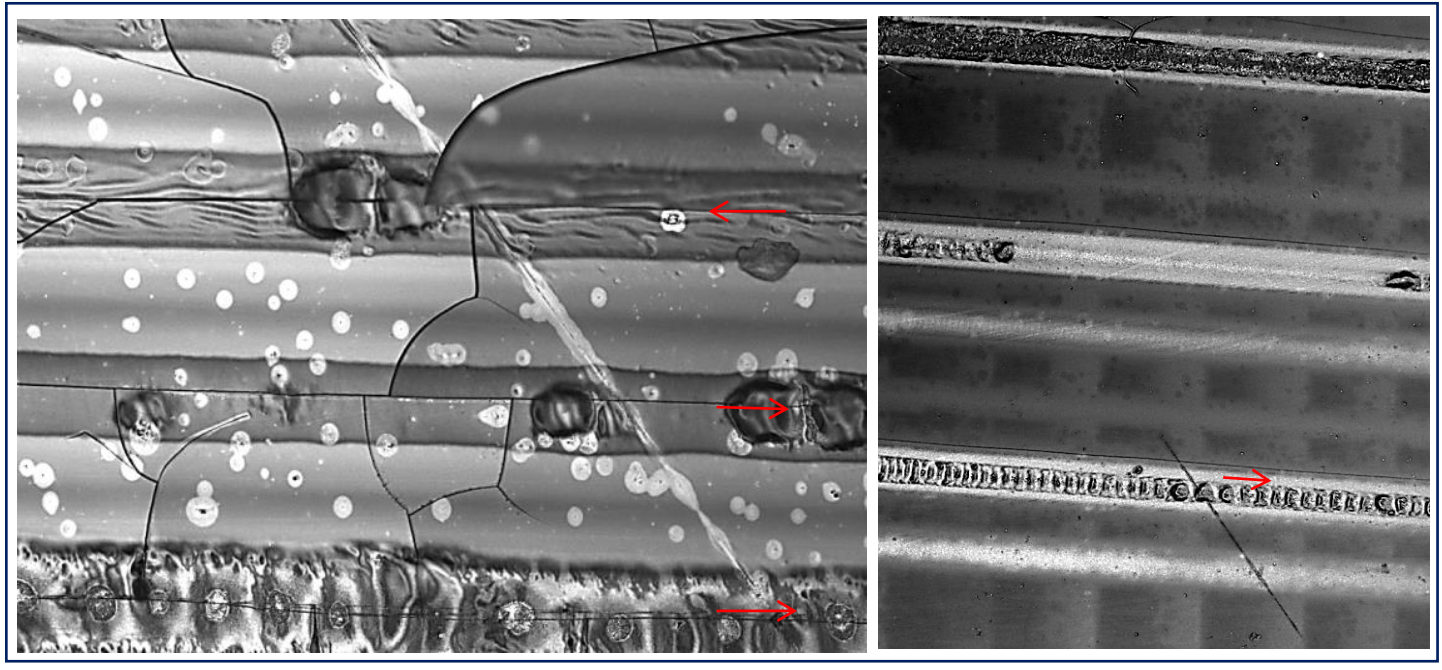

Figure 6.12 | Writing at different laser power on $\mathrm{CoPd} / \mathrm{glass}$ of variable thickness. 


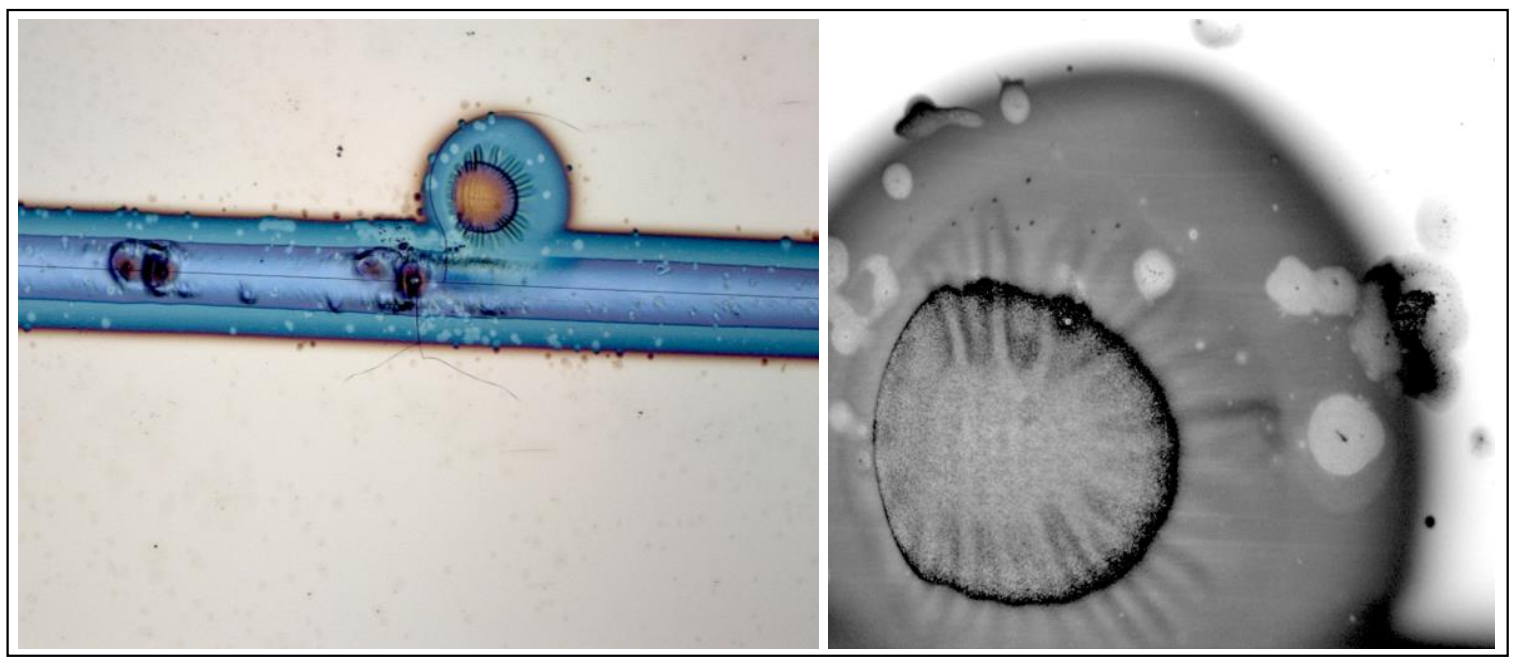

Figure 6.13 | Polarizing microscope image in reflection mode of a stripe and dot in $\mathrm{CoPd} /$ glass sample of variable thickness, showing details of the pattern created in the glass. 


\section{CHAPTER 7: SUMMARY AND OUTLOOK}

We presented our results on AOS in $\mathrm{Co} / \mathrm{Pd}$ superlattices, and discussed the role of temperature gradients and heat diffusion. Heat accumulation, due to the high repetitionrate laser, contributes to the switching process. Numerical calculations of heat diffusion are consistent with the observed domain expansion.

A pump-probe setup was used to measure the time dependence of ultrafast demagnetization. We presented the measurements, highlighting the importance of separating the magnetic and non-magnetic components of the signal. Micromagnetic simulations were applied to examine the role of demagnetizing fields in the timeevolution of a demagnetized cylinder into a switched state in a sample with perpendicular magnetic anisotropy. We discussed the relation between the simulation switched state and AOS in ferromagnetic materials.

Polarizing microscopy and pump-probe experiments were also applied to investigate heat diffusion in different ferromagnetic superlattices on glass substrates. We measured the frequency dependence of temperature modulations. A heat diffusion model including the laser-induced birefringent layer in the glass was applied to explain the data.

Measurements that did not belong to the three main results (chapters 3-5) close the dissertation. 
The imaging in this work was performed in the cleanroom with a Zeiss polarizing microscope. We are midway in building a Faraday microscope in our lab, which would enable us to image the sample in real time as we scan it under the Ti:S laser beam. The setup includes a high-performance CCD camera. We should be able to obtain good resolution images once the alignment is improved.

One method to increase the magnetic signal in magneto-optical pump-probe measurements is with the glass chopper. Another is to modulate both beams. To obtain the double modulation in our setup, we plan to introduce a photoelastic modulator (PEM) in the probe beam path, in addition to chopper modulation of the pump beam. The PEM frequency will be fed to the lock-in amplifier connected to the detector. The output of this lock-in amplifier should go to a second one, the reference frequency of which corresponds to the chopper in the pump beam path. In addition, we will explore measuring in reflection mode geometry. Measurements of semi-transparent $\mathrm{Co} / \mathrm{Pd}$ samples were done mostly in transmission mode. However, a recent experiment in reflection mode showed that, even though the signal is weak, the absence of the transient thermal peak may result in a better magnetic signal.

The work demonstrated the importance of accounting for temperature variations in UDM and AOS experiments due to heat accumulation, in particular when the structures are thin, the substrate thermally insulating, and the repetition rate large. This temperature increase can be put to good use, as it enables extending the temperature range of an optical measurement to temperatures above the Curie temperatures. We will apply the large and well-defined temperature variations in our samples to develop and verify experimentally a thermodynamics model of light-induced transformations of 
magnetic materials during UDM. Freestanding flexible substrates will be used to simplify the thermal analysis.

Other future research directions that will be pursued include thermal propagation and thermopower experiments in the ballistic regime in metallic two-dimensional structures and searching for AOS in antiferromagnetic complex oxides. 


\section{REFERENCES}

1. Beaurepaire, E., J.C. Merle, A. Daunois, and J.Y. Bigot, Ultrafast Spin Dynamics in Ferromagnetic Nickel. Physical Review Letters, 1996. 76(22): p. 4250-4253, https://link.aps.org/doi/10.1103/PhysRevLett.76.4250 https://journals.aps.org/prl/abstract/10.1103/PhysRevLett.76.4250

2. Tudosa, I., C. Stamm, A.B. Kashuba, F. King, H.C. Siegmann, J. Stöhr, G. Ju, B. Lu, and D. Weller, The ultimate speed of magnetic switching in granular recording media. Nature, 2004. 428: p. 831, 10.1038/nature02438 https://www.nature.com/articles/nature02438\#supplementary-information.

3. Choi, G.M., A. Schleife, and D.G. Cahill, Optical-helicity-driven magnetization dynamics in metallic ferromagnets. Nat Commun, 2017. 8: p. 15085, 10.1038/ncomms15085.

4. Koopmans, B., Laser-Induced Magnetization Dynamics, in Spin Dynamics in Confined Magnetic Structures II, B. Hillebrands and K. Ounadjela, Editors. 2003, Springer Berlin Heidelberg: Berlin, Heidelberg. p. 256-323.

5. Beaurepaire, E., G.M. Turner, S.M. Harrel, M.C. Beard, J.Y. Bigot, and C.A. Schmuttenmaer, Coherent terahertz emission from ferromagnetic films excited by femtosecond laser pulses. Applied Physics Letters, 2004. 84(18): p. 3465-3467, 10.1063/1.1737467.

6. Agranat, M.B., S.I. Ashitkov, A.B. Granovskii, and G.I. Rukman, Interaction of picosecond laser pulses with the electron, spin, and phonon subsystems of nickel. Sov. Phys. JETP, 1984. 59(4): p. 804-806,

7. Fognini, A., G. Salvatella, R. Gort, T. Michlmayr, A. Vaterlaus, and Y. Acremann, The influence of the excitation pulse length on ultrafast magnetization dynamics in nickel. Structural Dynamics, 2015. 2(2): p. 024501, 10.1063/1.4914891.

8. Koopmans, B., G. Malinowski, F. Dalla Longa, D. Steiauf, M. Fahnle, T. Roth, M. Cinchetti, and M. Aeschlimann, Explaining the paradoxical diversity of ultrafast laser-induced demagnetization. Nat Mater, 2010. 9(3): p. 259-65, 10.1038/nmat2593.

9. Stanciu, C.D., F. Hansteen, A.V. Kimel, A. Kirilyuk, A. Tsukamoto, A. Itoh, and T. Rasing, All-Optical Magnetic Recording with Circularly Polarized Light. Physical Review Letters, 2007. 99(4), 10.1103/PhysRevLett.99.047601. 
10. Ostler, T.A., J. Barker, R.F. Evans, R.W. Chantrell, U. Atxitia, O. ChubykaloFesenko, S. El Moussaoui, L. Le Guyader, E. Mengotti, L.J. Heyderman, F. Nolting, A. Tsukamoto, A. Itoh, D. Afanasiev, B.A. Ivanov, A.M. Kalashnikova, K. Vahaplar, J. Mentink, A. Kirilyuk, T. Rasing, and A.V. Kimel, Ultrafast heating as a sufficient stimulus for magnetization reversal in a ferrimagnet. Nat Commun, 2012. 3: p. 666, 10.1038/ncomms1666.

11. Hashimoto, Y., A.R. Khorsand, M. Savoini, B. Koene, D. Bossini, A. Tsukamoto, A. Itoh, Y. Ohtsuka, K. Aoshima, A.V. Kimel, A. Kirilyuk, and T. Rasing, Ultrafast timeresolved magneto-optical imaging of all-optical switching in GdFeCo with femtosecond time-resolution and a mum spatial-resolution. Rev Sci Instrum, 2014. 85(6): p. 063702, 10.1063/1.4880015.

12. Radu, I., K. Vahaplar, C. Stamm, T. Kachel, N. Pontius, H.A. Durr, T.A. Ostler, J. Barker, R.F. Evans, R.W. Chantrell, A. Tsukamoto, A. Itoh, A. Kirilyuk, T. Rasing, and A.V. Kimel, Transient ferromagnetic-like state mediating ultrafast reversal of antiferromagnetically coupled spins. Nature, 2011. 472(7342): p. 205-8, 10.1038/nature09901.

13. Johnson, M.T., P.J.H. Bloemen, F.J.A.d. Broeder, and J.J.d. Vries, Magnetic anisotropy in metallic multilayers. Reports on Progress in Physics, 1996. 59(11): p. 1409, http://stacks.iop.org/0034-4885/59/i=11/a=002

14. Qiu, Z.Q. and S.D. Bader, Surface magneto-optic Kerr effect. Review of Scientific Instruments, 2000. 71(3): p. 1243, 10.1063/1.1150496.

15. Vahaplar, K., A.M. Kalashnikova, A.V. Kimel, S. Gerlach, D. Hinzke, U. Nowak, R. Chantrell, A. Tsukamoto, A. Itoh, A. Kirilyuk, and T. Rasing, All-optical magnetization reversal by circularly polarized laser pulses: Experiment and multiscale modeling. Physical Review B, 2012. 85(10), 10.1103/PhysRevB.85.104402.

16. Khorsand, A.R., M. Savoini, A. Kirilyuk, A.V. Kimel, A. Tsukamoto, A. Itoh, and T. Rasing, Role of Magnetic Circular Dichroism in All-Optical Magnetic Recording. Physical Review Letters, 2012. 108(12), 10.1103/PhysRevLett.108.127205.

17. Tudosa, I., C. Stamm, A.B. Kashuba, F. King, H.C. Siegmann, J. Stohr, G. Ju, B. Lu, and D. Weller, The ultimate speed of magnetic switching in granular recording media. Nature, 2004. 428(6985): p. 831-833, http://www.nature.com/nature/journal/v428/n6985/suppinfo/nature02438_S1. html.

18. Schubert, C., A. Hassdenteufel, P. Matthes, J. Schmidt, M. Helm, R. Bratschitsch, and $\mathrm{M}$. Albrecht, All-optical helicity dependent magnetic switching in an artificial zero moment magnet. Applied Physics Letters, 2014. 104(8), 10.1063/1.4866803. 
19. Mangin, S., M. Gottwald, C.H. Lambert, D. Steil, V. Uhlir, L. Pang, M. Hehn, S. Alebrand, M. Cinchetti, G. Malinowski, Y. Fainman, M. Aeschlimann, and E.E. Fullerton, Engineered materials for all-optical helicity-dependent magnetic switching. Nature Materials, 2014. 13(3): p. 287-293, 10.1038/nmat3864.

20. Lambert, C.H., S. Mangin, B.S.D.C.S. Varaprasad, Y.K. Takahashi, M. Hehn, M. Cinchetti, G. Malinowski, K. Hono, Y. Fainman, M. Aeschlimann, and E.E. Fullerton, All-optical control of ferromagnetic thin films and nanostructures. Science, 2014. 345(6202): p. 1337-1340, 10.1126/science.1253493.

21. El Hadri, M.S., M. Hehn, P. Pirro, C.-H. Lambert, G. Malinowski, E.E. Fullerton, and S. Mangin, Domain size criterion for the observation of all-optical helicitydependent switching in magnetic thin films. Physical Review B, 2016. 94(6), 10.1103/PhysRevB.94.064419.

22. Medapalli, R., D. Afanasiev, D.K. Kim, Y. Quessab, S. Manna, S.A. Montoya, A. Kirilyuk, T. Rasing, A. Kimel, and E.E. Fullerton, Mechanism of all-optical control of ferromagnetic multilayers with circularly polarized light. arXiv:1607.02505, 2016,

23. Takahashi, Y.K., R. Medapalli, S. Kasai, J. Wang, K. Ishioka, S.H. Wee, O. Hellwig, K. Hono, and E.E. Fullerton, Accumulative Magnetic Switching of UltrahighDensity Recording Media by Circularly Polarized Light. Physical Review Applied, 2016. 6(5), 10.1103/PhysRevApplied.6.054004.

24. Stupakiewicz, A., K. Szerenos, D. Afanasiev, A. Kirilyuk, and A.V. Kimel, Ultrafast nonthermal photo-magnetic recording in a transparent medium. Nature, 2017. 542(7639): p. 71-+, 10.1038/nature20807.

25. Liu, T.-M., T. Wang, A.H. Reid, M. Savoini, X. Wu, B. Koene, P. Granitzka, C.E. Graves, D.J. Higley, Z. Chen, G. Razinskas, M. Hantschmann, A. Scherz, J. Stoehr, A. Tsukarnote, B. Hecht, A.V. Kimel, A. Kirilyuk, T. Rasing, and H.A. Duerr, Nanoscale Confinement of All-Optical Magnetic Switching in TbFeCo Competition with Nanoscale Heterogeneity. Nano Letters, 2015. 15(10): p. 68626868, 10.1021/acs.nanolett.5b02743.

26. Gierster, L., A.A. Uenal, L. Pape, F. Radu, and F. Kronast, Laser induced magnetization switching in a TbFeCo ferrimagnetic thin film: discerning the impact of dipolar fields, laser heating and laser helicity by XPEEM. Ultramicroscopy, 2015. 159: p. 508-512, 10.1016/j.ultramic.2015.05.016.

27. Le Guyader, L., S. El Moussaoui, M. Buzzi, M. Savoini, A. Tsukamoto, A. Itoh, A. Kirilyuk, T. Rasing, F. Nolting, and A.V. Kimel, Deterministic character of alloptical magnetization switching in GdFe-based ferrimagnetic alloys. Physical Review B, 2016. 93(13), 10.1103/PhysRevB.93.134402. 
28. El Hadri, M.S., P. Pirro, C.H. Lambert, N. Bergeard, S. Petit-Watelot, M. Hehn, G. Malinowski, F. Montaigne, Y. Quessab, R. Medapalli, E.E. Fullerton, and S. Mangin, Electrical characterization of all-optical helicity-dependent switching in ferromagnetic Hall crosses. Applied Physics Letters, 2016. 108(9), 10.1063/1.4943107.

29. Finazzi, M., M. Savoini, A.R. Khorsand, A. Tsukamoto, A. Itoh, L. Duo, A. Kirilyuk, T. Rasing, and M. Ezawa, Laser-Induced Magnetic Nanostructures with Tunable Topological Properties. Physical Review Letters, 2013. 110(17), 10.1103/PhysRevLett.110.177205.

30. Montoya, S.A., S. Couture, J.J. Chess, J.C.T. Lee, N. Kent, D. Henze, S.K. Sinha, M.Y. Im, S.D. Kevan, P. Fischer, B.J. McMorran, V. Lomakin, S. Roy, and E.E. Fullerton, Tailoring magnetic energies to form dipole skyrmions and skyrmion lattices. Physical Review B, 2017. 95(2), 10.1103/PhysRevB.95.024415.

31. Atxitia, U., P. Nieves, and O. Chubykalo-Fesenko, Landau-Lifshitz-Bloch equation for ferrimagnetic materials. Physical Review B, 2012. 86(10), 10.1103/PhysRevB.86.104414.

32. Ostler, T.A., J. Barker, R.F.L. Evans, R.W. Chantrell, U. Atxitia, O. ChubykaloFesenko, S. El Moussaoui, L. Le Guyader, E. Mengotti, L.J. Heyderman, F. Nolting, A. Tsukamoto, A. Itoh, D. Afanasiev, B.A. Ivanov, A.M. Kalashnikova, K. Vahaplar, J. Mentink, A. Kirilyuk, T. Rasing, and A.V. Kimel, Ultrafast heating as a sufficient stimulus for magnetization reversal in a ferrimagnet. Nature Communications, 2012. 3, 10.1038/ncomms1666.

33. Oniciuc, E., L. Stoleriu, D. Cimpoesu, and A. Stancu, Effect of damping on the laser induced ultrafast switching in rare earth-transition metal alloys. Applied Physics Letters, 2014. 104(22), 10.1063/1.4881135.

34. Xu, C., T.A. Ostler, and R.W. Chantrell, Thermally induced magnetization switching in Gd/Fe multilayers. Physical Review B, 2016. 93(5), 10.1103/PhysRevB.93.054302.

35. Cornelissen, T.D., R. Cordoba, and B. Koopmans, Microscopic model for all optical switching in ferromagnets. Applied Physics Letters, 2016. 108(14), 10.1063/1.4945660.

36. Nieves, P. and O. Chubykalo-Fesenko, Modeling of Ultrafast Heat-and FieldAssisted Magnetization Dynamics in FePt. Physical Review Applied, 2016. 5(1), 10.1103/PhysRevApplied.5.014006.

37. Berritta, M., R. Mondal, K. Carva, and P.M. Oppeneer, Ab Initio Theory of Coherent Laser-Induced Magnetization in Metals. Physical Review Letters, 2016. 117(13), 10.1103/PhysRevLett.117.137203. 
38. Ellis, M.O.A., E.E. Fullerton, and R.W. Chantrell, All-optical switching in granular ferromagnets caused by magnetic circular dichroism. Scientific Reports, 2016. 6, 10.1038/srep30522.

39. Gorchon, J., Y. Yang, and J. Bokor, Model for multishot all-thermal all-optical switching in ferromagnets. Physical Review B, 2016. 94(2),

10.1103/PhysRevB.94.020409.

40. Staerk, M., F. Schlickeiser, D. Nissen, B. Hebler, P. Graus, D. Hinzke, E. Scheer, P. Leiderer, M. Fonin, M. Albrecht, U. Nowak, and J. Boneberg, Controlling the magnetic structure of $\mathrm{Co} / \mathrm{Pd}$ thin films by direct laser interference patterning. Nanotechnology, 2015. 26(20), 10.1088/0957-4484/26/20/205302.

41. El Hadri, M.S., P. Pirro, C.H. Lambert, S. Petit-Watelot, Y. Quessab, M. Hehn, F. Montaigne, G. Malinowski, and S. Mangin, Two types of all-optical magnetization switching mechanisms using femtosecond laser pulses. Physical Review B, 2016. 94(6), 10.1103/PhysRevB.94.064412.

42. Sudrie, L., M. Franco, B. Prade, and A. Mysyrowicz, Study of damage in fused silica induced by ultra-short IR laser pulses. Optics Communications, 2001. 191(36): p. 333-339, 10.1016/s0030-4018(01)01152-x.

43. Yang, P., G.R. Burns, J.P. Guo, T.S. Luk, and G.A. Vawter, Femtosecond laserpulse-induced birefringence in optically isotropic glass. Journal of Applied Physics, 2004. 95(10): p. 5280-5283, 10.1063/1.1707231.

44. Bhardwaj, V.R., E. Simova, P.P. Rajeev, C. Hnatovsky, R.S. Taylor, D.M. Rayner, and P.B. Corkum, Optically Produced Arrays of Planar Nanostructures inside Fused Silica. Physical Review Letters, 2006. 96(5): p. 057404, https://link.aps.org/doi/10.1103/PhysRevLett.96.057404

45. Cheng, G., K. Mishchik, C. Mauclair, E. Audouard, and R. Stoian, Ultrafast laser photoinscription of polarization sensitive devices in bulk silica glass. Optics Express, 2009. 17(12): p. 9515-9525, 10.1364/oe.17.009515.

46. Koopmans, B., J.J.M. Ruigrok, F.D. Longa, and W.J.M. de Jonge, Unifying ultrafast magnetization dynamics. Physical Review Letters, 2005. 95(26),

10.1103/PhysRevLett.95.267207.

47. Guenther, S., C. Spezzani, R. Ciprian, C. Grazioli, B. Ressel, M. Coreno, L. Poletto, P. Miotti, M. Sacchi, G. Panaccione, V. Uhlir, E.E. Fullerton, G. De Ninno, and C.H. Back, Testing spin-flip scattering as a possible mechanism of ultrafast demagnetization in ordered magnetic alloys. Physical Review B, 2014. 90(18), 10.1103/PhysRevB.90.180407.

48. Kuiper, K.C., T. Roth, A.J. Schellekens, O. Schmitt, B. Koopmans, M. Cinchetti, and M. Aeschlimann, Spin-orbit enhanced demagnetization rate in Co/Pt-multilayers. Applied Physics Letters, 2014. 105(20), 10.1063/1.4902069. 
49. Schmidt, A.J., X. Chen, and G. Chen, Pulse accumulation, radial heat conduction, and anisotropic thermal conductivity in pump-probe transient thermoreflectance. Review of Scientific Instruments, 2008. 79(11), 10.1063/1.3006335.

50. Jaeger, J.C., Pulsed surface heating of a semi-infinite solid. Quarterly of Applied Mathematics, 1953. 11: p. 132-137, 10.1090/qam/99981.

51. Thiele, A.A., The theory of cylindrical magnetic domains. The Bell System Technical Journal, 1969. 48(10): p. 3287-3335, 10.1002/j.15387305.1969.tb01747.x.

52. Thiele, A.A., A.H. Bobeck, Dellator.E, and U.F. Gianola, ENERGY AND GENERAL TRANSLATION FORCE OF CYLINDRICAL MAGNETIC DOMAINS. Bell System Technical Journal, 1971. 50(3): p. 711-+, <Go to ISI>://WOS:A1971J092300001

53. Thiele, A.A., DEVICE IMPLICATIONS OF THEORY OF CYLINDRICAL MAGNETIC DOMAINS. Bell System Technical Journal, 1971. 50(3): p. 725-+, <Go to ISI>://WOS:A1971J092300002

54. Ashkin, A. and J.M. Dziedzic, INTERACTION OF LASER LIGHT WITH MAGNETIC DOMAINS. Applied Physics Letters, 1972. 21(6): p. 253-\&, 10.1063/1.1654366.

55. Kaneko, M., T. Okamoto, H. Tamada, and T. Yamada, OPTICAL OPERATION OF A MAGNETIC-BUBBLE. leee Transactions on Magnetics, 1986. 22(1): p. 2-10, 10.1109/tmag.1986.1064268.

56. Callen, H. and R.M. Josephs, DYNAMICS OF MAGNETIC BUBBLE DOMAINS WITH AN APPLICATION TO WALL MOBILITIES. Journal of Applied Physics, 1971. 42(5): p. 1977-\&, 10.1063/1.1660475.

57. Rossol, F., Temperature dependence of rare-earth orthoferrite properties relevant to propagating domain device applications. IEEE Transactions on Magnetics, 1969. 5(3): p. 562-565, 10.1109/TMAG.1969.1066518.

58. Schryer, N.L. and L.R. Walker, MOTION OF 180 DEGREES DOMAIN-WALLS IN UNIFORM DC MAGNETIC-FIELDS. Journal of Applied Physics, 1974. 45(12): p. 5406-5421, 10.1063/1.1663252.

59. Hassdenteufel, A., J. Schmidt, C. Schubert, B. Hebler, M. Helm, M. Albrecht, and R. Bratschitsch, Low-remanence criterion for helicity-dependent all-optical magnetic switching in ferrimagnets. Physical Review B, 2015. 91(10), 10.1103/PhysRevB.91.104431.

60. Hashimoto, Y., A.R. Khorsand, M. Savoini, B. Koene, D. Bossini, A. Tsukamoto, A. Itoh, Y. Ohtsuka, K. Aoshima, A.V. Kimel, A. Kirilyuk, and T. Rasing, Ultrafast timeresolved magneto-optical imaging of all-optical switching in GdFeCo with femtosecond time-resolution and a mu $m$ spatial-resolution. Review of Scientific Instruments, 2014. 85(6), 10.1063/1.4880015. 
61. Tsema, Y., G. Kichin, O. Hellwig, V. Mehta, A.V. Kimel, A. Kirilyuk, and T. Rasing, Helicity and field dependent magnetization dynamics of ferromagnetic Co/Pt multilayers. Applied Physics Letters, 2016. 109(7), 10.1063/1.4961246.

62. Koopmans, B., J.J.M. Ruigrok, F.D. Longa, and W.J.M. de Jonge, Unifying Ultrafast Magnetization Dynamics. Physical Review Letters, 2005. 95(26): p. 267207, https://link.aps.org/doi/10.1103/PhysRevLett.95.267207

63. Koopmans, B., G. Malinowski, F. Dalla Longa, D. Steiauf, M. Fähnle, T. Roth, M. Cinchetti, and M. Aeschlimann, Explaining the paradoxical diversity of ultrafast laser-induced demagnetization. Nature Materials, 2009. 9: p. 259, $10.1038 /$ nmat2593 https://www.nature.com/articles/nmat2593\#supplementary-information.

64. Günther, S., C. Spezzani, R. Ciprian, C. Grazioli, B. Ressel, M. Coreno, L. Poletto, P. Miotti, M. Sacchi, G. Panaccione, V. Uhlír, E.E. Fullerton, G. De Ninno, and C.H. Back, Testing spin-flip scattering as a possible mechanism of ultrafast demagnetization in ordered magnetic alloys. Physical Review B, 2014. 90(18): p. 180407, https://link.aps.org/doi/10.1103/PhysRevB.90.180407

65. Kuiper, K.C., T. Roth, A.J. Schellekens, O. Schmitt, B. Koopmans, M. Cinchetti, and M. Aeschlimann, Spin-orbit enhanced demagnetization rate in Co/Pt-multilayers. Applied Physics Letters, 2014. 105(20): p. 202402, 10.1063/1.4902069.

66. Mendil, J., P. Nieves, O. Chubykalo-Fesenko, J. Walowski, T. Santos, S. Pisana, and M. Münzenberg, Resolving the role of femtosecond heated electrons in ultrafast spin dynamics. Scientific Reports, 2014. 4: p. 3980, 10.1038/srep03980 https://www.nature.com/articles/srep03980\#supplementary-information.

67. Tsema, Y., G. Kichin, O. Hellwig, V. Mehta, A.V. Kimel, A. Kirilyuk, and T. Rasing, Helicity and field dependent magnetization dynamics of ferromagnetic Co/Pt multilayers. Applied Physics Letters, 2016. 109(7): p. 072405, 10.1063/1.4961246.

68. Roth, T., A.J. Schellekens, S. Alebrand, O. Schmitt, D. Steil, B. Koopmans, M. Cinchetti, and M. Aeschlimann, Temperature Dependence of Laser-Induced Demagnetization in Ni: A Key for Identifying the Underlying Mechanism. Physical Review X, 2012. 2(2): p. 021006, https://link.aps.org/doi/10.1103/PhysRevX.2.021006

69. Ute, B., T.W. Sebastian, S. David, B. Moritz, M. Anna-Katharina, M. Stefan, S. Hans Christian, S. Benjamin, A. Martin, and R. Baerbel, Ultrafast magnetization dynamics in Nickel: impact of pump photon energy. Journal of Physics: Condensed Matter, 2017. 29(24): p. 244002, http://stacks.iop.org/0953$8984 / 29 / i=24 / a=244002$ 
70. Kamil, B., G. Markus, P. Niko, S.-L. Christian, T. Christoph, W. Marko, F. Björn, and $W$. Martin, Influence of the pump pulse wavelength on the ultrafast demagnetization of $\mathrm{Gd}(0001)$ thin films. Journal of Physics: Condensed Matter, 2017. 29(23): p. 234003, http://stacks.iop.org/0953-8984/29/i=23/a=234003

71. Kazantseva, N., U. Nowak, R.W. Chantrell, J. Hohlfeld, and A. Rebei, Slow recovery of the magnetisation after a sub-picosecond heat pulse. EPL (Europhysics Letters), 2008. 81(2): p. 27004, http://stacks.iop.org/0295$5075 / 81 / i=2 / a=27004$

72. Hinzke, D., U. Atxitia, K. Carva, P. Nieves, O. Chubykalo-Fesenko, P.M. Oppeneer, and U. Nowak, Multiscale modeling of ultrafast element-specific magnetization dynamics of ferromagnetic alloys. Physical Review B, 2015. 92(5): p. 054412, https://link.aps.org/doi/10.1103/PhysRevB.92.054412

73. Battiato, M., K. Carva, and P.M. Oppeneer, Superdiffusive Spin Transport as a Mechanism of Ultrafast Demagnetization. Physical Review Letters, 2010. 105(2): p. 027203, https://link.aps.org/doi/10.1103/PhysRevLett.105.027203

74. Schellekens, A.J., W. Verhoeven, T.N. Vader, and B. Koopmans, Investigating the contribution of superdiffusive transport to ultrafast demagnetization of ferromagnetic thin films. Applied Physics Letters, 2013. 102(25): p. 252408, 10.1063/1.4812658.

75. von Korff Schmising, C., B. Pfau, M. Schneider, C.M. Günther, M. Giovannella, J. Perron, B. Vodungbo, L. Müller, F. Capotondi, E. Pedersoli, N. Mahne, J. Lüning, and S. Eisebitt, Imaging Ultrafast Demagnetization Dynamics after a Spatially Localized Optical Excitation. Physical Review Letters, 2014. 112(21): p. 217203, https://link.aps.org/doi/10.1103/PhysRevLett.112.217203

76. Schmising, C.v.K., M. Giovannella, D. Weder, S. Schaffert, J.L. Webb, and S. Eisebitt, Nonlocal ultrafast demagnetization dynamics of Co/Pt multilayers by optical field enhancement. New Journal of Physics, 2015. 17(3): p. 033047, http://stacks.iop.org/1367-2630/17/i=3/a=033047

77. Eschenlohr, A., L. Persichetti, T. Kachel, M. Gabureac, P. Gambardella, and C. Stamm, Spin currents during ultrafast demagnetization of ferromagnetic bilayers. Journal of Physics: Condensed Matter, 2017. 29(38): p. 384002, http://stacks.iop.org/0953-8984/29/i=38/a=384002

78. Eschenlohr, A., M. Battiato, P. Maldonado, N. Pontius, T. Kachel, K. Holldack, R. Mitzner, A. Föhlisch, P.M. Oppeneer, and C. Stamm, Ultrafast spin transport as key to femtosecond demagnetization. Nature Materials, 2013. 12: p. 332, $10.1038 /$ nmat3546. 
79. Salvatella, G., R. Gort, K. Bühlmann, S. Däster, A. Vaterlaus, and Y. Acremann, Ultrafast demagnetization by hot electrons: Diffusion or super-diffusion?

Structural Dynamics, 2016. 3(5): p. 055101, 10.1063/1.4964892.

80. Vodungbo, B., B. Tudu, J. Perron, R. Delaunay, L. Müller, M.H. Berntsen, G.

Grübel, G. Malinowski, C. Weier, J. Gautier, G. Lambert, P. Zeitoun, C. Gutt, E. Jal, A.H. Reid, P.W. Granitzka, N. Jaouen, G.L. Dakovski, S. Moeller, M.P. Minitti, A.

Mitra, S. Carron, B. Pfau, C. von Korff Schmising, M. Schneider, S. Eisebitt, and J.

Lüning, Indirect excitation of ultrafast demagnetization. Scientific Reports, 2016.

6: p. 18970, 10.1038/srep18970.

81. Bergeard, N., M. Hehn, S. Mangin, G. Lengaigne, F. Montaigne, M.L.M. Lalieu, B. Koopmans, and G. Malinowski, Hot-Electron-Induced Ultrafast Demagnetization in \$ $\mid$ mathrm\{Co\}/Amathrm\{Pt\}\$ Multilayers. Physical Review Letters, 2016. 117(14): p. 147203, https://link.aps.org/doi/10.1103/PhysRevLett.117.147203

82. Stanciu, C.D., F. Hansteen, A.V. Kimel, A. Kirilyuk, A. Tsukamoto, A. Itoh, and T. Rasing, All-Optical Magnetic Recording with Circularly Polarized Light. Physical Review Letters, 2007. 99(4): p. 047601, https://link.aps.org/doi/10.1103/PhysRevLett.99.047601

83. Mangin, S., M. Gottwald, C.H. Lambert, D. Steil, V. Uhlír, L. Pang, M. Hehn, S. Alebrand, M. Cinchetti, G. Malinowski, Y. Fainman, M. Aeschlimann, and E.E. Fullerton, Engineered materials for all-optical helicity-dependent magnetic switching. Nature Materials, 2014. 13: p. 286, 10.1038/nmat3864 https://www.nature.com/articles/nmat3864\#supplementary-information.

84. Lambert, C.-H., S. Mangin, B.S.D.C.S. Varaprasad, Y.K. Takahashi, M. Hehn, M. Cinchetti, G. Malinowski, K. Hono, Y. Fainman, M. Aeschlimann, and E.E. Fullerton, All-optical control of ferromagnetic thin films and nanostructures. Science, 2014. 345(6202): p. 1337-1340, 10.1126/science.1253493.

85. El Hadri, M.S., M. Hehn, P. Pirro, C.-H. Lambert, G. Malinowski, E.E. Fullerton, and S. Mangin, Domain size criterion for the observation of all-optical helicitydependent switching in magnetic thin films. Physical Review B, 2016. 94(6): p. 064419, https://link.aps.org/doi/10.1103/PhysRevB.94.064419

86. El Hadri, M.S., P. Pirro, C.H. Lambert, S. Petit-Watelot, Y. Quessab, M. Hehn, F. Montaigne, G. Malinowski, and S. Mangin, Two types of all-optical magnetization switching mechanisms using femtosecond laser pulses. Physical Review B, 2016. 94(6): p. 064412, https://link.aps.org/doi/10.1103/PhysRevB.94.064412

87. El Hadri, M.S., P. Pirro, C.H. Lambert, N. Bergeard, S. Petit-Watelot, M. Hehn, G. Malinowski, F. Montaigne, Y. Quessab, R. Medapalli, E.E. Fullerton, and S. Mangin, Electrical characterization of all-optical helicity-dependent switching in 
ferromagnetic Hall crosses. Applied Physics Letters, 2016. 108(9): p. 092405, 10.1063/1.4943107.

88. Hoveyda, F., E. Hohenstein, and S. Smadici, Heat accumulation and all-optical switching by domain wall motion in Co/Pd superlattices. Journal of Physics: Condensed Matter, 2017. 29(22): p. 225801, http://stacks.iop.org/0953$8984 / 29 / \mathrm{i}=22 / \mathrm{a}=225801$

89. Takahashi, Y.K., R. Medapalli, S. Kasai, J. Wang, K. Ishioka, S.H. Wee, O. Hellwig, K. Hono, and E.E. Fullerton, Accumulative Magnetic Switching of UltrahighDensity Recording Media by Circularly Polarized Light. Physical Review Applied, 2016. 6(5): p. 054004, https://link.aps.org/doi/10.1103/PhysRevApplied.6.054004

90. John, R., M. Berritta, D. Hinzke, C. Müller, T. Santos, H. Ulrichs, P. Nieves, J. Walowski, R. Mondal, O. Chubykalo-Fesenko, J. McCord, P.M. Oppeneer, U. Nowak, and M. Münzenberg, Magnetisation switching of FePt nanoparticle recording medium by femtosecond laser pulses. Scientific Reports, 2017. 7(1): p. 4114, 10.1038/s41598-017-04167-w.

91. Hashimoto, Y., A.R. Khorsand, M. Savoini, B. Koene, D. Bossini, A. Tsukamoto, A. Itoh, Y. Ohtsuka, K. Aoshima, A.V. Kimel, A. Kirilyuk, and T. Rasing, Ultrafast timeresolved magneto-optical imaging of all-optical switching in GdFeCo with femtosecond time-resolution and a $\mu \mathrm{m}$ spatial-resolution. Review of Scientific Instruments, 2014. 85(6): p. 063702, 10.1063/1.4880015.

92. Cornelissen, T.D., R. Córdoba, and B. Koopmans, Microscopic model for all optical switching in ferromagnets. Applied Physics Letters, 2016. 108(14): p. 142405, 10.1063/1.4945660.

93. Nieves, P. and O. Chubykalo-Fesenko, Modeling of Ultrafast Heat- and FieldAssisted Magnetization Dynamics in FePt. Physical Review Applied, 2016. 5(1): p. 014006, https://link.aps.org/doi/10.1103/PhysRevApplied.5.014006

94. Berritta, M., R. Mondal, K. Carva, and P.M. Oppeneer, Ab Initio. Physical Review Letters, 2016. 117(13): p. 137203, https://link.aps.org/doi/10.1103/PhysRevLett.117.137203

95. Kirilyuk, A., A.V. Kimel, and T. Rasing, Ultrafast optical manipulation of magnetic order. Reviews of Modern Physics, 2010. 82(3): p. 2731-2784, https://link.aps.org/doi/10.1103/RevModPhys.82.2731

96. Ostler, T.A., J. Barker, R.F.L. Evans, R.W. Chantrell, U. Atxitia, O. ChubykaloFesenko, S. El Moussaoui, L. Le Guyader, E. Mengotti, L.J. Heyderman, F. Nolting, A. Tsukamoto, A. Itoh, D. Afanasiev, B.A. Ivanov, A.M. Kalashnikova, K. Vahaplar, J. Mentink, A. Kirilyuk, T. Rasing, and A.V. Kimel, Ultrafast heating as a sufficient 
stimulus for magnetization reversal in a ferrimagnet. Nature Communications, 2012. 3: p. 666, 10.1038/ncomms1666.

97. Schellekens, A.J. and B. Koopmans, Microscopic model for ultrafast magnetization dynamics of multisublattice magnets. Physical Review B, 2013. 87(2): p. 020407, https://link.aps.org/doi/10.1103/PhysRevB.87.020407

98. Oniciuc, E., L. Stoleriu, D. Cimpoesu, and A. Stancu, Effect of damping on the laser induced ultrafast switching in rare earth-transition metal alloys. Applied Physics Letters, 2014. 104(22): p. 222404, 10.1063/1.4881135.

99. Atxitia, U., T.A. Ostler, R.W. Chantrell, and O. Chubykalo-Fesenko, Optimal electron, phonon, and magnetic characteristics for low energy thermally induced magnetization switching. Applied Physics Letters, 2015. 107(19): p. 192402, 10.1063/1.4935416.

100. Gorchon, J., Y. Yang, and J. Bokor, Model for multishot all-thermal all-optical switching in ferromagnets. Physical Review B, 2016. 94(2): p. 020409, https://link.aps.org/doi/10.1103/PhysRevB.94.020409

101. Khorsand, A.R., M. Savoini, A. Kirilyuk, A.V. Kimel, A. Tsukamoto, A. Itoh, and T. Rasing, Role of Magnetic Circular Dichroism in All-Optical Magnetic Recording. Physical Review Letters, 2012. 108(12): p. 127205, https://link.aps.org/doi/10.1103/PhysRevLett.108.127205

102. Ellis, M.O.A., E.E. Fullerton, and R.W. Chantrell, All-optical switching in granular ferromagnets caused by magnetic circular dichroism. Scientific Reports, 2016. 6: p. 30522, 10.1038/srep30522.

103. Liu, Z., R. Brandt, O. Hellwig, S. Florez, T. Thomson, B. Terris, and H. Schmidt, Thickness dependent magnetization dynamics of perpendicular anisotropy Co/Pd multilayer films. Journal of Magnetism and Magnetic Materials, 2011. 323(12): p. 1623-1626, https://doi.org/10.1016/j.jmmm.2011.01.025.

104. Pal, S., B. Rana, O. Hellwig, T. Thomson, and A. Barman, Tunable magnonic frequency and damping in [Co/Pd]8 multilayers with variable Co layer thickness. Applied Physics Letters, 2011. 98(8): p. 082501, 10.1063/1.3559222.

105. Bigot, J.Y., M. Vomir, L.H.F. Andrade, and E. Beaurepaire, Ultrafast magnetization dynamics in ferromagnetic cobalt: The role of the anisotropy. Chemical Physics, 2005. 318(1): p. 137-146, https://doi.org/10.1016/j.chemphys.2005.06.016.

106. Hohlfeld, J., S.S. Wellershoff, J. Güdde, U. Conrad, V. Jähnke, and E. Matthias, Electron and lattice dynamics following optical excitation of metals. Chemical Physics, 2000. 251(1): p. 237-258, https://doi.org/10.1016/S03010104(99)00330-4.

107. Siegel, J., D. Puerto, W. Gawelda, G. Bachelier, J. Solis, L. Ehrentraut, and J. Bonse, Plasma formation and structural modification below the visible ablation 
threshold in fused silica upon femtosecond laser irradiation. Applied Physics Letters, 2007. 91(8): p. 082902, 10.1063/1.2766848.

108. Hoveyda, F., M. Adnani, and S. Smadici, Heat diffusion in magnetic superlattices on glass substrates. Journal of Applied Physics, 2017. 122(18): p. 184304, 10.1063/1.5002669.

109. Boeglin, C., E. Beaurepaire, V. Halté, V. López-Flores, C. Stamm, N. Pontius, H.A. Dürr, and J.Y. Bigot, Distinguishing the ultrafast dynamics of spin and orbital moments in solids. Nature, 2010. 465: p. 458, 10.1038/nature09070 https://www.nature.com/articles/nature09070\#supplementary-information.

110. Back, C.H., R. Allenspach, W. Weber, S.S.P. Parkin, D. Weller, E.L. Garwin, and H.C. Siegmann, Minimum Field Strength in Precessional Magnetization Reversal. Science, 1999. 285(5429): p. 864-867, 10.1126/science.285.5429.864.

111. Bauer, M., R. Lopusnik, J. Fassbender, and B. Hillebrands, Magnetization reversal in ultrashort magnetic field pulses. Journal of Magnetism and Magnetic Materials, 2000. 218(2): p. 165-176, https://doi.org/10.1016/S03048853(00)00400-5.

112. Shieh, H.P.D. and M.H. Kryder, Dynamics and factors controlling regularity of thermomagnetically written domains. Journal of Applied Physics, 1987. 61(3): p. 1108-1122, 10.1063/1.338153.

113. Ogasawara, T., N. Iwata, Y. Murakami, H. Okamoto, and Y. Tokura, Submicronscale spatial feature of ultrafast photoinduced magnetization reversal in TbFeCo thin film. Applied Physics Letters, 2009. 94(16): p. 162507, 10.1063/1.3123256.

114. Gierster, L., A.A. Ünal, L. Pape, F. Radu, and F. Kronast, Laser induced magnetization switching in a TbFeCo ferrimagnetic thin film: discerning the impact of dipolar fields, laser heating and laser helicity by XPEEM.

Ultramicroscopy, 2015. 159(Part 3): p. 508-512, https://doi.org/10.1016/j.ultramic.2015.05.016.

115. Le Guyader, L., M. Savoini, S. El Moussaoui, M. Buzzi, A. Tsukamoto, A. Itoh, A. Kirilyuk, T. Rasing, A.V. Kimel, and F. Nolting, Nanoscale sub-100 picosecond alloptical magnetization switching in GdFeCo microstructures. Nature Communications, 2015. 6: p. 5839, 10.1038/ncomms6839.

116. Le Guyader, L., S. El Moussaoui, M. Buzzi, M. Savoini, A. Tsukamoto, A. Itoh, A. Kirilyuk, T. Rasing, F. Nolting, and A.V. Kimel, Deterministic character of alloptical magnetization switching in GdFe-based ferrimagnetic alloys. Physical Review B, 2016. 93(13): p. 134402, https://link.aps.org/doi/10.1103/PhysRevB.93.134402

117. The Object Oriented MicroMagnetic Framework (OOMMF) project at ITL/NIST. 2006. 
118. Moreno, R., R.F.L. Evans, S. Khmelevskyi, M.C. Muñoz, R.W. Chantrell, and O. Chubykalo-Fesenko, Temperature-dependent exchange stiffness and domain wall width in Co. Physical Review B, 2016. 94(10): p. 104433, https://link.aps.org/doi/10.1103/PhysRevB.94.104433

119. Thiele, A.A., A.H. Bobeck, E.D. Torre, and U.F. Gianola, The energy and general translation force of cylindrical magnetic domains. The Bell System Technical Journal, 1971. 50(3): p. 711-724, 10.1002/j.1538-7305.1971.tb01880.x.

120. Bobeck, A.H., Properties and Device Applications of Magnetic Domains in Orthoferrites. Bell System Technical Journal, 1967. 46(8): p. 1901-1925, 10.1002/j.1538-7305.1967.tb03177.x.

121. Gorchon, J., R.B. Wilson, Y. Yang, A. Pattabi, J.Y. Chen, L. He, J.P. Wang, M. Li, and J. Bokor, Role of electron and phonon temperatures in the helicity-independent all-optical switching of GdFeCo. Physical Review B, 2016. 94(18): p. 184406, https://link.aps.org/doi/10.1103/PhysRevB.94.184406

122. Callen, H. and R.M. Josephs, Dynamics of Magnetic Bubble Domains with an Application to Wall Mobilities. Journal of Applied Physics, 1971. 42(5): p. 19771982, 10.1063/1.1660475.

123. Medapalli, R., D. Afanasiev, D. Kim, Y. Quessab, S.A. Monotoya, A. Kirilyuk, T. Rasing, A.V. Kimel, and E.E. Fullerton, Mechanism of all-optical control of ferromagnetic multilayers with circularly polarized light. arXiv:1607.02505v1, 2016 ,

124. Martin, S., S. Frank, N. Dennis, H. Birgit, G. Philipp, H. Denise, S. Elke, L. Paul, F. Mikhail, A. Manfred, N. Ulrich, and B. Johannes, Controlling the magnetic structure of $\mathrm{Co} / \mathrm{Pd}$ thin films by direct laser interference patterning. Nanotechnology, 2015. 26(20): p. 205302, http://stacks.iop.org/0957$4484 / 26 / i=20 / a=205302$

125. Fretigny, C., J.P. Roger, V. Reita, and D. Fournier, Analytical inversion of photothermal measurements: Independent determination of the thermal conductivity and diffusivity of a conductive layer deposited on an insulating substrate. J. Appl. Phys., 2007. 102: p. 116104,

126. Reichling, M. and H. Gronbeck, Harmonic heat flow in isotropic layered systems and its use for thin film thermal conductivity measurements. J. Appl. Phys., 1994. 75(4): p. 1914,

127. Cahill, D.G., P.V. Braun, G. Chen, D.R. Clarke, S. Fan, K.E. Goodson, P. Keblinski, W.P. King, G.D. Mahan, A. Majumdar, H.J. Maris, S.R. Phillpot, E. Pop, and E.L. Shi, Nanoscale thermal transport. II. 2003-2012. Appl. Phys. Rev., 2014. 1: p. 011305, 
128. Schmidt, A., M. Chiesa, X. Chen, and G. Chen, An optical pump-probe technique for measuring the thermal conductivity of liquids. Rev. Sci. Instrum., 2008. 79: p. 064902,

129. Schmidt, A., X. Chen, and G. Chen, Pulse accumulation, radial heat conduction, and anisotropic thermal conductivity in pump-probe transient thermoreflectance. Rev. Sci. Instrum., 2008. 79: p. 114902,

130. de Jong, J.A., A.M. Kalashnikova, R.V. Pisarev, A.M. Balbashov, A.V. Kimel, A. Kirilyuk, and T. Rasing, Effect of laser pulse propagation on ultrafast magnetization dynamics in a birefringent medium. J. Phys.: Condens. Matter, 2017. 29: p. 164004,

131. Afanasiev, D., B.A. Ivanov, R.V. Pisarev, A. Kirilyuk, T. Rasing, and A.V. Kimel, Femtosecond single-shot imaging and control of a laser-induced first-order phase transition in HoFeO3. J. Phys.: Condens. Matter, 2017. 29: p. 224003,

132. Stanciu, C.D., F. Hansteen, A.V. Kimel, A. Kirilyuk, A. Tsukamoto, A. Itoh, and T. Rasing, All-optical magnetic recording with circularly polarized light. Phys. Rev. Lett., 2007. 99: p. 047601,

133. Aktag, A., S. Michalski, L. Yue, R.D. Kirby, and S.H. Liou, Formation of an anisotropy lattice in Co/Pt multilayers by direct laser interference patterning. J. Appl. Phys., 2006. 99: p. 093901,

134. Schuppler, C., A. Habenicht, I.L. Guhr, M. Maret, P. Leiderer, J. Boneberg, and M. Albrecht, Control of magnetic anisotropy and magnetic patterning of perpendicular Co/Pt multilayers by laser irradiation. Appl. Phys. Lett., 2006. 88: p. 012506,

135. Leufke, P.M., S. Riedel, M.S. Lee, J. Li, H. Rohrmann, T. Eimuller, P. Leiderer, J. Boneberg, G. Schatz, and M. Albrecht, Two different coercivity lattices in Co/Pd multilayers generated by single-pulse direct laser interference lithography. J. Appl. Phys., 2009. 105: p. 113915,

136. Kisielewski, J., W. Dobrogowski, Z. Kurant, A. Stupakiewicz, M. Tekielak, A. Kirilyuk, A. Kimel, T. Rasing, L.T. Baczewski, A. Wawro, K. Balin, J. Szade, and A. Maziewski, Irreversible modification of magnetic properties of Pt/Co/Pt ultrathin films by femtosecond laser pulses. J. Appl. Phys., 2014. 115: p. 053906,

137. Stark, M., F. Schlickeiser, D. Nissen, B. Hebler, P. Graus, D. Hinzke, E. Scheer, P. Leiderer, M. Fonin, M. Albrecht, U. Nowak, and J. Boneberg, Controlling the magnetic structure of $\mathrm{Co} / \mathrm{Pd}$ thin films by direct laser interference patterning. Nanotechnology, 2015. 26: p. 205302,

138. Lambert, C.H., S. Mangin, B. Varaprasad, Y.K. Takahashi, M. Hehn, M. Cinchetti, G. Malinowski, K. Hono, Y. Fainman, M. Aeschlimann, and E.E. Fullerton, All- 
optical control of ferromagnetic thin films and nanostructures. Science, 2014. 345: p. 1337,

139. Mangin, S., M. Gottwald, C.H. Lambert, D. Steil, V. Uhlr, L. Pang, M. Hehn, S. Alebrand, M. Cinchetti, G. Malinowski, Y. Fainman, M. Aeschlimann, and E.E. Fullerton, Engineered materials for all-optical helicity-dependent magnetic switching. Nat. Mater., 2014. 13: p. 286,

140. Gorchon, J., C.H. Lambert, Y. Yang, A. Pattabi, R.B. Wilson, S. Salahuddin, and J. Bokor, Single shot ultrafast all optical magnetization switching of ferromagnetic Co/Pt multilayers. Appl. Phys. Lett., 2017. 111: p. 042401,

141. Takahashi, Y.K., R. Medapalli, S. Kasai, J. Wang, K. Ishioka, S.H. Wee, O. Hellwig, K. Hono, and E.E. Fullerton, Accumulative magnetic switching of ultrahighdensity recording media by circularly polarized light. Phys. Rev. Appl., 2016. 6: p. 054004,

142. John, R., M. Berritta, D. Hinzke, C. Muller, T. Santos, H. Ulrichs, P. Nieves, J. Walowski, R. Mondal, O. Chubykalo-Fesenko, J. McCord, P.M. Oppeneer, U. Nowak, and M. Munzenberg, Magnetisation switching of FePt nanoparticle recording medium by femtosecond laser pulses. Sci. Rep., 2017. 7: p. 4114,

143. Cornelissen, T.D., R. Cordoba, and B. Koopmans, Microscopic model for all optical switching in ferromagnets. Appl. Phys. Lett., 2016. 108: p. 142405,

144. Khorsand, A.R., M. Savoini, A. Kirilyuk, A.V. Kimel, A. Tsukamoto, A. Itoh, and T. Rasing, Role of magnetic circular dichroism in all-optical magnetic recording. Phys. Rev. Lett., 2012. 108: p. 127205,

145. Ostler, T.A., J. Barker, R.F.L. Evans, R.W. Chantrell, U. Atxitia, O. ChubykaloFesenko, S. El Moussaoui, L. Le Guyader, E. Mengotti, L.J. Heyderman, F. Nolting, A. Tsukamoto, A. Itoh, D. Afanasiev, B.A. Ivanov, A.M. Kalashnikova, K. Vahaplar, J. Mentink, A. Kirilyuk, T. Rasing, and A.V. Kimel, Ultrafast heating as a sufficient stimulus for magnetization reversal in a ferrimagnet. Nat. Comm., 2012. 3: p. 666 ,

146. Xu, C., T.A. Ostler, and R.W. Chantrell, Thermally induced magnetization switching in Gd/Fe multilayers. Phys. Rev. B, 2016. 93: p. 054302,

147. Ellis, M.O.A., E.E. Fullerton, and R.W. Chantrell, All-optical switching in granular ferromagnets caused by magnetic circular dichroism. Sci. Rep., 2016. 6: p. 30522,

148. Gorchon, J., Y. Yang, and J. Bokor, Model for multishot all-thermal all-optical switching in ferromagnets. Phys. Rev. B, 2016. 94: p. 020409(R),

149. El Hadri, M.S., P. Pirro, C.H. Lambert, S. Petit-Watelot, Y. Quessab, M. Hehn, F. Montaigne, G. Malinowski, and S. Mangin, Two types of all-optical magnetization switching mechanisms using femtosecond laser pulses. Phys Rev B, 2016. 94: p. 064412, 
150. Hoveyda, F., E. Hohenstein, and S. Smadici, Heat accumulation and all-optical switching by domain wall motion in Co/Pd superlattices. J. Phys.: Condens. Matter, 2017. 29: p. 225801,

151. Cahill, D.G., Analysis of heat flow in layered structures for time-domain thermoreflectance. Rev. Sci. Instrum., 2004. 75(12): p. 5119,

152. Schmidt, A.J., R. Cheaito, and M. Chiesa, A frequency-domain thermoreflectance method for the characterization of thermal properties. Rev. Sci. Instrum., 2009. 80: p. 094901,

153. Liu, J., G.M. Choi, and D.G. Cahill, Measurement of the anisotropic thermal conductivity of molybdenum disulfide by the time-resolved magneto-optic Kerr effect. J. Appl. Phys., 2014. 116: p. 233107,

154. Qiu, Z.Q. and S.D. Bader, Surface magneto-optic Kerr effect. Rev. Sci. Instr., 2000. 71(3): p. 1243,

155. Hoveyda, F., E. Hohenstein, R. Judge, and S. Smadici, Demagnetizing fields in alloptical switching. 2017,

156. Wang, Y., J.Y. Park, Y.K. Koh, and D.G. Cahill, Thermoreflectance of metal transducers for time-domain thermoreflectance. J. Appl. Phys., 2010. 108: p. 043507,

157. Wilson, R.B., B.A. Apgar, L.W. Martin, and D.G. Cahill, Thermoreflectance of metal transducers for optical pump-probe studies of thermal properties. Opt. Express, 2012. 20(27): p. 28829,

158. Jewell, J.M., Thermooptic coefficients of soda-lime-silica glasses. J. Am. Ceram. Soc., 1993. 76(7): p. 1855,

159. Sudrie, L., M. Franco, B. Prade, and A. Mysyrowicz, Sudy of damage in fused silica induced by ultra-short IR laser pulses. Opt. Comm., 2001. 191: p. 333,

160. Schaffer, C.B., A. Brodeur, J.F. Garca, and E. Mazur, Micromachining bulk glass by use of femtosecond laser pulses with nanojoule energy. Opt. Lett., 2001. 26(2): p. 93,

161. Schaffer, C.B., J.F. Garca, and E. Mazur, Bulk heating of transparent materials using a high-repetition-rate femtosecond laser. Appl. Phys. A, 2003. 76: p. 351,

162. Hnatovsky, C., R.S. Taylor, E. Simova, V.R. Bhardwaj, D.M. Rayner, and P.B. Corkum, Polarization-selective etching in femtosecond laser-assisted microfluidic channel fabrication in fused silica. Opt. Lett., 2005. 30(14): p. 1867,

163. Cheng, G., K. Mishchik, C. Mauclair, E. Audouard, and R. Stoian, Ultrafast laser photoinscription of polarization sensitive devices in bulk silica glass. Opt. Express, 2009. 17(12): p. 9515, 
164. Ogawa, S., H. Nagano, and H. Petek, Hot-electron dynamics at Cu100, Cu110, and Cu111, surfaces: Comparison of experiment with Fermi-liquid theory. Phys. Rev. B, 1997. 55: p. 10869,

165. Ogawa, S., H. Nagano, and H. Petek, Optical intersubband transitions and femtosecond dynamics in Ag/Fe(100) quantum wells. Phys. Rev. Lett., 2002. 88: p. 116801,

166. Gall, D., Electron mean free path in elemental metals. J. Appl. Phys., 2016. 119: p. 085101,

167. Capinski, W.S., H.J. Maris, T. Ruf, M. Cardona, K. Ploog, and D.S. Katzer, Thermalconductivity measurements of GaAs/AlAs superlattices using a picosecond optical pump-and-probe technique. Phys. Rev. B, 1999. 59: p. 8105,

168. Ju, Y.S. and K.E. Goodson, Phonon scattering in silicon films with thickness of order 100 nm. Appl. Phys. Lett., 1999. 74: p. 3005,

169. Minnich, A.J., J.A. Johnson, A.J. Schmidt, K. Esfarjani, M.S. Dresselhaus, K.A. Nelson, and G. Chen, Thermal conductivity spectroscopy technique to measure phonon mean free paths. Phys. Rev. Lett., 2011. 107: p. 095901,

170. Johnson, J.A., A.A. Maznev, J. Cuffe, J.K. Eliason, A.J. Minnich, T. Kehoe, C.M. Sotomayor Torres, G. Chen, and K.A. Nelson, Direct measurement of roomtemperature nondiffusive thermal transport over micron distances in a silicon membrane. Phys. Rev. Lett., 2013. 110: p. 025901,

171. Hochbaum, A.I., R. Chen, R.D. Delgado, W. Liang, E.C. Garnett, M. Najarian, A. Majumdar, and P. Yang, Enhanced thermoelectric performance of rough silicon nanowires. Nature, 2008. 451: p. 163,

172. Chiritescu, C., D.G. Cahill, N. Nguyen, D. Johnson, A. Bodapati, P. Keblinski, and P. Zschack, Ultralow thermal conductivity in disordered, layered WSe2 crystals. Science, 2007. 315: p. 351,

173. Pernot, G., M. Stoffel, I. Savic, F. Pezzoli, P. Chen, G. Savelli, A. Jacquot, J. Schumann, U. Denker, I. Monch, C. Deneke, O.G. Schmidt, J.M. Rampnoux, S. Wang, M. Plissonnier, A. Rastelli, S. Dilhaire, and N. Mingo, Precise control of thermal conductivity at the nanoscale through individual phonon-scattering barriers. Nat. Mater., 2010. 9: p. 491,

174. Bae, M.H., Z. Li, Z. Aksamija, P.N. Martin, F. Xiong, Z.Y. Ong, I. Knezevic, and E. Pop, Ballistic to diffusive crossover of heat flow in graphene ribbons. Nat. Commun., 2013. 4: p. 1734,

175. Chen, G., Size and Interface Effects on Thermal Conductivity of Superlattices and Periodic Thin-Film Structures. J. Heat Transfer, 1997. 119: p. 220,

176. Asheghi, M., K. Kurabayashi, R. Kasnavi, and K.E. Goodson, Thermal conduction in doped single-crystal silicon films. J. Appl. Phys., 2002. 91: p. 5079, 
177. Liu, W. and M. Asheghi, Phonon-boundary scattering in ultrathin single-crystal silicon layers. Appl. Phys. Lett., 2004. 84: p. 3819,

178. Sergeev, A.V., Electronic Kapitza conductance due to inelastic electron-boundary scattering. Phys. Rev. B, 1998. 58: p. R10199,

179. Majumdar, A. and P. Reddy, Role of electron-phonon coupling in thermal conductance of metal-nonmetal interfaces. Appl. Phys. Lett., 2004. 84: p. 4768,

180. Mahan, G.D., Kapitza thermal resistance between a metal and a nonmetal. Phys. Rev. B, 2009. 79: p. 075408,

181. Lombard, J., F. Detcheverry, and S. Merabia, Influence of the electron-phonon interfacial conductance on the thermal transport at metal/dielectric interfaces. J. Phys.: Condens. Matter, 2015. 27: p. 015007,

182. Majumdar, A., Microscale heat conduction in dielectic thin films. J. Heat Transfer, 1993. 115: p. 7,

183. Chen, G., Thermal conductivity and ballistic-phonon transport in the cross-plane direction of superlattices. Phys. Rev. B, 1998. 57: p. 14958,

184. Siemens, M.E., Q. Li, R. Yang, K.A. Nelson, E.H. Anderson, M.M. Murnane, and H.C. Kapteyn, Quasi-ballistic thermal transport from nanoscale interfaces observed using ultrafast coherent soft X-ray beams. Nat. Mater., 2010. 9: p. 26,

185. Juve, V., M. Scardamaglia, P. Maioli, A. Crut, S. Merabia, L. Joly, N. Del Fatti, and F. Vallee, Cooling dynamics and thermal interface resistance of glass-embedded metal nanoparticles. Phys. Rev. B, 2009. 80: p. 195406,

186. Shen, S., A. Narayanaswamy, and G. Chen, Surface phonon polaritons mediated energy transfer between nanoscale gaps. Nano Lett., 2009. 9(8): p. 2909,

187. Nissim, Y.I., A. Lietoila, R.B. Gold, and J.F. Gibbons, Temperature distributions produced in semiconductors by a scanning elliptical or circular CW laser beam. J. Appl. Phys., 1980. 51(1): p. 274,

188. Burgener, M.L. and R.E. Reedy, Temperature distributions produced in a twolayer structure by a scanning CW laser or electron beam. J. Appl. Phys., 1982. 53(6): p. 4357,

189. Bauerle, D., Laser Processing and Chemistry. 2011.

190. Kryder, M.H., E.C. Gage, T.W. McDaniel, W.A. Challener, R.E. Rottmayer, G. Ju, Y.T. Hsia, and M.F. Erden, Heat assisted magnetic recording. Proc. IEEE, 2008. 96: p. 1810,

191. Weller, D. and A. Moser, Thermal effect limits in ultrahigh-density magnetic recording. IEEE Trans. Magn., 1999. 35: p. 4423,

192. Mimura, Y., N. Imamura, T. Kobayashi, A. Okada, and Y. Kushiro, Magnetic properties of amorphous alloy films of Fe with $\mathrm{Gd}, \mathrm{Tb}, \mathrm{Dy}, \mathrm{Ho}$, or Er. Journal of Applied Physics, 1978. 49(3): p. 1208, 10.1063/1.325008. 
193. Chang, Y.-J., A. Canizo-Cabrera, V. Garcia-Vazquez, Y.-H. Chang, and T.-h. Wu, Effect of Ta thickness on the perpendicular magnetic anisotropy in $\mathrm{MgO} / \mathrm{CoFeB} / \mathrm{Ta} /[\mathrm{Co} / \mathrm{Pd}] \mathrm{n}$ structures. Journal of Applied Physics, 2013. 114(18): $\mathrm{p}$. 184303, 10.1063/1.4829915.

194. Akhtar, M., Synthesis and fundamental property studies of energy material under high pressure., in Physics and Astronomy. 2017, University of Louisville, 10.18297/etd/2621.

195. Akhtar, M., G. Anderson, R. Zhao, A. Alruqi, J.E. Mroczkowska, G. Sumanasekera, and J.B. Jasinski, Recent advances in synthesis, properties, and applications of phosphorene. npj 2D Materials and Applications, 2017. 1(1): p. 5, 10.1038/s41699-017-0007-5.

196. Akhtar, M., M. Menon, M. Sunkara, G. Sumanasekera, A. Durygin, and J.B. Jasinski, High-pressure synthesis of rhombohedral $\alpha$-AgGaO2 via direct solid state reaction. Journal of Alloys and Compounds, 2015. 641: p. 87-92, 10.1016/j.jallcom.2015.04.051.

197. Akhtar, M., S. Pishgar, G. Sumanasekera, and J. Jasinski. In-situ Raman and PL spectroscopy of phosphorene under high-pressure. in APS March Meeting 2017. 2017. New Orleans, Louisiana (USA): Bulletin of the American Physical Society, http://meetings.aps.org/Meeting/MAR17/Event/294809 http://meetings.aps.org/Meeting/MAR17/Session/R33.2

198. Zhao, R., R. Jayasingha, A. Sherehiy, R. Dharmasena, M. Akhtar, J.B. Jasinski, S.-Y. $\mathrm{Wu}, \mathrm{V}$. Henner, and G.U. Sumanasekera, In Situ Transport Measurements and Band Gap Formation of Fluorinated Graphene. The Journal of Physical Chemistry C, 2015. 119(34): p. 20150-20155, 10.1021/acs.jpcc.5b06111.

199. Ziolkowska, D.A., J.S.D. Jangam, G. Rudakov, T.M. Paronyan, M. Akhtar, G.U. Sumanasekera, and J.B. Jasinski, Simple synthesis of highly uniform bilayercarbon nanocages. Carbon, 2017. 115: p. 617-624, 10.1016/j.carbon.2017.01.055.

200. Bäuerle, D., Instabilities and Structure Formation, in Laser Processing and Chemistry, D. Bäuerle, Editor. 2011, Springer Berlin Heidelberg: Berlin, Heidelberg. p. 623-677. 


\section{CURRICULUM VITA}

NAME: $\quad$ Farzaneh Hoveyda Marashi

ADDRESS: Department of Physics and Astronomy

102 Natural Science Building

University of Louisville

Louisville, KY 40292

\section{EDUCATION:}

BSc in Physics (Atomic, molecular, and optical physics)

Shahid Beheshti University, Iran

$2001-2007$

MSc in Physics

University of Mysore, India

2007 - 2010

MSc and Ph.D. in Physics (Experimental Condensed Matter)

2010 - 2017

University of Louisville, USA

AWARD \& FUNDING:

Graduate Network in Arts and Sciences (GNAS) Research Fund, 2017

MMM Conference Travel Grant, 2017

Arts \& Sciences Research and Creative Activities Grant, 2017

Doctoral Dissertation Completion Award, University of Louisville, 2017

John Dillon Jr Physics Fellowship (2015-2016)

Schwartz Award, outstanding graduate student, 2012

PROFESSIONAL SOCIETIES:

IEEE magnetics Society (2016-present)

American Physical Society (2012-present)

Society of Photo-Optical Instrumentation Engineers (SPIE) (2013-present) 